\title{
ENVIRONMENTAL VARIATION AND ITS EFFECT ON THE SUCCESS OF CROP- WILD HYBRIDIZATION IN THE RAPHANUS SPECIES COMPLEX
}

\author{
by \\ Kruti Shukla \\ M.Sc, University of Guelph, 2013 \\ B.Sc, University of Guelph, 2009 \\ A dissertation \\ presented to Ryerson University \\ in partial fulfillment of the \\ requirements for the degree of \\ Doctor of Philosophy \\ in the program of \\ Molecular Science
}

Toronto, Ontario, Canada, 2019

(C) Kruti Shukla, 2019 


\section{AUTHOR'S DECLARATION FOR ELECTRONIC SUBMISSION OF A DISSERTATION}

I hereby declare that I am the sole author of this dissertation. This is a true copy of the dissertation, including any required final revisions, as accepted by my examiners.

I authorize Ryerson University to lend this dissertation to other institutions or individuals for the purpose of scholarly research.

I further authorize Ryerson University to reproduce this dissertation by photocopying or by other means, in total or in part, at the request of other institutions or individuals for the purpose of scholarly research.

I understand that my dissertation may be made electronically available to the public. 


\section{Abstract \\ ENVIRONMENTAL VARIATION AND ITS EFFECT ON THE SUCCESS OF CROP- WILD HYBRIDIZATION IN THE RAPHANUS SPECIES COMPLEX}

Kruti Shukla, Doctor of Philosophy, 2019

Molecular Science, Ryerson University

Hybridization has been suggested as mechanism that can contribute to adaptive evolution and the success of crop-wild hybrid populations; but this response appears to depend upon environmental context. I explore how environmental variation affects crop trait expression, the strength and direction of selection on crop traits in radish weed populations, and the influence environmental variation has on crop-trait introgression across agricultural landscapes. Using the Raphanus crop-wild complex as a model system to study the environmental sensitivity of crop gene flow into weed populations, I first planted advanced-generation wild and crop-wild hybrid radish plants (that had previously evolved for three generations under relatively dry, relatively wet, or ambient control soil moisture or water-evolved conditions) into sheltered common gardens that were watered with low, ambient, or high soil moisture. From this work, hybridization and watering history did not enhance the success of advanced-generation hybrid plants relative to wild progenitors in Ontario, Canada. Next, I explored how phenotypic plasticity in response to environmental variation may distort a commonly used metric to measure the rate of evolution, the haldane. To determine the extent that plasticity affected estimates of evolutionary rate, I compared haldane estimates of advanced-generation water-evolved plants grown in a common garden that did not involve manipulation of ambient watering conditions. Estimates of the magnitude and direction of contemporary evolution differed significantly due to annual environmental variation, particularly for wild populations. Thus, I propose changes to the 
use of these equations and changes to the equation itself to help avoid generating false estimates of evolutionary rates. Finally, a meta-analysis of radish phenology and fecundity data collected from the last twelve years across four locations revealed that geography can affect the strength and direction of selection on crop- derived traits in weedy radish populations. This large, integrated study offers environmental risk assessment a new perspective on the role of environmental change on the success of crop-wild hybridization and its ability to generate weedy species. In summary, I provide evidence that environmental variation should be considered before making predictions about a crop trait's evolutionary trajectory and persistence in a weedy plant population. 


\section{Acknowledgements}

First, I would like to thank my advisor Lesley G. Campbell for all her unwavering support during this crazy ride. Her enthusiasm, guidance, and mentorship has been a motivating force during this $\mathrm{PhD}$. Through our day-to-day interactions over the past four years we have both evolved (ha!) and grown as people and I am appreciative of the opportunity you have given me thank you from the bottom of my heart. Thank you to my advisory committee members: Drs. Hafiz Maherali, Michael Arts, and Stephanie Melles and co-author Andrew Laursen for your advice, guidance, and sincerity.

Thank you to my parents (Piyush and Toral Shukla) and my brother and his family for emotionally supporting me on this whirlwind of an adventure. I appreciate the encouragement and constant reinforcement you provided every single day and making sure I was taken care of. To Sudir, you have been an unconditional, loving support. Thank you for that you do and all that you have done. You keep me fed and happy and I love you. More importantly, you make a wonderful research assistant. Thank you for all your help with experimental set-up, data collection, data entry, emotional breakdowns, everything - you're an honorary scientist in my book!

I am grateful to all the amazing friends that have kept me going. First and foremost, Tarn Preet Parmar - we met as co-lab mates and grew to become amazing friends. We've gone through this wild journey together and I appreciate all your help. Our late nights at the lab collecting/analyzing data, our research talks, and technical support (obviously!) have gotten me through some intense times and I could not have done this without you. To my friends Lisa Huynh, Priscilla Sreedharan, and Nancy Lay thank you for your continuous love and dedication. I appreciate the rant sessions to the beer, dinners, and more beer - love you all. To Laruen Des Marteaux and Aurora Patchett even though you're far away, it never felt that way. You were always there when I needed advice or a voice of reason. You have helped me through some difficult moments and am grateful to have you both in my life. Finally, thank you to all the amazing students that have helped me during my four years. 


\section{Contribution of Authors and Chapter Acknowledgements}

\section{Chapter 2}

Contributing Authors: K. Shukla (KS), Lesley G. Campbell (LGC), Andrew E. Laursen (AEL), Hafiz Maherali (HM), Jessica Benevides (JB), Neda Ejbari (NE).

I appreciate the many students, too numerous to name, assisted in population maintenance and data collection between 2010-2016. The staff of the University of Toronto's Koffler Scientific Research provided logistical support. The authors gratefully acknowledge the funding support from the Natural Sciences and Engineering Research Council of Canada (NSERC) Discovery Grants program (no. 402305-2011 to LGC), Ontario Graduate Scholarship (OGS), Queen Elizabeth II Graduate Scholarship (QEII), and the Faculty of Science Ryerson University (for a research fellowship to KS).

KS wrote the manuscript and ran the data analysis with support from LGC, HM, and AEL. KS, JB, and NE helped with experimental setup, data collection, and data analysis.

\section{Chapter 3}

Contributing Authors: K. Shukla (KS), Lesley G. Campbell (LGC), Hafiz Maherali (HM), Andrew E. Laursen (AEL), Jessica Benevides (JB), and Serena Sbrizzi (SS).

We particularly appreciate insights of A. Snow, K. Mercer, and A. Weis in the development of this study and thankful to M.T. Arts and S. Melles for helpful edits to the MS. Many students, too numerous to name, assisted in population maintenance and data collection between 2010-2016. The staff of the University of Toronto's Koffler Scientific Research provided logistical support. The authors gratefully acknowledge the funding support from the Natural Sciences and Engineering Research Council of Canada (NSERC) Discovery Grants program (no. 402305-2011 to LGC), Ontario Graduate Scholarship (OGS), Queen Elizabeth II Graduate Scholarship (QEII), and the Faculty of Science Ryerson University (for a research fellowship to KS).

$\mathrm{KS}$ wrote the manuscript and ran the data analysis with support from LGC, HM, and AEL. KS, JB, and SS helped with experimental setup, data collection, and data analysis. 


\section{Chapter 4}

Contributing Authors: K. Shukla (KS), Lesley G. Campbell (LGC), Steve Hovick (SH), Shion Song (SS), Zachary Teitel (ZT).

We particularly appreciate insights of A. Snow, K. Whitney, and R. Baucom in the development of this study. Many student researchers, too numerous to name, assisted in data collection in MI, ON, OH, and TX. The staff of the University of Michigan Biological Station, Koffler Scientific Reserve in Ontario, Canada, and Ohio State University Waterman Farm. Koffler Field Station and Ecological Reserves provided incredible field support. Funding was provided by NSERC Discovery (no. 402305-2011 to LGC).

KS wrote the manuscript and ran the data analysis with support from LGC and SH. KS, LGC, SH, SS, and ZT helped with data collection and analysis. 


\section{Table of Contents}

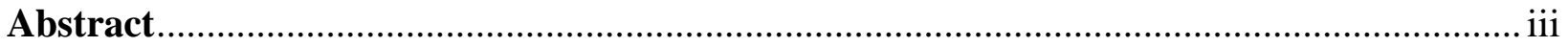

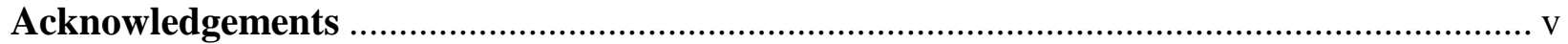

Contribution of Authors and Chapter Acknowledgements ................................................ vi

Table of Contents ........................................................................................................ vii

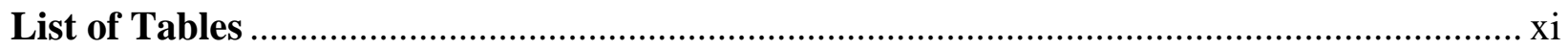

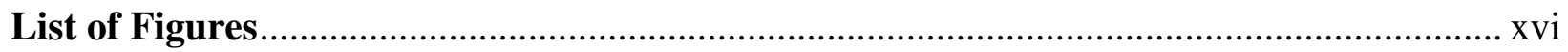

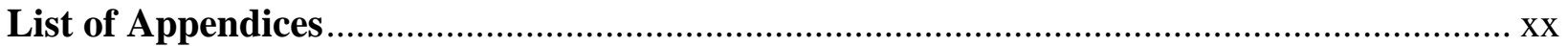

Chapter 1: Hybridization and its Sensitivity to Environmental Context ........................... 1

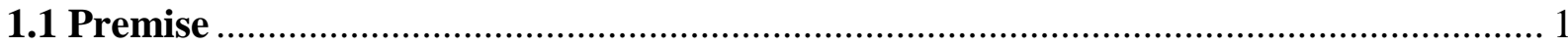

1.2 Hybridization as a Mechanism to Facilitate Adaptive Evolution - Crop-to-wild Gene

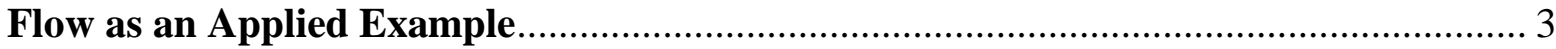

1.3 Rates of Evolution as a Metric for Measuring Change.......................................... 6

1.4 Geographic Variation in the Success of Hybrid Plants ......................................... 7

1.5 Experimental Environmental Clines as an Evolutionary Tool ................................ 8

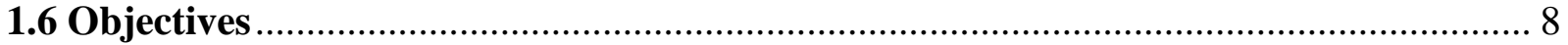

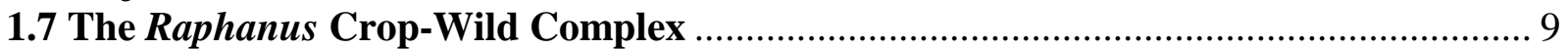

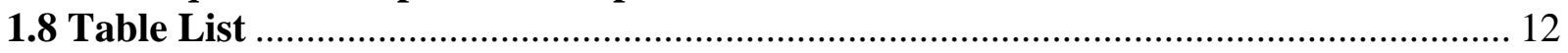

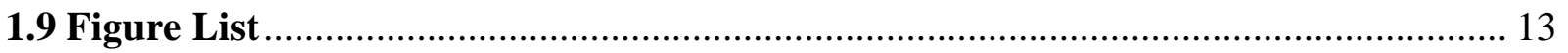

Chapter 2: Evolution of Advanced-Generation Hybrids After Climate Change and Crop Gene Introgression Does Not Increase Weediness of Crop-Wild Hybrids.................... 14

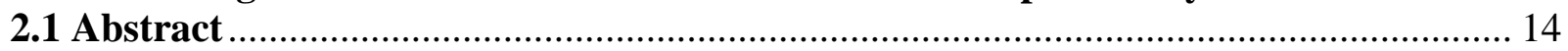

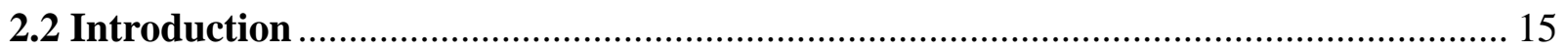

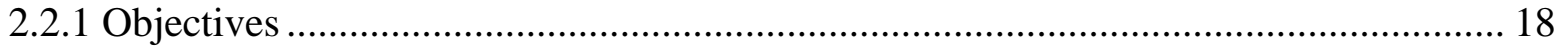

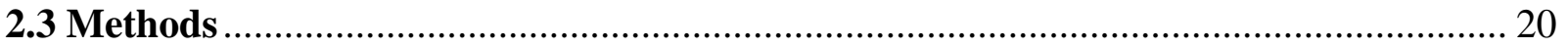

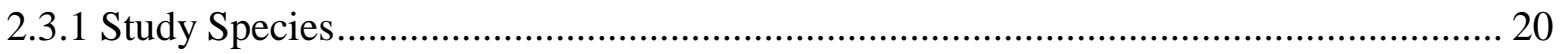

2.3.2 Study Site and Seed Sources ....................................................................... 20

2.3.3 Establishment of Replicated Populations under Watering Treatments and Common

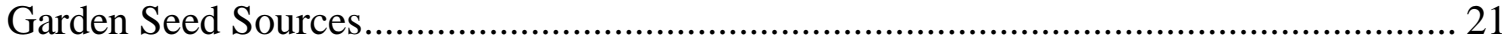

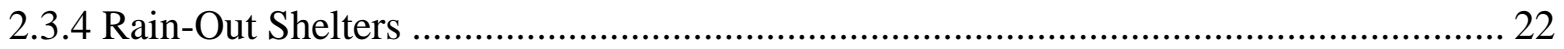

2.3.5 Common Garden Setup in 2015 and 2016....................................................... 23

2.3.6 Phenology and Life History Measurements ............................................................ 24

2.3.7 Crop-Specific Trait Data Collection .............................................................. 25

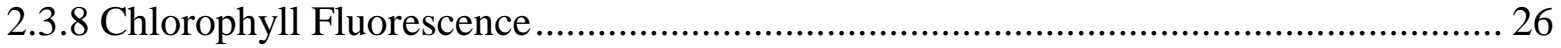

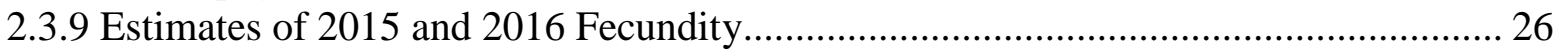

2.3.10 Statistical Analysis ............................................................................................. 27

2.3.10.1 Does the Frequency of Crop-Derived Alleles Vary Among Soil Moisture

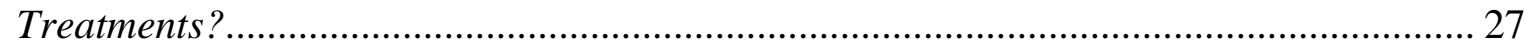

2.3.10.2 Allometric Experiment- Can Biomass Be Used as a Tool to Assess Fitness? ..... 28

2.3.10.3 Do Novel Environments Affect the Relative Success of Plants? ........................ 28

2.3.10.4 Does Watering History Predispose Plants to Greater Success? ........................ 28

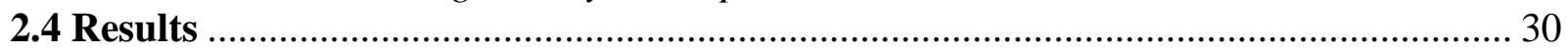

2.4.1 Evolution of Flower Colour in Hybrid Populations .............................................. 30

2.4.2 Can Biomass Be Used as a Correlate of Seed Set? ............................................... 30 
2.4.3 Do Novel Environments Affect the Life-History and Relative Fecundity of Hybrid Plants?

2.4.4 Does Watering History Predispose Hybrid Plants to Increased Fecundity Relative to

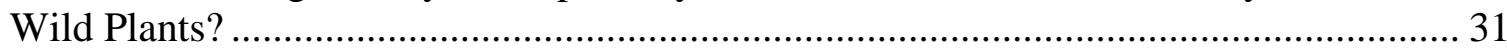

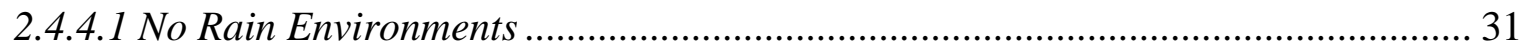

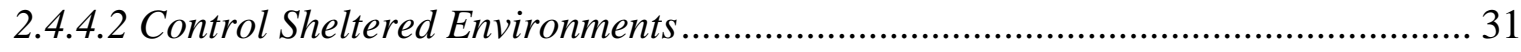

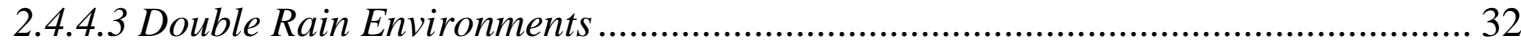

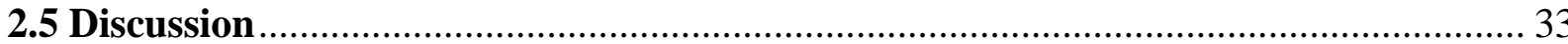

2.5.1 Persistence of Crop Traits in $\mathrm{G}_{5}$ Hybrids....................................................... 34

2.5.2 Allometric Models: Relative Importance of Hybridization and Selection on the

Success of Invasiveness in Raphanus Species ........................................................ 35

2.5.3 Environmental Context: An Important Determinant of Invasive Success in Radish

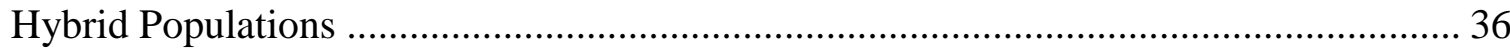

2.5.4 Environmental Selection History Can Affect Plant Survival and Fecundity .............. 37

2.5.5 The Influence of Climate on Hybridization and Mating Systems ............................. 39

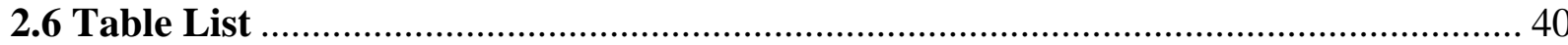

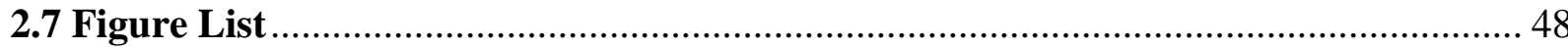

Chapter 3: Rate of Evolution Metrics are Sensitive to Environmental Context................. 56

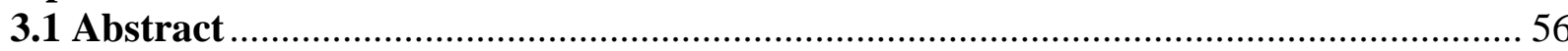

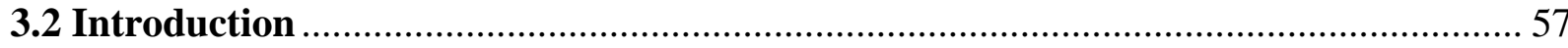

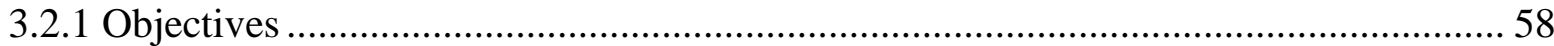

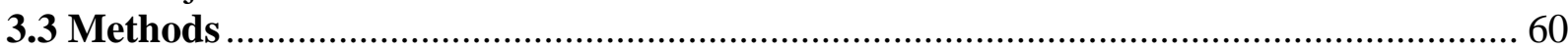

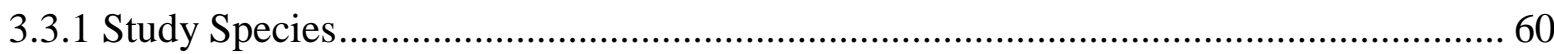

3.3.2 Seed History of Wild and Hybrid Radish Populations Used in my Experiment .......... 60

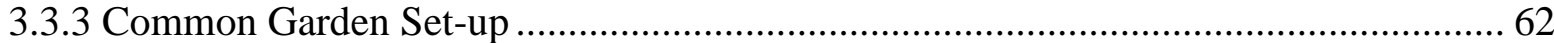

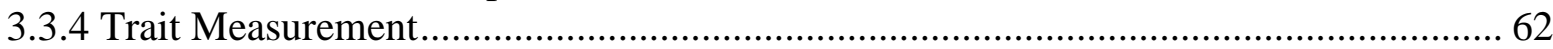

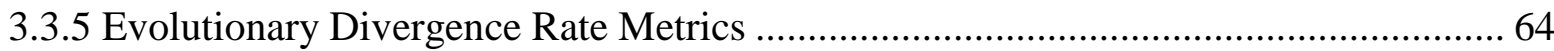

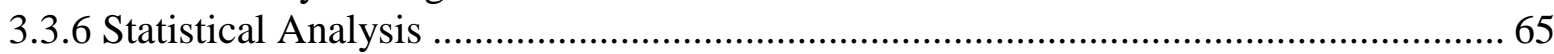

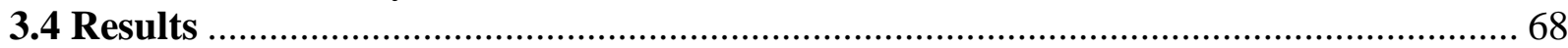

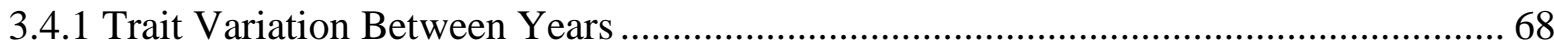

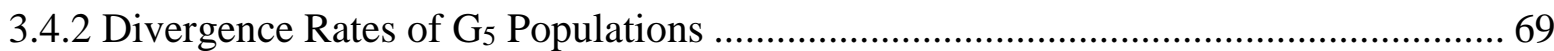

3.4.3 Correlations Between Divergence Rates When Reference and Selection Populations are Grown in Different Environments versus Common Gardens .................................. 69

3.4.4 Consistency Between Environments of Divergence Rates of Populations Grown in

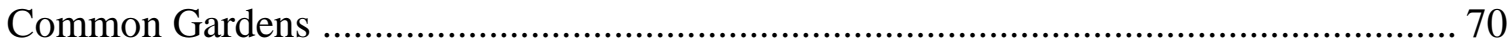

3.4.5 Correlations among Divergence Rate Estimates Between Years .............................. 71

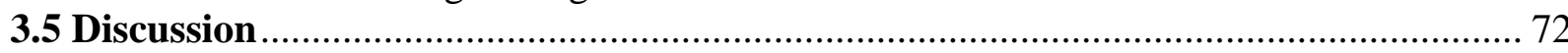

3.5.1 The Puzzle of Inconsistent Estimates of Evolutionary Rates ................................ 72

3.5.2 Selecting Traits for Simple Estimates of Rate of Evolution................................... 73

3.5.3 Phenotypic Plasticity Can Alter the Apparent Rate of Evolution ............................. 73

3.5.4 The Complexity of Measuring Rates of Evolution in Quantitative Traits: Moving

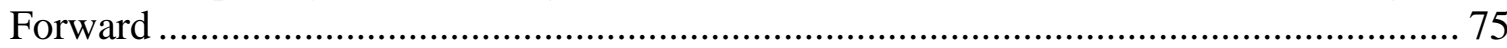

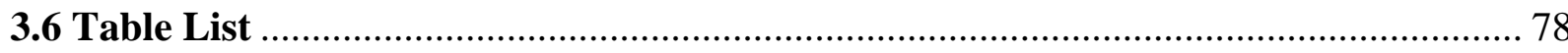

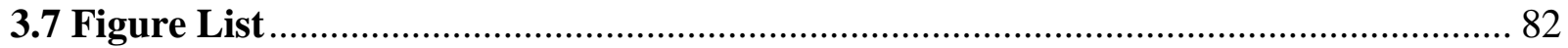


Chapter 4: Variation in Fitness and Selection on Crop traits in Crop-Wild Hybrid

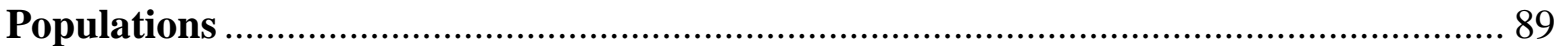

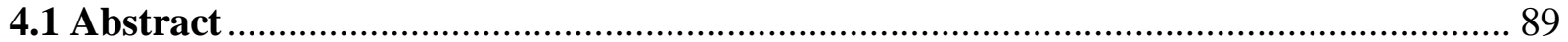

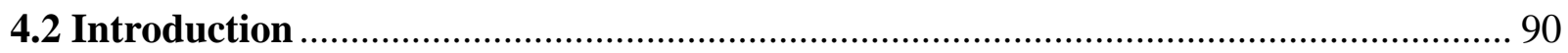

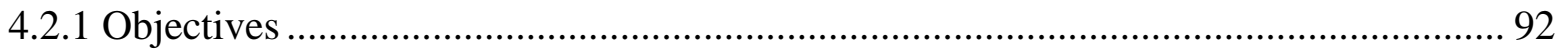

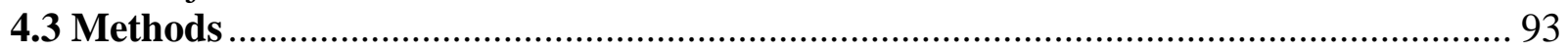

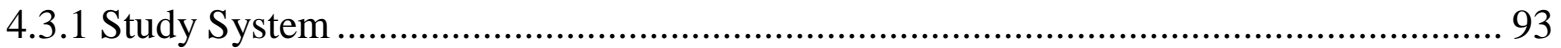

4.3.2 Seed Sources for Experimental Populations......................................................... 93

4.3.2.1 Michigan and Texas Plants......................................................................... 93

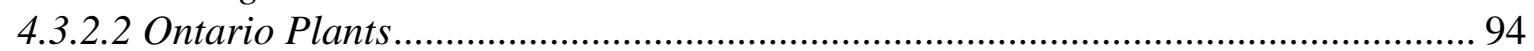

4.3.3 Data Sets ........................................................................................................... 95

4.3.4 General Method of Measuring Date of Emergence, Flowering, and Seed Set............ 95

4.3.5 Statistical Analysis ....................................................................................... 96

4.3.5.1 Life-History Trait Response ........................................................................... 96

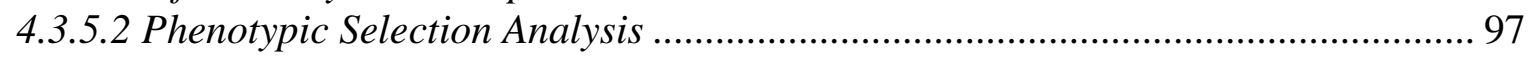

4.3.5.3 Interpretation of Selection Metrics ................................................................ 97

4.3.5.4 Differences in Linear, Quadratic, and Selection Differentials Across Locations . 98

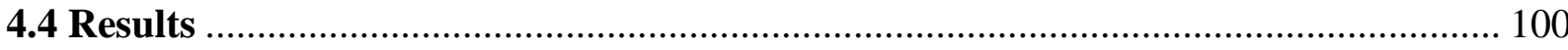

4.4.1 Trait Variation in Flower Colour, Emergence and Flowering Time, and Seed Set ... 100

4.4.2 Crop Trait Persistence ................................................................................. 101

4.4.3 Variation in the Strength of Selection After Hybridization.................................... 102

4.4.4 Variation in the Strength of Selection using Weighted Linear and Quadratic Models in

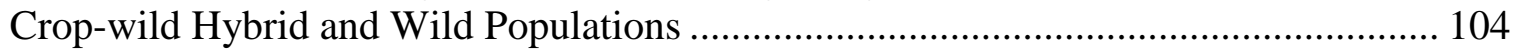

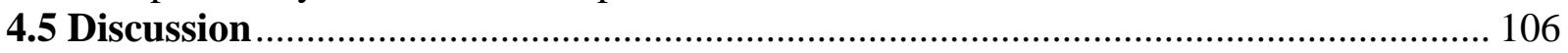

4.5.1 Persistence of Crop Traits in Hybrid Populations .............................................. 106

4.5.2 Seed Set and Variable Selection Strengths Across Hybrid Populations ................... 108

4.5.3 Moving Forward with Risk Assessment of Crop Gene Flow ................................. 109

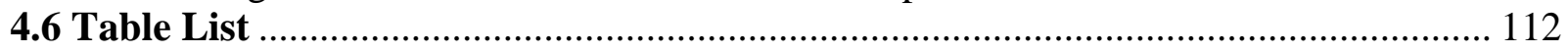

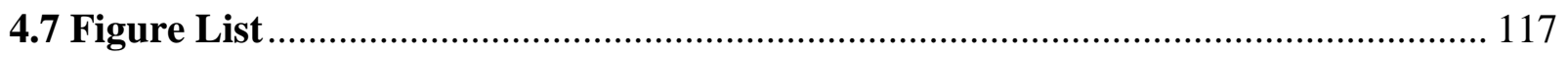

Chapter 5: Synthesis and the Future Study of Crop-Wild Hybridization ....................... 121

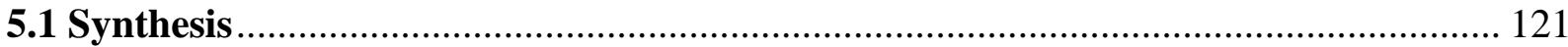

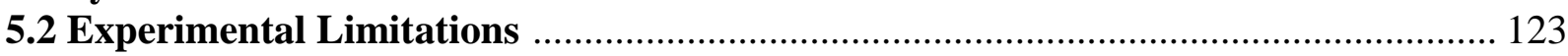

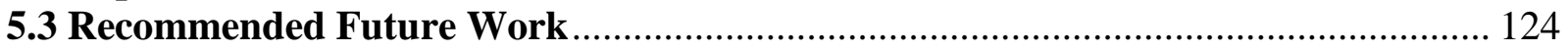

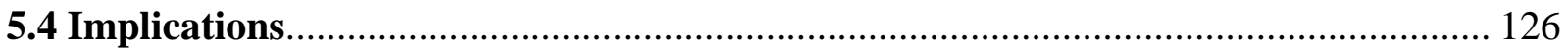

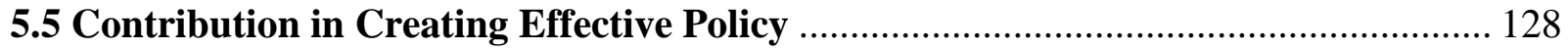

Appendix 1 - Frequency and Volume of Water Applied to Experimental Plots .............. 130

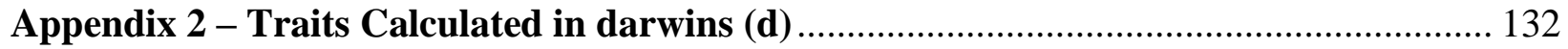

Appendix 3 - Additional Life History Traits Measured ............................................. 135

Appendix 4 - Reaction Norm Repeated-Measures Analysis........................................... 142

Appendix 5 - Divergence Rate Correlations Between Years for Genotype, Historical

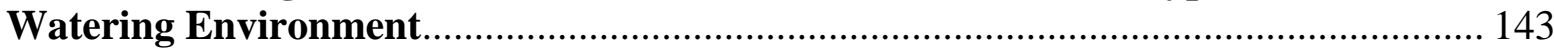

Appendix 6 - Source Population Data for Selection Analysis Study .............................. 145

Appendix 7 - Error Estimates on Selection Gradient Values in Michigan Populations.... 153

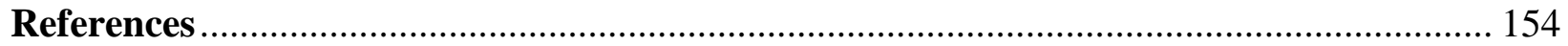




\section{List of Tables}

Table 1.1: Minimum, average, and maximum temperature and cumulative data in studies Campbell et al. 2006 (Michigan and California), Hovick et al. 2012 (Texas), Teitel et al. (2016) and this study (Ontario). The city and weather station code are presented in parenthesis after each location. The data presented are calculated from the growing season of each study: Michigan (May - September), California (January - June), Texas (February - July), and Ontario (May September). Data for studies in the USA (Michigan, California, and Texas) were collected from the Weather Underground website (www.wunderground.com) while Ontario data was collected from the Government of Canada website (www.climate.weather.gc.ca)...

Table 2.1: To determine if biomass could be used a proxy to estimate fitness, I ran a model testing the allometric relationship between biomass and the a) number of flowers, b) number of fruits, and c) number of seeds produced. Response variables were tested in response to Genotype (G: wild - W and hybrid - H), historical watering treatment (WH: no rain - NR, control unsheltered - CU, control sheltered - CS, double rain - DR), and the covariate of biomass (Bi), and their interactions therein. Correlation values $\left(\mathrm{r}^{2}\right)$ are presented for models where biomass significantly correlated with a factor (genotype and watering history). Analyses were run in RStudio (version 1.0.143). F-statistics are presented to indicate significant differences: $\mathrm{ns}, \mathrm{P}>$ $0.10 ;+, \mathrm{P}<0.10 ; *, \mathrm{P}<0.05 ; * *, \mathrm{P}<0.01 ; * * *, \mathrm{P}<0.001$.

Table 2.2: To estimate whether hybrid plants that invade novel environments perform better relative to wild plants, I ran a split-plot mixed model ANOVA on (a - d) several life history traits and (e) a single lifetime fecundity trait in response fixed effects current water treatment (CT: no rain-NR, control shelter-CS, double rain- DR), and genotype (G: wild, hybrid) as the split-plot factor, and their interactions, with shelter (S: 10 levels) as a random factor. Analyses were done on data collected from CS-evolved wild and hybrid plants that grew in NR, CS, and DR soil moisture environments. Across models, CT was tested against the whole plot error interaction [i.e., between subject's error; presented as $\operatorname{Error}(S \times C T)$ in the table] while the within subject's effects ( $\mathrm{G}$ and $\mathrm{G} \times \mathrm{CT}$ ) are tested against the residuals error terms (presented as Error in the table). Additionally, considering the non-orthogonality of the data, type III ANOVA results are presented in the table using a Kenward-Roger's adjustment for computing the degrees of freedom. Split-plot analyses were run in R-Studio (version 1.0.143) and SAS Enterprise Guide 61 (PROC GLM). Box-Cox transformations $(\lambda)$ and sample sizes (n) are reported for each response variable. F-statistics in the fixed effect ANOVA table are presented to indicate significant differences: $\mathrm{ns}, \mathrm{P}>0.10 ;+, \mathrm{P}<0.10 ; *, \mathrm{P}<0.05 ; * *, \mathrm{P}<0.01$; ***, $\mathrm{P}<0.001$

Table 2.3: To estimate whether watering history pre-disposes hybrid plants to perform better than wild plants, I ran a mixed model ANOVA on (a - d) several life history traits and (e) a single lifetime fecundity trait for each current watering environment (no rain- NR, control shelter-CS, and double rain-DR). For each watering environment, I ran traits in response to watering history (WH: NR plots: NR and CS; CS plots: control unsheltered and CS; DR plots: DR and CS) and genotype (wild and hybrid) as the fixed factors and block (10 levels) as the random factor. Considering the non-orthogonality of the data, type III ANOVA results are presented in the table using a Kenward-Roger's adjustment for computing the degrees of freedom. The fixed effects models compute the F-statistic using the mean square error of each 
model (presented as Error in the table). Traits that were analysed included a) days to seedling emergence, b) days to flower, c) leaf length, d) stem diameter, and e) above-ground biomass in each current watering environment. Additionally, each trait also lists the $\chi^{2}$ significance, and associated degrees of freedom, comparing the model with and without the block factor (i.e, measuring the significance of block in the model). Analyses were run in R-Studio (version 1.0.143) and SAS Enterprise Guide 61 where all response variables were fitted to a normal distribution and an identity link function. Box-Cox transformations $(\lambda)$ and sample sizes (n) are reported for each response variable. F-statistics in the fixed effect ANOVA table are presented to indicate significant differences: $\mathrm{ns}, \mathrm{P}>0.10 ;+, \mathrm{P}<0.10 ; *, \mathrm{P}<0.05 ; * *, \mathrm{P}<0.01 ; * * *, \mathrm{P}<$

0.001

Table 2.4: To evaluate whether water stress influenced photosynthesis, I compared the darkadapted quantum efficiency of photosystem II (PSII) across wild and hybrid CS plants grown in all current watering treatments. (a) To estimate whether hybrid plants (with a shared control sheltered watering history) that invaded novel environments (current watering treatments [CT]: no rain-NR, control shelter-CS, double rain- DR) have higher quantum efficiency relative to wild plants, I ran a split-plot mixed model ANOVA on chlorophyll fluorescence in response to current water treatment (CT:), and genotype (wild, hybrid) as the split factor, and interactions therein as fixed factors and block as a random factor. Next, to estimate whether watering history predisposes hybrid plants to have higher PSII efficiency than wild plants, I ran a mixed model ANOVA on plants in current watering treatments of (b) no rain plots, (c) control sheltered plots, and (d) double rain plots, respectively, on chlorophyll fluorescence values in response to genotype (wild and hybrid) and watering history (in current NR plots: NR and CS WH; current CS plots: control unsheltered and CS WH; and current DR plots: DR and CS WH), and their interactions as the fixed factors and block as the random factor. Considering the nonorthogonality of the data, type III ANOVA results are presented in the table using a KenwardRoger's adjustment for computing the degrees of freedom. The fixed effects models compute the F-statistic using the mean square error of each model. Additionally, each model also lists the $\chi^{2}$ significance and associated degrees of freedom for the effect of block (i.e., for each trait in each watering environment). Analyses were run in R-Studio (version 1.0.143) and SAS Enterprise Guide 61 where all response variables were fitted to a normal distribution and an identity link function. Box-Cox transformations $(\lambda)$ and sample sizes (n) are reported for each response variable. F-statistics in the fixed effect ANOVA table are presented to indicate significant differences: $\mathrm{ns}, \mathrm{P}>0.10 ;+, \mathrm{P}<0.10 ; *, \mathrm{P}<0.05 ; * *, \mathrm{P}<0.01 ; * * *, \mathrm{P}<$ 0.001

Table 3.1 Mixed model ANOVA of mean trait plasticity of) days to emergence and b) days to flowering between years in response to genotype (wild versus hybrid), watering history (WH: no rain, control sheltered, control unsheltered, and double rain), and their interaction as fixed factors, with Block (20 levels) as a random factor. Considering the non-orthogonality of the data, type III ANOVA results are presented in the table using a Kenward-Roger's adjustment for computing the degrees of freedom. The fixed effects models compute the F-statistic using the mean square error of each model (presented as Error in the table). Additionally, for each trait a $\chi^{2}$ significance test was run comparing the model with and without the block factor (i.e, measuring the significance of block in the model). (a) The model measuring days to emergence had a significant block effect $\left[\chi_{(\mathrm{df}=1)}^{2}=43.50, \mathrm{p}<0.001\right]$. (b) The model measuring days to 
flowering had a significant block effect $\left[\chi_{(\mathrm{df}=1)}^{2}=27.54, \mathrm{p}<0.001\right]$. Analyses were run in R-Studio (version 1.0.143) and SAS Enterprise Guide 61 where all response variables fitted to a normal distribution and an identity link function. F-statistics in the fixed effect ANOVA table are presented to indicate significant differences: $\mathrm{ns}, \mathrm{P}>0.10 ;+, \mathrm{P}<0.10 ;{ }^{*}, \mathrm{P}<0.05 ; * *, \mathrm{P}<0.01$;

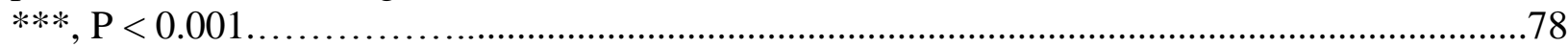

Table 3.2: Mixed model ANOVA of phenotypic differences for a) frequency of white-flowered plants, b) pollen fertility, and c) chlorophyll fluorescence between years. Considering the nonorthogonality of the data, type III ANOVA results are presented in the table using a KenwardRoger's adjustment for computing the degrees of freedom. The fixed effects models compute the F-statistic using the mean square error of each model (presented as Error in the table).

Additionally, a $\chi^{2}$ significance test was run comparing the model with and without the block factor (i.e, measuring the significance of block in the model). (a) White colour frequency was tested in response to watering history (WH: no rain, control sheltered, control unsheltered, and double rain), year $(2015,2016)$, and their interaction as fixed factors, with block (levels $=20)$ as a random factor. The model did not have a significant block effect [ $\left.\chi^{2}(\mathrm{df}=1)=9.09 \times 10^{-13}, \mathrm{p}=1\right]$. (b) Pollen fertility was tested in response to genotype (G: wild, hybrid), watering history, year, and their interactions as fixed factors, with block (levels $=20$ ) as a random factor. The model had a significant block effect $\left[\chi^{2}(\mathrm{df}=1)=56.61, \mathrm{p}<0.001\right]$. (c) Chlorophyll fluorescence was tested in response to genotype, watering history, year, and their interactions as fixed factors, with block $($ levels $=20)$ as a random factor. The model did not have a significant block effect $\left[\chi_{(\mathrm{df}=1)}^{2}=4.55 \times 10^{-13}, \mathrm{p}=1\right]$. Analyses were run in R-Studio (version 1.0.143) and SAS Enterprise Guide 61 where all response variables were fitted to a normal distribution and an identity link function. F-statistics in the fixed effect ANOVA table are presented to indicate significant differences: $\mathrm{ns}, \mathrm{P}>0.10 ;+, \mathrm{P}<0.10$;, $\mathrm{P}<0.05$; **, $\mathrm{P}<0.01$; ***, $\mathrm{P}<$ 0.001

Table 3.3: Repeated measures fixed-factor ANOVA of divergence rates of three traits [ a) days to emergence, b) days to flowering, c) frequency of white-flowered plants] and their response to genotype (wild versus hybrid), watering history (WH: no rain, control unsheltered, and double rain), and their interaction, with Year as the repeated measure. In models measuring (a) emergence time and (b) flowering time, 27 populations, in total, were considered when testing the between subject's effects. (c) The white flower colour frequency model considered 14 populations when testing the between subject's effects. The error term used for within subject's and between subject's effects are listed in the ANOVA tables under each respective category along with each model's sample size (n). F-statistics are presented to indicate significant differences: $\mathrm{ns}, \mathrm{P}>0.10 ;+, \mathrm{P}<0.10 ; *, \mathrm{P}<0.05 ; * *, \mathrm{P}<0.01 ; * * *, \mathrm{P}<$ 0.001

Table 3.4: Correlation coefficients $(r)$ that measure the relationship between divergence rates estimated using evolved and reference populations from the same common garden (a correct estimation method) versus divergence rates estimated using evolved plants and reference populations grown in different environments (an incorrect estimation method). The common garden year refers to which evolved plant populations were used in the estimation of divergence rates. P-values estimated for each correlation coefficient are also presented. 
Table 4.1: ANOVA model testing the white flower colour frequency across years in a) Michigan and b) Ontario populations. a) In Michigan an ANCOVA model was run testing changes white flower colour frequency across two years $(2004,2005)$ and four generations (Generations: 1, 3, $5,10)$, with generation acting as a covariate. b) In Ontario an ANOVA model was run testing changes in white flower colour frequency across four years $(2013,2014,2015,2016)$. Generation was inherently correlated with year between 2013 and 2014 but 2015 and 2015 populations represented a single generation (generation 5). Due to this confounding variable, generation could not be run as a covariate in the Ontario model. F-statistics are presented; to indicate significant differences: $\mathrm{ns}, \mathrm{P}>0.10 ;+\mathrm{P}<0.10 ; *, \mathrm{P}<0.05 ; * *, \mathrm{P}<0.01 ; * * *, \mathrm{P}<$ 0.001

Table 4.2: ANCOVA model mean trait responses of days to emergence, days to flower, and number of seeds per plant (seed set) in a) Michigan wild and hybrid populations, b) Ontario wild and hybrid populations, c) Michigan hybrid populations, and d) Ontario hybrid populations. Numerator and denominator degrees of freedom are represented in subscripts for each parameter. Analyses were run in R-Studio Version 1.0.143. F-statistics are presented to indicate significant differences: $\mathrm{ns}, \mathrm{P}>0.10 ;+\mathrm{P}<0.10 ; *$, $\mathrm{P}<0.05$; **, $\mathrm{P}<0.01 ; * * *, \mathrm{P}<0.001 \ldots \ldots \ldots \ldots \ldots \ldots . . . . .113$

Table 4.3: The mean number of seeds per plant in Ontario hybrid populations across years. Mean \pm SE (groups marked with different letters represent significantly different groups).......114

Table 4.4: Wilcoxon signed-rank test comparing (a) directional selection gradients, (b) quadratic selection gradients, and (c) selection differentials between wild and crop-wild hybrid populations. Directional selection gradients (a) represent the direct linear relationship a trait has on the number of seeds per plant (i.e., fitness) whereas quadratic selection gradients (b) represent the non-linear relationship a trait has on fitness. Selection differentials (c) represent the total direct and indirect direction and quadratic selection, separately, occurring for the trait. In both (a) and (b) parameters, I used standard and weighted models for both traits. Standard models that evaluate the raw-value of the trait extracted from the phenotypic selection analysis (PSA). Weighted models used selection values weighted by variance component calculated in the standard PSA; populations with greater selection confidence (i.e., had less variance) are weighted more than populations with less selection confidence (i.e., greater variance). For a detailed description, see methods section 4.3.4.2. Analysis was run in IBM SPSS Statistics 24. Sample size $(\mathrm{N})$ and Wilcoxon signed-rank test statistics are presented; to indicate significant differences: $\mathrm{ns}, \mathrm{P}>0.10 ;+, \mathrm{P}<0.10 ; *, \mathrm{P}<0.05 ; * *, \mathrm{P}<0.01 ; * * *, \mathrm{P}<0.001$

Table 4.5: Directional $(\beta)$ and quadratic $(\gamma)$ selection gradients and selection differentials for seedling emergence and flowering times in a) Michigan wild and hybrid populations, b) Ontario wild and hybrid populations, c) Michigan hybrid populations, and d) Ontario hybrid populations. Numerator and denominator degrees of freedom are represented in subscripts for each parameter. Analyses were run in R-Studio Version 1.0.143. F-statistics are presented; to indicate significant differences: $\mathrm{ns}, \mathrm{P}>0.10 ;+, \mathrm{P}<0.10 ; *, \mathrm{P}<0.05 ; * *, \mathrm{P}<0.01 ; * * *, \mathrm{P}<0.001$

Table 4.6: Directional $(\beta)$ and quadratic $(\gamma)$ weighted selection gradients in a) Michigan wild and hybrid populations, b) Ontario wild and hybrid populations, c) Michigan hybrid populations, and 
d) Ontario hybrid populations. Numerator and denominator degrees of freedom are represented in subscripts for each parameter. Analyses were run in R-Studio Version 1.0.143. F-statistics are presented; to indicate significant differences: $\mathrm{ns}, \mathrm{P}>0.10 ;+, \mathrm{P}<0.10 ; *, \mathrm{P}<0.05 ; * *, \mathrm{P}<0.01$;

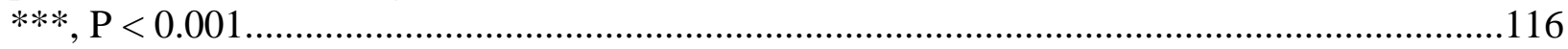




\section{List of Figures}

Figure 1.1: Interspecific gene flow, and successive hybrid progeny, can be driven by the environment plants are found in and the abiotic (e.g., temperature or moisture availability) and biotic (e.g., pollinators, con-specific competitors) factors they interact with. Hybridization rates, firstly, may be altered by the habitats progenitor species are found in, where changes in abiotic and biotic factors will affect the propensity for hybridization to occur. Once early-generation hybrid populations are created, the same abiotic and biotic hierarchy can facilitate establishment and the spread of advanced-generation hybrid populations.

Figure 2.1: Experimental area of 2015 (above) and 2016 (below) shelter common garden experiments at the Koffler Scientific Reserve. King City, Ontario. Thirty rain-out shelters (2.44 $\mathrm{m}$ by $3.05 \mathrm{~m}$ ) were constructed and erected in a randomized block design. In 2015, plots were established on a $20 \mathrm{~m} \times 18 \mathrm{~m}$ tilled plot of land and in 2016 plots were established on $25 \mathrm{~m} \times 25$ $\mathrm{m}$ tilled plots of land. The 2016 site was approximately 210 meters southeast of the 2015 site.

Figure 2.2: A photograph of rain-out shelters used to manipulate soil moisture in 2015 and 2016 common gardens. Shelters were $3.05 \mathrm{~m}$ by $2.44 \mathrm{~m}$ wooden frames with transparent sheet plastic stretched over the frame, acting as a roof that blocked rain but not to interfere with sunlight; new sheet plastic was applied each year. Using metal poles, frames were slanted and elevated to approximately $1.2 \mathrm{~m}$ above ground at their lowest corner. Frames were slanted to intercept and divert natural rainfall into a $208 \mathrm{~L}$ plastic collection barrel via an eavestrough attached to the lowest side of the wooden frame.

Figure 2.3: The watering history of source populations imposed from 2010 to 2014. Fifth generation seeds were planted in several combinations in one of three current treatments (Double Rain - DR, Control Shelter - CS, and No Rain - NR). Replicated common gardens of historic water evolved wild and hybrid plants and CS- wild and hybrid plants were reintroduced into each respective water treatment. For example, hybrid double rain $\left(\mathrm{H}_{\mathrm{DR}}\right)$ and wild double rain $\left(\mathrm{W}_{\mathrm{DR}}\right)$ evolved plants were planted with control-sheltered hybrid and wild plants $\left(\mathrm{H}_{\mathrm{CS}}\right.$ and $\mathrm{W}_{\mathrm{CS}}$, respectively) in a double rain common garden. Similarly, no-rain-evolved and control-shelteredevolved wild and hybrid plants were planted in a no-rain common garden.....

Figure 2.4: The frequency of white flower colour in hybrid populations from historic no-rain (pink-filled circle), control unsheltered (orange-filled square), control sheltered (teal-filled triangle), and double-rain (blue-filled inverted triangle) watering histories from $\mathrm{F}_{1}$ to $\mathrm{G}_{5}$. Dotted grey line represents the Hardy-Weinberg $(\mathrm{H}-\mathrm{W})$ expected frequency of white flower colour in the populations and black lines represent the mean white colour frequency ( \pm standard error) across all water selection histories

Figure 2.5: Comparing the average number of days wild or hybrid seedlings take to emerge from the soil when invading into novel and control current watering treatment environments. The graph displays the mean days to seedling emergence ( \pm standard error) of control shelter evolved wild and hybrid plants (wild - open circles, hybrid - filled circles) invading into novel environments (no rain and double rain environments) versus control sheltered environments. 
Figure 2.6: Comparing the average number of days wild and hybrid plants take to (a) emerge from the soil and (b) flower. Both graphs display the mean days ( \pm standard error) of wild and hybrid plants (wild - open circles, hybrid - filled circles) grown in no rain, control, and double rain environments. In each environment wild and hybrid plants represent individuals that have evolved in control sheltered environments and the current environment (no rain: control sheltered and no rain evolved wild and hybrid plants; control sheltered: control sheltered and control unsheltered evolved wild and hybrid plants; double rain: control sheltered and double rain evolved wild and hybrid plants)....

Figure 2.7: Comparing the average number of days control and water evolved plants take to (a) emerge from the soil and (b) flower. Both graphs display the mean days ( \pm standard error) of control (CS) and water-evolved (WH) plants (CS - open circles, WH - filled circles) grown in no rain, control, and double rain environments. In each environment plants represent individuals that have evolved in control sheltered environments and the current environment (no rain: control sheltered and no rain evolved plants; control sheltered: control sheltered and control unsheltered evolved plants; double rain: control sheltered and double rain evolved plants)

Figure 2.8: Comparing the quantum efficiency of the photosystem II (PS II) of plants that grew in double rain moisture environments. The graph displays average chlorophyll fluorescence ( \pm standard error) between wild and hybrid plant genotypes (wild - open circle, hybrid - closed circle) when grown in double rain environments. Wild and hybrid plants represent individuals that have evolved in double rain and control environments, collectively.

Figure 3.1: The watering history of wild and hybrid plant populations from 2010 to 2014. In 2010, crop and wild plants were planted in 36 plots as part of a randomized block design at the Waterman Farm at Ohio State University. The plots, from the parental generation $\left(\mathrm{G}_{0}\right)$, experienced one of four watering histories - double rain (DR), control sheltered (CS), control unsheltered (CU), and no rain (NR). Gene-flow naturally occurred between and among wild and cultivated-wild plants within the plots and gave rise to the first generation (i.e., $\mathrm{F}_{1}$ ) of wild and crop $\times$ wild hybrid $\left(R\right.$. sativus $\times R$. raphanistrum) seeds. Wild and hybrid $2^{\text {nd }}$ generation $\left(\mathrm{G}_{2}\right)$ to $4^{\text {th }}$ generation $\left(\mathrm{G}_{4}\right)$ plants ( 5 replicate populations per watering treatment) were grown under the same watering conditions at the Koffler Scientific Reserve (KSR) in King City, Ontario, Canada. Fifth generation plants were grown in 2015 and 2016 common gardens at KSR

Figure 3.2: Comparing the evolution of flowering phenology of $\mathrm{G}_{5}$ wild and hybrid populations grown under one of three environmental conditions from $\mathrm{G}_{2}-\mathrm{G}_{4}$ and then grown in a common garden in $\mathrm{G}_{5}$. Mean days to flower $( \pm \mathrm{SE}$ ) between (a) genotype by years (wild - open circles, hybrid - closed circles) and (b) watering history by genotype by years (NR-no rain, CU-control unsheltered, CS-control sheltered, DR-double rain; wild - open circles, hybrid - closed circles). Non-shared capital letters signify statistical differences between years. 
Figure 3.3: Comparing the evolution of mean pollen fertility of $\mathrm{G}_{5}$ wild and hybrid populations grown under one of three environmental conditions from $\mathrm{G}_{2}-\mathrm{G}_{4}$ and then grown in a common garden in $\mathrm{G}_{5}$. Graph displays mean percent pollen fertility $( \pm \mathrm{SE}$ ) between genotypes (wild open circles, hybrid - filled circles) between years. Letters above circles represent pair-wise differences, where non-shared letters signify statistical differences and shared letters represent no significant difference among treatments.

Figure 3.4: Comparing the evolution of mean quantum efficiency of PSII ( \pm SE) between G5 genotypes (wild - open circles, hybrid - filled circles) grown under one of three environmental conditions (watering histories: NR-no rain, CU-control unsheltered, CS-control sheltered, DRdouble rain) between common garden years. Non-shared capital letters signify statistical differences between years.

Figure 3.5: Comparing evolutionary rate estimates (presented in haldanes) of seedling emergence times $( \pm$ SE) across genotypes (wild - open circles, hybrid - closed circles) between years. Among wild populations, values represent the change between wild water-evolved plants and wild CS plants. Similarly, among hybrid populations, values represent the change between hybrid water-evolved and CS population.

Figure 3.6 Comparing evolutionary rate estimates of $\mathrm{G}_{5}$ wild and hybrid radish plants grown under one of three environmental conditions between common garden years. Divergence rates are presented in haldanes of (a) the frequency of white-flowered plants ( \pm SE) and (b) days to emergence $( \pm S E)$ across watering histories [no rain $(\mathrm{NR})$ - open circles, control unsheltered $(\mathrm{CU})$ - grey circles, double rain (DR)- black circles] between years. Values represent change between water-evolved plants and control sheltered plants for each respective treatment (i.e., change between NR-evolved and control sheltered, CU-evolved and control sheltered, and DRevolved and control sheltered).

Figure 4.1: Selection on a trait can be expressed as either directional or non-linear selection. Selection gradients measure the direct effect the trait has on fitness. Selection can affect the distribution of phenotypes in one of three ways: a) directional, b) non-linear, stabilizing, or c) non-linear, disruptive selection. Directional selection $(\beta)$ is the change in the environment favours a single mean trait value, but maintain genetic variation, causing the allele frequency to continually shift in a single direction away from the original mean trait value. Stabilizing selection $(\gamma)$ : a change in the environment decreases the genetic diversity of a population and stabilizes selection on a single particular trait value. Disruptive Selection $(\gamma)$ : a change in the environment favours the extreme phenotypic values of the population rather than an intermediate response. Genetic variation for each extreme phenotypic peak is conserved.

Figure 4.2: Data points represent the mean days to (a) seedling, (b) first flower, and (c) number of seeds per plant, and (d) white flower colour frequency of a population $\mathrm{x}$ genotype combination of wild radish (Raphanus raphanistrum, orange square), hybrid radish (R. raphanistrum $\times R$. sativus, teal triangle), or crop radish (Raphanus sativus, grey circle) grown in one of four locations: Houston, TX, Columbus, OH, Pellston, MI; King City, ON). The black bars represent the mean of all the populations $( \pm \mathrm{SE}$ ) in each location; because of the volume of data, some error estimates are very small. 
Figure 4.3: The strength of directional selection ( \pm SE) imposed on (a) days to seedling emergence and (b) days to flowering across three genotypes (wild radish - Raphanus raphanistrum, orange square; hybrid radish $-R$. raphanistrum $\times R$. sativus, teal triangle; and crop radish Raphanus sativus, grey circle) grown in one of four locations: Houston, TX, Columbus, $\mathrm{OH}$, Pellston, MI; King City, ON. Each data point represents the selection value of a population $\times$ genotype combination across studies. The black bars represent the mean of all the populations

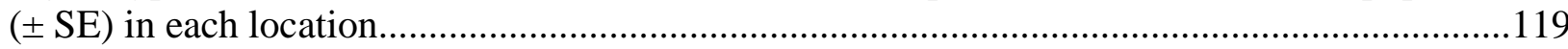

Figure 4.4: The strength of selection differentials (i.e., direct and indirect selection combined) $( \pm$ SE) imposed on (a) days to seedling emergence and (b) days to flowering across three genotypes (wild radish - Raphanus raphanistrum, orange square; hybrid radish $-R$. raphanistrum $\times R$. sativus, teal triangle; and crop radish Raphanus sativus, grey circle) grown in one of four locations: Houston, TX, Columbus, OH, Pellston, MI; King City, ON. Each data point represents the selection differential value of a population $\times$ genotype combination across studies. The black bars represent the mean of all the populations $( \pm$ SE) in each location...................................120 


\section{List of Appendices}

Appendix 1 - Frequency and Volume of Water Applied to Experimental Plots......................130

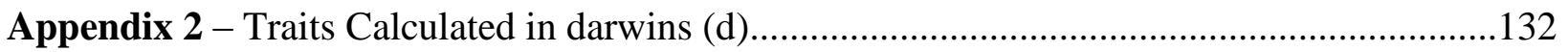

Appendix 3 - Additional Life History Traits Measured......................................................135

Appendix 4 - Reaction Norm Repeated-Measures Analysis...............................................142

Appendix 5 - Divergence Rate Correlations Between Years for Genotype, Historical Watering Environment

Appendix 6 - Source Population Data for Selection Analysis Study.....................................145

Appendix 7 - Error Estimates on Selection Gradient Values in Michigan Populations.............153 


\section{Chapter 1: Hybridization and its Sensitivity to Environmental Context}

\subsection{Premise}

Gene flow is the exchange of genes within and among populations and is one mechanism by which populations can acquire new genetic variation (Rhymer and Simberloff 1996; Sakai et al. 2001; Lee 2002; Crawford and Whitney 2010). Hybridization is a result of gene flow between two genetically distinct species and has been suggested as a mechanism that can enhance or facilitate the invasiveness, or weediness, of a species (Anderson and Stebbins 1954; Barrett 2014; Whitney and Gering 2015; but see Whitney et al. 2010). Invasive species are species that cause sufficient environmental damage through displacing a source species or affecting community dynamics (e.g., reducing diversity or richness) (Colautti and MacIsaac 2004; Leger and Espeland 2010). Further, in the context of agriculture, a plant is classified as a weed if it causes economic damage that affects crop harvest yield (Baker et al. 1965; Lodge et al. 2016). Although there are much broader definitions of invasive weeds, for the purposes of my dissertation, an invasive agricultural weed, is a species of agronomic origin that has spread into a non-agricultural plant community (Richardson et al. 2000). Successful events of gene flow are particularly important in the evolution of invasive species, because gene flow can transfer new trait variation to incipient populations and thus potentially facilitate rapid, adaptive evolution and aid in expansion of the invaded range (Crawford and Whitney 2010; Ridley and Ellstrand 2010; but see Whitney et al. 2010). However, the change in relative fitness of invasive, hybrid populations, as a consequence of acquiring a new adaptive traits, may also be dictated by the environmental context of a population (Whitney et al. 2009; Whitney et al. 2010; Whitney and Gering 2015).

Interspecific gene flow, and the success of hybrid progeny, can be driven by the environment in which plants grow - specifically the abiotic (e.g., temperature, moisture availability) and biotic (e.g., pollinators, con-specific competitors) factors they interact with (Fig. 1.1) (Campbell et al. 2016b). The environmental context may influence hybridization at a number of stages. First, hybridization rates may be altered by proximity of reproductive adults, sharing of pollinators, and the influence of habitats (and their abiotic characteristics, such as temperature and water availability) on plant morphology (e.g., flower size, flower number) which may influence the direction of gene flow or opportunity for mating, and plant phenology 
(e.g., shared flowering schedules) which influences whether species are synchronously fertile) (Anderson and Stebbins Jr 1954; Arnold and Hodges 1995; Bolmgren and Lönnberg 2005; Forrest et al. 2010; but see Campbell et al. 2016b). For example, following a change in land-use, two populations of orchids from different habitats once isolated (prairie species - Cypripedium candidum versus woodland species $-C$. pubescens), now have patches of shared land; this increased gene flow and produced new hybrid varieties (Klier et al. 1991; Vilà et al. 2000). Similarly, gene flow between two Iris species (Iris brevicaulis and I.fulva) from different geographical backgrounds - one arid and one relatively wet - have resulted in a hybrid iris population that occupies a new semi-aquatic niche (Cruzan and Arnold 1993; Arnold and Hodges 1995). Breakdown of physically isolating barriers can increase the number of species that have overlapping phenological cycles. For example, Carpobrotus edulis (characterised by a yellow flower) and C. chiliensis (characterised by a magenta flower) have a large over-lapping flowering period resulting in a successful weedy hybrid with intermediate phenotypes to that of its progenitors (Albert et al. 1997; Vilà et al. 1998; Vilà et al. 2000). Morphological and phenological changes can, in turn, affect biotic interactions with pollinators, which govern gene flow within and among populations (Irwin and Strauss 2005; Brunet and Sweet 2006; Eckert et al. 2009). Considering, the Carpobrotus complexes, changes in phenology and morphology results in all three phenotypes (parental taxa and hybrid) sharing insect pollinators (Vilà et al. 1998). Similarly, parental taxa and hybrids from the Iris species complex also share pollinators (Arnold 1994). Finally, once early-generation hybrid populations are created, the same abiotic and biotic hierarchy can facilitate the persistence and distribution of advanced-generation hybrid populations (Arnold and Martin 2010; Campbell and Wendlandt 2013). For example, Campbell and Wendlant (2013) found a cross of two Ipmosis species from two separate ecotypes (wet ecotype - Ipmosis aggregate, dry ecotype - Ipmosis tenuituba) produced an Ipmosis hybrid variety that had higher stomatal conductance than either parental species; this physiological adaptation may explain the increased persistence and survival of this hybrid relative to its progenitors in patches across the Rocky Mountains. Similarly, Iris hybrids have shown greater relative success depending on the long-term environment they are exposed (Taylor et al. 2009; Arnold and Martin 2010). Environmental variation, therefore, may be a major determinant of both the propensity to hybridize and the relative success of hybrid weeds. 
In this introduction to my dissertation, I will begin by introducing crop-to-wild hybridization (i.e., extreme gene flow) as a phenomenon that can contribute to the evolution of invasive and/or weedy species, but does so inconsistently (Whitney et al. 2009; Whitney et al. 2010). As demonstrated by Fig 1.1, the tendency for hybridization to occur may be, in part, due to the environmental (and specifically abiotic) conditions under which crop and wild plants grow. Furthermore, although the rate at which adaptive evolution can occur is also environmentally dependent (Alberti et al. 2017), phenotypic plasticity may obscure our ability to measure the rate of evolution in these populations. Finally, although natural selection is expected to remove crop-derived traits from weed populations because it is generally expected that crop traits are non-adaptive in weed populations, research on the relative fitness of these traits has revealed cracks in this hypothesis (Jørgensen et al. 1997; Ellstrand et al. 2010; Snow et al. 2010; Ellstrand et al. 2013). Some authors have argued that key domesticated traits may be particularly adaptive for weeds to possess (Gressel 1999, 2005; Campbell et al. 2009a; Snow et al. 2010). I propose an alternative hypothesis: environmental variation changes the strength and direction of selection on crop traits in weed populations and thus environmental variation may create cropwild introgression hotspots across a landscape. Below, I plan to explore themes of the response of advanced-generation hybrid fitness, rates of evolution, and strength of selection to environmental variation, with a special interest in environmental variation in water availability.

\subsection{Hybridization as a Mechanism to Facilitate Adaptive Evolution - Crop-to-wild Gene Flow as an Applied Example}

There are two major hypotheses - transgressive segregation and introgression - that explain why hybridization may radically increase the relative fitness of plant hybrid (Abbott 1992; Rieseberg et al. 1999; Lee 2002; Arnold and Martin 2010; Moran and Alexander 2014; Goulet et al. 2017), particularly during expansion into new environments (Anderson and Stebbins 1954; Arnold and Hodges 1995; Arnold and Martin 2010; Anderson et al. 2011). Through transgressive segregation, the genetic recombination of genes from two genetically distinct parents can generate novel (i.e., new), adaptive phenotypes (Rieseberg et al. 1999; Rieseberg et al. 2003). Introgression, on the other hand, involves the transfer of potentially adaptive traits from one parental population to another (Rieseberg et al. 1999; Arnold and Martin 2010). In both cases, genetic variation increases the probability of producing phenotypes (i.e., traits) well suited to the environment, which aids range expansion or increases competitive vigor 
of the hybrid populations (Anderson and Stebbins 1954; Arnold and Martin 2010). For example, the Louisiana Iris species complex (Iris fluva, Iris hexagona, and Iris brevicaulis) has produced a hybrid lineage (Iris nelsonii) composed of parental traits in a combination that contributes to increased relative fitness as generations post-hybridization increase (Arnold and Martin 2010; Tang et al. 2010). Similarly, changes in relative success of hybrids has been observed in natural populations of Ipomosis species, as well (Campbell and Waser 2007; Campbell and Wendlandt 2013). Therefore, in natural systems without human interference, hybridization has been observed as a naturally occurring evolutionary phenomenon to promote increased relative fitness and survival.

Hybridization of crops and their wild relatives is a well-studied area in the application of hybridization as a mechanism to promote rapid adaptive evolution. Crop-to-wild gene flow has produced aggressive, environmentally-damaging weeds under variable environments (Snow and Campbell 2005; Campbell et al. 2006; Hovick et al. 2012: Ellstrand et al. 2013; Hovick and Whitney 2014; Whitney et al. 2015; but see Whitney et al. 2010). Crop-to-wild gene flow is often thought of as a unidirectional process since agronomic industries have strict phenotypic standards on crop seed lots; therefore, seeds that do not meet specified criteria are discarded (Stewart Jr et al. 2003; Snow and Campbell 2005; Warwick and Stewart 2005). Additionally, weeds that grow in crop fields are selectively removed before gene flow has a chance to occur (Snow and Campbell 2005); although the opportunity for wild-to-crop is available, it is rare for a plant, let along populations, to survive and persist. When crops mate with wild relatives, they can transfer crop-derived alleles to wild populations. Crop traits, are often perceived to be negatively associated with fitness (Gressel 1999; Jenczewski et al. 2003; Gressel 2005), and yet persist in wild populations (Snow et al. 2010; Campbell et al. 2016a). For example, transgenic crop populations have been known to transfer Bt-genes (confer insect-resistance) and glyphosateresistance (herbicide) in sunflower (Helianthus species) and kochia (Kochia species) plants, respectively, in wild populations of related species; the resulting hybrids demonstrate strong selection for these traits (Snow 2002; Beckie et al. 2013). Furthermore, the diverse gene pool of hybrid populations may allow populations to respond more dynamically to environmental variation, with hybrid lineages known to evolve more rapidly than non-hybrid lineages in response to the same environmental strength of selection (Campbell et al. 2009b; Ridley and Ellstrand 2010; Anderson et al. 2011). There is a significant body of research on crop-wild 
hybridization (Ellstrand 2003; Schierenbeck and Ellstrand 2009; Ellstrand et al. 2010; Goulet et al. 2017) and its ability to facilitate weediness in some, but not all, environments. However, there is a considerable knowledge gap that identifies the underlying abiotic pressures that promotes hybridization, the relative success and persistence of hybrids.

One example of a hybrid system that has shown varying success based on its ecological context is crop-wild hybrid radish (i.e., Raphanus species-complex). The greatest relative success of hybrid populations was documented in California where hybrid radish (also referred to as California wild radish or wild $R$. sativus) populations were far more fecund that progenitors, and have replaced naturally occurring wild radish (jointed charlock or $R$. raphanistrum) in the area (Hegde et al. 2006). However, this level of relative success has not occurred every location where the plant has been experimentally introduced. For example, in Michigan environments, populations of hybrids were, at times more fecund or equally as fecund as wild populations (Campbell et al. 2006; Campbell and Snow 2007). Similarly, hybrid performance in Ontario equalled that of wild populations (Teitel et al. 2016a). When introduced into Texas, a novel environment (i.e., a new environment where radish has not been documented to grow) similar to California, hybrid populations again outperformed wild populations (Hovick et al. 2012). Finally, in a study conducted by Campbell et al. (2016), found that water availability, did not affect the production of $\mathrm{F}_{1}$ crop-wild hybrids. One of the most apparent differences among these studies (Ontario, Michigan, California, and Texas; Campbell et al. 2006; Hovick et al. 2012; Teitel et al. 2016) was natural rainfall and temperature cline. Michigan and California studies had similar cumulative rainfall but had temporal variability (according to Weather Underground), where Michigan had evenly-distributed rainfall while rainfall in California occurred mostly within the first two months of the growing season (Campbell et al. 2006, Table 1.1). Texas had the highest temperatures and lowest cumulative rainfall across all four locations (Table 1.1). Ontario in comparison to all sites, resembled Michigan conditions, with similar accumulative rainfall and seasonal patterns. Although variation in climate exists between experiments, studies explicitly evaluating how abiotic variation influences the persistence and invasiveness of late-generation hybrids are needed to assess the possibility that conclusions from one hybrid population in one location could apply to another hybrid population in other moisture conditions. 


\subsection{Rates of Evolution as a Metric for Measuring Change}

Not only can environmental variation influence the rates of hybridization and introgression, but also our ability to measure the rate at which populations evolve. Before, I reveal how this is true, I first will explain how evolutionists measure evolutionary rates. Rates of evolution are measured using metrics that assess the degree of phenotypic change relative to phenotypic variation and are commonly measured in darwins $(d)$ or haldanes $(h)$ (Haldane 1949; Gingerich 1983, 1993; Bone and Farres 2001; Campbell et al. 2009b). Both of these metrics measure the natural log mean phenotypic difference (i.e., $\Delta \overline{\mathrm{x}}$ ) between contemporary and ancestral populations through time (Haldane 1949; Bone and Farres 2001). The two key differences between these two metrics lies in how time is measured: 1) time in darwins is measured as the elapsed time in powers of $e$ per millions of years and haldanes are measured as the evolutionary change across generations (i.e., a plant's life cycle) and 2) haldanes standardize the difference in means by the pooled variance. One of the most comprehensive reviews of evolutionary rates in plants was done by Bone \& Farres (2001). They calculated and summarized evolutionary rates of multiple plant species in response to natural and artificial selection, collected from published examples of plant evolution. For example, Bone and Farres (2001) calculated minimal evolution of biomass between invasive populations of Lythrum salicaria (Blossey and Notzold 1995), in the presence and absence of natural herbivory over 150 years. Similar low rates of evolution over 50 to 100 years, with respect to shifts in biomass selection, were found with several invasive species when comparing between native and introduced ranges. In contrast, high rates of evolution in only nine generations were documented in Avena sativa populations that were artificially selected for high oil content (Frey and Holland 1999), and modest rates of evolution in oil content were documented in Zea mays (corn) over 28 to 100 generations (Dudley 1977; Dudley and Lambert 1992; Lambert et al. 1997; Bone and Farres 2001). Crop-wild hybrids have also shown variable rates of evolution, ranging from slow rates in natural Helianthus hybrid populations over 50 years (Carney et al. 2000; calculated by Bones and Farres 2001) to relatively fast for artificially selected traits in hybrid Raphanus populations (Campbell et al. 2009b). These calculated rates are helpful in describing the evolutionary trajectory of a species and potentially predicting the consequences of global change. A major determinate in the phenotype is environmental variance component $\left(\mathrm{V}_{\mathrm{E}}\right)$. However, a serious flaw presents itself in which the theory (and thus calculations) does not account for 
environmentally induced variations in phenotype. Thus, the predictions of evolution that arise from studies may be limited in their utility. I explore the consequences of this flaw in the accuracy of evolutionary rate estimates and discuss solutions to correct for this inaccuracy in Chapter 3.

\subsection{Geographic Variation in the Success of Hybrid Plants}

Hybridization may facilitate gene flow but natural selection will govern the likelihood that a crop trait will persist in a population if it confers a reproductive advantage. The strength and direction of selection a population experiences will depend on the ecological context of the population. Advantageous traits for agricultural weeds may include those that confer herbicide (e.g., glyphosate) or insecticide (e.g., Bt gene) resistance. The transfer of the $B t$ gene into sunflower hybrid populations or Brassica populations, for example, have resulted in increased fecundity in crop-wild hybrid populations when exposed to herbivory compared to wild progenitors; however, these gene transfer events did not offer a fitness advantage or cost when herbivores were absent (Stewart et al. 1997; Snow et al. 2003; Chapman and Burke 2006). Similarly, when measuring selection across environments, selection on crop and wild traits varied in crop-wild hybrid sunflower populations grown in Indiana versus Nebraska (Baack et al. 2008); however, the selection pressures that may be facilitating these differences were not identified. Researchers that have measured the general phenomenon of crop-wild hybridization across environmental clines have revealed that the success and strength of hybridization is geographically variable (Hegde et al. 2006; Martin et al. 2006; Campbell and Waser 2007; Whitney et al. 2009; Campbell and Wendlandt 2013; Campbell et al. 2014). Additionally, environmental variation clearly affects the strength and direction of selection on hybrid populations, which may be one mechanism by which relative fitness of hybrid population's changes across landscapes. Although variation in the expression of crop traits has been observed, selection on crop traits in hybrid populations have not been explicitly quantified. In chapter 4, using a phenotypic selection analysis, I measure the strength of selection on crop traits across a geographic landscape to determine if crop traits persist in a similar fashion. 


\subsection{Experimental Environmental Clines as an Evolutionary Tool}

To help discern the underlying mechanisms that promote population change, scientists sometimes use experimental environmental clines, such as temperature, water availability, or $\mathrm{CO}_{2}$ concentration gradients that are artificially imposed on plant populations. For example, heating arrays can be used to manipulate temperatures (Wadgymar et al. 2015) or open-top $\mathrm{CO}_{2}$ chambers (Davey et al. 1999; Pleijel and Högy 2015) to manipulate $\mathrm{CO}_{2}$ levels. Water is an important resource that can aid in the success of plant populations, whereby increased wateravailability can increase plant-productivity (Michaletz et al. 2014). Although, water-availability can easily be controlled under laboratory conditions, laboratories lack natural lighting, pest populations, and temperature cycles. Rain-out shelters can be used in-field and have been used in a variety of work aimed at measuring drought impacts in crops or desert species, measuring nutrient changes and physiological changes (Fay et al. 2000; Yahdjian and Sala 2002). In this work, using rain-out shelters based on designs inspired by Yahdjian and Sala (2002) I have attempted to create extreme moisture clines that would represent a wide diversity of rainfall scenarios: from extremely dry to extremely wet scenarios. Our shelters were built $3.05 \mathrm{~m}$ by 2.44 $\mathrm{m}$ wooden frames with transparent sheet plastic stretched over the frame, acting as a roof. Using metal poles, frames were slanted and elevated to approximately $1.2 \mathrm{~m}$ above ground at their lowest corner. Frames were slanted in order to intercept and divert natural rainfall into a $208 \mathrm{~L}$ plastic collection barrel via an eavestrough attached to the lowest side of the wooden frame. Although these roofs divert rainfall, other environmental conditions also changed, including light quality and quantity (because the translucent roofs block UV; Campbell, unpub. data).

\subsection{Objectives}

Previous experimental research revealed the significant contribution of hybridization to adaptive evolution and success of weedy plants (Campbell et al. 2006; Teitel et al. 2016a; Teitel et al. 2016c). Moreover, because hybridization can lead to dramatic improvements in relative fitness, crop alleles can persist for long periods of time in weedy populations (Snow et al. 2010) and evolution proceeds much more quickly in hybrid than non-hybrid populations (Anderson and Stebbins Jr 1954; Rieseberg et al. 1999; Rieseberg et al. 2003; Campbell et al. 2006; Campbell and Snow 2009; Campbell et al. 2009b). Despite numerous studies of the evolution of crop-wild hybrids and that document the persistence of crop alleles in wild populations (Campbell and 
Snow 2009; Snow et al. 2010; Hovick et al. 2012; Hovick and Whitney 2014; Whitney et al. 2015), the ecological contexts that can influence the relative success of these crop-wild hybrids in weedy, or invasive, populations is still unexplored. My research took the study of hybridization in several new directions by exploring how experimentally manipulated environmental variation affects the success and strength of selection in advanced generation crop-wild hybrids.

In Chapter 2, common garden experiments tested novel questions on the influence of source versus sink environmental conditions on the success of invading species. Rather than treating the process of gene flow as a 'black box,' these experiments tracked changes in the crop allele frequencies and measure plant responses to soil moisture variation. From this work, I discovered that seedling emergence of Raphanus may be shaped by both genetic predisposition and the environment of the source population and the current environment plants are grown. In Chapter 3, I explored the susceptibility of rate of evolution metrics to distortion by the environmental sensitivity of traits. I compared estimates of the rate of evolution of 20 wild and 20 hybrid experimental populations grown in two common gardens after the 40 populations had evolved for three generations under either relatively dry, relatively wet or control experimental conditions. Morphological differences between these replicated lineages are expected to reflect divergent evolutionary paths, when grown in a common garden. However, the environment of the common garden itself influenced trait expression. This work highlighted a major weakness in current rate of evolution metrics and built on the existing theory to solve the problem of environmental variation on trait expression. Finally, in Chapter 4, using a meta-analytic approach using data collected over the last twelve years and four locations, I uncovered a significant amount of variation in the strength and direction of selection on crop traits expressed in weedy populations. Chapters 2, 3 and 4 of this dissertation is organised and formatted as manuscripts for submission. By completing this large, integrated study, this work can offer a new perspective on the abiotic pressures that affect the persistence of advanced-generation crop-wild hybrid populations and their ability to generate weeds.

\subsection{The Raphanus Crop-Wild Complex}

During my dissertation, I studied cultivated (Raphanus sativus L.) and wild radish or jointed charlock (Raphanus raphanistrum L.); genetically distinct but related species (Lewis- 
Jones et al. 1982; Yamagishi and Terachi 2003; Yamane et al. 2005). Cultivated radish originated in Egypt and Pakistan (Snow and Campbell 2005; Yamane et al. 2005) and wild radish is native to Europe and introduced into California more than a century ago (Panetsos and Baker 1967; Yamagishi and Terachi 2003; Yamane et al. 2005). Wild radish (R. raphanistrum), or jointed charlock, has a long-lived seed bank, a smaller taproot system with narrow branching roots, asynchronous seedling emergence schedules after tilling, and early flowering phenology (Conner and Via 1993; Holm 1997; Sahli et al. 2008). Wild fruits have a woody, tough coating that fall from the plant at maturation and break into small segments; the coating prevents immediate germination and a level of dormancy into the next season (Reeves et al. 1981; Snow and Campbell 2005). It is homozygous recessive for the yellow flower colour phenotype (Panetsos and Baker 1967). Plants are often found in agricultural field margins, disturbed areas, and coastal beaches (Holm et al. 1997, Snow and Campbell 2004). Wild radish is reported as a globally distributed, harvest damaging weed in more than 45 agronomic systems in at least 65 countries, including North and South America, Japan, and Australia (Holm 1997; Ellstrand et al. 2010; Bhatti et al. 2016; Han et al. 2016); demonstrating the weed's vast dispersal and competitive ability.

Crop radish (R. sativus) is thought to be derived from several wild varieties including $R$. raphanistrum, $R$. maritimus, and may have also be a hybrid derived from $R$. landra $\times R$. maritmus cross (Lewis-Jones et al. 1982; Yamagishi and Terachi 2003; Yamane et al. 2005); crops have several documented origins including use in Egypt, and Pakistan (Snow and Campbell 2005). Cultivated radish has a short-lived seed bank, has a large edible root system, early seedling emergence times, high seed production, and flowers later in the growing season (Snow and Campbell 2005). Upon maturation, crop fruits remain on the plant and seeds are easier to harvest since fruits have a softer and easily breakable husk. Crop plants are characterised by a homozygous dominant for the white flower colour phenotype. Crop radish can hybridize with many other crops including several Brassica species (Scheffler and Dale 1994; Liu et al. 2003) but most readily, and commonly, hybridize with wild $R$. raphanistrum.

Crop and wild radish share several traits that allow them to cross-fertilize and hybridize. Specifically, they both belong to the Brassicaceae family, have annual growth cycles, are insect pollinated, and self-incompatible diploid species (Panetsos and Baker 1967; Snow et al. 2001). Successful crop-wild radish hybrid populations have been documented in South America 
including Argentina, Brazil, and Chile (Ellstrand et al. 2010; Pandolfo et al. 2016) with North America, having varying success of hybrid radish in Michigan, Texas, Ontario, and California. Crop allele introgression in these populations may be simply tracked with a visible trait - flower petal colour, with white petal colour allele exhibiting simple Mendelian dominance over the yellow flower colour allele. The first generation of heterozygote hybrids will have exclusively white flowers, whereas the second generation of segregating hybrids is expected to express a 3 white: 1 yellow flower petal ratio (Panetsos and Baker 1967). Additionally, the first generation of crop-wild radish hybrids tend to abort more than $50 \%$ of their pollen grains due to the genetic recombination between nonhomologous chromosomes (i.e., reciprocal translocation process, because crops have 22 chromosomes and wild radish have 23 chromosomes; Panestos and Baker 1976). However, populations of hybrid radish can quickly regain fertility (Campbell et al. 2009b), produce competitively robust plants (Campbell \& Snow 2007) and improve the relative success of weedy radish populations. However, as discussed above, the success of hybrid-radish weed populations seems to be unique to specific environments. Although radish are not current candidates of genetic engineering, the Raphanus species complex has been used as an ecological model for studying the introgression of transgenes (Klinger et al. 1991; Snow et al. 2001). Raphanus species have relatively short growth cycles and a mating system representative of a variety of cultivated species that are genetically engineered including, but not limited to, certain grains (e.g., rye, buckwheat), vegetables (e.g., cabbage, cucumber, carrots), oilseed (e.g., canola) and forage crops (e.g., alfalfa), to name a few (Klinger et al. 1991; Hancock 2012); with all listed examples having demonstrated high inter-fertile gene flow with wild relatives via insectmediated pollen transfer (Ellstrand et al. 1989; Klinger et al. 1991). Considering radish is a lowpriority transgene-receiving candidate, has a short generation time, and represents a mating strategy common to many crops, the Raphanus species complex is an ideal system to evaluate crop-to-wild gene flow. 


\subsection{Table List}

Table 1.1: Minimum, average, and maximum temperature and cumulative data in studies Campbell et al. 2006 (Michigan and California), Hovick et al. 2012 (Texas), Teitel et al. (2016) and this study (Ontario). The city and weather station code are presented in parenthesis after each location. The data presented are calculated from the growing season of each study: Michigan (May - September), California (January - June), Texas (February - July), and Ontario (May September). Data for studies in the USA (Michigan, California, and Texas) were collected from the Weather Underground website (www.wunderground.com) while Ontario data was collected from the Government of Canada website (www.climate.weather.gc.ca).

\begin{tabular}{lcccc}
\hline Location and Year & $\begin{array}{c}\text { Min. Temp } \\
\left({ }^{\circ} \mathbf{C}\right)\end{array}$ & $\begin{array}{c}\text { Avg. Temp } \\
\left({ }^{\circ} \mathbf{C}\right)\end{array}$ & $\begin{array}{c}\text { Max. Temp } \\
\left({ }^{\circ} \mathbf{C}\right)\end{array}$ & $\begin{array}{c}\text { Cumulative } \\
\text { Rainfall }(\mathbf{c m})\end{array}$ \\
\hline Michigan (Pellston - KPLN) & & & & \\
\hline 2005 & -7.2 & 17.2 & 35.0 & 33.8 \\
\hline California (Riverside - KRAL) & & & & \\
\hline 2005 & 2.2 & 15.9 & 36.1 & \\
\hline Texas (Katy - KTME) & & & & \\
\hline 2010 & -2.2 & 20.9 & 35.6 & \\
\hline Ontario (Markham - YKZ) & & & & \\
\hline 2013 & 1.1 & 18.1 & 35.6 & 52.8 \\
2014 & 1.5 & 17.6 & 30.5 & 38.5 \\
2015 & 2.0 & 20.2 & 34.3 & 21.6 \\
2016 & 0.7 & 19.6 & 35.1 & \\
\hline
\end{tabular}




\subsection{Figure List}

\section{Abiotic Factors}

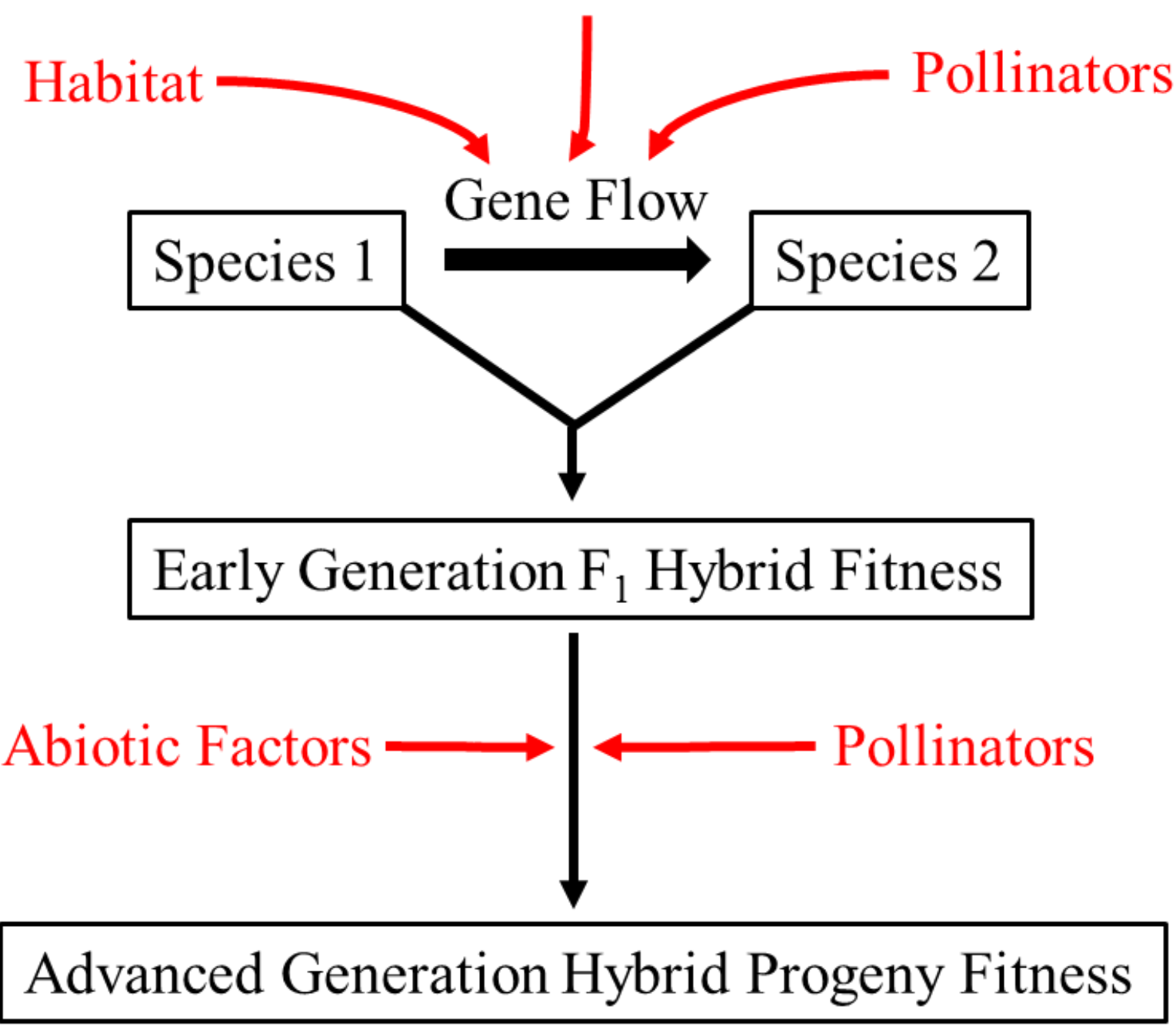

Figure 1.1: Interspecific gene flow, and successive hybrid progeny, can be driven by the environment plants are found in and the abiotic (e.g., temperature or moisture availability) and biotic (e.g., pollinators, con-specific competitors) factors they interact with. Hybridization rates, firstly, may be altered by the habitats progenitor species are found in, where changes in abiotic and biotic factors will affect the propensity for hybridization to occur. Once early-generation hybrid populations are created, the same abiotic and biotic hierarchy can facilitate establishment and the spread of advanced-generation hybrid populations. 


\section{Chapter 2: Evolution of Advanced-Generation Hybrids After Climate Change and Crop Gene Introgression Does Not Increase Weediness of Crop-Wild Hybrids}

\subsection{Abstract}

Crop-to-wild hybridization, the directional transfer of crop genes into wild populations, can generate unique genotypes, populations of which can adaptively evolve to produce successful invasive organisms. Only a fraction of hybrid populations are genetically predisposed to successfully invade a novel environment but do the environmental conditions of the source and sink populations also affect the degree of hybrid invasion success? For four generations, 20 wild radish (Raphanus raphanistrum) and 20 hybrid radish ( $R$. sativus $\times R$. raphanistrum) plant populations evolved under experimentally-manipulated moisture conditions (dry, wet, or control; i.e., their soil moisture history) in old fields near Toronto, Canada. Then, I planted these advanced-generation wild and hybrid radish plants in sheltered experimental plots that were also exposed to dry, wet, and control conditions. Hybridization produced larger plants across all environments and, in wet environments, hybrid plants had higher quantum PSII efficiency, a trait correlated with photosynthetic activity. Additionally, low current moisture had an effect on seedling emergence time. Although hybridization and soil moisture affected plant growth and physiology, this did not translate into fecundity differences between wild and hybrid plants or between historic or current watering environments. The persistence of crop-derived traits was not influenced by soil moisture environments. Hybridization and watering history did not enhance the success of advanced-generation hybrid plants relative to wild progenitors in Ontario, Canada. Thus, risk assessment of the invasion success of weedy radish populations may need to focus on other environmental clines.

Key words: Rain-out shelters, hybridization, moisture availability, invasion, radish *Intended for submission to American Journal of Botany (AJB - ISSN: 1537-2197) 


\subsection{Introduction}

Hybridization, inter-specific mating, of crops and their wild relatives can produce aggressive, environmentally-damaging weeds in a diversity of environments (Campbell et al. 2006; Ridley and Ellstrand 2008; Arnold and Martin 2010; Hovick et al. 2012; Ellstrand et al. 2013; but see Whitney et al. 2010). When crops mate with wild relatives, they can transfer cropspecific alleles to wild populations; these crop alleles can persist and contribute beneficial adaptive phenotypes that can be selectively advantageous (Campbell et al. 2006; Whitney et al. 2006; Snow et al. 2010). Hybridization may lead to heterosis (hybrid vigor) by masking deleterious, recessive alleles through additive dominance or transfer adaptive crop alleles to weed populations (Rieseberg and Carney 1998; Barton 2001; Whitney et al. 2006) - increasing the relative success of hybrid plants. Additionally, the combined crop and wild gene pool can harbour increased genetic variation and aid in more dynamic responses to environmental variation (Ludwig et al. 2004; Mallet 2007; Warwick et al. 2009; Hovick et al. 2012; Goulet et al. 2017). Although there is considerable research on crop-wild hybridization and its influence on weediness, there is a considerable gap in our knowledge: how does environmental variation influence the persistence and success of late-generation hybrids?

Hybrid performance is not consistent across environments and variable success may be driven by the ecological context where the population grows. For example, the transfer of the $B t$ gene (i.e., confers insect resistance) in sunflower hybrid populations and Brassica populations have demonstrated increased fecundity in crop-wild hybrid populations when exposed to herbivory compared to wild progenitors; however, it did not offer a fitness advantage or cost when plants were grown in the absence of herbivores (Stewart et al. 1997; Snow et al. 2003; Chapman and Burke 2006). Similarly, when measuring selection in Indiana versus Nebraska, selection on crop and wild traits varied among crop-wild hybrid sunflower populations (Baack et al. 2008); however, the selection pressures that may be facilitating these differences were not directly identified. Similarly, researchers measuring crop-wild hybridization in radish plants across environmental clines have demonstrated geographic variation in the success of hybrids and strength of selection on these populations (Whitney et al. 2009; Campbell et al. 2014). Successful crop-wild radish hybrid populations have been documented in South America including Argentina, Brazil, Chile, and Australia (Ashworth et al. 2016; Pandolfo et al. 2016; Heap 2018) with North America, having varying success of hybrid radish in Michigan, Texas, 
Ontario, and California (Campbell et al. 2006; Hovick et al. 2012; Teitel et al. 2016). One of the most notable differences among North American populations was natural rainfall and temperature across a growth season (see Chapter 1 - Table 1.1). Michigan and California studies had similar cumulative rainfall but had temporal variability, where Michigan had evenlydistributed rainfall while rainfall in California occurred mostly within the first two months of the growing season (Campbell et al. 2006, Table 1.1). Texas had the highest temperatures and lowest cumulative rainfall across all four locations (Table 1.1). Ontario in comparison to all sites, resembled Michigan conditions, with similar accumulative rainfall and seasonal patterns (Table 1.1). Although variation in climate exists between experiments, studies explicitly evaluating how abiotic variation influences the persistence and success of late-generation hybrids are rare and necessary to assess the predictability of relative hybrid success across moisture conditions.

Changes to soil moisture have routinely influenced the phenology, morphology, and persistence of plant populations (Barrett and Harder 1996; Halpin 1997; Parmesan and Yohe 2003; Charlesworth 2006). Compared to progenitor species, hybrid populations of Ipomopsis aggregata $\times I$. tenuituba produced appreciably more flowers under experimentally dry moisture conditions but not under moist conditions (i.e., ambient and experimentally watered), (Campbell and Wendlandt 2013). This pattern, where hybrid plant populations perform better than progenitors under extreme watering conditions is common in the literature (Abbott et al. 2003; Brock and Galen 2005; Wu and Campbell 2006; Ma et al. 2010). Conversely, moisture gradients have also been shown to not affect the propensity for hybridization to occur (Campbell and Waser 2007; Whitney et al. 2010; Campbell et al. 2016b). Our knowledge of how long-term environmental pressures, specifically water availability, influence crop-to-wild gene flow and consequently the persistence of these traits in advanced generations is still a relatively young area of research.

Climatic variation among spatial locations has a substantial impact on evapotranspiration rates in plants (Mooney and Gulmon 1979). Water is a limiting factor in many environments and the moisture deficit (in plants or in the soil) can affect plant stomatal conductance and gasexchange rates (i.e., carbon dioxide and oxygen), and ultimately influence photosynthetic capacity and activity (Sherrard and Maherali 2006; Bresson et al. 2015; Goltsev et al. 2016). Plants need to successfully minimize water loss, maintain optimal transpiration rates (i.e., gasexchange) to drive photosynthesis, but avoid desiccation in response to moisture deficits. This is 
most successfully accomplished by plants that evolve escape, avoidance, or tolerance strategies (Levitt 1980; Chaves et al. 2003). Escape strategies enlist a high level of plasticity that require successful reproduction prior to the onset of drought. This can be accomplished through fast growth cycles and metabolic activity that utilize resources while water lasts and is primarily exhibited by plants in arid or desert regions (Chaves et al. 2003). Plants that avoid dehydration maintain lower metabolic activity to decrease resource demands. This strategy requires minimal water loss (via closed stomates or reduced leaf surface area) and maximal water-uptake, for example through increased rooting depth or leaf shedding (Chaves et al. 2003; Sherrard and Maherali 2006). Lastly, tolerance to low tissue water pressure which may occur via changes in cell size, more rigid cell walls, or shifts in osmotic potential is a common response in evergreens species (Chaves et al. 2003). Natural selection should favour conservative water-use through one, or a combination, of these strategies if it is adaptive.

Evapotranspiration rates can vary between crop and wild plants. Many crop plants are sensitive to water stress and, therefore, are under strict agronomic control (Sayed 2003; Baker and Rosenqvist 2004). For example, soybean plants [Glycine max (L.) Merr.] biomass and photosynthetic rates decreased in response to short term drought conditions relative to plants in well-watered environments (Ohashi et al. 2006). Similarly, many breeders have developed new drought resistance varieties of crops, due to their low drought-tolerance, including varies of maize (Saccardy et al. 1998) and wheat (Balota and Lichtenthaler 1999). Wild plant populations are more commonly exposed to environments with insecure or inconstant soil moistures (Jenczewski et al. 2003; Warwick and Stewart 2005) and thus may be better adapted to drought or routine inundation, and as such, may possess traits that allow for a more robust physiological response to water stress than crop species (Chapin III et al. 1993; Johnson et al. 2000). However, modern crops also possess a suite of stress-tolerant traits, including tolerance to limited water availability (Shisanya 2002); at times acquiring these traits from wild relatives (Johnson et al. 2000; Warschefsky et al. 2014; Becklin et al. 2016). For example, sunflower cultivars (Helianthus annuus L.) with introgressed traits from Helianthus argophyllus L.) possess higher water-use efficiency (WUE) in drought conditions than traditional cultivars (Baldini and Vannozzi 1999; Rauf 2008). Several physiological correlates of evapotranspiration exist to provide insight into plant water-use efficiency (Chaves et al. 2003). Chlorophyll fluorescence, a measure of photosystem II efficiency (PSII), a correlate of a plant's tolerance to environmental 
drought stress (Maxwell and Johnson 2000; Favaretto et al. 2011). Similarly, leaf size and biomass can provide indirect insights into the water-use efficiency of the plant (Dudley 1996; Donovan et al. 2007). For example, Dudley (1996) found that intermediate leaf area was positively correlated with high WUE and higher biomass in a wildflower, Cakile edentula var. lacustris. Similarly, others found a relationship between optimal leaf size and WUE, depending on environmental context (Baldini and Vannozzi 1999; Ludwig et al. 2004; Donovan et al. 2011). Biomass, therefore, may be useful tool as an indicator of water use efficiency and reproductive output and help determine the relative importance of hybridization and environmental context (i.e., selection history) on the success of invasiveness.

\subsubsection{Objectives}

To investigate how variation in soil moisture may influence the phenotype, physiology, and fecundity of crop-wild hybrids, I established 40 experimental populations of wild and cropwild hybrid radishes across an old field in Newmarket, Ontario, Canada and manipulated moisture availability using a series of rain-out shelters. To measure the evolution of survival and fecundity of advanced-generation hybrids, I grew fifth-generation $\left(\mathrm{G}_{5}\right)$ wild and hybrid plants in sheltered common gardens, watered with low, ambient, or high soil moisture in Ontario in 2015 and 2016. Given that earlier studies have detected hybrid success when introduced into novel (i.e., new) environments (Hovick et al. 2012, Campbell et al. 2006) across natural moisture gradients, I explored the evolutionary processes and moisture conditions that influence long-term crop allele persistence in and fitness of advanced-generation hybrid weed populations. Finally, I measured the relative importance of hybridization and selection (imposed by soil moisture) on the success of weedy radish invasiveness. Here I asked:

(1) Does the relative success of advanced-generation wild and hybrid plants differ across a soil moisture gradient?

(2) Does the frequency of crop-derived, simply inherited alleles differ among hybrid populations grown under one of four soil moisture treatments?

(3) Does soil moisture affect relative hybridization success by altering complex (i.e., not simply inherited via Mendelian genetics), quantitative plant traits (including physiological function, life history, or phenology)? 
In observing flower petal colour frequency as a crop-derived marker and a simply inherited dominant trait; based on Hardy-Weinberg equilibrium, I anticipated white petal colour frequency in hybrid populations would be not significantly different than $75 \%$. Furthermore, I did not anticipate flower colour to vary between environments. With respect to more complex, quantitative traits, I expect wild radish plants will be better adapted and will be relatively more successful than hybrid plants when exposed to extreme soil moisture. 


\subsection{Methods}

\subsubsection{Study Species}

Cultivated (Raphanus sativus L.) and wild radish (jointed charlock; Raphanus raphanistrum L.) are annual, insect-pollinated, self-incompatible diploid species that can hybridize (Panetsos and Baker 1967). Cultivated radish is an annual, crop species that flowers late, has increased seed production, and large edible hypocotyls (i.e., roots) (Snow and Campbell 2005). Wild radish, in contrast, has long-lived seed banks, a smaller taproot system with narrow branching roots, early germination times after tilling, and earlier flowering than $R$. sativus. Wild radish is a common, damaging weed in agricultural systems but can be found in disturbed and costal sites in temperate climates (Holm 1997). Hybrid radish (Raphanus raphanistrum $\times R$. sativus) is an economically-damaging weed, particularly in California where all populations of wild radish have been replaced by hybrid populations (Ridley and Ellstrand 2010).

\subsubsection{Study Site and Seed Sources}

Parental wild and crop populations were grown at Ohio State University's Waterman Farm research station, Columbus, $\mathrm{OH}, \mathrm{USA}\left(40^{\circ} 0^{\prime} \mathrm{N}, 83^{\circ} 1^{\prime} \mathrm{W}, 232 \mathrm{~m}\right.$ asl) in 2010. The research station was in a temperate region with average summer temperatures reaching between $19^{\circ} \mathrm{C}$ to $25^{\circ} \mathrm{C}$ between the months of May to August and total annual precipitation reaching 921 $\mathrm{mm}$. Ancestral populations (i.e., $\mathrm{F}_{0}$ generation) of wild radish (Raphanus raphanistrum) were collected from greenhouse populations that were grown for several generations near Binghamton, NY, USA (Conner and Via 1993). The crop radish (Raphanus sativus) cultivar used was Red silk from the Harris-Moran Seed Company in Modesto, CA, USA. As described in Campbell et al. (2016b), in 2010, both cultivated and wild plants (nine seedlings per genotype) were planted in 36 plots as part of a randomized block design at the Waterman Farm at Ohio State University in Columbus, Ohio USA. Seedlings were planted in one of four watering treatments with one plot per treatment, per block, for a total of ten blocks. Each of these plots were approximately 200 meters apart to prevent gene flow among plots (Klinger et al. 1991) and promote gene flow between species within plots. Pollinator-mediated gene flow between plants may occur at greater distances (Klinger et al 1991, Pasquet et al. 2008); however, the frequency of pollen exchange and hybridization success sharply declines beyond one meter from the plant (Klinger et al. 1991). Plots were exposed to one of four historic watering treatments: 
(1) Control Unsheltered (CU): To determine how precipitation affects wild and hybrid plants, ambient rainwater fell naturally on these un-manipulated plots;

(2) Control Sheltered (CS): To determine if shelter presence had an effect on plant growth, ambient rainwater collected in CS shelter barrels were applied to the plot;

(3) No rain/low rain (NR): To determine how the lack of precipitation affected plant growth, water collected from NR shelter barrels were not applied to NR plots. Small amounts of rain may have entered through open sides of the plot, nonetheless, NR plots remained relatively dry;

(4) Double Rain (DR): To determine how excess precipitation affected plant growth, water collected from DR and NR shelter barrels were applied to DR plots; that is, double the ambient rainfall (Campbell et al. 2015).

Gene flow naturally occurred within mixed plots of wild and cultivated plants which gave rise to first-generation (i.e., $\mathrm{F}_{1}$ ) wild and crop-wild hybrid ( $R$. sativus $\times R$. raphanistrum) seeds. Succeeding generations $\left(\mathrm{G}_{1}\right.$ to $\left.\mathrm{G}_{4}\right)$ from 2011-2014 were grown at the Koffler Scientific Reserve

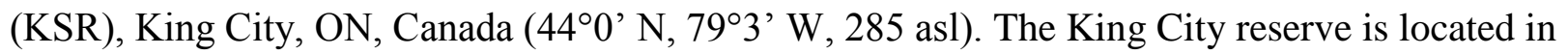
the Oak Ridges Moraine, a temperate region of headwaters, rolling hills and valleys. This area consists of pasture and woodland sites that are exposed to natural weather patterns, herbivory, and pollinators. Average temperatures over the four years ranged from $14^{\circ} \mathrm{C}$ to $23^{\circ} \mathrm{C}$ between May to August, with total precipitation of $349.8 \mathrm{~mm}$ in $2011,358.4 \mathrm{~mm}$ in 2012, $448.5 \mathrm{~mm}$ in 2013, and $416.6 \mathrm{~mm}$ in 2014 during these months (according to the nearest weather station: Buttonville, Ontario 4351'39" N , 79²2'07" W; Government of Canada 2018). During my 2015 and 2016 common garden experimental years, natural rainfall varied over the growing season between common garden years, with a cumulative rainfall of approximately $307.8 \mathrm{~mm}$ in 2015 and $206.7 \mathrm{~mm}$ in 2016.

\subsubsection{Establishment of Replicated Populations under Watering Treatments and Common Garden} Seed Sources

Once relocated to $\mathrm{KSR}, \mathrm{F}_{1}$ seeds from 40 randomly-chosen maternal watering environments - five plots per water treatment per wild or hybrid genotype - and grown in germination trays in a hoophouse located at the reserve. Seedlings were grown to the two-leaf stage and transplanted into plots across KSR that corresponded to the seedling's maternal 2010 
watering environment (CU, CS, NR, DR) using rain-out shelters. Each of the four watering treatments had five replicate populations for a total of 20 populations for hybrid plants and a total of 20 populations of wild plants. Shelters were placed at least $40 \mathrm{~m}$ apart to prevent gene flow among plots and promote gene flow within plots. The watering treatments imposed on plants from 2010 to 2014 will, hereafter, be referred to as the plants' historical watering treatment. The opportunity for selection did not exist in the first generation $\left(F_{1}\right)$ because they were essentially a single genotype (Campbell et al. 2016b) and with limited variation in traits (i.e., low genetic variation). Small population in the $\mathrm{G}_{2}$ and $\mathrm{G}_{3}$ generations meant that populations most likely experienced some genetic drift (Teitel et al. 2016a). However, as populations approached the fourth generation the effect of genetic drift was relatively negligible compared to other evolutionary processes like natural selection. My experimental design provided for detecting the consequences of genetic drift - if there was substantial genetic drift, there would be significant differentiation among populations, within experimental treatment combinations.

In 2014, wild and hybrid fruits were haphazardly collected from 30 plants from each of the 40 populations and stored in a labelled envelope. Two seeds from the first 15 plants, from each population, were collected and placed in labelled Eppendorf tubes $(1 \mathrm{~mL}$, Fischer Scientific ${ }^{\circ}$, Hampton, New Hampshire, U.S.A) and refrigerated at $4^{\circ}$ C. Two seeds from all 30 plants were collected from each CS population. Seeds collected from the 2014 populations were planted in the 2015 and 2016 experimental common gardens. Since wild radish has a long-lived seed bank, these seeds could have belonged to plants from the $\mathrm{F}_{2}$ to $\mathrm{F}_{4}$ generations; therefore, I refer to plants in 2015 and 2016 common gardens as $\mathrm{G}_{5}$ generation seeds.

\subsubsection{Rain-Out Shelters}

In the spring of 2015 (May 13-20) and 2016 (May 16-19), 30 rain-out shelters (2.44 m by $3.05 \mathrm{~m}$; Fig. 2.1) were constructed and erected in a randomized block design. In 2015, plots were established on a $20 \mathrm{~m} \times 18 \mathrm{~m}$ tilled plot of land and in 2016 plots were established on $25 \mathrm{~m} \times 25$ m tilled area approximately 210 meters southeast of my 2015 site (Fig. 2.1). My shelters were $3.05 \mathrm{~m}$ by $2.44 \mathrm{~m}$ wooden frames with transparent sheet plastic ( 3 mil, Canadian Greenhouse Suppliers, Niagara-on-the-Lake, Ontario, Canada) stretched over the frame, acting as a roof; new sheet plastic was applied each year (Fig. 2.2). Using metal poles, frames were slanted and elevated to approximately $1.2 \mathrm{~m}$ above ground at their lowest corner. Frames were slanted to 
intercept and divert natural rainfall into a $208 \mathrm{~L}$ plastic collection barrel via an eavestrough attached to the lowest side of the wooden frame. In 2015, shelters were arranged into five blocks, with each block's lowest corner angled (slanted) in a different direction to account for area limitations: block one slanted to the southwest, block two slanted to the southwest, block three slanted to the northeast, block four slanted to the northeast, and block five slanted to the northeast. In 2016, all shelter frames were slanted in the northeast direction, in the predominant direction of the rainfall.

\subsubsection{Common Garden Setup in 2015 and 2016}

In 2015 and 2016, I planted unique combinations (described below) of $\mathrm{G}_{5}$ hybrid and wild populations under 30 rain-out shelters. Each block consisted of three watering treatments DR, NR, and CS, replicated twice (for wild and hybrid populations), for a total of six plots per block. Water treatments applied in 2015 and 2016 will, hereafter, be referred to as current water treatments that plants were exposed to in each respective sheltered common garden. Under each shelter, soil was tilled and 50 and 48 pots in 2015 and 2016, respectively, were filled with local soil and buried flush with the ground on May $22^{\text {nd }}$ and $23^{\text {rd }}$ in 2015 and May $25^{\text {th }}$ to $27^{\text {th }}$ in 2016 (1 gallon black plastic pots, Bradford Co-Operative Storage, Ontario) in a $10 \times 6$ grid (2015) and $8 \times 6$ grid (2016) with pots approximately $30 \mathrm{~cm}$ apart. A single seed was planted approximately $1.3 \mathrm{~cm}$ below the soil surface of the pot and covered with soil. To minimize interspecific competition, weeds were removed on a weekly basis. A subset of historic wild and hybrid $\mathrm{G}_{5}$ water treatment seeds (i.e., seeds that have evolved under one of four selection histories described above) were randomly assigned to each current water treatment (Fig. 2.3). Additionally, in 2016, I planted a minimum of two Red Silk crop radish (R. sativus) seeds per plot, which were used in a separate study (Shukla et al., unpub). Historical seed combinations planted in the 2015 and 2016 current water treatments were as follows:

(1) Double Rain (DR) plots: I planted 15 wild and 15 hybrid individuals (i.e., three seeds from each of the five historical populations) that had historically evolved in DR conditions and ten wild and ten hybrid individuals that had historically evolved under CS conditions (two seeds per historical population) in the 2015 and 2016 current DR treatment plots. In 2016, a minimum of three additional crop seeds were planted per 
plot. In circumstances when sufficient number of replicates were not available per populations, I planted was all available seeds.

(2) No Rain (NR) plots: I planted 15 wild and 15 hybrid individuals that had historically evolved in NR conditions and ten wild and ten hybrid individuals that had historically evolved under CS conditions (two seeds per historical population) in the 2015 and 2016 current NR treatment plots. In 2016, a minimum of three additional crop seeds were planted per plot. In circumstances when sufficient number of replicates were not available per populations, I planted all available seeds.

(3) Control Rain (CS) plots: I planted 15 wild and 15 hybrid $\mathrm{G}_{5}$ individuals that had historically evolved in CU conditions and ten wild and ten hybrid $\mathrm{G}_{5}$ individuals that had historically evolved under CS conditions (two seeds per historical population) in the 2015 and 2016 current CS treatment plots. In 2016, a minimum of three additional crop seeds were planted per plot. In circumstances when sufficient number of replicates were not available per populations, I planted all available seeds.

Seeds were planted on three consecutive days in 2015 (May $26^{\text {th }}$ through $28^{\text {th }}$; approximately 1500 plants) and on two days in 2016 (May 30 ${ }^{\text {th }}$ and June $1^{\text {st. }}$; approximately 1400 plants). All pots were watered with approximately $100 \mathrm{~mL}$ of water once planted. Throughout the season, collected rainwater was applied manually to plots within 48-72 hours of a precipitation event; specific details associated with frequency and volume of water applied, see Appendix A-1. To minimize interspecific competition, weeds were removed on a weekly basis. Life-history measurements were made until flowering concluded and then plants were individually harvested until the experimental termination (October 14, 2015 and October 15, 2016), when all remaining plants were harvested. Harvested radish plants were dried for approximately one week at $65^{\circ} \mathrm{C}$ in a standing drying oven (VWR ${ }^{\circledR}$ ) in 2015 and air-dried in 2016 at the Koffler Scientific Reserve in King City, Ontario Canada and stored at Ryerson University.

\subsubsection{Phenology and Life History Measurements}

Each plant was monitored daily to record the date of seedling emergence and first flower during the experimental period from late May to October in 2015 and 2016. Time to seedling emergence was calculated as the difference between planting and date of emergence; Time to 
first flower was calculated as the difference between date of first flower and seedling emergence. Additionally, stem diameter, longest leaf length, and flower colour were measured on the date of first flower. Stem diameter and leaf length can be used as an indices of plant size and considered crop markers because they are heritable and highly correlated with crop ancestry (Campbell and Snow 2007; Campbell et al. 2009b; Warwick et al. 2009). Stem diameter was measured using digital calipers (Tresna Instruments ${ }^{\circledR}$, Guangxi Province, China) at the point of cotyledon attachment. Longest leaf length was assessed and measured using a standard Staples ${ }^{\circledR}$ (Massachusetts, USA) brand 30-cm ruler.

\subsubsection{Crop-Specific Trait Data Collection}

Flower colour is an easily identifiable crop trait marker that can be tracked through populations across time (Snow et al. 2001). Crop plants are characterized by their white flower colour which is a homozygous dominant phenotype, whereas wild plants have a yellow flower colour which is a homozygous recessive phenotype (Panetsos and Baker 1967). With white colour exhibiting simple Mendelian dominance over the yellow flower colour, the first generation of heterozygote hybrids will have exclusively white flowers, and the second generation of segregating hybrids is expected to express a 3 white: 1 yellow flower petal ratio. If flower colour is a selectively-neutral trait and is not correlated with other traits experiencing selection, this ratio should persist indefinitely, with the gene pool comprised of $50 \%$ crop alleles. The frequency of plants with white flower petal colour was recorded from the $\mathrm{F}_{1}$ to $\mathrm{G}_{5}$ generation. For $\mathrm{F}_{1}$ to $\mathrm{G}_{4}$, the frequency of plants with white flower petal colour was measured during peak flowering time ( June $25^{\text {th }}-$ July $\left.4^{\text {th }}, 2012-2014\right)$ and thus did not necessarily record flower petal colour of plants that flowered extremely early or late in the season. Estimates of flower colour frequency were based on direct counts when $<1000$ plants were present, or subsampling when populations were larger. For the latter, the average number of plants in $101-\mathrm{m}^{2}$ quadrats per site were determined and multiplied by the total area. Population sizes are available in Teitel et al. (2016c). These estimates were a single, instantaneous measurement of crop flower petal colour. However, in 2015 and 2016, flower petal colour was recorded for every plant that survived to flower, on the first day of flowering. 


\subsubsection{Chlorophyll Fluorescence}

To measure the photosynthetic performance of plants in the different watering treatments, and evaluate whether water stress or excess water influenced photosynthesis, I measured the dark-adapted quantum efficiency of photosystem II (PSII) as the ratio of variable $\left(\mathrm{F}_{\mathrm{v}}\right)$ to maximal $\left(F_{m}\right)$ chlorophyll fluorescence (Maxwell and Johnson 2000). Prior to experimental measurement, I randomly sampled 100 plants to determine a standard curve of the minimum time it took reaction centers to become fully oxidized; a dark adaptation period (period without light) where the photosynthetic pathway is free of electrons (i.e., the energy needed to drive photosynthesis) leaving reactions centers fully open ready to accept more electrons) (Goltsev et al. 2016). When reaction centers are fully oxidized, fluorescence remains consistent; this occurred after approximately 10 minutes for radish plants. Because all reaction centres were fully oxidized by shading prior to measurement, $\mathrm{F}_{\mathrm{v}} / \mathrm{F}_{\mathrm{m}}$ represents the maximum capacity of PSII to absorb light energy. In both years, I took outdoor measurements using a portable fluorescence meter (Handy PEA fluorometer, Hansatech Instruments Ltd., King's Lynn, Norfolk, UK). I non-destructively sampled quantum efficiency of PSII on a subset of twelve plants (three plants per historical environment $\times$ biotype combination) under each shelter for a total of 360 plants across the whole experiment in 2015 and another 360 plants in 2016. In random order, I measured plants after a 10 -minute dark adaptation period. Measurements in were collected on June $25^{\text {th }}$, July $18^{\text {th }}$, and August $10^{\text {th }}, 2015$ and between August 3 - 5, 2016. All measurements were taken between 8:00 $\mathrm{AM}$ and noon.

\subsubsection{Estimates of 2015 and 2016 Fecundity}

At the end of the 2015 growth year, a considerable number of plants ( 1200) survived to flower and produce seeds. In 2016, a substantially drier year, the number of plants that survived to flower decreased to $\sim 600$. Considering limits on time and resources, I wanted to determine if there was an allometric relationship between fitness (number of fruits and number of flower) and biomass. I randomly sampled six plants per historical treatment $\times$ biotype combinations per current treatment across five blocks, for a total of 180 plants (i.e., 6 plants per current treatment $\times 2$ biotypes $\times 3$ water treatments $\times 5$ blocks $=180$ ). The number of flowers and fruits were recorded, along with the above-ground biomass per plant minus any fruits, flower petals or leaves. To estimate number of flowers and fruits, the number of flower pedicels and thickened 
flower pedicels, respectively, were counted using tally counters. Additionally, a random sample of 10 fruits were collected and seeds were counted, recorded, and averaged to get an estimate of the number of seeds produced per fruit; these data, hereafter, are referred to as the allometric experiment. Next, I measured dry biomass on an additional subset ( $50 \%)$ of the remaining plants (void of fruits, flowers, and leaves) as a correlate of size and thus fecundity (Weiner et al. 2009); these data, hereafter, are referred to as the 50\% experiment. In 2016, a substantial portion of my harvested plants were eaten by rodents during storage and only 138 plants were assessed for the allometric experiment. The remaining plants ( 108) were used for the 50\% experiment. Analyses in section 2.3.10.3 and 2.3.10.4 were run with the data collected from the allometric experiment and 50\% experiment, combined. Traits analysed in these sections were days to seedling emergence, days to flowering, leaf length, stem diameter, and above-ground biomass.

\subsubsection{Statistical Analysis}

My experimental location moved between 2015 and 2016, therefore, I could not explicitly test for differences between years in most analyses. Instead, year was inherently accounted for within my block factor, such that blocks 1-5 represent 2015 data and blocks 6-10 represent 2016 data. Additionally, crop plants were omitted from analyses due to low replication between blocks (i.e., were only present in 2016). However, I performed a comparison for white flower colour frequency between generations (i.e., every year a new generation of plants were grown, except in 2015/2016 gardens) because I expected it to be insensitive to current environment context.

\subsubsection{Does the Frequency of Crop-Derived Alleles Vary Among Soil Moisture Treatments?}

If flower colour did not evolve, I expected the dominant white petal crop allele to persist at $50 \%$ frequency and thus $75 \%$ of the plants to have white flowers. To test whether the frequency of white flowers differed among hybrid populations across watering treatments over the four experimental generations $\left(\mathrm{G}_{2}\right.$ to $\left.\mathrm{G}_{5}\right)$, I ran an ANOVA where generation, watering treatment, and their interaction were fixed factors. Due to the white colour allele exhibiting dominance over the yellow colour allele, hybrid $\mathrm{F}_{1}$ flowers were all white $(100 \%$ of individuals

exhibit white flower petals). Therefore, the analysis included the $\mathrm{F}_{2}-\mathrm{G}_{5}$ generation (i.e., from 2012-2016), where 2015 and 2016 were combined into one generation (G5). Additionally, I ran a 
paired sample t-test to determine whether final white colour frequencies differed significantly from expected Hardy-Weinberg frequencies (75\%) across watering-histories. The analysis was run in SAS Enterprise (version 6.1, Cary, North Carolina, USA) using the PROC GLIMMIX function.

\subsubsection{Allometric Experiment-Can Biomass Be Used as a Tool to Assess Fitness?}

To determine the nature of the allometric relationship between biomass and fitness correlates (number of fruits and flowers and the number of seeds produced), I ran an ANOVA on the allometric experimental data only to test the relationship between number of flowers $(n=272)$, fruits $(n=267)$, and the number of seeds $(n=262)$ produced in response to genotype, historical watering treatment, their interaction, as fixed factors with biomass as a model covariate. Response variables were Box-Cox transformed, if necessary (Box Cox, Table 2.1), and analyses were run in R-Studio (lm and aov functions, R Core Team).

\subsubsection{Do Novel Environments Affect the Relative Success of Plants?}

To determine if current watering treatments (i.e., novel environments) had an effect on invading wild and hybrid plants, I ran a split-plot ANOVA on several measured traits in response to current watering treatment as the main plot effect, genotype as the split factor, and the interactions between current watering treatment and genotype as fixed factors with shelter (equivalent to block) as a random factor. Due to non-orthogonality of the data (i.e., not balanced), type III ANOVA results applied a Kenward-Roger's adjustment for computing the degrees of freedom (Luke 2017). Genotype, in these tests, refer to control shelter-evolved wild and hybrid plants, only. Current treatment refers to the watering treatment plants control shelterevolved plants were grown in (no rain, control, double rain). Traits tested from the combined allometric and 50\% experimental data include: life-history traits (seedling emergence times, flowering times, leaf length, and stem diameter, and quantum efficiency of photosystem II (PS II)) and the lifetime fecundity trait of biomass. All traits were Box-Cox transformed, if necessary. Split-plot ANOVA analyses were run in R-Studio (Table 2.2; Version 1.0.143; package lme4 and lmerTest) and SAS Enterprise Guide 61 (PROC GLM).

\subsubsection{Does Watering History Predispose Plants to Greater Success?}


Finally, to determine if historical watering treatments predisposed plants to greater success, I ran mixed model ANOVAs on combined trait data from the allometric and 50\% experiments in response to genotype, watering history treatment, their interaction as fixed factors, and block as a random factor. Due to non-orthogonality of the data (i.e., not balanced), type III ANOVA results applied a Kenward-Roger's adjustment for computing the degrees of freedom (Luke 2017). Traits tested include: life-history traits (seedling emergence times, flowering times, leaf length, stem diameter, and quantum efficiency of photosystem II (PS II)) and the lifetime fecundity trait of biomass. Analyses were done separately for each current watering treatment (i.e., NR - no rain, CS - control sheltered, DR - double rain plot data); genotype-by-historic watering treatment refer to wild and hybrid control-sheltered plants along with either wild or hybrid, no-rain, control-unsheltered, or double-rain plants, depending on the analysis. Analyses were run in R-Studio (version 1.0.143) and SAS Enterprise Guide 61 (PROC GLIMMIX) where all response variables were Box-Cox transformed, if necessary, and fitted to a normal distribution and an identity link function (Table 2.3). 


\subsection{Results}

\subsubsection{Evolution of Flower Colour in Hybrid Populations}

After the $F_{1}$ generation, colour composition of populations changed in a statistically significant manner through generations $\left(\mathrm{F}_{3,60}=3.70, \mathrm{p}<0.05\right.$, Fig. 2.4), with $\mathrm{G}_{2}$ having significantly lower white flower colour frequencies than $\mathrm{G}_{4}$ populations. The proportion of white flowered plants in $\mathrm{G}_{5}$ populations were 21\% less than the expected $75 \%$ Hardy-Weinberg frequency $\left(\mathrm{t}_{2,18}=-6.60, \mathrm{P}<0.01\right)$. However, white flower colour frequency in $\mathrm{G}_{5}$ populations did not vary with watering history or the interaction between watering history and year $\left(\mathrm{F}_{3,60}=0.16\right.$, $\mathrm{P}=0.92$ and $\mathrm{F}_{9,60}=0.63, \mathrm{P}=0.77$, respectively; Fig. 2.4).

\subsubsection{Can Biomass Be Used as a Correlate of Seed Set?}

Overall, wild plants produced fewer flowers $\left(\mu_{\text {wild }}=1435, \mu_{\text {hybrid }}=1456\right)$ and more fruits ( $\left.\mu_{\text {wild }}=848, \mu_{\text {hybrid }}=828\right)$ than hybrid plants but number of seeds per plant did not differ between genotypes (Table 2.1). Furthermore, the number of flowers, fruits, and seeds did not differ among watering histories or the interaction watering history had with genotype (Table 2.1).

As expected (Weiner et al. 2009), above-ground biomass was positively correlated with the number of flowers $\left(\mathrm{r}^{2}=0.71\right)$, fruits $\left(\mathrm{r}^{2}=0.64\right)$, and the total number of seeds $\left(\mathrm{r}^{2}=0.55\right)$ across plants (Table 2.1). Wild and hybrid plant biomass was also positively correlated with the number of fruits, flowers, and seeds but to varying degrees, depending on genotype (Table 2.1). Specifically, biomass was more strongly correlated with the number of flowers and fruits and total number of seeds in wild than hybrid populations $\left(\mathrm{r}^{2}\right.$ flower: Wild $(\mathrm{W})=0.59$, Hybrid $(\mathrm{H})$ $=0.50 ; \mathrm{r}^{2}$ fruit: $\mathrm{W}=0.59, \mathrm{H}=0.36 ; \mathrm{r}^{2}$ seeds per plant: $\left.\mathrm{W}=0.68, \mathrm{H}=0.50\right)$. Biomass significantly predicted the number of fruits, flowers, and number of seeds per plant across historical watering treatments (Table 2.1); for each trait: $\left[\mathrm{r}^{2}\right.$ flower: $\mathrm{NR}=0.73, \mathrm{CU}=0.71, \mathrm{CS}=0.68, \mathrm{DR}=0.82 ; \mathrm{r}_{\text {fruit: }} \mathrm{NR}=0.71$, $\mathrm{CU}=0.68, \mathrm{CS}=0.68, \mathrm{DR}=0.77 ; \mathrm{r}^{2}$ seeds per plant: $\left.\mathrm{NR}=0.73, \mathrm{CU}=0.62, \mathrm{CS}=0.51, \mathrm{DR}=0.67\right)$. Finally, biomass did not significantly differ in its ability to predict the number of flowers, fruits, or seeds among genotype-by-historic watering treatments. Since biomass was strongly correlated with the number of fruits, flowers, and seeds produced by all plants, I used these allometric relationships with biomass as a proxy of reproductive success in subsequent analyses that used data from the combined allometric and 50\% experimental data (sections 2.4.3 and 2.4.4) 


\subsubsection{Do Novel Environments Affect the Life-History and Relative Fecundity of Hybrid Plants?}

When historically control-sheltered hybrid plants invaded novel moisture environments, they emerged approximately one day earlier and flowered three days later than control-sheltered wild plants. Morphologically, control-sheltered hybrid plants had 24\% longer leaves, 35\% wider stem diameters, and had 34\% greater biomass than control-sheltered wild plants, irrespective of the environment they invaded. There was a marginally significant genotype (i.e., wild and hybrid plants from control sheltered histories) by current watering treatment interaction among plants with respect to emergence time (Table 2.2, Fig. 2.5) but pairwise differences were not significant. Current watering treatment by genotype interactions did not affect time to flower, leaf length, stem diameter, and above-ground biomass. Finally, the quantum efficiency of photosystem II did not differ between genotypes (CS wild and CS hybrid), current watering treatments (NR, CS, DR), or their interaction (Table 2.4).

\subsubsection{Does Watering History Predispose Hybrid Plants to Increased Fecundity Relative to Wild} Plants?

\subsubsection{No Rain Environments}

When grown in current no-rain environments, genotype (wild and hybrid; Fig. 2.6) and watering history (NR and CS plants; Fig. 2.7) significantly affected several life-history traits (Table 2.3). Days to flowering, leaf length, and stem diameter, but not days to seedling emergence, varied between genotypes such that hybrid plants flowered six days later, had 31\% longer leaves, and $18 \%$ wider stem diameters relative to wild plants. Despite these differences, plant biomass did not differ between hybrid and wild plants grown in current no-rain environments. Watering history had an effect on seedling emergence time but not days to flower, morphology (leaf length and stem diameter), or above-ground biomass. Specifically, no-rain plants emerged earlier (0.03 days; Fig. 2.7a) than CS-evolved plants when grown in current no rain environments. Furthermore, genotype-by-watering history treatment interactions did not affect days to seedling emergence, days to flower, morphology, or biomass (Table 2.3). Finally, the quantum efficiency of photosystem II did not differ between genotypes (wild and hybrid), watering histories (NR and CS), or their interaction (Table 2.4).

\subsubsection{Control Sheltered Environments}


When grown in current control-sheltered environments genotype (wild vs hybrid; Fig. 2.6) influenced days to flower and leaf length, and stem diameter but not days to emergence, or biomass (Table 2.3). Specifically, hybrid plants flowered approximately 4 days later and grew $19 \%$ wider stems than wild plants and had $7 \%$ marginally longer leaves. Watering history (CU and CS plants; Fig. 2.7) significantly affected plants such that control-unsheltered plants flowered approximately two days later than control-sheltered plants when grown in the same control sheltered watering environment (Table 2.3). When grown in current control-sheltered environments, seedling emergence times, leaf length, stem diameter and biomass did not significantly differ between genotypes (wild versus hybrid), watering history (CU versus CS plants), or their interaction (Table 2.3). Finally, the quantum efficiency of photosystem II did not differ between genotypes (wild and hybrid), watering histories (CU and CS), or their interaction (Table 2.4) when grown in current control-sheltered environments.

\subsubsection{Double Rain Environments}

When grown in current double-rain environments, genotype (wild and hybrid; Fig. 2.6) and watering history (DR and CS; Fig. 2.7), but not their interaction, affected plant growth (Table 2.3). Seedling emergence times statistically differed between genotypes grown in current double-rain environments, such that hybrid plants emerged earlier (0.3 days; Fig. 2.6a) than wild plants. Furthermore, hybrid plants relative to wild plants grown in current double-rain environments flowered five days later, had 34\% longer leaves and 62\% larger stem diameters, and had $87 \%$ more above-ground biomass. Watering history affected plants grown in current double rain environments, such that double-rain plants relative to control-sheltered plants emerged three days later, had $17 \%$ longer leaves and $25 \%$ larger stems, and were marginally $47 \%$ larger (Table 2.3, Fig. 2.8). Finally, the quantum efficiency of photosystem II (PS II) differed between genotypes (wild and hybrid) but not watering histories (DR and CS) or its interaction with genotype (Table 2.4). Specifically, quantum efficiency of PS II in hybrid plants was approximately $1.8 \%$ higher than the quantum efficiency of PS II in wild plants grown in current double-rain environments. 


\subsection{Discussion}

Abiotic environmental variation has been suggested as a mechanism that can affect gene flow which can alter opportunities for hybridization and influence the likelihood of crop alleles escaping into, and persisting, in wild populations (Arnold and Martin 2010; Arnold et al. 2013; Goulet et al. 2017; but see Campbell et al. 2016). Although the relative fitness of crop-wild hybrids has been studied in a range of environmental contexts, few have explicitly isolated forms of environmental variation and evaluate crop allele escape and persistence in hybrid-weed populations. My work is the first large-scale study attempting to quantify evolutionary changes in survival, fecundity, and crop-trait persistence of advanced-generation hybrids from variable environmental histories. My results reinforce that biomass can be an accurate predictor of plant reproductive success (i.e., number of flowers, number of fruits, and total seeds per plants), with the strength of this relationship increasing among watering environments with few differences between genotypes. In considering my first objective, when invading novel environments, hybridization can promote the evolution of earlier seedling emergence, delayed flowering, and larger plant size (leaf length, stem diameter, or biomass), relative to wild plants. After invading, larger plants with delayed flowering continued to persist in hybrid populations. Furthermore, hybrids in double rain conditions, evolved higher PSII functioning. Interestingly, wateringhistory only affected plants that evolved in double-rain environments which evolved later seedling emergence times but maintain delayed flowering and larger sizes than control-sheltered plants. Considering my third objective, despite differences in flowers and fruit numbers in the allometric experiment, hybridization and watering history did not affect the number of seeds per plant relative to wild plants across all current water treatments (NR, CU, DR). Finally, for my second objective, I detected parallel evolution of flower petal colour in hybrid populations across the four historical watering treatments (no rain, control-unsheltered, control-sheltered, and norain) over four generations $\left(\mathrm{G}_{2}-\mathrm{G}_{5}\right)$, suggesting that the influence of genetic drift on the introgression of this crop trait was minimal and that crop alleles persisted in consistent ways across populations and generations, regardless of soil moisture conditions. Considering this, I fail to reject the null hypothesis that water moisture does not affect white flower colour frequencies. Next, I discuss the success of crop-wild hybrids. Then, I look at the significance of a plant's growth environment in conjunction with its selection environment on weed success. Finally, I 
discuss how plant mating, particularly hybridization, may be influenced by environmental variation, with special consideration of global climate change scenarios.

\subsubsection{Persistence of Crop Traits in G5 Hybrids}

Since hybrid survival and reproductive success did not vary between watering environments, the persistence of crop-derived traits in wild radish populations appeared to be insensitive to the experimental range of watering conditions. Although there was yearly variation in crop allele frequencies, this variation did not reflect watering history but rather was due to differences in the way I measured flower colour frequencies. For instance, white flower colour was less frequent in the $\mathrm{G}_{3}$ and $\mathrm{G}_{4}$ generations when populations were sampled at a single time point, relatively early in the season. Since white flower colour is linked to late flowering (Campbell et al. 2009b), those generations may have been sampled too early to include late flowering plants with more crop-derived phenotypes (i.e., plants with delayed flowering may also be more likely to possess white flowers). In contrast, the $\mathrm{G}_{5}$ generation had complete sampling of all individuals and white flower colour frequency was higher across populations. Furthermore, considering white flower colour is correlated to flowering time (Campbell et al. 2009b), hybrid populations may be experiencing indirect selection against white flower colour due to direct selection against late flowering times (see below), therefore, white colour frequencies in the $\mathrm{G}_{5}$ generation did not meet Hardy-Weinberg expectations of selective neutrality.

Differences in the timing of seedling emergence and flowering in parents may provide subsequent segregating hybrid populations with the opportunity to optimize their phenology - an opportunity that does not appear to arise in either canonized parental population (Campbell et al. 2009b; Han et al. 2016). Although crops may possess relatively limited amounts of genetic diversity, the alleles they contributed may be entirely different from those of the recipient wild population and are often dominant genes of major effect (e.g., seedling emergence phenology; Nicotra et al . 2010). Consistent with previous generations (Teitel et al. 2016b), G5 hybrid seedlings emerged earlier when invading novel environments in my experimental gardens. Earlier seedling emergence, typical of crop plants, is a consequence of breeding efforts to reduce dormancy and synchronize yield (Gepts 2004), unlike wild plants which adaptively stagger germination throughout the season to avoid total failure due to stressful or unfavourable growth 
conditions (Gepts 2004; Anderson et al. 2011). My results are consistent with others where cropwild hybrid populations (e.g., Helianthus species) have evolved crop-like emergence times and/or reduced dormancy (Mercer et al. 2007; Mercer et al. 2014). Hybrid radishes, in general, flowered later, produced more flowers, but not more fruits, compared to wild radish plants. Flowering duration and number of flowers can be an important component to the success and potential weediness of a plant (Amasino and Michaels 2010), and a correlate of male fitness, where more flowers and continued flowering through the growth season can increase the probability of successful pollination- a wild-derived trait (Franks et al. 2007; Hoffmann and Sgrò 2011; Ellstrand et al. 2013). Although hybrid plants were not more fecund than wild plants, their average seed production was equivalent to that of wild plants. In contrast, in $\mathrm{G}_{4}$ Michigan populations, Campbell et al. (2006) found that hybrids produced more flowers than wild plants but were $11 \%$ less fecund. My results, along with Campbell et al. (2006), suggest that advancedgeneration hybrid plants can retain crop-derived traits to produce adaptive phenotypes across water histories.

\subsubsection{Allometric Models: Relative Importance of Hybridization and Selection on the Success of Invasiveness in Raphanus Species}

To measure the relative fitness of my wild and crop-wild hybrid plants, I needed to collect data of the number of flowers and fruits and total fecundity of each plant produced. However, on a sample size of more than 2900 plants, this data collection is very time consuming. Using allometric models, I hoped to find a model using a proxy variable that would allow me to predict the number of flowers and fruits and total seeds per plant, relatively quickly. Plant reproductive success is often positively correlated with plant biomass (Younginger et al. 2017) where short-lived herbaceous plants like radish, for example, can show a linear relationship between biomass and reproduction (i.e., number of seeds per plant) or reproductive correlates (i.e., number of fruits and flowers; Weiner et al. 2009; Younginger et al. 2017). I found that plant biomass, across all plants from all watering histories, confidently predicted the number of flowers and fruits and total number of seeds. Among genotypes, wild above-ground biomass was a stronger predictor of flowers and fruits than hybrid above-ground biomass. Wild and hybrid biomass, however, could not significantly predict the total seeds produced. Previous work in radish populations has shown that wild radish plants had stronger positive correlations between the number of flowers produced and seeds per fruit relative to hybrid plants (Campbell and Snow 
2007). However, once wild and hybrid plants were exposed to competition (i.e., a selection pressure), seeds per fruit decreased in wild plants and diminished the overall difference between genotypes (Campbell and Snow 2007). Furthermore, biomass did not significantly differ in its ability to predict the number of flowers, fruits, or seeds among genotype-by-historic watering treatments. Interestingly, biomass of plants from extreme moisture environments was a far better, stronger predictor of the number of flowers, fruits, and total seeds per plant. The data from my work suggests that biomass in general is a valid tool to predict invasive success (measured via the number of flowers and fruits and total seeds produced) - this result parallels other work (Weiner et al. 2009; Younginger et al. 2017). A novel finding, however, is that biomass of plants from different selection history (i.e., watering history in this experiment) rather than hybridization, is a stronger and more effective predictor of reproductive success in my populations.

\subsubsection{Environmental Context: An Important Determinant of Invasive Success in Radish Hybrid Populations}

The success of crop-wild Raphanus hybrid populations has been geographically variable across North America (Campbell et al. 2006; Campbell and Snow 2007; Ridley and Ellstrand 2010; Hovick et al. 2012). Californian crop-wild hybrid radishes have, undoubtedly, been the most successful. Crop-wild hybrid radish populations (referred to as wild radish or California wild radish, $R$. sativus or $R$. raphanistrum $\times R$. sativus) have replaced all wild radish (jointed charlock, $R$. raphanistrum) populations in California (Hegde et al. 2006); they are more likely to survive to reproduce, and produce more flowers and seeds than $R$. raphanistrum populations (Campbell et al. 2006). Hybrid radishes have been extremely successful invaders when introduced into novel regions, as well. In Texas, hybrid radish displayed greater seedling and flowering frequency and earlier emergence times that translated into three times more seed production than wild plants (Hovick et al. 2012). However, crop-wild radish populations are not always more successful relative to wild radish progenitors. Hybrid populations in Michigan (a non-novel environment), in comparison, are successful competitors at par with wild populations (Campbell \& Snow 2007). Early-generation Michigan hybrid populations displayed lower fertility and fitness than wild progenitors (Snow et al. 2001) but have been shown to quickly regain fertility, fruit and flower production, and seed set less than or equivalent to $R$. raphanistrum populations (Campbell et al. 2006). Evolution of early-generation hybrid 
populations has resulted in decreased dormancy periods, increased germination frequency, earlier emergence, and earlier flowering (Campbell et al. 2006; Campbell and Snow 2007; Campbell et al. 2009b; Teitel et al. 2016c). Ontario crop-wild populations, despite displaying phenological and fecundity-enhancing traits (i.e., flower production), did not outcompete wild populations but remain relatively equivalent. Furthermore, quantum efficiency of PSII (i.e., a proxy measuring photosynthetic efficiency) did not vary between genotype and invading moisture environment and remained relatively constant $(\sim 0.78 \pm 0.08$ std. dev.). This suggest that invading new soil moisture environments may not prove to be stressful and a strong determinate of the relative success of hybrid to wild radish plants. An important distinction, however, lies in the selection pressures that populations are responding to, such as varying density and competition pressures, suggesting watering history, as the only selection pressure, may not determine hybrid success.

\subsubsection{Environmental Selection History Can Affect Plant Survival and Fecundity}

Plant survival and reproduction requires that each plant survives to germinate, grow, flower and produce viable seeds. After five generations of experiencing a moisture cline hybrid radish plants that evolved in NR, CU, CS, and DR selection histories flowered later, had similar emergence times, and number of seeds per plant relative to wild plants from the same selection histories. Long-term drought may impede population establishment and survival (Teitel et al. 2016c) but plants that survive establishment produce as many seeds as plants from moisture-rich environments, as my data demonstrate. Maternal environments can influence the way mother plants provision their offspring and indirectly influence success of offspring and grand-offspring (Campbell et al. 2015; Metz et al. 2015; Walter et al. 2016). Wild radish (R. raphanistrum) offspring and grand-offspring from NR conditions were substantially smaller (seed biomass, floral display, and plant size), in contrast, to seeds and plants from DR maternal environments (Campbell et al. 2015). Additionally, my data show that water-evolved (NR, CU, DR) hybrids were consistently larger than water-evolved wild plants. Moisture conditions can affect morphological traits associated with size (e.g., stem or biomass) or floral indicators can affect plant success. For example, wild radish populations that evolved in DR conditions (Campbell et al. 2015), were larger than plants from NR environments and larger plant size can influence competitive ability (Campbell and Snow 2007). Along with increased biomass, corolla diameter and anther length increased in plant floral display and aid in pollinator-attractions and 
fertilization success (Pirimova et al. 2015; Sapir et al. 2017). The range of drought experienced by plants in my experiments may not influence relative fecundity of plants but successful distribution of weedy radish populations may be dictated by other abiotic selection pressures.

Water and other abiotic selection factors have been shown to have a substantial effect on performance and distribution in other plant species. In New Caladonia - an island system in the south Pacific - changing microenvironments, mostly mediated by temperature and precipitation changes, have created the opportunity for increased gene flow and hybridization between three Coffea species (Berecha et al. 2014). Increased gene flow created opportunity for new hybrid zones (as acquired from all three Coffea species) in areas which they (crop plants and alleles) were not previously inhabiting (Berecha et al. 2014). Hybrid Ipomosis populations, when exposed to drought conditions had higher water-use efficiency and flower production than parental populations in the same environment (Wu and Campbell 2006; Campbell and Waser 2007; Campbell et al. 2010; Campbell and Wendlandt 2013). Although there was no difference in the response of wild and hybrid populations to a soil moisture gradient, water can be a strong selective force in promoting and maintaining more successful hybrid populations relative to wild progenitors.

Species distributions may be better explained when incorporating multiple abiotic and biotic selection factors rather than a single abiotic factor; creating scenarios representative of the environments experienced by natural populations. For example, to determine how competitive ability was affected by maternal moisture availability, populations of Biscutella didyma were grown in two contrasting moisture environments (abundant water and drought) with and without competitors. Researchers found coming from resource rich environments had higher competitive advantages with higher survival rates, greater biomass, and were more fecund than plants from drought environments (Metz et al. 2015) but did not differ when competitors were not present. Competitive selection pressures have also been studied in the Sonoran Desert plant Dithyera californica, where seed width is known to be positively correlated with competitive strength. However, when D. californica is grown in high plant densities, they selectively produce smaller seeds that can disperse farther than larger seeds (Metz et al. 2015). Although smaller seeds have a higher probability of survival, there still presents a negative trade-off with competitive ability in new environments exposed to new competitors (Larios and Venable 2015). Following these examples, to understand the continued evolutionary success of weedy populations, and whether 
hybridization will facilitate weedy success, large scale experimentation is still needed using multiple abiotic and biotic selection pressures.

\subsubsection{The Influence of Climate on Hybridization and Mating Systems}

Water availability may feature as an important environmental influence on the opportunities for plants to self-fertilize versus outcross pollinate and can thus strongly influence plant investment in various reproductive strategies (Fischer and Turner 1978; Abdel-Ghani et al. 2004; Flexas et al. 2004; Kannadan and Rudgers 2008), which may vary dramatically across a growing season (Adamowski et al. 2013; Taxak et al. 2014), and exhibit significant spatial variation (refer to Fig 1.1 in Chapter 1). Shifts in mating strategies are a consequence of the response to climatic variation of underlying population characteristics, such as plant floral display, flower size, colour, or sex ratio of populations, and/or the ratio of open to closed flowers (Barrett 2014). In the mixed mating plant, Viola praemorsa, there was a $61 \%$ increase in selfing, cleistogamous (closed) flowers and $45 \%$ of potentially outcrossing, chasmogamous (open) flowers reverted to selfing strategies with increased temperature and drought conditions (Jones et al. 2013). Plants can also experience a breakdown of self-incompatibility genetic mechanisms that permit selfing when exposed to extreme high temperatures or drought (Levin 2010). These underlying biological processes, in general, can influence the degree of outcrossing within populations and in response to extreme environmental shifts, the proportion of selfing can drastically increase and affect the genetic diversity of a population. Little empirical evidence exists to test these theoretical predictions in the context of hybridization - with my experiment being some of the first preliminary work demonstrating that soil moisture creates a geographic mosaic of selection. In the broader context of climate change, traits that may distinguish mating system selection (selfing to outcrossing) and the frequency of hybridization, like floral morphology or pollinator availability (Goodwillie et al. 2005; Eckert et al. 2009; Van Etten and Brunet 2013), it will be imperative especially population establishment where either the source population or incipient population is located in drought-stricken areas. 


\subsection{Table List}

Table 2.1: To determine if biomass could be used a proxy to estimate fitness, I ran a model testing the allometric relationship between biomass and the a) number of flowers, b) number of fruits, and c) number of seeds produced. Response variables were tested in response to Genotype (G: wild - W and hybrid - H), historical watering treatment (WH: no rain - NR, control unsheltered - CU, control sheltered - CS, double rain - DR), and the covariate of biomass (Bi), and their interactions therein. Correlation values $\left(\mathrm{r}^{2}\right)$ are presented for models where biomass significantly correlated with a factor (genotype and watering history). Analyses were run in RStudio (version 1.0.143). F-statistics are presented to indicate significant differences: $\mathrm{ns}, \mathrm{P}>$ $0.10 ;+, \mathrm{P}<0.10 ; *, \mathrm{P}<0.05 ; * *, \mathrm{P}<0.01 ; * * *, \mathrm{P}<0.001$.

\begin{tabular}{|c|c|c|c|}
\hline Responses \& Parameters & df $(n, d)$ & F-value & Correlation Value $\left(\mathbf{r}^{2}\right)$ \\
\hline \multicolumn{4}{|c|}{ a) Number of Flower $(n=272 ;$ Box-Cox transformation, $\lambda=0.1534275)$} \\
\hline Biomass $(\mathrm{Bi})\left(\mathrm{r}^{2}=0.71\right)$ & 1,256 & $301.75^{* * *}$ & \multirow{7}{*}{$\begin{array}{c}\text { W:0.59, H:0.50 } \\
\text { NR:0.73, CU:0.71, } \\
\text { CS:0.68, DR:0.82 }\end{array}$} \\
\hline Genotype $(\mathrm{G})$ & 1,256 & $6.39^{*}$ & \\
\hline Watering History $(\mathrm{WH})$ & 3,256 & $0.45^{\mathrm{ns}}$ & \\
\hline $\mathrm{Bi} \times \mathrm{G}$ & 1,256 & $16.01^{* * *}$ & \\
\hline $\mathrm{Bi} \times \mathrm{WH}$ & 3,256 & $2.73 *$ & \\
\hline $\mathrm{G} \times \mathrm{WH}$ & 3,256 & $1.18^{\mathrm{ns}}$ & \\
\hline $\mathrm{Bi} \times \mathrm{G} \times \mathrm{WH}$ & 3,256 & $0.73^{\mathrm{ns}}$ & \\
\hline \multicolumn{4}{|c|}{ b) Number of Fruits $(n=267$; Box-Cox transformation, $\lambda=0.2342124)$} \\
\hline $\mathrm{Bi}$ & 1,252 & $211.04^{* * * *}$ & \multirow{7}{*}{$\begin{array}{c}\text { W:0.59, H:0.36 } \\
\text { NR:0.71, CU:0.68, } \\
\text { CS:0.68, DR:0.77 }\end{array}$} \\
\hline G & 1,252 & $8.61^{* *}$ & \\
\hline WH & 3,252 & $1.35^{\mathrm{ns}}$ & \\
\hline $\mathrm{Bi} \times \mathrm{G}$ & 1,252 & $19.62^{* * *}$ & \\
\hline $\mathrm{Bi} \times \mathrm{WH}$ & 3,252 & $3.19^{*}$ & \\
\hline $\mathrm{G} \times \mathrm{WH}$ & 3,252 & $1.08^{\mathrm{ns}}$ & \\
\hline $\mathrm{Bi} \times \mathrm{G} \times \mathrm{WH}$ & 3,252 & $0.63^{\mathrm{ns}}$ & \\
\hline \multicolumn{4}{|c|}{ c) Number of Seeds $(n=262 ;$ Box-Cox transformation, $\lambda=0.2586738)$} \\
\hline $\mathrm{Bi}$ & 1,246 & $127.64^{* * *}$ & \multirow{7}{*}{$\begin{array}{c}\text { W:0.68, H:0.50 } \\
\text { NR:0.73, CU:0.62, } \\
\text { CS:0.51, DR:0.67 }\end{array}$} \\
\hline G & 1,246 & $2.09^{\mathrm{ns}}$ & \\
\hline WH & 3,246 & $1.99^{\mathrm{ns}}$ & \\
\hline $\mathrm{Bi} \times \mathrm{G}$ & 1,246 & $22.99^{* * *}$ & \\
\hline $\mathrm{Bi} \times \mathrm{WH}$ & 3,246 & $3.19^{*}$ & \\
\hline $\mathrm{G} \times \mathrm{WH}$ & 3,246 & $1.40^{\mathrm{ns}}$ & \\
\hline $\mathrm{Bi} \times \mathrm{G} \times \mathrm{WH}$ & 3,246 & $0.58^{\mathrm{ns}}$ & \\
\hline
\end{tabular}


Table 2.2: To estimate whether hybrid plants that invade novel environments perform better relative to wild plants, I ran a split-plot mixed model ANOVA on (a - d) several life history traits and (e) a single lifetime fecundity trait in response fixed effects current water treatment (CT: no rain-NR, control shelter-CS, double rain- DR), and genotype (G: wild, hybrid) as the split-plot factor, and their interactions, with shelter (S: 10 levels) as a random factor. Analyses were done on data collected from CS-evolved wild and hybrid plants that grew in NR, CS, and DR soil moisture environments. Across models, CT was tested against the whole plot error interaction [i.e., between subject's error; presented as $\operatorname{Error}(S \times C T)$ in the table] while the within subject's effects $(\mathrm{G}$ and $\mathrm{G} \times \mathrm{CT})$ are tested against the residuals error terms (presented as Error in the table). Additionally, considering the non-orthogonality of the data, type III ANOVA results are presented in the table using a Kenward-Roger's adjustment for computing the degrees of freedom. Split-plot analyses were run in R-Studio (version 1.0.143) and SAS Enterprise Guide 61 (PROC GLM). BoxCox transformations $(\lambda)$ and sample sizes (n) are reported for each response variable. F-statistics in the fixed effect ANOVA table are presented to indicate significant differences: $\mathrm{ns}, \mathrm{P}>0.10 ;+$, $\mathrm{P}<0.10 ; *, \mathrm{P}<0.05 ; * *, \mathrm{P}<0.01 ; * * *, \mathrm{P}<0.001$.

\begin{tabular}{|c|c|c|c|}
\hline $\begin{array}{l}\text { Response \& } \\
\text { Parameter }\end{array}$ & dff & Mean Square & F Statistic \\
\hline \multicolumn{4}{|c|}{ a) Days to Emergence (Box-Cox transformation $\lambda=-1.125741, \mathrm{n}=206$ ) } \\
\hline Shelter & 9 & $5.79 \times 10^{-3}$ & $9.96^{* * *}$ \\
\hline Current Treatment (CT & 2 & $7.38 \times 10^{-4}$ & $1.27^{\mathrm{ns}}$ \\
\hline $\operatorname{Error}(S \times C T)$ & 17 & $5.81 \times 10^{-4}$ & - \\
\hline Genotype (G) & 1 & $1.34 \times 10^{-3}$ & $3.69^{*}$ \\
\hline $\mathrm{CT} \times \mathrm{G}$ & 2 & $9.23 \times 10^{-4}$ & $2.53^{+}$ \\
\hline Error & 174 & $2.64 \times 10^{-4}$ & - \\
\hline \multicolumn{4}{|c|}{ b) Days to Flowering (Box-Cox transformation $\lambda=-2.475887, \mathrm{n}=206$ ) } \\
\hline $\mathrm{S}$ & 9 & $1.16 \times 10^{-9}$ & $1.42^{\mathrm{ns}}$ \\
\hline $\mathrm{CT}$ & 2 & $6.01 \times 10^{-10}$ & $0.73^{\mathrm{ns}}$ \\
\hline $\operatorname{Error}(S \times C T)$ & 17 & $8.17 \times 10^{-10}$ & - \\
\hline G & 1 & $6.63 \times 10^{-9}$ & $12.81^{* * *}$ \\
\hline $\mathrm{CT} \times \mathrm{G}$ & 2 & $5.94 \times 10^{-10}$ & $1.15^{\mathrm{ns}}$ \\
\hline Error & 174 & $5.18 \times 10^{-10}$ & - \\
\hline \multicolumn{4}{|c|}{ c) Longest Leaf Length (Box-Cox transformation $\lambda=0.3485819, \mathrm{n}=206$ ) } \\
\hline $\mathrm{S}$ & 9 & 6.73 & $5.85^{* * *}$ \\
\hline CT & 2 & 0.67 & $0.58^{\mathrm{ns}}$ \\
\hline $\operatorname{Error}(S \times C T)$ & 17 & 1.15 & - \\
\hline $\mathrm{G}$ & 1 & 10.64 & $13.80^{* * *}$ \\
\hline $\mathrm{CT} \times \mathrm{G}$ & 2 & 0.19 & $0.25^{\mathrm{ns}}$ \\
\hline Error & 174 & 0.77 & - \\
\hline \multicolumn{4}{|c|}{ d) Stem Diameter (Box-Cox transformation $\lambda=0.06130056, \mathrm{n}=206)$} \\
\hline S & 9 & 1.93 & $8.04^{* * * *}$ \\
\hline $\mathrm{CT}$ & 2 & 0.31 & $1.27^{\mathrm{ns}}$ \\
\hline $\operatorname{Error}(S \times C T)$ & 17 & 0.24 & - \\
\hline $\mathrm{G}$ & 1 & 3.55 & $13.12^{* *}$ \\
\hline $\mathrm{CT} \times \mathrm{G}$ & 2 & 0.17 & $0.65^{\mathrm{ns}}$ \\
\hline Error & 174 & 0.27 & - \\
\hline
\end{tabular}




\begin{tabular}{lccc}
\hline e) Above-ground Biomass (Box-Cox transformation $\lambda=0.2624348, \mathrm{n}=206)$ & \\
\hline $\mathrm{S}$ & 9 & 31.06 & $4.84^{* *}$ \\
$\mathrm{CT}$ & 2 & 5.00 & $0.78^{\mathrm{ns}}$ \\
Error $(S \times C T)$ & 17 & 6.41 & - \\
$\mathrm{G}$ & 1 & 17.70 & $4.03^{*}$ \\
$\mathrm{CT} \times \mathrm{G}$ & 2 & 0.63 & $0.14^{\mathrm{ns}}$ \\
Error & 174 & 4.40 & - \\
\hline
\end{tabular}


Table 2.3: To estimate whether watering history pre-disposes hybrid plants to perform better than wild plants, I ran a mixed model ANOVA on (a - d) several life history traits and (e) a single lifetime fecundity trait for each current watering environment (no rain- NR, control shelter-CS, and double rain-DR). For each watering environment, I ran traits in response to watering history (WH: NR plots: NR and CS; CS plots: control unsheltered and CS; DR plots: DR and CS) and genotype (wild and hybrid) as the fixed factors and block (10 levels) as the random factor. Considering the non-orthogonality of the data, type III ANOVA results are presented in the table using a Kenward-Roger's adjustment for computing the degrees of freedom. The fixed effects models compute the F-statistic using the mean square error of each model (presented as Error in the table). Traits that were analysed included a) days to seedling emergence, $b$ ) days to flower, c) leaf length, d) stem diameter, and e) above-ground biomass in each current watering environment. Additionally, each trait also lists the $\chi^{2}$ significance, and associated degrees of freedom, comparing the model with and without the block factor (i.e, measuring the significance of block in the model). Analyses were run in R-Studio (version 1.0.143) and SAS Enterprise Guide 61 where all response variables were fitted to a normal distribution and an identity link function. Box-Cox transformations $(\lambda)$ and sample sizes $(n)$ are reported for each response variable. F-statistics in the fixed effect ANOVA table are presented to indicate significant differences: $\mathrm{ns}, \mathrm{P}>0.10 ;+, \mathrm{P}<0.10 ; *, \mathrm{P}<0.05 ; * *, \mathrm{P}<0.01 ; * * *, \mathrm{P}<$ 0.001 . 


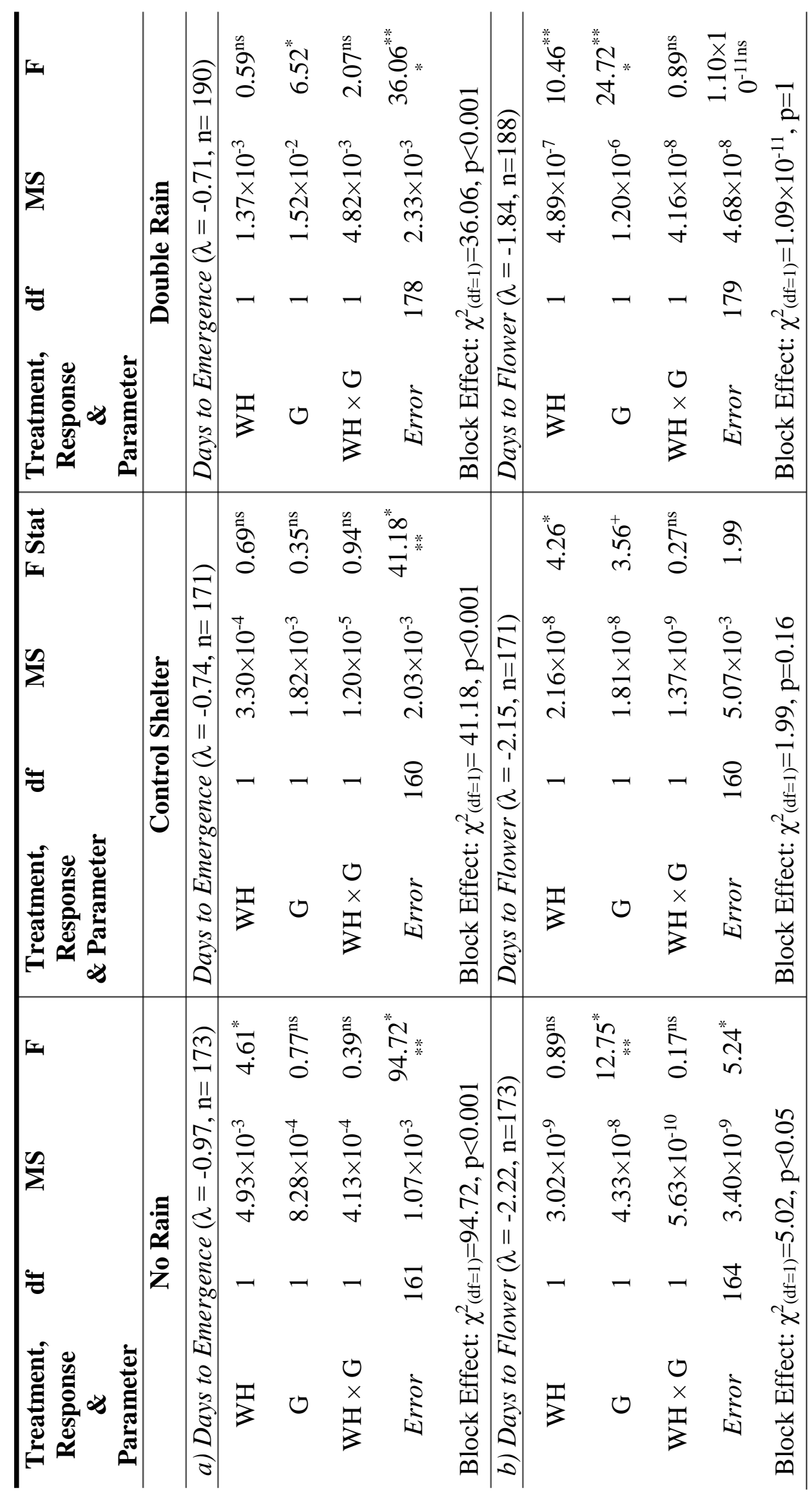




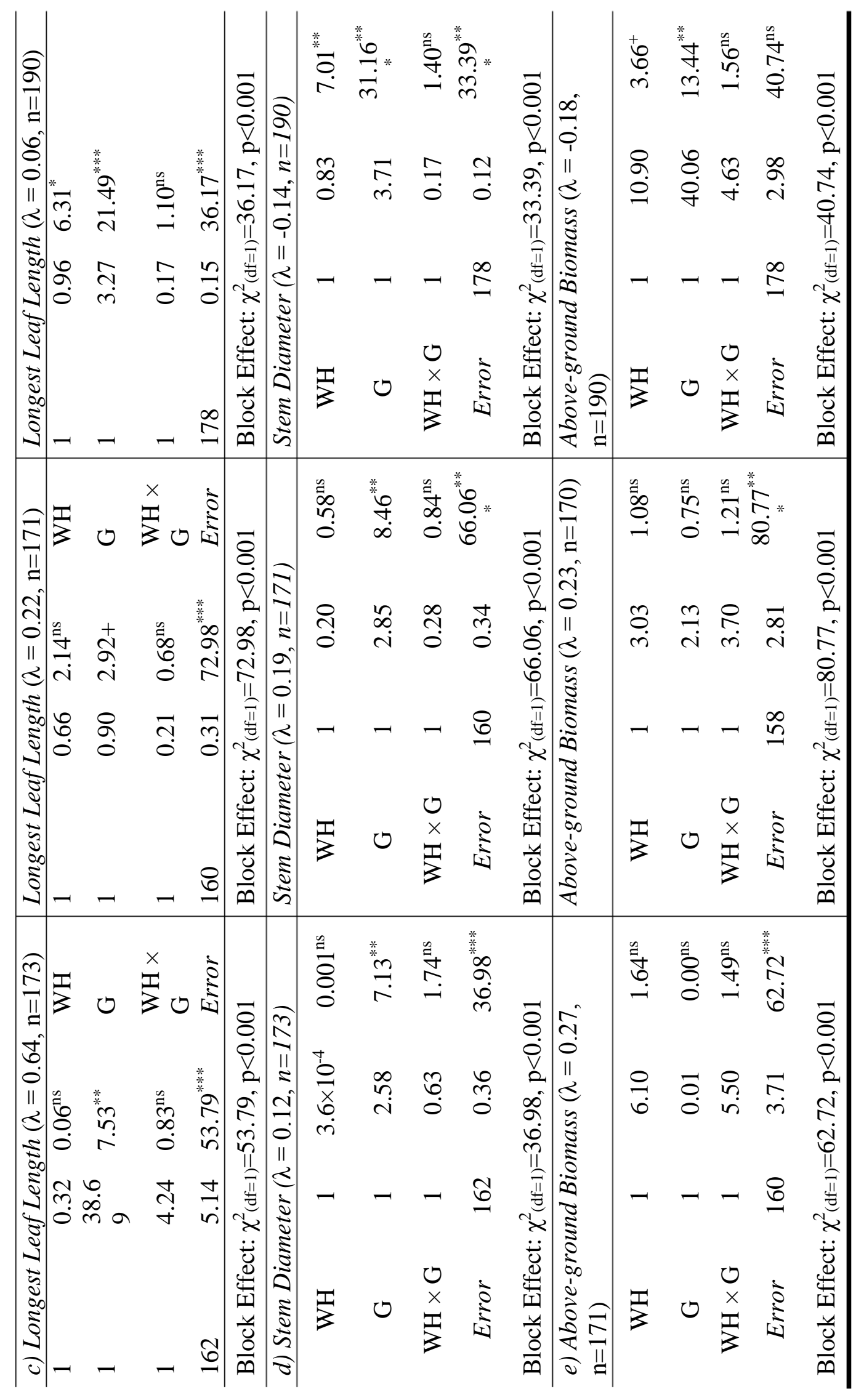


Table 2.4: To evaluate whether water stress influenced photosynthesis, I compared the darkadapted quantum efficiency of photosystem II (PSII) across wild and hybrid CS plants grown in all current watering treatments. (a) To estimate whether hybrid plants (with a shared control sheltered watering history) that invaded novel environments (current watering treatments [CT]: no rain-NR, control shelter-CS, double rain- DR) have higher quantum efficiency relative to wild plants, I ran a split-plot mixed model ANOVA on chlorophyll fluorescence in response to current water treatment (CT:), genotype (wild, hybrid) as the split factor, and interactions therein as the fixed factors and Shelter (S: 10 levels) as a random factor. Within the model, CT was tested against the whole plot error interaction [i.e., between subject's error; presented as Error $(S \times C T)$ in the table] while the within subject's effects $(\mathrm{G}$ and $\mathrm{G} \times \mathrm{CT})$ were tested against the residuals error terms (presented as Error in the table). Next, to estimate whether watering history predisposes hybrid plants to have higher PSII efficiency than wild plants, a mixed model ANOVA was run on plants in current watering treatments of (b) no rain plots, (c) control sheltered plots, and (d) double rain plots, respectively, on chlorophyll fluorescence values in response to genotype (G: wild and hybrid) and watering history (in current NR plots: NR and CS WH; current CS plots: control unsheltered and CS WH; and current DR plots: DR and CS WH), and their interactions as fixed factors and block as the random factor. Additionally, models $b-\mathrm{d}$ also list the $\chi^{2}$ significance, and associated degrees of freedom, comparing the model with and without the block factor (i.e, measuring the significance of block in the model). Considering the non-orthogonality of the data, type III ANOVA results are presented in the table using a Kenward-Roger's adjustment for computing the degrees of freedom. Analyses were run in RStudio (version 1.0.143) and SAS Enterprise Guide 61 (PROC GLM) where all response variables were fitted to a normal distribution and an identity link function. Box-Cox transformations $(\lambda)$ and sample sizes $(n)$ are reported for each response variable. F-statistics in the fixed effect ANOVA table are presented to indicate significant differences: $\mathrm{ns}, \mathrm{P}>0.10 ;+, \mathrm{P}$ $<0.10 ; *, \mathrm{P}<0.05 ; * *, \mathrm{P}<0.01 ; * * *, \mathrm{P}<0.001$.
Response \& df Mean Square F Statistic Parameter

\begin{tabular}{|c|c|c|c|}
\hline \multicolumn{4}{|c|}{ a) Control Shelter Plants (Box-Cox transformation $\lambda=8.309729, n=286$ ) } \\
\hline Shelter & 9 & $5.44 \times 10^{-5}$ & $0.90^{\mathrm{ns}}$ \\
\hline $\begin{array}{l}\text { Current Treatment } \\
\text { (CT) }\end{array}$ & 2 & $6.46 \times 10^{-5}$ & $1.07^{\mathrm{ns}}$ \\
\hline Error $(S \times C T)$ & 18 & $6.03 \times 10^{-5}$ & - \\
\hline Genotype (G) & 1 & $3.60 \times 10^{-5}$ & $0.87^{\mathrm{ns}}$ \\
\hline $\mathrm{CT} \times \mathrm{G}$ & 2 & $8.13 \times 10^{-6}$ & $0.20^{\mathrm{ns}}$ \\
\hline Error & 253 & $4.12 \times 10^{-5}$ & - \\
\hline \multicolumn{4}{|c|}{ b) No Rain Plots (Box-Cox transformation $\lambda=7.431859, \mathrm{n}=161$ ) } \\
\hline $\begin{array}{l}\text { Watering History } \\
\text { (WH) }\end{array}$ & 1 & $7.33 \times 10^{-6}$ & $0.10^{\mathrm{ns}}$ \\
\hline $\mathrm{G}$ & 1 & $1.41 \times 10^{-5}$ & $0.19^{\text {ns }}$ \\
\hline $\mathrm{WH} \times \mathrm{G}$ & 1 & $1.11 \times 10^{-5}$ & $0.15^{\mathrm{ns}}$ \\
\hline Error & 149 & $7.36 \times 10^{-5}$ & - \\
\hline \multicolumn{4}{|c|}{ Block Effect $: \chi_{(\mathrm{df}=1)}^{2}=3.99, \mathrm{p}<0.05$} \\
\hline \multicolumn{4}{|c|}{ c) Control Shelter Plots (Box-Cox transformation $\lambda=9.47536, \mathrm{n}=206$ ) } \\
\hline WH & 1 & $3.78 \times 10^{-6}$ & $0.19^{\mathrm{ns}}$ \\
\hline G & 1 & $1.69 \times 10^{-6}$ & $0.08^{\text {ns }}$ \\
\hline
\end{tabular}



$\mathrm{WH} \times \mathrm{G}$
1
$3.22 \times 10^{-8}$
$1.60 \times 10^{-3 \mathrm{~ns}}$
Error
194
$2.00 \times 10^{-5}$

Block Effect: $\chi_{(\mathrm{df}=1)}^{2}=0.57, \mathrm{p}=0.45$

\begin{tabular}{lccc}
\hline d) Double Rain Plots (Box-Cox transformation $\lambda=0.06130056, \mathrm{n}=212)$ & \\
\hline $\mathrm{WH}$ & 1 & $2.39 \times 10^{-5}$ & $0.83^{\mathrm{ns}}$ \\
$\mathrm{G}$ & 1 & $2.01 \times 10^{-4}$ & $7.05^{*}$ \\
$\mathrm{WH} \times \mathrm{G}$ & 1 & $6.39 \times 10^{-5}$ & $2.23^{\text {ns }}$ \\
Error & 199 & $2.86 \times 10^{-5}$ & - \\
Block Effect: $\chi^{2}(\mathrm{df}=1)=1,10, \mathrm{p}=0.29$ & & \\
\hline
\end{tabular}




\subsection{Figure List}
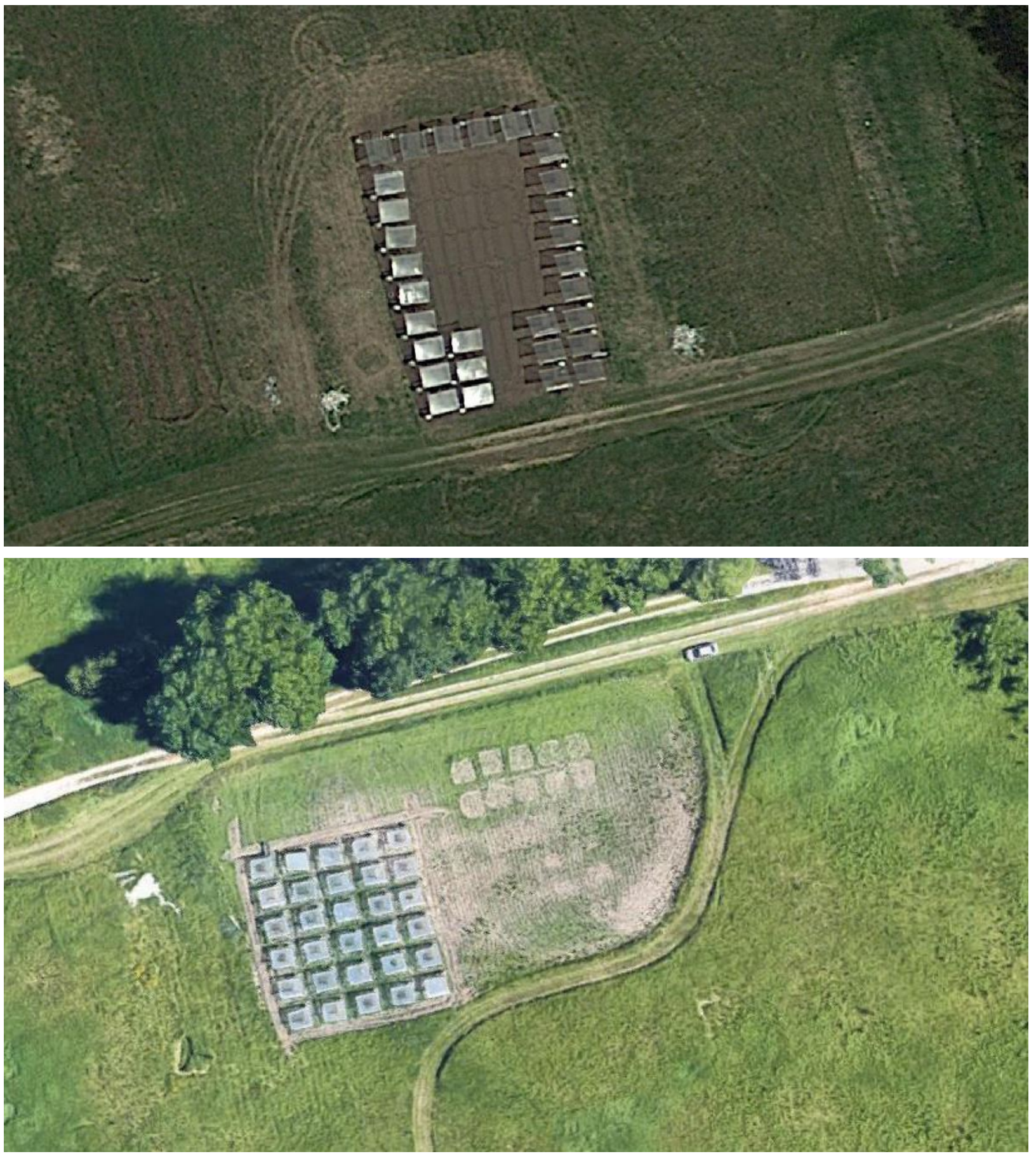

Figure 2.1: Experimental area of 2015 (above) and 2016 (below) shelter common garden experiments at the Koffler Scientific Reserve. King City, Ontario. Thirty rain-out shelters (2.44 $\mathrm{m}$ by $3.05 \mathrm{~m}$ ) were constructed and erected in a randomized block design. In 2015, plots were established on a $20 \mathrm{~m} \times 18 \mathrm{~m}$ tilled plot of land and in 2016 plots were established on $25 \mathrm{~m} \times 25$ $\mathrm{m}$ tilled plots of land. The 2016 site was approximately 210 meters southeast of the 2015 site. 


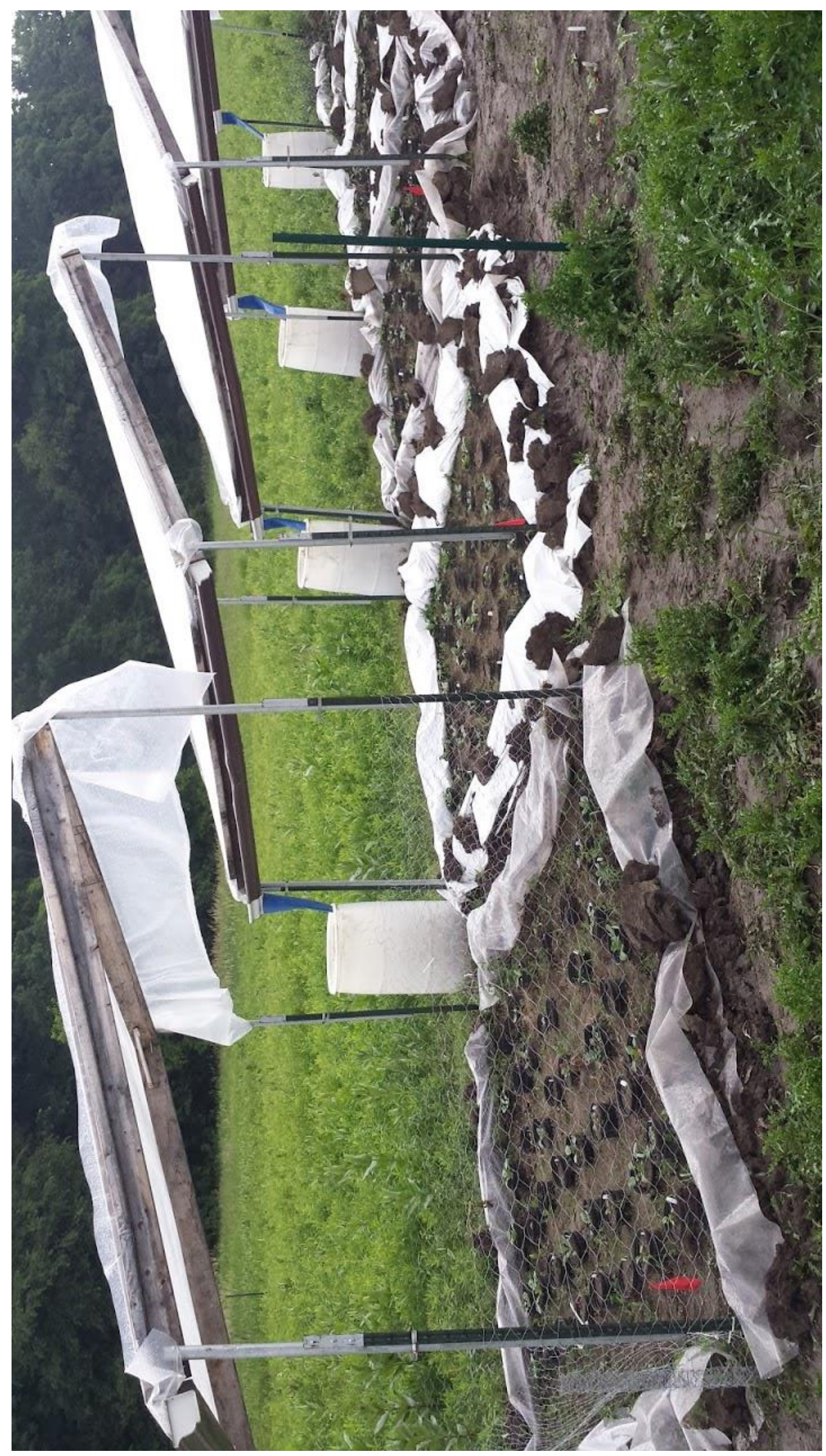

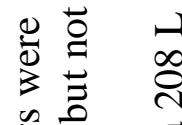

형

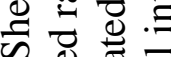

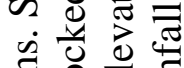

웡응

范 芩

호

政

है

ర

$\circ$ on 3

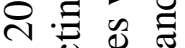

च

n $\Xi \pm$

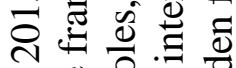

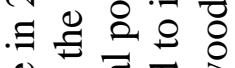

듀 휴

.

밍 की

-

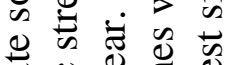

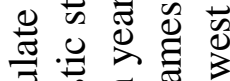

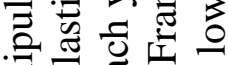

寻艺导的

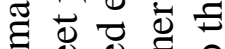

응 응

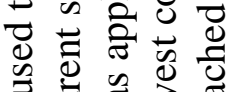

$\Rightarrow$ है है

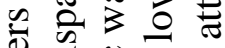

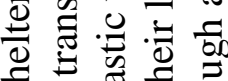

क 든

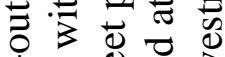

.

渮合西

Ч

든 Ð

흉 매워

이예

¿

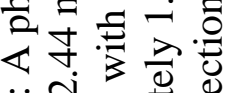

긍

궁

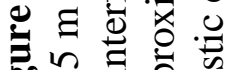
ప్b. 


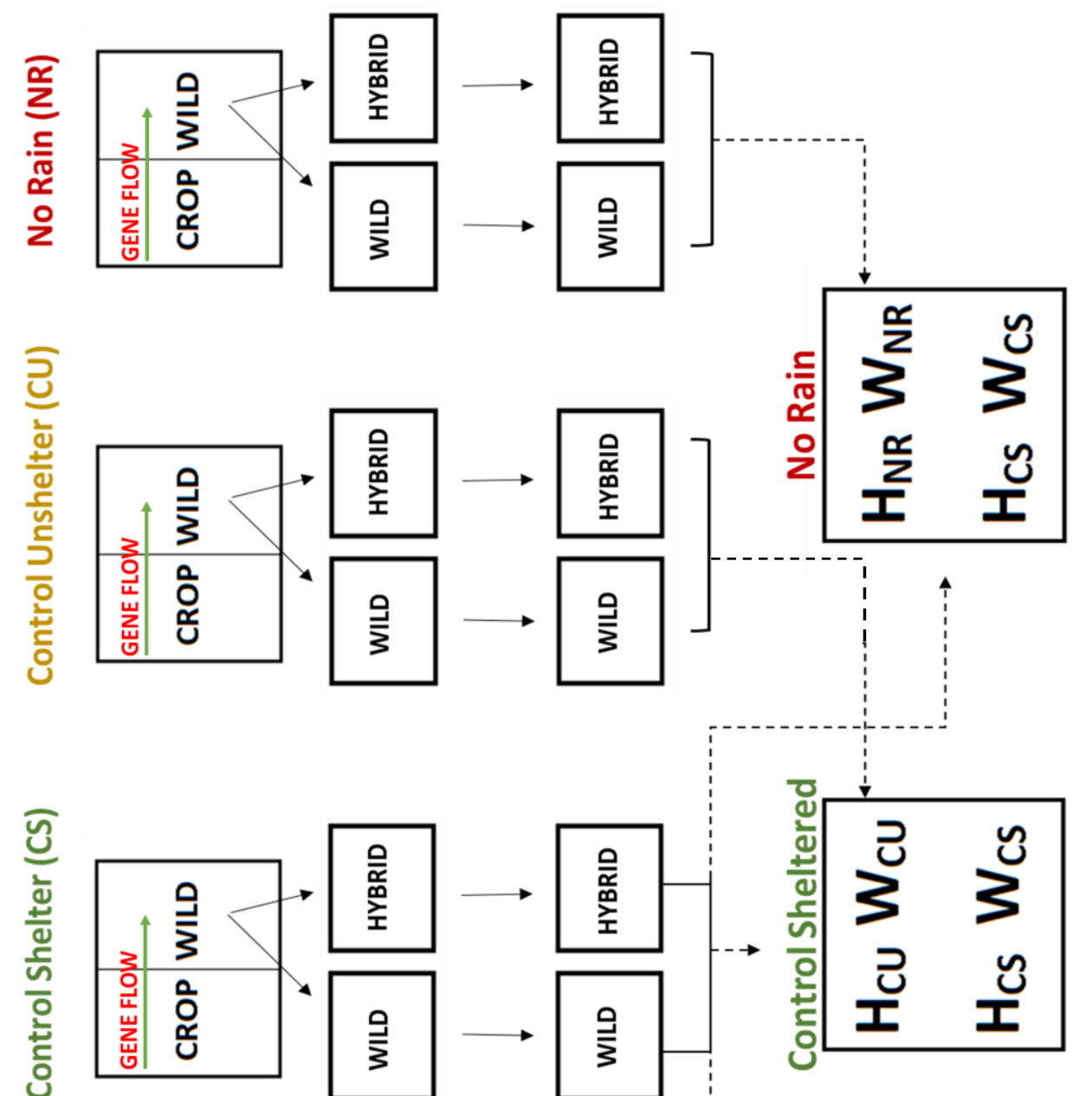

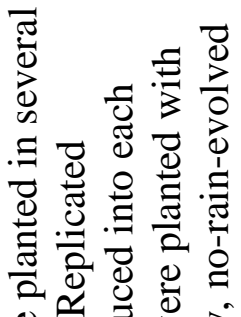

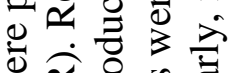

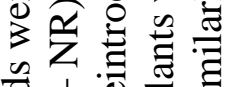

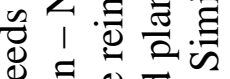
क ज. 당 $3 \frac{0}{0}$

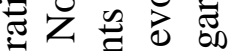

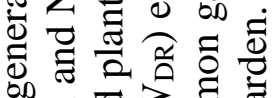

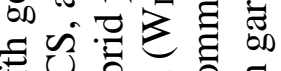

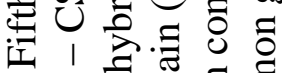

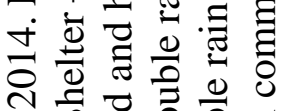

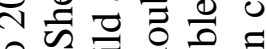
응 을

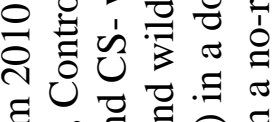
뭉 $\leftrightarrows \overline{0} \approx$

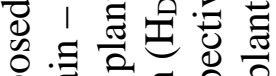
㻖

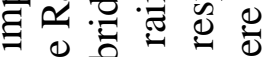

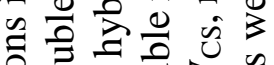

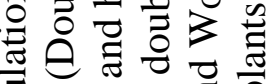
구을

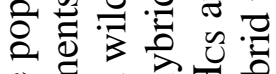

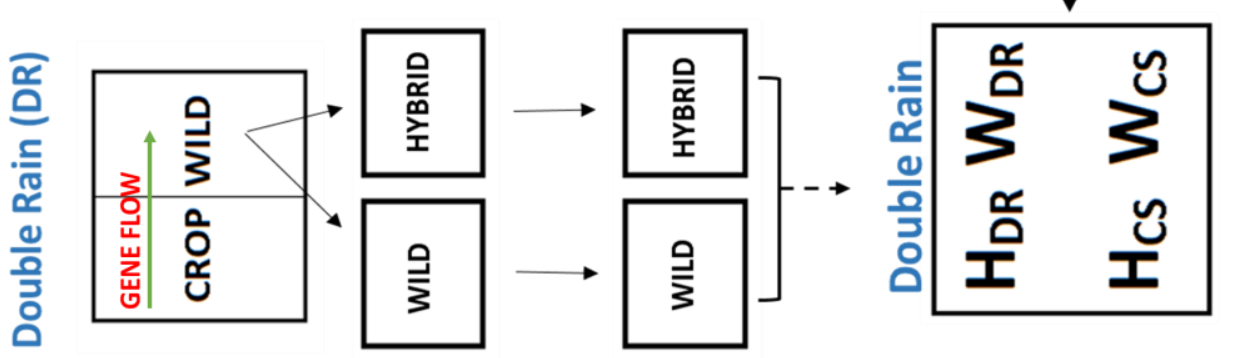

\&

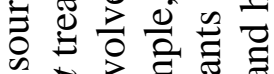

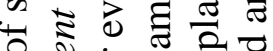

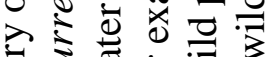
匀

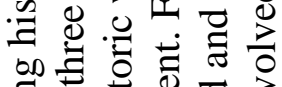
$\Xi$ 可

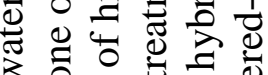

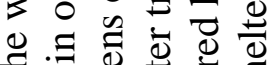

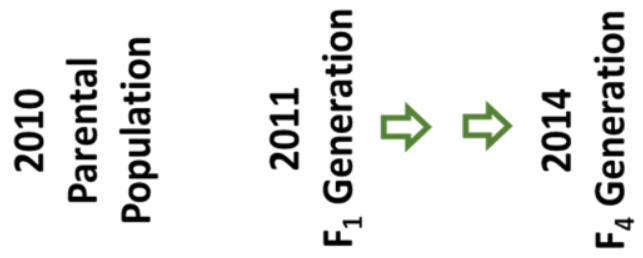

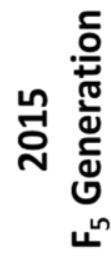

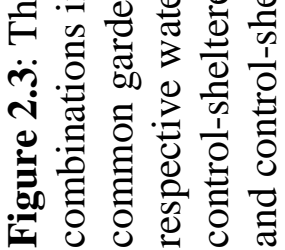



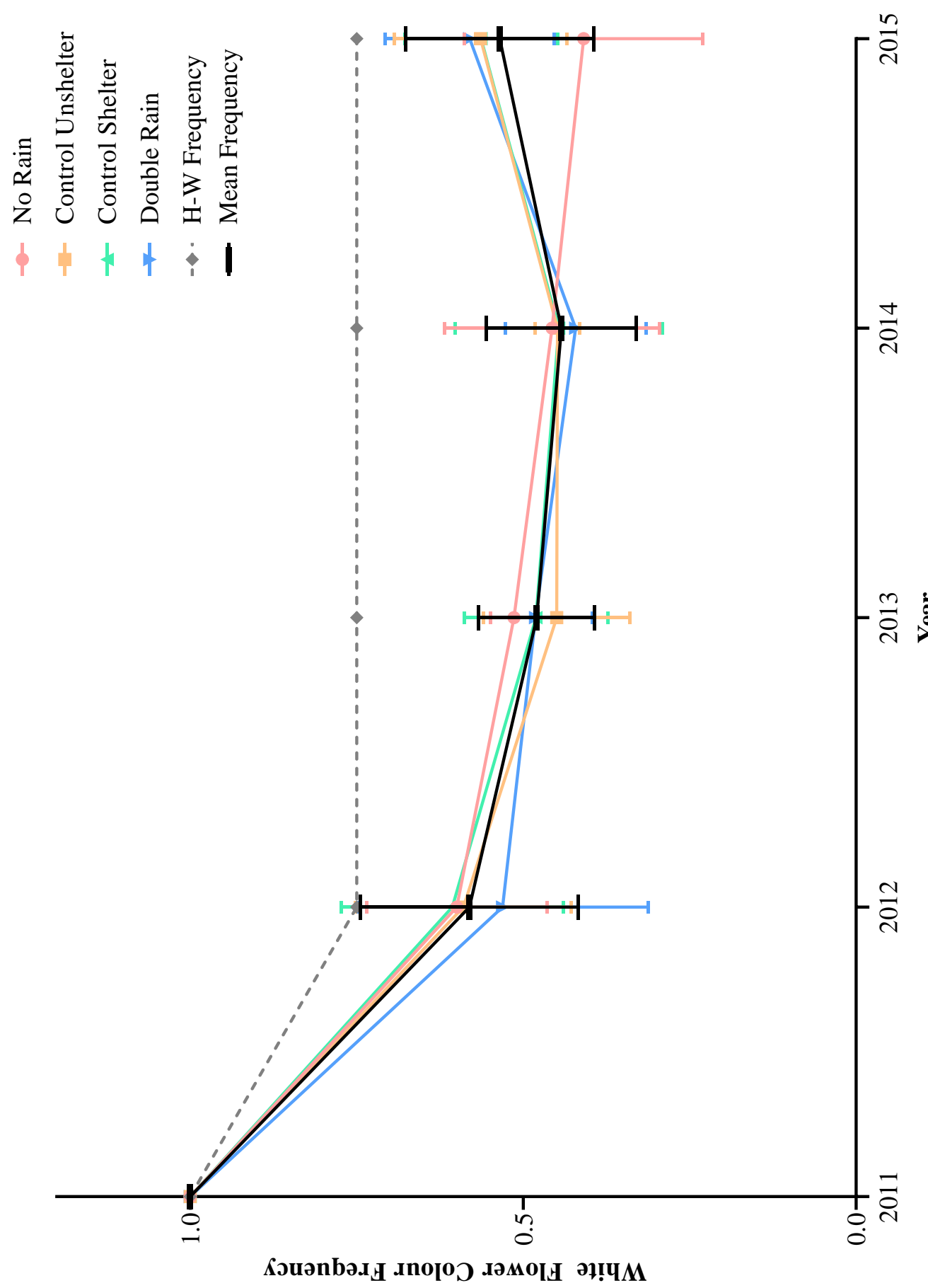

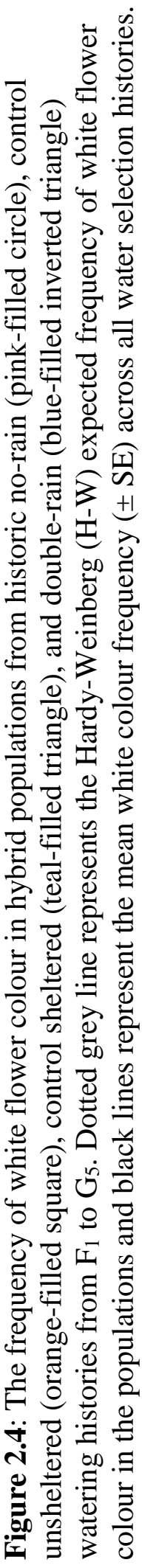




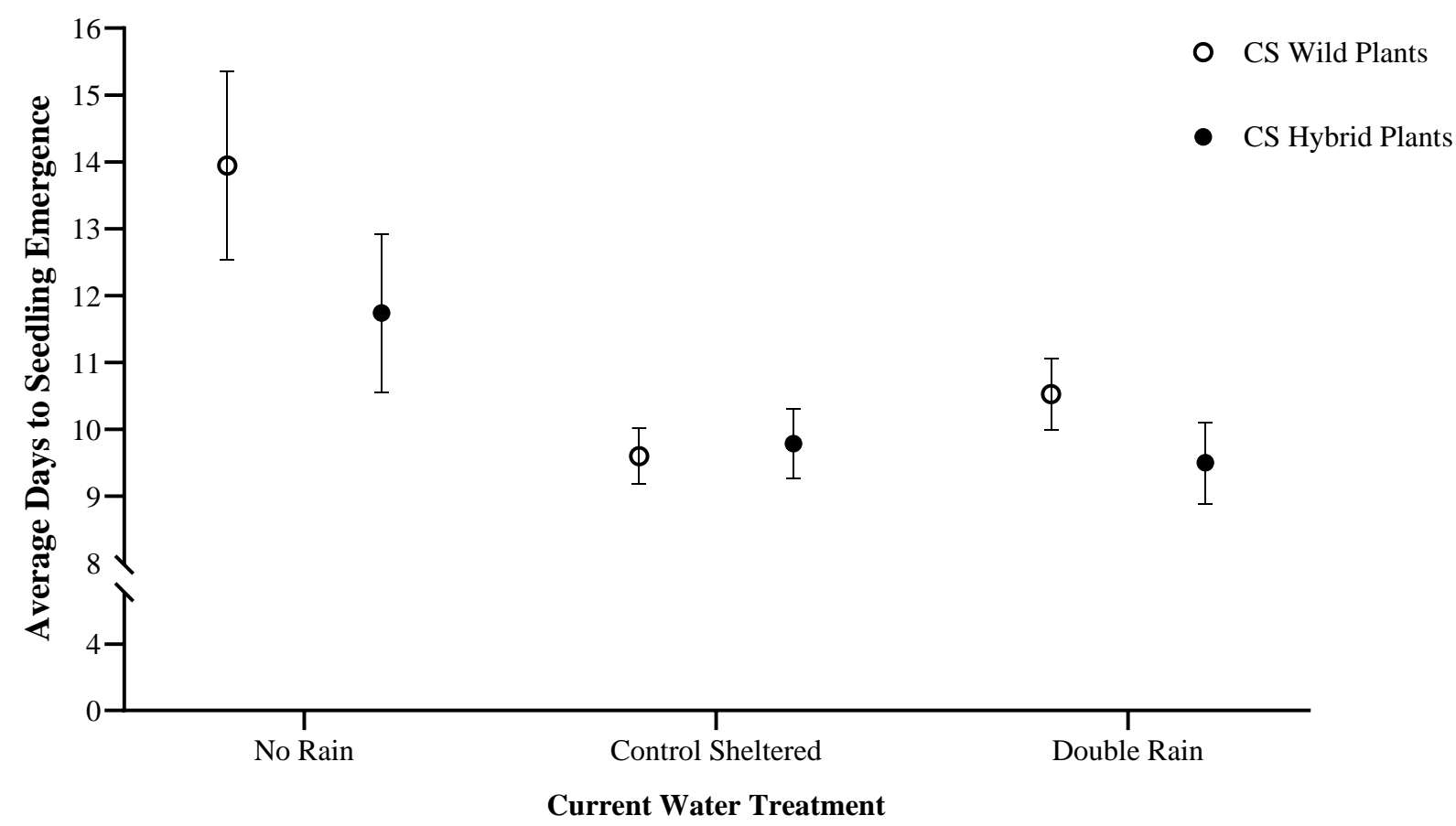

Figure 2.5: Comparing the average number of days wild or hybrid seedlings take to emerge from the soil when invading into novel and control current watering treatment environments. The graph displays the mean days to seedling emergence $( \pm$ SE) of control shelter evolved wild and hybrid plants (wild - open circles, hybrid - filled circles) invading into novel environments (no rain and double rain environments) versus control sheltered environments. 


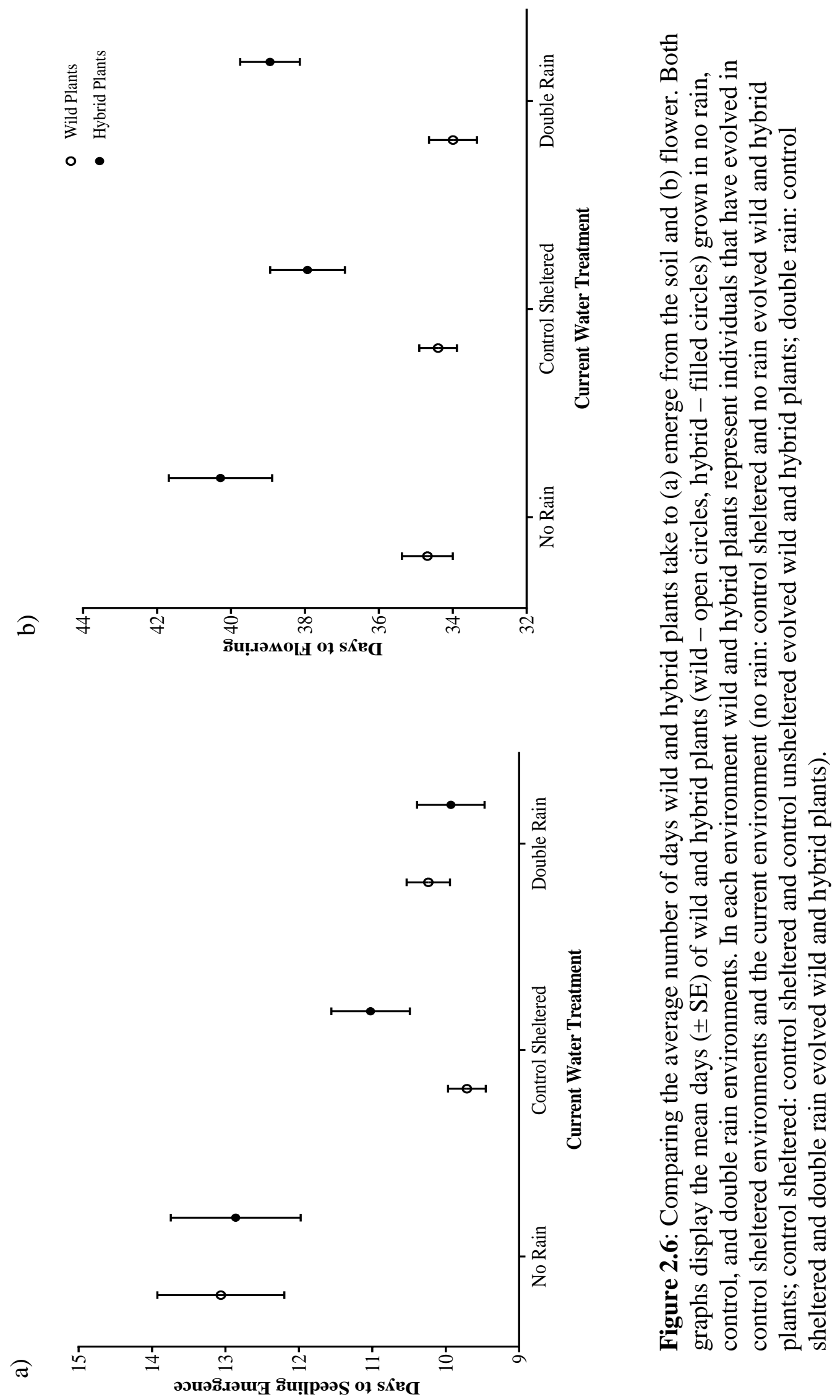



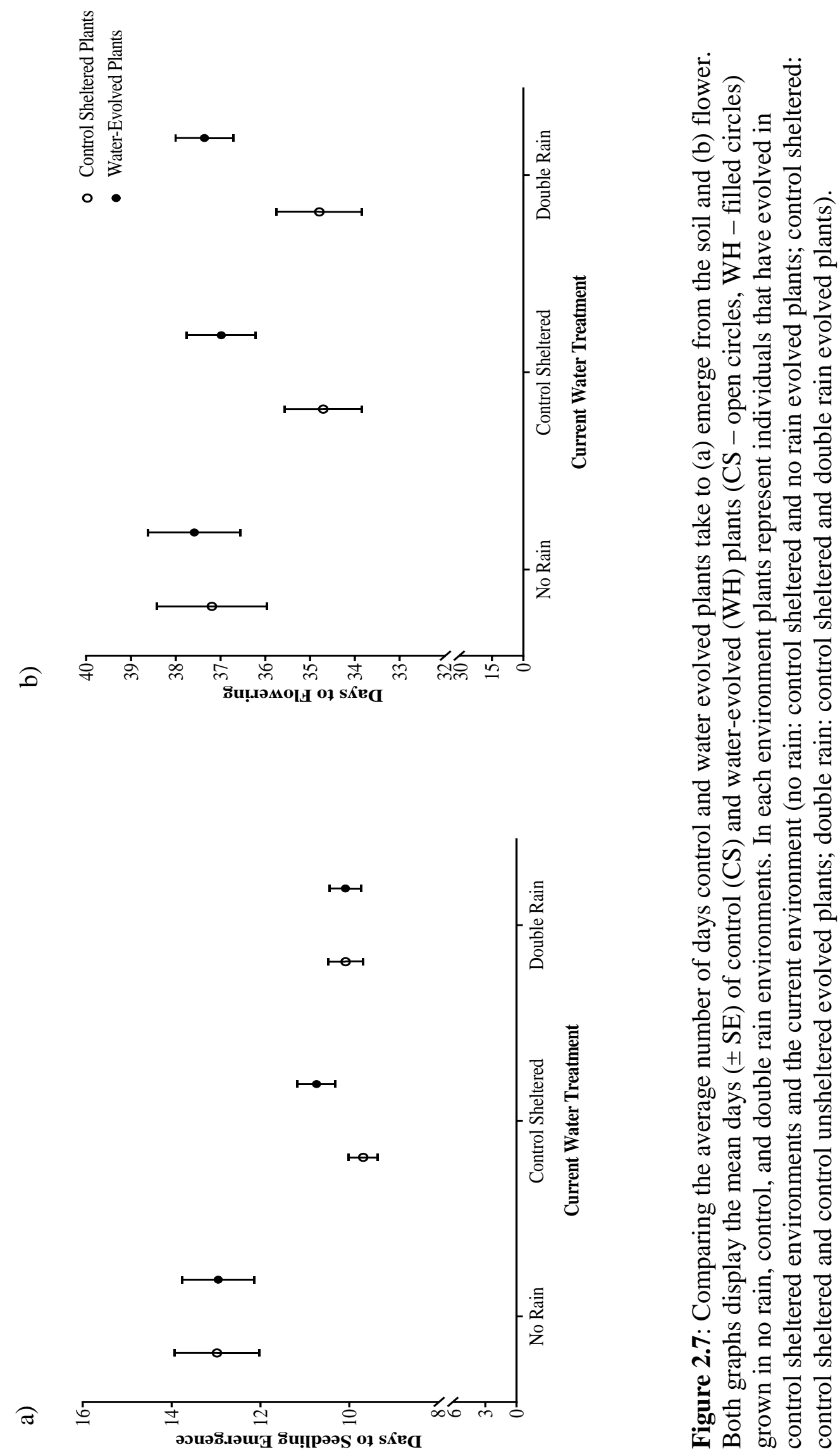

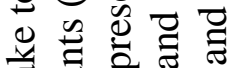

폰 은 귱

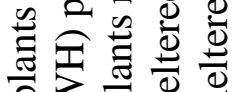

运之运过

రृ

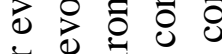

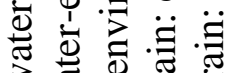

उ 䒕可

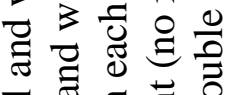

을 ○己 芯芯

我

㐫苛

世渮 ১) हิ

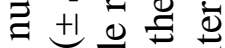
0 $1 \frac{0}{0}$ ส む) ঊ 舀 b 을 읍 灵 㐫 हैं है 己ृ U $\therefore$ 등 웡

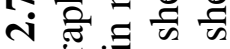

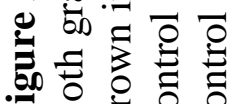
四 ص పू 


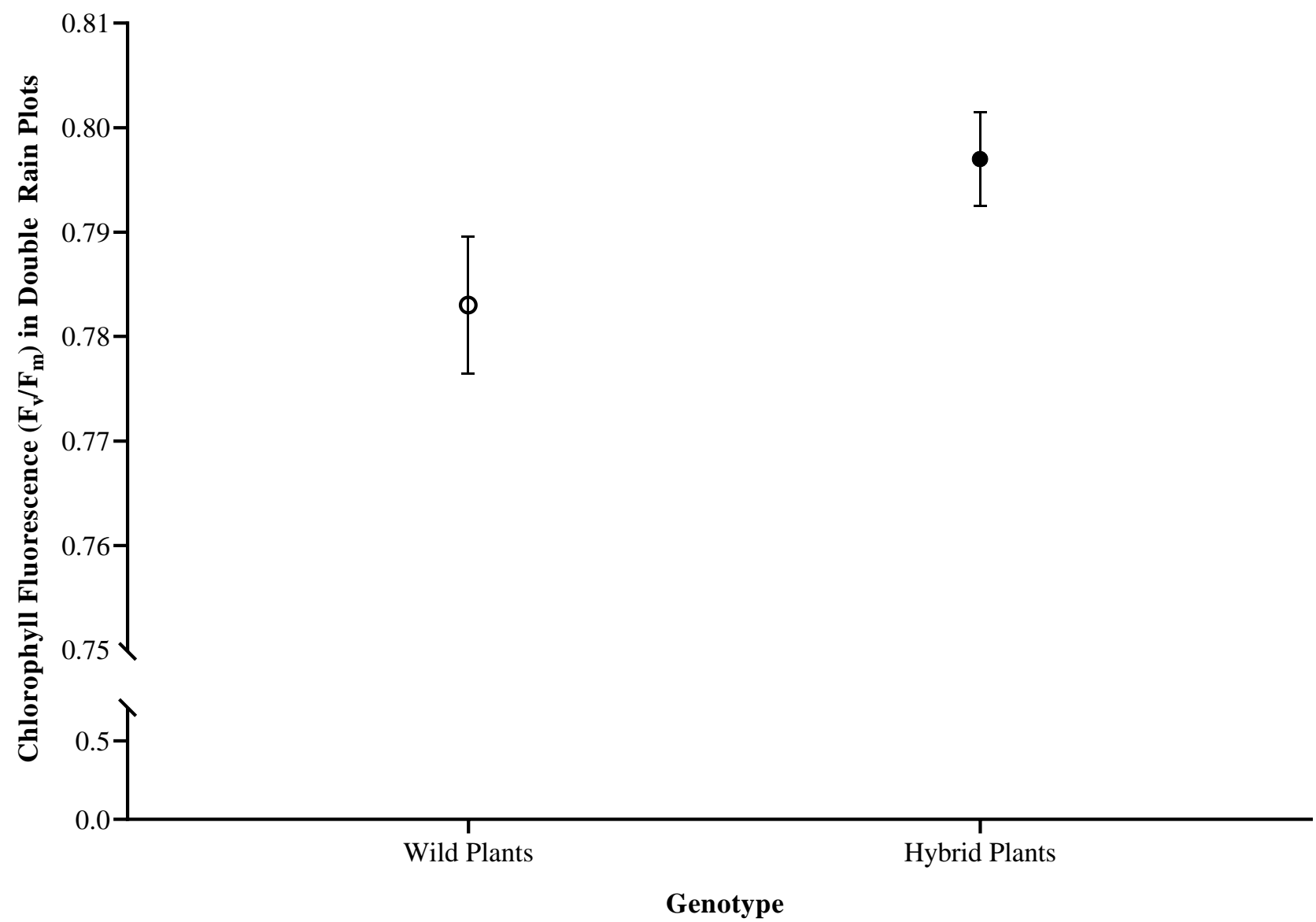

Figure 2.8: Comparing the quantum efficiency of the photosystem II (PS II) of plants that grew in double rain moisture environments. The graph displays average chlorophyll fluorescence $( \pm$ SE) between wild and hybrid plant genotypes (wild - open circle, hybrid - closed circle) when grown in double rain environments. Wild and hybrid plants represent individuals that have evolved in double rain and control environments, collectively. 


\section{Chapter 3: Rate of Evolution Metrics are Sensitive to Environmental Context}

\subsection{Abstract}

Rate of evolution (RoE) metrics attempt to measure the speed at which a population evolves by comparing phenotypic variance $\left(V_{P}\right)$ of populations over a period of time. However, $V_{P}$ is sensitive to environmental context which is overlooked in traditional RoE estimation methods. Although others have acknowledged $V_{E}$ could confound RoE estimates, I present the first study to explicitly measure the environmental sensitivity of RoE and demonstrate the repercussions. I estimated RoE for 40 Raphanus populations that varied in their history of hybridization and environmental context (imposed by an experimental moisture cline) in two common gardens (Years: 2015, 2016). I show that RoE estimates are incorrect when control and evolved populations are grown in two locations, a common mistake in the contemporary evolution literature. Moreover, depending on the particular common garden environment, RoE estimates can differ significantly in magnitude and direction - particularly for wild populations. Environmentally-sensitive trait expression can confound estimates of evolutionary rate and lead to erroneous conclusions about the contemporary direction and RoE. I suggest modified approaches to the estimation of RoE that minimize or account for trait plasticity.

Key words: common garden; contemporary evolution; haldane; phenotypic plasticity; Raphanus; Rate of Evolution.

*Submitted to Evolution (ISSN - 1558-5646, Manuscript ID 18-0794) 


\subsection{Introduction}

Adaptive evolution is expected to proceed at a rate that is proportional to both the quantity of genetic variation and the strength of selection imposed upon a population (e.g., Gingerich 2001; Frankham 2005; Kinnison and Hairston 2007; Campbell et al. 2016a). Thus, it is well documented that phenotypes of genetically diverse populations evolve greater divergence from control populations than genetically depauperate populations (Hard et al. 1993; Sakai et al. 2001; Campbell and Snow 2009; Lanfear et al. 2014). Moreover, populations under stronger selection often exhibit greater divergence than populations under weaker selection (Nosil et al. 2009; Sherrard et al. 2009). These patterns of evolutionary divergence have been repeatedly quantified, such that a substantial body of literature now documents the pace of contemporary evolution (e.g., Losos et al. 1997; Reznick et al. 1997; Hendry and Kinnison 1999; Bone and Farres 2001; Carroll et al. 2001). Upon reviewing the contemporary evolution literature, I noticed that although phenotypic expression is frequently documented to be sensitive to environmental context (Via and Lande 1985; Via et al. 1995; Falconer and Mackay 1996; Lande and Shannon 1996; Conner and Hartl 2004), published estimates of evolutionary rates often fail to address phenotypic plasticity in their experimental design or quantitative analysis (although see Dlugosch and Parker 2008). In part, this is because current metrics used to calculate evolutionary rates do not account for the influence of environment on phenotypic variance $\left(V_{P}\right)$. Here, I explore the consequences of ignoring environmental variation when attempting to estimate contemporary rates of evolution.

Calculations of evolutionary rates are based upon the average phenotypic difference between two populations (usually described as $\Delta \bar{x}$ but which can also be expressed as $\Delta V_{P}$ ), relative to the time since isolation (i.e., $\Delta t$ ) and are commonly measured in darwins or, more recently, haldanes (Haldane 1949; Gingerich 1983, 1993). Phenotypic variance $\left(V_{P}\right)$ is comprised of both genetic variance $\left(V_{G}\right)$ and environmental variance $\left(V_{E}\right)$ (Conover and Schultz 1995; Browne et al. 2002; Hartl and Clark 2007), where the magnitude of $V_{G}$ reflects the degree to which there is heritable variation in traits and heritable variation in plastic trait responses to environmental variation (sic Bradshaw 1965; Strand and Weisner 2004; Byers 2008) and $V_{E}$ reflects the degree to which variation in traits is environmentally sensitive (without genetic control) (Hartl \& Clark 2007). Current rate of evolution metrics do not account for heritable or non-heritable phenotypic plasticity but rather treats $\Delta \bar{x}$ as synonymous with environmentally 
insensitive genetic variation expressed in the phenotype. However, phenotypic plasticity (either heritable or not) as an evolved-genetic response is a well-documented, common, and adaptive strategy for many plants (e.g., Sultan 1995; Williams et al. 1995; Richards et al. 2006;

Ghalambor et al. 2007; Moczek et al. 2011).

There are a variety of ways in which environmentally sensitive trait variation may be overlooked in experimental design of rate of evolution studies. Historically, divergence rate estimates were used to measure patterns of fossil evolution and, as such, were always calculated from measurements of organisms grown in separate environments, at separate time points, because of the limited samples available (Marshall and Corruccini 1978; Gould et al. 1989; Clyde and Gingerich 1994). The practice of calculating evolutionary rate estimates comparing the evolutionary divergence of populations grown in environments is employed in estimates of contemporary rates of evolution, as well (e.g., Hendry and Kinnison 1999; Bone and Farres 2001; Haugen and Vøllestad 2001; Santos et al. 2013; Presotto et al. 2016). Yet, comparisons of genetically identical organisms grown in different locations or times would provide inaccurate estimates of evolutionary rates when traits are environmentally sensitive. Similarly, estimates of evolutionary rates from individuals grown in a single common garden environment can only capture the degree of phenotypic differentiation in a single environmental context (e.g., Franks et al. 2007; Dlugosch and Parker 2008; Campbell et al. 2009b) and thus will not represent universal estimate of evolutionary rates possible even from a single set of population comparisons. Instead, evaluating differentiation in multiple common garden environments, with a diversity of genotypes, would potentially capture a wider expression of phenotypic plasticity and thus provide a range of estimates of evolutionary rate. Although studies that measure evolutionary rates have acknowledged the role of environmental context in their estimates (Hendry and Kinnison 1999; Bone and Farres 2001; Hendry et al. 2008; Gorné and Díaz 2017), I did not encounter a study in my literature searches that has attempted to measure the consequence of environmental context on rates of evolution.

\subsubsection{Objectives}

I created 40 experimental populations with relatively low or high genetic diversity (using inter-specific hybridization) that experienced diverse selection gradients (imposed by 
experimental watering treatments; (Campbell et al. 2016b) and allowed the populations to evolve for four generations to determine:

(1) Do evolutionary rate estimates for a variety of traits differ among populations with divergent histories of selection and/or hybridization?

If traits are environmental insensitive then I expected rate of evolution (RoE) estimates between populations would not differ (i.e., result in evolutionary rate estimates of zero). However, if watering history imposed selection on these traits, then I expected evolutionary rate estimates to diverge from control populations. Similarly, if hybrid populations possessed and retained greater levels of genetic diversity (i.e., through extensive gene flow), I expected hybrid populations to evolve more rapidly than wild populations in response to environmental selection pressures (i.e., watering history).

(2) Are evolutionary rate estimates correlated when evolved and control populations were grown in separate environments versus when evolved and control populations were grown in common gardens?

I expected, if environmental context (i.e., the environment references and evolved plants are grown) does not confound rate of evolution estimates then, correctly calculated RoE values (i.e., reference and evolved plants in the same common garden) would be highly correlated to incorrectly calculated RoE values (i.e., references and evolved plants in different common gardens); suggesting $\mathrm{V}_{\mathrm{E}}$ does not affect evolutionary rate estimates.

(3) Do evolutionary rate estimates for a variety of traits differ among environments?

I anticipated, if common garden environment did not influence the trait response (i.e., do not exhibit plasticity), RoE estimates would be similar between common garden years for any given trait. 


\subsection{Methods}

\subsubsection{Study Species}

Cultivated radish (Raphanus sativus L.) and wild radish (or jointed charlock, Raphanus raphanistrum $\mathrm{L}$.) are annual, insect pollinated, self-incompatible, diploid species that can hybridize (Panetsos and Baker 1967). Cultivated radish is an annual crop species that flowers late in the growing season, exhibits low rates of dormancy and rapid germination, and grows large, edible hypocotyls (i.e., roots; Snow and Campbell 2005). Wild radish, in contrast, flowers early in the growing season, has a long-lived seed bank, exhibits seed dormancy, and variable germination times after soil disturbance, and develops a relatively small, inedible hypocotyl. Wild radish is a common weed in agricultural systems in temperate North America, and Europe, also found in disturbed and costal sites in temperate climates (Holm 1997). The success of their hybrid derivative ( $R$. raphanistrum $\mathrm{x}$ R. sativus) as an aggressive weed is apparently environmentally dependent (Campbell et al. 2006; Hegde et al. 2006; Campbell et al. 2009a; Campbell et al. 2009b; Ridley and Ellstrand 2010; Hovick et al. 2012). Moreover, hybrid radish populations tend to evolve faster than wild radish populations but this has varied with selection pressure (Campbell et al. 2009a; Campbell et al. 2009b). Since fitness of crop-wild hybrid radish relative to wild radish has varied with diverse moisture, temperature and ecological contexts (Campbell et al. 2006; Hovick et al. 2012), I chose to manipulate moisture conditions in field plots to explore the influence of moisture on the relative fitness of crop-wild hybrids.

\subsubsection{Seed History of Wild and Hybrid Radish Populations Used in my Experiment}

Ancestral populations (i.e., $\mathrm{F}_{0}$ generation) of wild radish (Raphanus raphanistrum) were collected from greenhouse populations that were grown for several generations near Binghamton, NY, USA (Conner and Via 1993). The crop radish (Raphanus sativus) cultivar used was Red Silk (Harris-Moran Seed Company, Modesto, CA, USA). As in Campbell et al. (2016), in 2010, both cultivated and wild plants (nine seedlings per genotype) were planted in 36 plots as part of a randomized block design at the Waterman Farm at Ohio State University in Columbus, Ohio USA, within a larger experiment (Sneck 2012; Campbell et al. 2016; Fig. 3.1). Seedlings were planted in one of four watering treatments with one plot per treatment, per block, for a total of ten blocks. Plots were approximately 200 meters apart to minimize gene flow among plots; although likely negligible, some gene-flow may have occurred (see chapter 2, page 20). In the $\mathrm{F}_{0}$ 
generation, gene-flow naturally occurred within mixed plots of wild and cultivated-crop plants and gave rise to the first generation (i.e., $\mathrm{F}_{1}$ ) of wild and crop-wild hybrid ( $R$. sativus $\mathrm{x} R$. raphanistrum) seeds (Teitel et al. 2016a). As previously described (e.g., Campbell et al. 2016b; Teitel et al. 2016a), I manipulated soil moisture using one of four watering treatments within these plots for three generations, to impose a natural selection experiment on replicated wild and crop-wild hybrid radish populations:

(1) Control Unsheltered (CU): To establish a control precipitation treatment, ambient rainwater fell on un-manipulated populations.

(2) Control Sheltered (CS): To determine the effect of a rain-out shelter (but not manipulation of moisture availability) on plant growth, ambient rainwater, collected from the shelter, was applied to the plot.

(3) No rain (NR): To create relatively dry soil conditions, water collected from NR shelter barrels was withheld.

(4) Double Rain (DR): To create relatively wet soil conditions, water collected from DR and NR shelters was applied to DR plots; that is, double the ambient rainfall.

The $F_{1}$ and following generations of wild and crop-wild hybrid seeds were grown at the Koffler Scientific Reserve (KSR) on Jokers Hill, King City, Ontario, Canada (lat. $44^{\circ} 0^{\prime}$ N, long. 79³' W; elevation $285 \mathrm{~m}$ asl) when the Campbell lab relocated from Columbus, Ohio to Toronto, Ontario, Canada. At KSR, $\mathrm{F}_{1}$ seeds from $40 \mathrm{~F}_{0}$ plots were grown in germination trays in a hoop-house. Wild and hybrid $\mathrm{F}_{1}$ seedlings were grown to the two-leaf stage, transplanted into 20 wild and 20 hybrid plots scattered across KSR and were exposed to the same rainfall treatments as in Ohio for an additional three generations. Shelters were placed at least $200 \mathrm{~m}$ apart to reduce gene flow among plots, as in the $\mathrm{F}_{0}$ generation. Since wild radish has long-lived seed banks and since annual regeneration of populations was a result of seeds that dropped to the ground and naturally germinated, fruits collected from the pedicels of senescing plants in 2015/2016 could have belonged to one of three generations ( $F_{2}$ to $\left.F_{5}\right)$. For simplicity, I refer to these plants as $\mathrm{G}_{2}-\mathrm{G}_{5}$ generation seeds. Wild and hybrid $\mathrm{G}_{5}$ seeds were collected from $\mathrm{G}_{4}$ plants in fall 2014 and used in both 2015 and 2016 experiments. 


\subsubsection{Common Garden Set-up}

To estimate the rate at which phenotypes diverged after three generations of selection and determine the confounding effect non-heritable plasticity (i.e., $V_{E}$ ) had on evolutionary rate metrics, I grew two common gardens (one in the 2015 growing season and another in 2016) of $\mathrm{G}_{5}$ wild and hybrid plants. In 2015 , ten common garden plots $(3.5 \mathrm{~m} \times 3.0 \mathrm{~m})$, treated as blocks, were tilled and planted with a total of 120 seeds per block; from each $\mathrm{G}_{5}$ genotype by watering history combination, 15 seeds were randomly selected from five populations (Fig. 3.1). On June $1-2,2015$, I planted seeds in the soil in a $10 \times 12$ grid, with $30 \mathrm{~cm}$ spacing between plants, arranged in a randomized, complete block design. Common garden rainfall was not manipulated in either common garden and plots were weeded to reduce competition. In 2016, I replicated the 2015 experiment at a second site at KSR. The second common garden was tilled and ten experimental blocks $(3.5 \mathrm{~m} \times 3.0 \mathrm{~m})$ arranged in a randomized, complete block design on May 20th and May 24th, 2016. In 2016, every genotype by environment combination from 2015 along with three crop seeds (Raphanus sativus) were planted in each block. However, these plants were removed from the analysis due to lack of replication across years. Due to limited seed stock, I planted 100 seeds per block in each common garden. I harvested the plants as they senesced, when flowering was complete and at least ten fruits were ripe, if the plant produced at least 10 fruits or when all fruits were ripe, if the plant produced less than 10 fruits. At the end of the growing season (October 15 $5^{\text {th }}, 2015$ and 2016), all remaining plants were harvested. Natural rainfall varied over the growing season between common garden years, with a cumulative rainfall of $307.8 \mathrm{~mm}$ in 2015 and $206.7 \mathrm{~mm}$ in 2016 (nearest weather station: Buttonville, Ontario 4351'39.000" N , 79²2'07.000" W; Government of Canada 2018).

\subsubsection{Trait Measurement}

Flower colour, a simply inherited trait, differs between wild and crop radish plants and is a visual marker to track crop trait introgression in hybrid populations (Snow et al. 2001; Campbell et al. 2006). Wild radish (Raphanus raphanistrum) is homozygous recessive for yellow flower petal colour and crop radish (R. sativus) is homozygous dominant for white or pink flower petal colour (Panetsos and Baker 1967; Kay 1976; Stanton et al. 1989). In hybrid populations, the white petal colour exhibits Mendelian dominance over the yellow petal colour, and therefore allows us to track crop allele persistence (Panetsos and Baker 1967; Stanton et al. 
1989) into advanced populations of crop-wild Raphanus hybrids. Hues of pink petal colour is controlled by two additional loci (Panetsos and Baker 1967) but variation in pink hues was not tracked in this experiment.

Radish hybrids can be heterozygous for a reciprocal translocation that can affect chromosome pairing during meiosis (Panetsos and Baker 1967; Campbell et al. 2006). This translocation can affect fertility and produce up to $~ 60 \%$ aborted pollen grains in hybrid progeny (Snow et al. 2001; Campbell et al. 2006). After four generations, I compared hybrid pollen fertility to that of wild plants to determine the rate of evolution in hybrid pollen fertility across environments. To assess pollen viability of $\mathrm{G}_{5}$ hybrid populations relative to the pollen viability of wild radish, I collected a single, newly opened flower from each plant ( $\mathrm{n} 1000$ plants/year) during August 2015 and July-August 2016 between the hours of 8:00 am and 12:00 pm and refrigerating at $2^{\circ} \mathrm{C}$ until processing. Upon staining, two anthers were collected and wiped on microscope slides (VWR VistaVision, Radnor, PA, USA). Slides were stained (Alexander 1969) and stored in slide boxes. After staining, I measured pollen fertility by categorizing at least 100 pollen grains per plant as either the number of aborted or fertile pollen grains using a compound microscope (H550L, Nikon @, Japan).

I monitored each seed daily to record the date of seedling emergence from the soil and first flower during the experimental period (June to October 15, 2015 and June to August 26, 2016). From this, the days to seedlings emergence and age at first flowering (i.e., number of days between anthesis and emergence) was calculated. Additional life-history traits (e.g., longest leaf length, stem diameter) were measured at the date of first flower and damage caused by herbivory. Given that the results associated with leaf length, stem diameter, and herbivory damage were consistent with the phenology of seedling emergence and flowering, results of leaf length and stem diameter are included in Appendix 3.

To measure the photosynthetic performance of plants in the different watering treatments, and evaluate whether water stress or excess water influenced photosynthesis, I measured the dark-adapted quantum efficiency of photosystem II (PSII) as the ratio of variable $\left(\mathrm{F}_{\mathrm{v}}\right)$ to maximal $\left(\mathrm{F}_{\mathrm{m}}\right)$ chlorophyll fluorescence (Maxwell and Johnson 2000). Because all reaction centres were fully oxidized by shading prior to measurement, $\mathrm{F}_{\mathrm{v}} / \mathrm{F}_{\mathrm{m}}$ represents the maximum capacity of PSII to absorb light energy. In both years, I took outdoor measurements using a portable fluorescence 
meter (Handy PEA fluorometer, Hansatech Instruments Ltd., King's Lynn, UK). Prior to experimental measurement, I randomly sampled 100 plants to determine a standard curve of the minimum time it took reaction centers to become fully oxidized; a dark adaptation period (period without light) where the photosynthetic pathway is free of electrons (i.e., the energy needed to drive photosynthesis) leaving reactions centers are fully open ready to accept more electrons) (Goltsev et al. 2016). When reaction centers are fully oxidized, fluorescence remains consistent; this occurred after approximately 10 minutes for radish plants. Because all reaction centres were fully oxidized by shading prior to measurement, $\mathrm{F}_{\mathrm{v}} / \mathrm{F}_{\mathrm{m}}$ represents the maximum capacity of PSII to absorb light energy. In both years, I took outdoor measurements using a portable fluorescence meter (Handy PEA fluorometer, Hansatech Instruments Ltd., King's Lynn, Norfolk, UK). Then, for the experiment, I non-destructively sampled quantum efficiency of PSII on a subset of twenty-four plants (three plants per historical environment $\mathrm{x}$ genotype combination) per block for a total of 240 plants across the whole experiment in 2015 and another 240 plants in 2016. In random order, I measured plants after a 10-minute dark adaptation period. Measurements were collected on July 7, 2015 and between June 29 - July 3, 2016, between 8:00am and noon.

\subsubsection{Evolutionary Divergence Rate Metrics}

Because I compared independently evolving populations, I calculated synchronic rates of evolutionary divergence (Hendry and Kinnison 1999; Bone and Farres 2001), using the following equation:

$$
\text { haldanes }(h)=\frac{\left[\left(\frac{\ln \bar{x}_{2}}{s_{p}}\right)-\left(\frac{\ln \bar{x}_{1}}{s_{p}}\right)\right]}{t_{2}-t_{1}} \quad \text { (Equation 1), }
$$

where the mean trait values of CS wild and CS hybrid populations were represented by $\bar{x}_{1}$ and trait values of DR-, NR-, or CU-evolved wild and hybrid populations were represented by $\bar{x}_{2}$ in Equation 1. By calculating haldanes, mean trait evolution was standardized by incorporating pooled trait variances $\left(s_{p}\right)$ and measuring evolutionary change through time $\left(\mathrm{t}_{2}-\mathrm{t}_{1}=5\right.$ generations, or $\mathrm{F}_{0}-\mathrm{F}_{5}$ ) (Haldane 1949; Gingerich 1993, 2001). I calculated the natural log of trait values to reduce heteroscedasticity in the dataset since standard deviations are typically expected to increase with the mean, particularly for morphological traits (Wright 1968; Hendry and Kinnison 1999). Finally, evolutionary rates, calculated in darwins $(d)$, are presented in Appendix 2. 


\subsubsection{Statistical Analysis}

To determine if phenotypic traits differed in response to an environment (year) by watering history (WH: NR, CU, CS, DR) by genotype (wild versus hybrid) effect, and block, I ran a full-factorial, linear, mixed-model MANOVA (using the manova function in the stats, $\mathrm{R}$ Core Team) on z-score transformed trait values for each trait (i.e., emergence time, flowering time) and applied further transformations (Tables 3.1, 3.2, and 3.3), as necessary, to meet assumptions of normality (i.e., boxcox transformations in the car package in R-Studio ver. 1.0.1430, Fox 2011). To determine significant pair-wise differences, I first ran a full-factorial, mixed-model ANOVA for each phenotypic trait (using the lmer function in R-Studio, R Core Team ) followed by a post-hoc Tukey multiple comparisons of means tests (function TukeyHSD in the emmeans package, R Core Team). In the model, I ran WH, genotype, and year (because it was the primary effect I was interested in) as fixed factors and block as a random factor. Due to the non-orthogonal nature of the data (i.e., not balanced), type III ANOVA results applied a Kenward-Roger's adjustment for computing the degrees of freedom (Luke 2017).

To test whether reaction norms varied among radish populations for each mean trait value, I ran a linear, mixed-model, repeated-measures ANOVA. Considering the non-orthogonal nature of this particular dataset, I first removed the effect of block by calculating the residuals of the model for each trait with block as the only independent factor. Next, I combined WH and genotype into a single factor and ran the model with each residual trait value (i.e., with block effect removed) against year and WH genotype as fixed factors, and population as the repeated measure ( $l m$ and aov functions in the stats package, R Core Team; statistical output and results are presented in Appendix 4).

To determine the degree to which the environment of the reference population (i.e., the garden in which control sheltered populations were grown) influences the estimate of evolutionary divergence of the selection populations, I explored the correlation among divergence rates calculated incorrectly and correctly - and I describe these calculations next. As mentioned in the introduction, sometimes published studies estimate evolutionary rates from populations that are grown in different environments, an incorrect approach to estimate contemporary evolutionary rates. I simulated this type of study by calculating "incorrect" divergence rates by using reference (CS wild or hybrid) populations grown in 2015 to estimate 
the evolutionary divergence of selection (NR, CU, DR) populations grown in 2016 and vice versa, by using reference (CS wild or hybrid) populations grown in 2016 to estimate the evolutionary divergence of selection (NR, CU, DR) populations grown in 2015. I then compared these incorrect divergence rates to correctly estimated divergence rates (where reference and selected populations are grown in a common garden) by running simple linear regressions (using SPSS) on correct and incorrect divergence rates where the selection populations were grown in the same common garden (e.g., 2015 or 2016) but the reference populations were either correct or incorrect for each trait (emergence time, flowering time, stem diameter and leaf length).

To determine if rates of evolutionary divergence differed in response to an environment (year) by watering history (WH) by genotype (wild vs hybrid effect), I ran a mixed-model, repeated-measures MANOVA, with WH, genotype, and their interaction as the between-subjects effects and year and its interactions with WH and genotype as the within-subject effects (IBM SPSS Statistics 24, Chicago, USA). After determining a significant year interaction for all traits (i.e., emergence time, flowering time), I ran separate full-factorial, repeated mixed-model ANOVA for each phenotypic trait (GLM function IBM SPSS Statistics 24, Chicago, USA). To determine significant pair-wise differences, I ran a post-hoc Tukey multiple comparisons of means test. Similarly, I ran a full-factorial, repeated-measures ANOVA for the frequency of white petalled plants with WH as the main between-subjects effect and year and its interaction with WH as the within-subjects effect. Due to smaller datasets of the traits chlorophyll fluorescence and pollen fertility, testing for differences in chlorophyll fluorescence and pollen fertility divergences rates between years only was done using a Mann-Whitney U-Test for unpaired data due to the non-parametric distribution of the data (function wilcox.test in the stats package, R Core Team; SI - Appendix 2). I did not compare chlorophyll fluorescence and pollen fertility among genotypes or historical watering treatments because of small sample size within each experimental level within a treatment.

Finally, to determine if 2015 plant trait divergence rates predicted 2016 plant trait divergence rates, I ran simple linear regressions (using cor.test and plot functions in the stats package, R Core Team) on divergence rates between years for each trait (emergence time, flowering time, white flower colour frequency, pollen fertility, and chlorophyll fluorescence). Then I tested whether the slope of the correlation of divergence rates between years differed in 
response to genotype, watering history, and their interaction and are presented in Appendix 5 (RStudio ver. 1.0.143). 


\subsection{Results}

\subsubsection{Trait Variation Between Years}

Seedling emergence had significant year $\mathrm{x}$ WH effects where NR plants took longer to emerge in 2015 than 2016 (Table 3.1). Differences in seedling emergence between genotypes did not vary significantly between years, such that emergence of hybrid seedlings was consistently earlier than that of wild plants. In 2015, plants initiated flowering $~ 2.5$ days later, compared to 2016 (with hybrids taking approximately four days longer in 2015 than in 2016; Table 3.1, Fig. 3.2). Finally, there were significant year $\mathrm{x}$ genotype $\mathrm{x}$ WH interactions. Specifically, CS- and CU-hybrid plants flowered eight and five days earlier, respectively, in 2016 than in 2015. Similarly, CS-wild plants flowered four days later in 2016 than in 2015 (Fig. 3.2). Additionally, I found evidence of heritable phenotypic variation and phenotypic plasticity between years for stem diameter and leaf diameter (Appendix 3). Among $\mathrm{G}_{5}$ hybrids, the frequency of plants with white flower petals did not differ significantly between common gardens ( $59 \%$ each year) (Table 3.2). There was a marginally significant difference in frequency of white-petalled plants between years among watering histories (WH); however, there were no significant pair-wise contrasts (Table 3.2). Pollen fertility differed significantly between years where pollen samples included 11\% more fertile pollen grains in 2016 than in 2015. On average, hybrid plants exhibited 9.9\% lower pollen fertility than wild plants across both years (Table 3.2, Fig. 3.3). Further, pollen fertility revealed a significant year by genotype interaction where the difference in fertility between wild and hybrid plants was greater in 2016 ( 11\% difference) than 2015 ( 4\% difference) (Table 3.2, Fig. 3.3).

Complex quantitative traits such as photosynthetic function, time to emergence, and time to flowering showed varying degrees of environmental sensitivity, with significant differences among year by genetic background (either genotype or WH). Hybrid plants exhibited significantly higher quantum efficiency of PSII than wild plants across both years. Further, there was a significant year effect where all radish plants had significantly lower quantum efficiency in 2016 than in 2015 (Table 3.2, Fig. 3.4). However, there were no significant differences between $\mathrm{WH}, \mathrm{WH} \mathrm{x}$ genotype, genotype $\mathrm{x}$ year, or WH x genotype x year on quantum efficiency. 


\subsubsection{Divergence Rates of $G_{5}$ Populations}

Hybridization increased the rate of evolution of days to seedling emergence and days to flower (Table 3.3). Hybrids grown under any watering treatment evolved earlier days to emergence relative to the CS-hybrid phenotype and did so faster than wild plants grown under any watering treatment, relative to CS-wild phenotypes (Table 3.3). Conversely, wild plants grown under any watering treatment evolved later days to flowering relative to CS-wild plants and did so faster than hybrid plants grown under any watering treatment relative to CS-hybrid plants. Watering history did not significantly affect divergence rates of seedling emergence or days to flowering (Table 3.3). Finally, genotype x WH interactions on rates of divergence were not significantly different in date of emergence and first flower (Table 3.3).

The frequency of white-flowered plants did not significantly diverge between populations of NR-, CU-, and DR-evolved and CS hybrids (Table 3.3). The rates of divergence of the quantum efficiency of PSII in NR-, CU-, and DR-evolved wild and hybrid plants qualitatively differed, though, I could not test for statistical differences due to low replication ( $\mathrm{n}=2$, see methods); NR- and CU-wild plants evolved lower quantum efficiency in PSII than CS-wild plants, with the opposite and stronger (i.e., faster) trend in DR-wild plants. In contrast, NR-and CU-evolved hybrid plants evolved higher quantum efficiency in PSII faster than CS-hybrid plants, with the opposite and stronger (i.e., faster) trend in DR-hybrid plants; double rain-evolved wild and hybrid plants evolved

\subsubsection{Correlations Between Divergence Rates When Reference and Selection Populations are Grown in Different Environments versus Common Gardens}

Correlations between divergence rates that were calculated using either reference and selection populations from a common garden versus reference or selection populations from different environments varied from -0.35 to 0.89 (Table 3.4). For most traits, including divergence rate estimates for emergence date, flowering date, and leaf length of the 2015 evolved populations, there was a positive and statistically significant correlation; all of these estimates exhibited relatively weak correlations $(\mathrm{r}<0.7)$. Interestingly, there were stronger positive correlations between correctly and incorrectly estimated divergence rates for evolved populations growing in the 2016 common garden. Finally, in both 2015 and 2016 evolved populations,

correctly and incorrected estimated divergence rates for stem diameter were weakly, significantly 
negatively correlated - that is, incorrectly estimated divergence rates consistently predicted the wrong outcome regarding stem diameter evolutionary rates in both years.

\subsubsection{Consistency Between Environments of Divergence Rates of Populations Grown in Common Gardens}

My data suggest that divergence rates commonly differ (and even contradict each other) when estimated using half-sib families grown in independent common gardens. Rates of evolutionary divergence were contradictory when considering seedling emergence in both years, largely driven by changes in evolutionary rate estimates of wild populations (Table 3.3). In 2015, wild, water-evolved (NR, CU, DR) populations evolved delayed seedling emergence times faster than wild, CS populations. In contrast, in 2016, wild, CS populations evolved delayed seedling emergence times faster than wild, water-evolved populations (Table 3.3, Fig. 3.5). In both years, flowering time divergence rates were significantly different where they evolved in the same direction but at varying magnitudes (Table 3.3). Specifically, water-evolved populations evolved later flowering times faster in 2015 than in 2016 compared to CS-evolved populations. The rate of divergence for a simply inherited trait, the frequency of white flower petals, had opposing estimates between years. In 2015, hybrid, water-evolved populations apparently evolved lower frequencies of white-flowered plants faster than hybrid CS populations but in 2016, the opposite direction of evolution apparently occurred (Fig. 3.6).There were only two traits for which rate of evolution estimates appeared unaffected by the ecological context under which it was measured divergence rates of pollen fertility and chlorophyll fluorescence did not significantly differ between common garden years (pollen fertility: Mann-Whitney $U=9, \mathrm{n}_{1}=\mathrm{n}_{2}=6, \mathrm{P}>0.1$, two-tailed; chlorophyll fluorescence: Mann-Whitney $U=18, \mathrm{n}_{1}=\mathrm{n}_{2}=6, \mathrm{P}>0.1$ two-tailed).

Common garden estimates of divergence in $\mathrm{G}_{5}$ populations also differed depending on their selection history for emergence time but not time to first flower (Table 3.3, Fig. 3.6). Divergence rates of seedling emergence phenology in the 2015 common garden apparently evolved significantly faster (to be later) in NR and DR populations, relative to CS populations. In contrast, divergence rates of emergence time in the 2016 common garden apparently evolved significantly faster (to be earlier) in NR and DR plants, relative to CS plants (Fig. 3.6), with 2016 NR rate estimates being particularly faster than 2015 NR rate estimates. 
Lastly, to determine if genotype $\mathrm{x}$ WH interactions affected estimates of evolutionary rates in $\mathrm{G}_{5}$ plants between common garden years, I compared the magnitude and direction of phenotypic divergence in wild and hybrid plants grown under three watering treatments relative to CS-wild and CS-hybrid plants (Table 3.3). I found the interaction did not significantly differ for the divergence rates of emergence and flowering time.

\subsubsection{Correlations among Divergence Rate Estimates Between Years}

Because divergence rates sometimes differed dramatically among years, divergence rates in 2015 were relatively weakly correlated with divergence rates in 2016 for the frequency of white-flowered plants $\left(\mathrm{r}=0.40, \mathrm{t}_{1,68}=4.67, \mathrm{p}<0.001\right)$, days to emergence $\left(\mathrm{r}=0.23, \mathrm{t}_{1,120}=-5.04\right.$, $\mathrm{p}<0.01)$, and days to flowering $\left(\mathrm{r}=0.53, \mathrm{t}_{1,120}=0.69, \mathrm{p}<0.001\right)$ but were not significantly correlated for pollen fertility $\left(\mathrm{t}_{1,5}=0.69, \mathrm{p}=0.52\right)$, or chlorophyll fluorescence $(1,5=-0.19, \mathrm{p}=0.86)$. 


\subsection{Discussion}

Depending on the genetic variation within a population and its expression patterns across environments, the environmental context under which plants are measured can profoundly alter conclusions that evolution has occurred (e.g., Clausen et al. 1948) and, thus, estimates of the rate at which populations have evolved. My results document that when publications rely on phenotypes from two environments, conclusions about rates of evolutionary divergence can be severely flawed. Despite using relatively similar genetic material in two common gardens, estimates of the magnitude and direction of contemporary evolution differed significantly, particularly for wild populations, due to annual variation in environmental context. In fact, relative to hybrid populations, wild populations had more irregular, sometimes even contradictory, estimates of divergence rates in phenological traits between common gardens (Figs. 3.5-3.7). Therefore, I would reject my null hypotheses for objectives one and three since evolutionary rate estimates and traits were environmentally-sensitive. Furthermore, I found that the strength of correlation between evolved and control populations when grown in separate environments versus when evolved and control populations were grown in common gardens, were not perfectly correlated (i.e., objective three); therefore, I would reject my null hypothesis since environmental context does affect rate of evolution estimates. Differences in phenotypic plasticity among wild versus hybrid genotypes for some, but not all, traits may explain some of the inconsistency in divergence rate estimates. My work is the first to demonstrate that rate of evolution metrics must be judiciously applied, especially when traits exhibit reaction norms or significant amounts of phenotypic plasticity, and thus may more accurately be estimated for traits that perform consistently in populations across all environments (Sultan 2000; Davidson et al. 2011; Liu et al. 2015b). Below I discuss factors that may drive variation in estimates of evolutionary rates (especially heritable and non-heritable expressions of plasticity), careful choice of traits for rate of evolution studies, and propose a modified approach for measuring rates of evolution in heritable, but plastic traits.

\subsubsection{The Puzzle of Inconsistent Estimates of Evolutionary Rates}

Data from two common garden environments revealed three alternative impacts on the consistency of rates of evolution. First, for some traits, rate-of-divergence estimates were consistent across common gardens. Estimates of rates of divergence for quantum efficiency of 
PSII (i.e., chlorophyll fluorescence) and pollen fertility were relatively consistent between gardens for wild and crop-wild hybrid populations. Second, some quantitative traits exhibited similar directions of evolutionary change but differed in the magnitude of the estimated divergence rates between measurement environments. For example, wild water-evolved (i.e., NR-, CU-, DR- plants collectively) populations evolved longer days to flower faster than hybrid water-evolved hybrid plants in 2015, but this difference in divergence rates was less conspicuous among genotypes in 2016. Third, due to genotype-by-environment interactions, the direction and rate of trait evolution differed significantly with respect to interactions of watering history and year of measurement, and in turn, complicated interpretations of evolutionary divergence rates for each genotype. Below, I discuss these phenomena.

\subsubsection{Selecting Traits for Simple Estimates of Rate of Evolution}

Since the environment can significantly influence the expression of phenotypic traits, canalized traits (i.e., environmentally insensitive) are better candidates for measuring evolutionary rates using the method I followed (Debat and David 2001; Xie et al. 2015). Studies that have implemented rate of evolution metrics tend to focus on traits in response to a single cause of selection (e.g., Merilä et al. 2001; Campbell et al. 2009b; Mathys and Lockwood 2009; Santos et al. 2013; Presotto et al. 2016) but I have not encountered any published studies that account for environmentally sensitive phenotypic variation. Some crop traits (e.g., loss of seed shattering or metabolic processes; (Purugganan and Fuller 2011; Alseekh et al. 2017) are often environmentally insensitive and thus may be excellent candidate traits for estimating evolutionary rates. Similarly, genetically engineered resistance to herbivory or herbicides traits (Snow et al. 2010; Beckie et al. 2013; Wiersma et al. 2015) may also be appropriate candidates. Finally, studies that track the frequency of molecular markers within populations through time can provide estimates of evolutionary rates void of environmental sensitive expression, as well (e.g., Parker et al. 1998; Sørensen et al. 2007; Dlugosch and Parker 2008; Rose et al. 2009; Snow et al. 2010; Rubio-Meléndez et al. 2018).

\subsubsection{Phenotypic Plasticity Can Alter the Apparent Rate of Evolution}

Although it is well accepted that trait plasticity allows organisms to dynamically respond to changing environments, it appears as though studies that measure rate of evolution have yet to 
incorporate the influence of plasticity in their estimates. In fact, the ecological context under which plants are measured can have a substantial influence on evolutionary metrics and reduce the certainty of conclusions about evolutionary rates. My results suggest phenotypic plasticity in wild plants, and less so in hybrid plants, in response to environmental variation between common gardens, can alter apparent evolutionary divergence rates. Further, in environments with sufficient resources and particularly water availability, plants can allocate resources into extended growth periods (Funk 2013), but in stressful years plants pre-conditioned to withstand stressful, water-limited environments, like NR-evolved plants, may have a competitive advantage with selection for earlier emergence and flowering times (Franks et al. 2007; Anderson et al. 2011) to maximize the period for reproduction. Due to the interaction between a plant's genotype and its current growth environment (Franks 2011; Kooyers 2015), and potentially the epigenetic effect of the maternal environment (Wolf and Wade 2009; Germain et al. 2013; Campbell et al. 2015), discrepancies in rates between common garden years can arise and conclusions in evolutionary trajectories are intangible and unresolved.

Trait plasticity can also apparently influence the magnitude of evolution. Traits like flowering time (e.g., Franks et al. 2007), body size among birds (e.g., Santos et al. 2013), or predatory protection in invertebrates (e.g., Rabus et al. 2013) may be largely genetically determined but still be partly influenced by environmental context (see SI - Appendix 3). In a foundational paper on plasticity, researchers Falconer (1952) and Via and Lande (1985) correctly argue that a trait expressed in two environments should be measured as two separate traits. For example, leaf length on plants grown in two different environments should be analysed as two separate models. Additionally, they argue that trait responses expressed in the two environments can be genetically correlated, such that a genetic correlation of \pm 1 implies the trait is genetically identical (i.e., the same set of alleles influences trait expression. In contrast, a correlation between -1 and +1 , either (1) the influences the same set of alleles differently or (2) the trait is influenced by a different set of alleles (Falconer 1952; Via and Lande 1985). Considering my analysis of correct versus incorrect evolutionary rate estimates (section 3.3.6, paragraph 3), I found that trait correlations are not genetically identical (i.e., not perfectly correlated) and may be expressed differently in response to the environment or controlled by a different set of alleles. This further validates the confounding effect plasticity has on evolutionary rate estimates and the importance of selecting the appropriate trait (see section 3.5.4). Generally, if plant phenotypes 
are, at least partly, environmentally sensitive, traditional evolutionary rate metrics will vary between measurement environments and conclusions of the speed of plant and animal evolution may be incorrect.

Crop-wild hybrids in my experiment had similar phenotypic responses and rates across common gardens irrespective of their water selection history (Figs. 3.5-3.7). Traits in crop populations are sometimes directionally selected to have reduced environmental sensitivity (but see Sadras et al. 2009) and produce a specific phenotype in response to a particular environment or a consistent response in variable environments (Nicotra et al. 2010). To achieve these standards, breeders limit the phenotypic response by breeding for allelic homozygosity (either through dominant or recessive alleles), depending on the trait (seed size, flowering time) (Nicotra et al. 2010; Snow et al. 2010; Flint-Garcia 2013); these traits, therefore, may share the same set of alleles that respond similarly across environments (i.e., genetically identical and correlated; Via and Lande 1985). Introgression of crop traits into crop-wild hybrid populations, may explain the lack of phenotypic plasticity and minimal differences in evolutionary rate estimates in my hybrid plants across years. Thus, evolutionary metrics may prove useful in measuring the speed of invasion of crop traits into crop-wild hybrid populations.

\subsubsection{The Complexity of Measuring Rates of Evolution in Quantitative Traits: Moving Forward}

Measuring the rate of evolution of phenotypic traits (vs. molecular markers) in non-crop populations is desirable for a variety of reasons, one of which is to better comprehend the potential for plant populations to respond to environmental variation, as I have done here. Considering this, I propose several alternative approaches for measuring evolutionary rates in biological populations. At minimum, to increase reliability of estimates (Mitchell-Olds and Rutledge 1986; Conner et al. 2003), rate of evolution studies should estimate rates for traits with known selection values or heritability values (Conner et al. 2003; Mathys and Lockwood 2009). Furthermore, measuring plants in a range of common garden environments to account for phenotypic plasticity in traits will allow biologists to present evolutionary rates as a range, rather than an absolute value.

Pre-existing knowledge about a trait and its directional selection in response to an environmental pressure may help provide a better evolutionary rate estimate. If, for example, researchers are able to predict the direction of a trait in response to an environment (see chapter 
5; seedling emergence), then this, at least partly, can control the effect environmental variation has on evolutionary rate estimates. However, the problem of phenotypic plasticity still remains depending the trait's strength of selection. To be more precise in estimates of evolutionary rates, researchers should replace $V_{P}$ with $V_{G}$ in rate of evolution metrics to create more accurate estimates (Cahaner and Hillel 1980; Hartl and Clark 2007). Partitioning genetic variance out of total phenotypic variance would involve planting siblings from multiple families and for multiple populations within and across measurement environments to estimate genetic variance.

Accounting for this environmental variance may occur using analysis of variance or other partitioning methods that run the residuals of a model void of the environmental variability (i.e., residuals of a model that runs only environment in a model). Alternatively or in addition, the analyst can subtract the environmental variance, from the variance of the whole population $\left(V_{P}\right)$ to calculate the contributing genetic variance (see Dlugosch and Parker 2008; (Mitchell-Olds and Rutledge 1986; Conner and Hartl 2004) which would replace $V_{P}$ within Equation 1, such that:

$$
\text { haldanes }(h)=\frac{\left[\left(\frac{\ln \bar{V}_{G 2}}{s_{p}}\right)-\left(\frac{\ln \bar{V}_{G 1}}{s_{p}}\right)\right]}{t_{2}-t_{1}} \quad \text { (Equation 2) }
$$

Of course, genetic variance for a given trait will vary among populations and would require a rather large sample size to capture variability and calculate accurate estimates, thus implementing this method would be a more laborious approach. Additionally, an unstated assumption of my dataset is that control populations themselves are not evolving; which is likely incorrect. I attempted to correct for this by measuring the CS-CU population comparison; however, this does not fully correct the problem. Future studies should consider common gardens of seeds from ancestral populations (e.g., at the start of experiment) and current populations (i.e., $\mathrm{G}_{5}$ ) to see how far populations have deviated from ancestral conditions. Finally, I propose molecular traits (tracked using molecular markers) are also an acceptable alternative to the use $V_{G}$ of quantitative traits in estimates of evolutionary rates. Estimates of evolution at molecular marker loci are common. For example, Rose et al. (2009) tracked frequencies of wild- and crop-specific alleles in Brassica spp. in response to competition, and Sorenson et al. (2007) measured how genetic diversity changed in hybrid populations of chicory (Chichorium intybus) over two years. Molecular estimates can also be found for animal taxa; Melendez et al. (2018) tracked insecticide-resistance allele frequencies in the green peach aphid 
(Myzus persicae) in several peach orchards. Similarly, Santos et al. 2013, have quantified several microsatellite loci between introduced and native ranges of the dunnock bird (Prunella modularis). Although these studies have not calculated evolutionary rates, this calculation could be done easily post-hoc. Notably, these studies are generally limited to calculations in units of darwins rather than haldanes because molecular estimates do not usually present error estimates (i.e., standard error, standard deviation). Using Snow et al. (2010) data as an example, I calculated the rate of evolution of several crop-specific molecular markers across four populations of Raphanus raphanistrum monitored over a ten-year period (Appendix 2). Crop allele introgression proceeded as slowly as 0 darwins, indicating no change in the crop allele marker phosphoglucomutase enzyme, and as fast as $-281.3 \times 10^{3}$ darwins in the loss of white flower petal alleles from crop-wild hybrid radish populations. Studies that document environmental canalization or track molecular markers are likely the easiest with which to accurately estimate evolutionary or divergence rates. In instances where these markers are unavailable or not of interest, using genetic variance estimates may prove useful and more accurate than historic approaches. 


\subsection{Table List}

Table 3.1: Mixed model ANOVA of mean trait plasticity of) days to emergence and $b$ ) days to flowering between years in response to genotype (wild versus hybrid), watering history (WH: no rain, control sheltered, control unsheltered, and double rain), and their interaction as fixed factors, with Block (20 levels) as a random factor. Considering the non-orthogonality of the data, type III ANOVA results are presented in the table using a Kenward-Roger's adjustment for computing the degrees of freedom. The fixed effects models compute the F-statistic using the mean square error of each model (presented as Error in the table). Additionally, for each trait a $\chi^{2}$ significance test was run comparing the model with and without the block factor (i.e, measuring the significance of block in the model). (a) The model measuring days to emergence had a significant block effect $\left[\chi_{(\mathrm{df}=1)}^{2}=43.50, \mathrm{p}<0.001\right]$. (b) The model measuring days to flowering had a significant block effect $\left[\chi_{(\mathrm{df}=1)}^{2}=27.54, \mathrm{p}<0.001\right]$. Analyses were run in R-Studio (version 1.0.143) and SAS Enterprise Guide 61 where all response variables fitted to a normal distribution and an identity link function. F-statistics in the fixed effect ANOVA table are presented to indicate significant differences: $\mathrm{ns}, \mathrm{P}>0.10 ;+, \mathrm{P}<0.10 ;{ }^{*}, \mathrm{P}<0.05 ; * *, \mathrm{P}<0.01$; $* * *, \mathrm{P}<0.001$.

\begin{tabular}{lccc}
\hline $\begin{array}{c}\text { Response \& } \\
\text { Parameter }\end{array}$ & df & Mean Square & F Statistic \\
\hline a) Days to Emergence (Box-cox transformation $\lambda=$ & $-1.037937, \mathrm{n}=650)$ & \\
\hline Genotype (G) & 1 & $9.62 \times 10^{-3}$ & $9.05^{* *}$ \\
Watering History & 3 & $2.55 \times 10^{-3}$ & $2.40^{+}$ \\
(WH) & & & \\
Year (Y) & 1 & $1.30 \times 10^{-3}$ & $1.23^{\text {ns }}$ \\
WH $\times$ G & 3 & $5.80 \times 10^{-3}$ & $5.46^{* *}$ \\
WH $\times$ Y & 3 & $4.05 \times 10^{-3}$ & $3.81^{*}$ \\
G $\times$ Y & 1 & $7.00 \times 10^{-6}$ & $0.01^{\text {ns }}$ \\
WH $\times$ G $\times$ Y & 3 & $1.25 \times 10^{-3}$ & $1.17^{\text {ns }}$ \\
Error & 616 & $1.06 \times 10^{-3}$ & - \\
\hline b) Days to Flower (Box-Cox transformation $\lambda=-2.013029, n=650)$ & $36.66^{* * *}$ \\
\hline G & 1 & $3.40 \times 10^{-7}$ & $6.43^{* *}$ \\
WH & 3 & $5.96 \times 10^{-8}$ & $10.13^{*}$ \\
Y & 1 & $9.39 \times 10^{-8}$ & $8.37^{* * *}$ \\
WH $\times$ G & 3 & $7.76 \times 10^{-8}$ & $0.08^{\text {ns }}$ \\
WH $\times$ Y & 3 & $7.90 \times 10^{-110}$ & $18.62^{* * *}$ \\
G $\times$ Y & 1 & $1.73 \times 10^{-7}$ & $13.77^{* * *}$ \\
WH $\times$ G $\times$ Y & 3 & $1.28 \times 10^{-7}$ & - \\
Error & 617 & $9.28 \times 10^{-9}$ & \\
\hline
\end{tabular}


Table 3.2: Mixed model ANOVA of phenotypic differences for a) frequency of white-flowered plants, b) pollen fertility, and c) chlorophyll fluorescence between years. Considering the nonorthogonality of the data, type III ANOVA results are presented in the table using a KenwardRoger's adjustment for computing the degrees of freedom. The fixed effects models compute the F-statistic using the mean square error of each model (presented as Error in the table).

Additionally, a $\chi^{2}$ significance test was run comparing the model with and without the block factor (i.e, measuring the significance of block in the model). (a) White colour frequency was tested in response to watering history (WH: no rain, control sheltered, control unsheltered, and double rain), year $(2015,2016)$, and their interaction as fixed factors, with block (levels $=20)$ as a random factor. The model did not have a significant block effect [ $\left.\chi_{(\mathrm{df}=1)}^{2}=9.09 \times 10^{-13}, \mathrm{p}=1\right]$. (b) Pollen fertility was tested in response to genotype (G: wild, hybrid), watering history, year, and their interactions as fixed factors, with block (levels $=20)$ as a random factor. The model had a significant block effect $\left[\chi_{(\mathrm{df}=1)}^{2}=56.61, \mathrm{p}<0.001\right]$. (c) Chlorophyll fluorescence was tested in response to genotype, watering history, year, and their interactions as fixed factors, with block (levels $=20)$ as a random factor. The model did not have a significant block effect $\left[\chi_{(\mathrm{d} f=1)}^{2}=4.55 \times 10^{-13}, \mathrm{p}=1\right]$. Analyses were run in R-Studio (version 1.0.143) and SAS Enterprise Guide 61 where all response variables were fitted to a normal distribution and an identity link function. F-statistics in the fixed effect ANOVA table are presented to indicate significant differences: $\mathrm{ns}, \mathrm{P}>0.10 ;+, \mathrm{P}<0.10 ; *, \mathrm{P}<0.05 ; * *, \mathrm{P}<0.01 ; * * *, \mathrm{P}<0.001$.

\begin{tabular}{|c|c|c|c|}
\hline $\begin{array}{c}\text { Response \& } \\
\text { Parameter } \\
\end{array}$ & df & Mean Square & F Statistic \\
\hline \multicolumn{4}{|c|}{ a) White Flower Colour Frequency (Box-cox transformation $\lambda=0.6506916, n=343$ ) } \\
\hline $\begin{array}{l}\text { Watering History } \\
\text { (WH) }\end{array}$ & 3 & 0.09 & $0.31^{\mathrm{ns}}$ \\
\hline Year $(Y)$ & 1 & 0.12 & $0.41^{\mathrm{ns}}$ \\
\hline $\mathrm{WH} \times \mathrm{Y}$ & 3 & 0.76 & $2.56^{+}$ \\
\hline Error & 317 & 0.30 & - \\
\hline \multicolumn{4}{|c|}{ b) Pollen Fertility (Box-Cox transformation $\lambda=1.767388, \mathrm{n}=1064$ ) } \\
\hline Genotype $(\mathrm{G})$ & 1 & 1.15 & $51.91^{* * *}$ \\
\hline WH & 3 & 0.02 & $0.95^{\mathrm{ns}}$ \\
\hline $\mathrm{Y}$ & 1 & 0.12 & $5.94^{*}$ \\
\hline $\mathrm{WH} \times \mathrm{G}$ & 3 & 0.01 & $0.37^{\mathrm{ns}}$ \\
\hline $\mathrm{WH} \times \mathrm{Y}$ & 3 & 0.03 & $1.55^{\mathrm{ns}}$ \\
\hline $\mathrm{G} \times \mathrm{Y}$ & 1 & 0.12 & $5.97^{*}$ \\
\hline $\mathrm{WH} \times \mathrm{G} \times \mathrm{Y}$ & 3 & 0.01 & $0.42^{\mathrm{ns}}$ \\
\hline Error & 1030 & 0.022 & - \\
\hline \multicolumn{4}{|c|}{ c) Chlorophyll Fluorescence (Box-Cox transformation $\lambda=6.90741, n=413$ ) } \\
\hline G & 3 & $5.231 .26 \times 10^{-4}$ & $6.47^{*}$ \\
\hline WH & 1 & $1.26 \times 10^{-4}$ & $1.56^{\mathrm{ns}}$ \\
\hline Y & 1 & $4.08 \times 10^{-3}$ & $50.59^{* * *}$ \\
\hline $\mathrm{WH} \times \mathrm{G}$ & 3 & $1.21 \times 10^{-4}$ & $1.49^{\text {ns }}$ \\
\hline $\mathrm{WH} \times \mathrm{Y}$ & 3 & $1.45 \times 10^{-5}$ & $0.18^{\mathrm{ns}}$ \\
\hline $\mathrm{G} \times \mathrm{Y}$ & 1 & $6.09 \times 10^{-5}$ & $0.75^{\mathrm{ns}}$ \\
\hline $\mathrm{WH} \times \mathrm{G} \times \mathrm{Y}$ & 3 & $5.81 \times 10^{-5}$ & $0.72^{\text {ns }}$ \\
\hline Error & 379 & $8.08 \times 10^{-5}$ & - \\
\hline
\end{tabular}


Table 3.3: Repeated measures fixed-factor ANOVA of divergence rates of three traits [ a) days to emergence, b) days to flowering, c) frequency of white-flowered plants] and their response to genotype (wild versus hybrid), watering history (WH: no rain, control unsheltered, and double rain), and their interaction, with Year as the repeated measure. In models measuring (a) emergence time and (b) flowering time, 27 populations, in total, were considered when testing the between subject's effects. (c) The white flower colour frequency model considered 14 populations when testing the between subject's effects. The error term used for within subject's and between subject's effects are listed in the ANOVA tables under each respective category along with each model's sample size (n). F-statistics are presented to indicate significant differences: $\mathrm{ns}, \mathrm{P}>0.10 ;+, \mathrm{P}<0.10 ; *, \mathrm{P}<0.05 ; * *, \mathrm{P}<0.01 ; * * *, \mathrm{P}<0.001$.

\begin{tabular}{|c|c|c|c|c|c|c|c|}
\hline \multirow{2}{*}{$\begin{array}{c}\text { Response } \\
\& \\
\text { Parameter }\end{array}$} & \multicolumn{3}{|c|}{ Within Subject's Effects } & \multirow{2}{*}{$\begin{array}{c}\text { Response } \\
\& \\
\text { Parameter }\end{array}$} & \multicolumn{3}{|c|}{ Between Subject's Effects } \\
\hline & df & $\begin{array}{c}\text { Mean } \\
\text { Square }\end{array}$ & $\begin{array}{c}\mathbf{F} \\
\text { Statistic }\end{array}$ & & df & $\begin{array}{c}\text { Mean } \\
\text { Square }\end{array}$ & $\begin{array}{c}\text { F } \\
\text { Statistic }\end{array}$ \\
\hline \multicolumn{8}{|c|}{ a) Days to Emergence Divergence Rate $(n=257)$} \\
\hline Year $(Y)$ & 1 & 1.02 & $26.77^{* * *}$ & $\begin{array}{l}\text { Watering } \\
\text { History } \\
(\mathrm{WH})\end{array}$ & 2 & 0.12 & $0.69^{\mathrm{ns}}$ \\
\hline $\mathrm{Y} \times \mathrm{WH}$ & 2 & 0.24 & $6.38^{* *}$ & $\begin{array}{l}\text { Genotype } \\
\text { (G) }\end{array}$ & 1 & 2.12 & $12.06^{* * *}$ \\
\hline $\mathrm{Y} \times \mathrm{G}$ & 1 & 1.44 & $37.68^{* * *}$ & $\mathrm{WH} \times \mathrm{G}$ & 2 & 0.07 & $0.40^{\mathrm{ns}}$ \\
\hline $\begin{array}{l}\mathrm{Y} \times \mathrm{WH} \times \\
\mathrm{G}\end{array}$ & 2 & 0.02 & $0.57^{\mathrm{ns}}$ & Error & 21 & 0.18 & \\
\hline $\begin{array}{l}\text { Error } \\
\text { (Year) }\end{array}$ & 224 & 0.04 & & & & & \\
\hline \multicolumn{8}{|c|}{ b) Days to Flowering Divergence Rate $(n=257)$} \\
\hline Y & 1 & 0.69 & $10.66^{* *}$ & WH & 2 & 0.15 & $0.77^{\mathrm{ns}}$ \\
\hline $\mathrm{Y} \times \mathrm{WH}$ & 2 & 0.12 & $1.93^{\mathrm{ns}}$ & $\mathrm{G}$ & 1 & 3.82 & $6.86^{* * *}$ \\
\hline$Y \times G$ & 1 & 0.11 & $1.78^{\mathrm{ns}}$ & $\mathrm{WH} \times \mathrm{G}$ & 2 & 0.52 & $0.93^{\mathrm{ns}}$ \\
\hline $\begin{array}{l}\mathrm{Y} \times \mathrm{WH} \times \\
\mathrm{G}\end{array}$ & 2 & 0.13 & $2.06^{\mathrm{ns}}$ & Error & 21 & 0.56 & \\
\hline $\begin{array}{l}\text { Error } \\
\text { (Year) }\end{array}$ & 224 & 0.06 & & & & & \\
\hline \multicolumn{8}{|c|}{ c) White Flower Colour Frequency Divergence Rates $(n=140)$} \\
\hline Y & 1 & 1.89 & $18.35^{* * * *}$ & WH & 2 & 0.12 & $0.84^{\mathrm{ns}}$ \\
\hline $\mathrm{Y} \times \mathrm{WH}$ & 2 & 0.07 & $0.71^{\mathrm{ns}}$ & Error & 11 & 0.66 & \\
\hline $\begin{array}{l}\text { Error } \\
\text { (Year) }\end{array}$ & 123 & 0.10 & & & & & \\
\hline
\end{tabular}


Table 3.4: Correlation coefficients $(r)$ that measure the relationship between divergence rates estimated using evolved and reference populations from the same common garden (a correct estimation method) versus divergence rates estimated using evolved plants and reference populations grown in different environments (an incorrect estimation method). The common garden year refers to which evolved plant populations were used in the estimation of divergence rates. P-values estimated for each correlation coefficient are also presented.

\begin{tabular}{ccc}
\hline Trait & Year & $\begin{array}{c}\text { Correlation }(\mathbf{r}) \text { of divergence rate estimates } \\
(\mathbf{P}-\text {-value })\end{array}$ \\
\hline \multirow{2}{*}{ Emergence date } & $2015(\mathrm{n}=135)$ & $0.55(<0.001)$ \\
& $2016(\mathrm{n}=122)$ & $0.891(<0.001)$ \\
\hline \multirow{2}{*}{ Flowering date } & $2015(\mathrm{n}=134)$ & $0.110(0.207)$ \\
& $2016(\mathrm{n}=122)$ & $0.853(<0.001)$ \\
\hline \multirow{2}{*}{ Leaf Length } & $2015(\mathrm{n}=135)$ & $0.10(0.911)$ \\
& $2016(\mathrm{n}=122)$ & $0.974(<0.001)$ \\
\hline \multirow{2}{*}{ Stem diameter } & $2015(\mathrm{n}=109)$ & $-0.335(0.005)$ \\
& $2016(\mathrm{n}=122)$ & $-0.346(<0.001)$ \\
\hline
\end{tabular}




\subsection{Figure List}

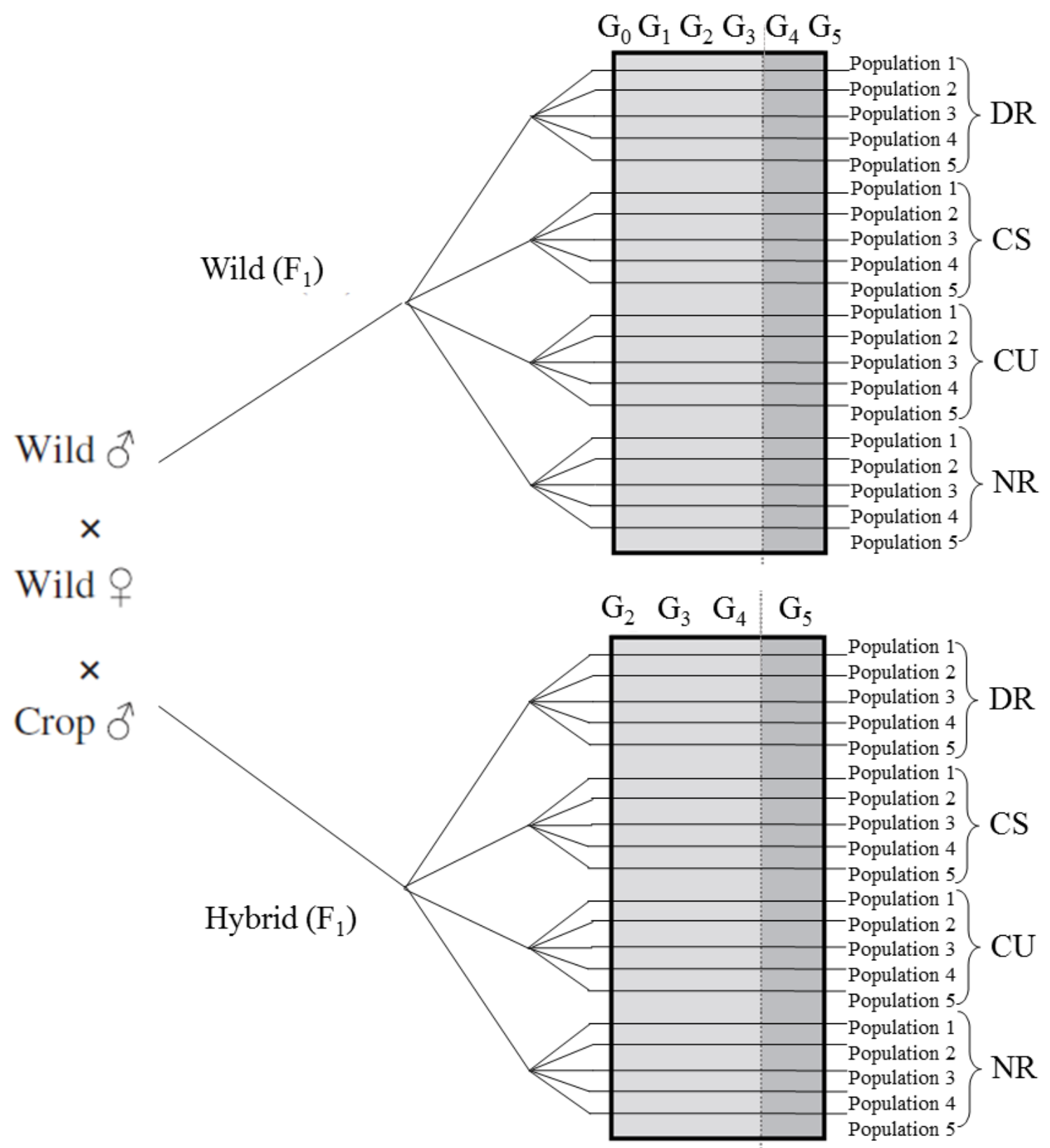

Figure 3.1: The watering history of wild and hybrid plant populations from 2010 to 2014. In 2010, crop and wild plants were planted in 36 plots as part of a randomized block design at the Waterman Farm at Ohio State University. The plots, from the parental generation $\left(\mathrm{G}_{0}\right)$, experienced one of four watering histories - double rain (DR), control sheltered (CS), control unsheltered (CU), and no rain (NR). Gene-flow naturally occurred between and among wild and cultivated-wild plants within the plots and gave rise to the first generation (i.e., $\left.\mathrm{F}_{1}\right)$ of wild and crop $\times$ wild hybrid (R. sativus $\times R$. raphanistrum) seeds. Wild and hybrid $2^{\text {nd }}$ generation $\left(\mathrm{G}_{2}\right)$ to $4^{\text {th }}$ generation $\left(\mathrm{G}_{4}\right)$ plants (5 replicate populations per watering treatment) were grown under the same watering conditions at the Koffler Scientific Reserve (KSR) in King City, Ontario, Canada. Fifth generation plants were grown in 2015 and 2016 common gardens at KSR. 
a)

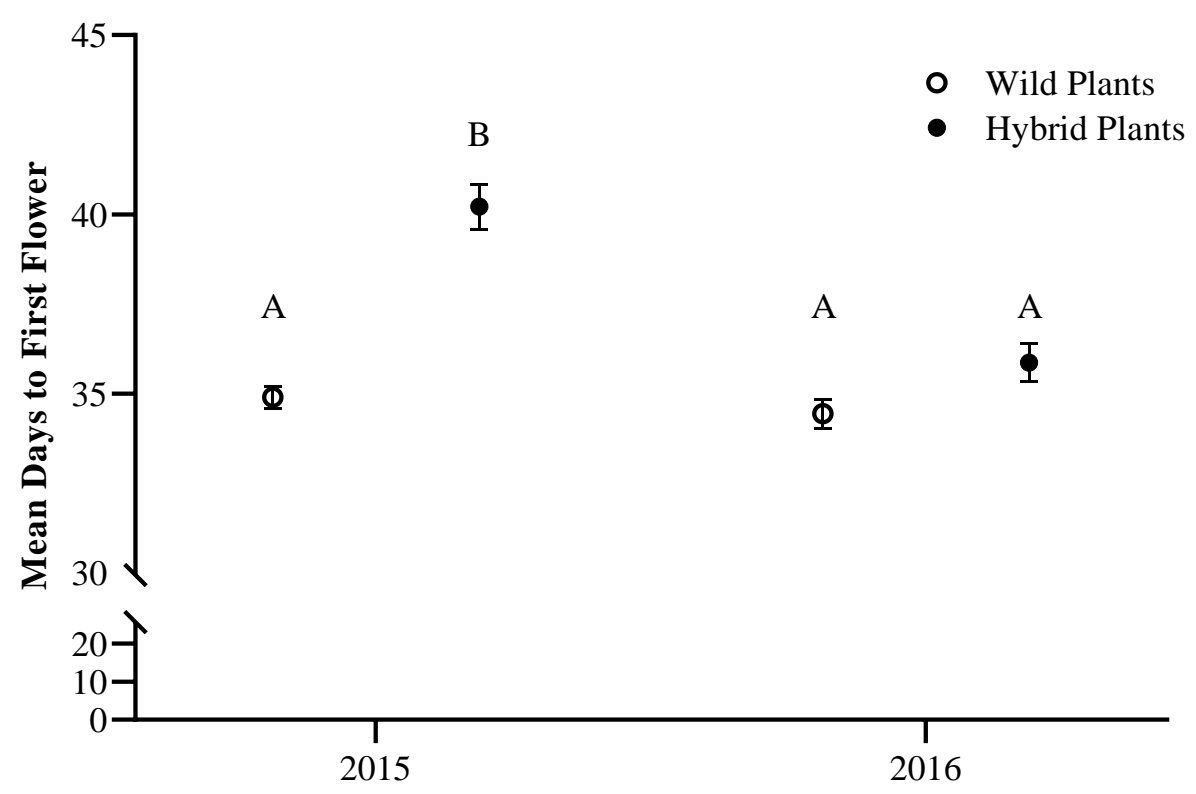

Year by Genotype

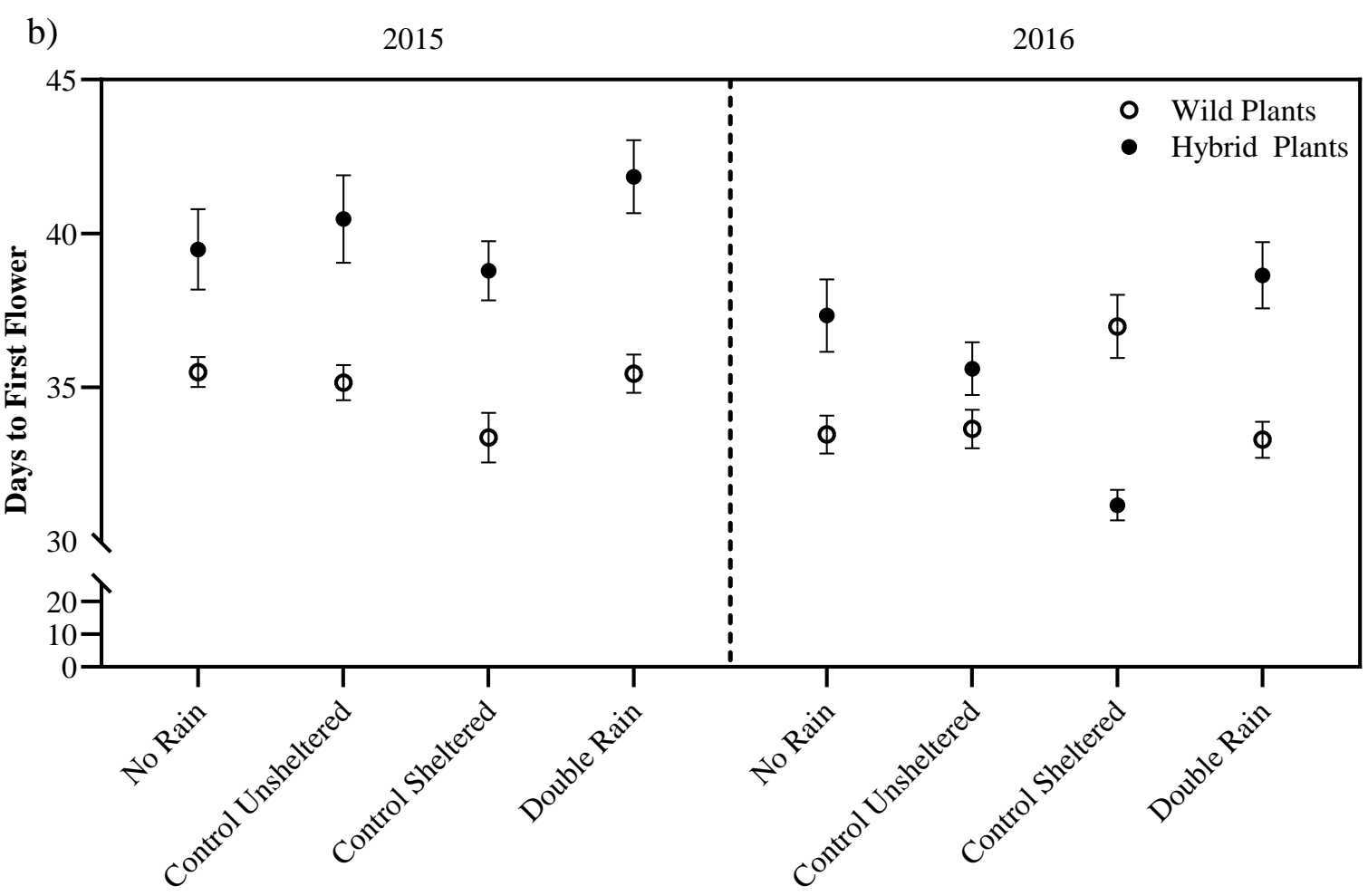

Watering History by Genotype

Figure 3.2: Comparing the evolution of flowering phenology of $\mathrm{G}_{5}$ wild and hybrid populations grown under one of three environmental conditions from $\mathrm{G}_{2}-\mathrm{G}_{4}$ and then grown in a common garden in $\mathrm{G}_{5}$. Mean days to flower $( \pm \mathrm{SE}$ ) between (a) genotype by years (wild - open circles, hybrid - closed circles) and (b) watering history by genotype by years (NR-no rain, CU-control unsheltered, CS-control sheltered, DR-double rain; wild - open circles, hybrid - closed circles). Non-shared capital letters signify statistical differences between years. 


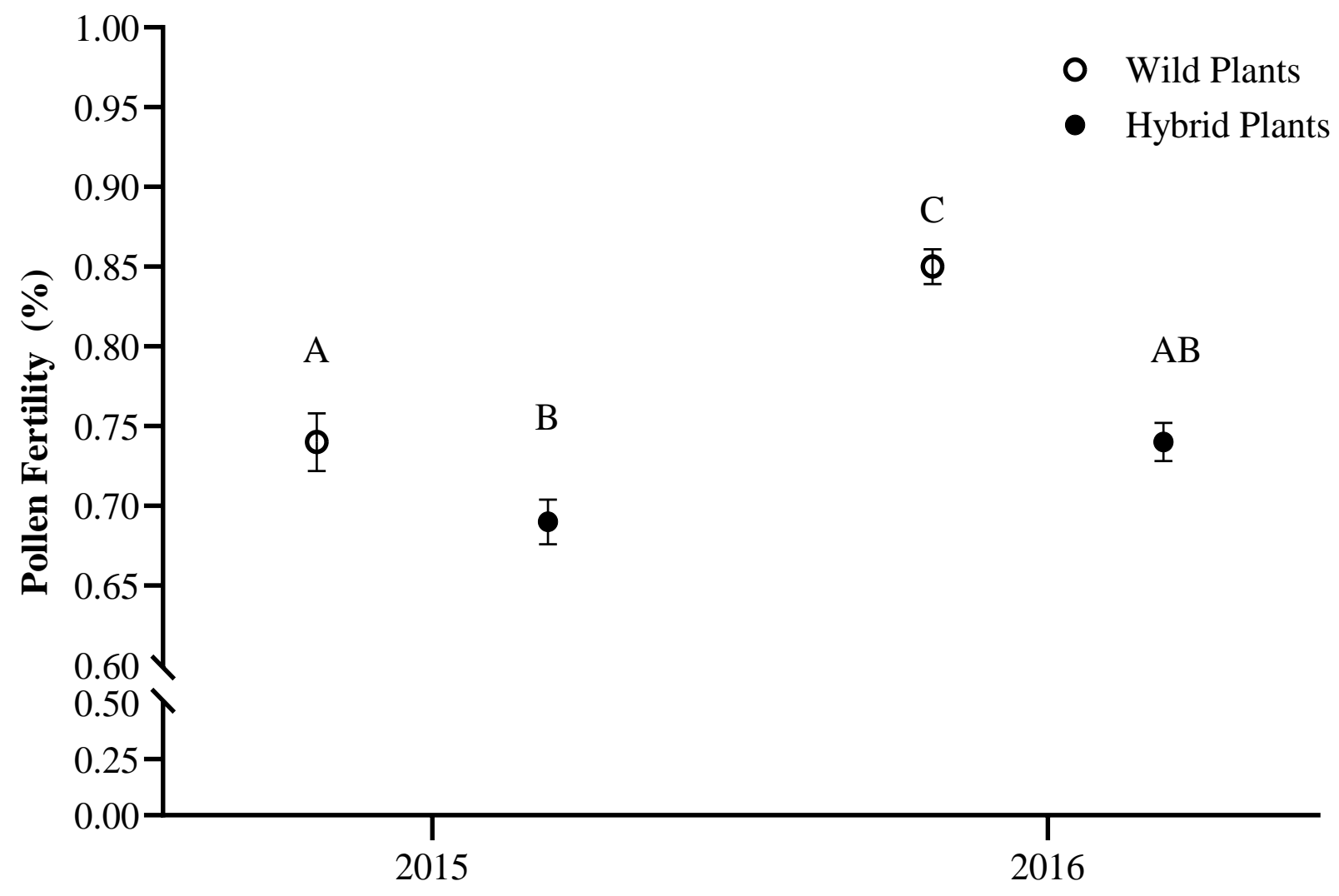

Year by Genotype

Figure 3.3: Comparing the evolution of mean pollen fertility of $\mathrm{G}_{5}$ wild and hybrid populations grown under one of three environmental conditions from $\mathrm{G}_{2}-\mathrm{G}_{4}$ and then grown in a common garden in $\mathrm{G}_{5}$. Graph displays mean percent pollen fertility $( \pm \mathrm{SE})$ between genotypes (wild open circles, hybrid - filled circles) between years. Letters above circles represent pair-wise differences, where non-shared letters signify statistical differences and shared letters represent no significant difference among treatments. 


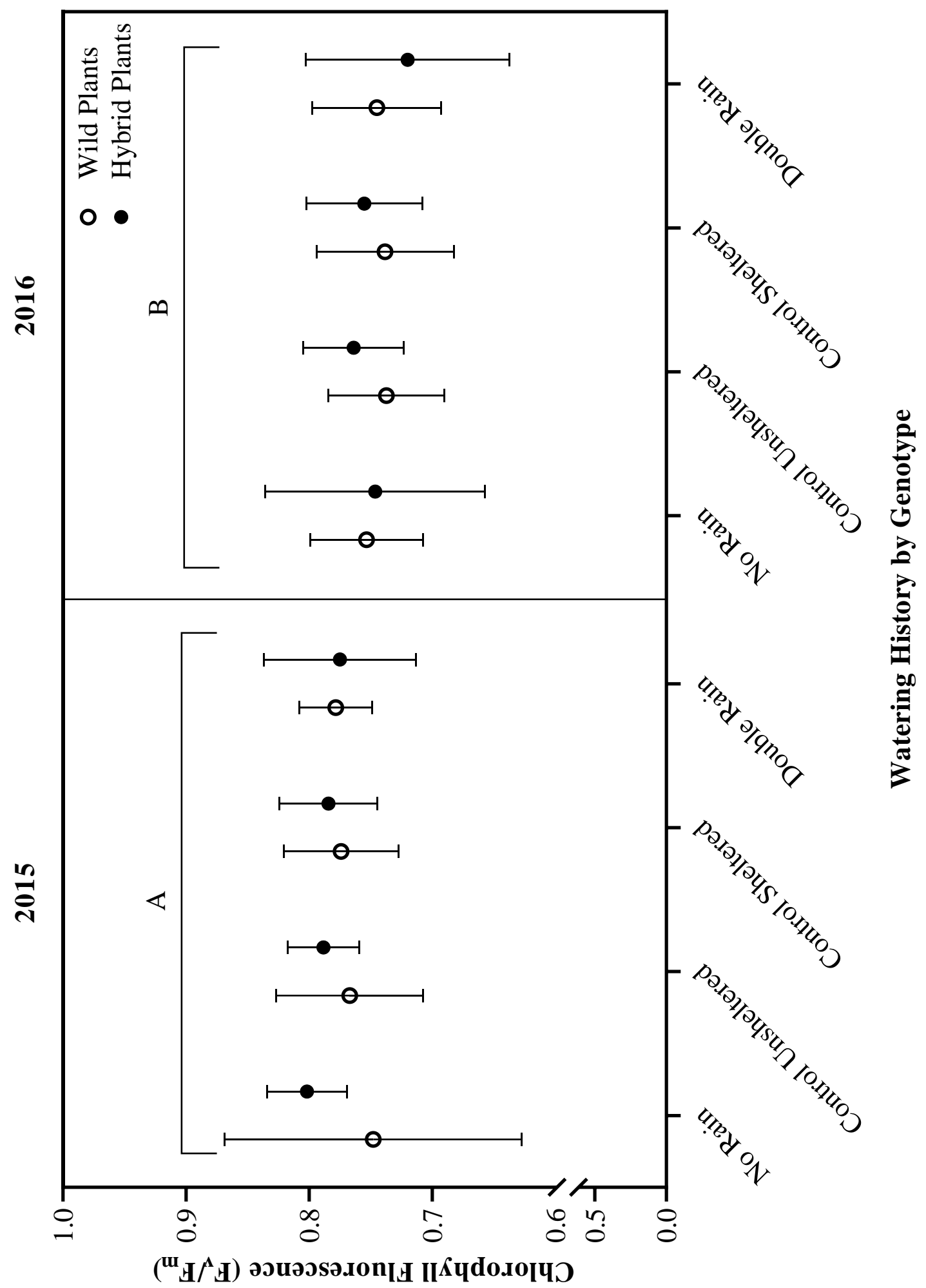

Figure 3.4: Comparing the evolution of mean quantum efficiency of PSII ( \pm SE) between G5 genotypes (wild - open circles, hybrid - filled circles) grown under one of three environmental conditions (watering histories: NR-no rain, CU-control unsheltered, CS-control sheltered, DRdouble rain) between common garden years. Non-shared capital letters signify statistical differences between years. 


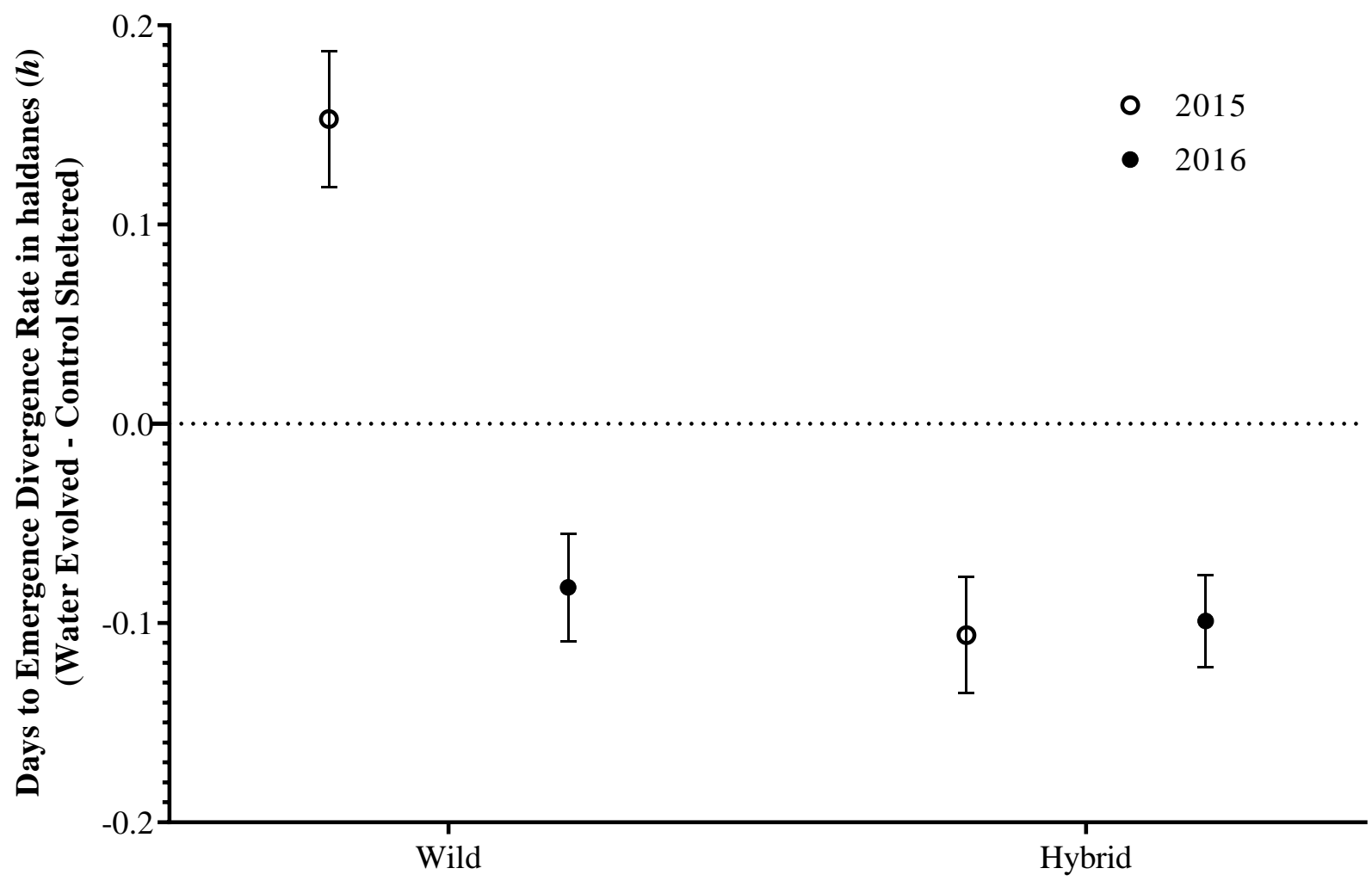

Genotype by Year

Figure 3.5: Comparing evolutionary rate estimates (presented in haldanes) of seedling emergence times ( $\pm \mathrm{SE}$ ) across genotypes (wild - open circles, hybrid - closed circles) between years. Among wild populations, values represent the change between wild water-evolved plants and wild CS plants. Similarly, among hybrid populations, values represent the change between hybrid water-evolved and CS population. 
Figure 3.6: Comparing evolutionary rate estimates of $\mathrm{G}_{5}$ wild and hybrid radish plants grown under one of three environmental conditions between common garden years. Divergence rates are presented in haldanes of (a) the frequency of white-flowered plants ( \pm SE) and (b) days to emergence $( \pm$ SE) across watering histories [no rain $(N R)$ - open circles, control unsheltered (CU) - grey circles, double rain (DR)- black circles] between years. Values represent change between water-evolved plants and control sheltered plants for each respective treatment (i.e., change between NR-evolved and control sheltered, CU-evolved and control sheltered, and DRevolved and control sheltered).

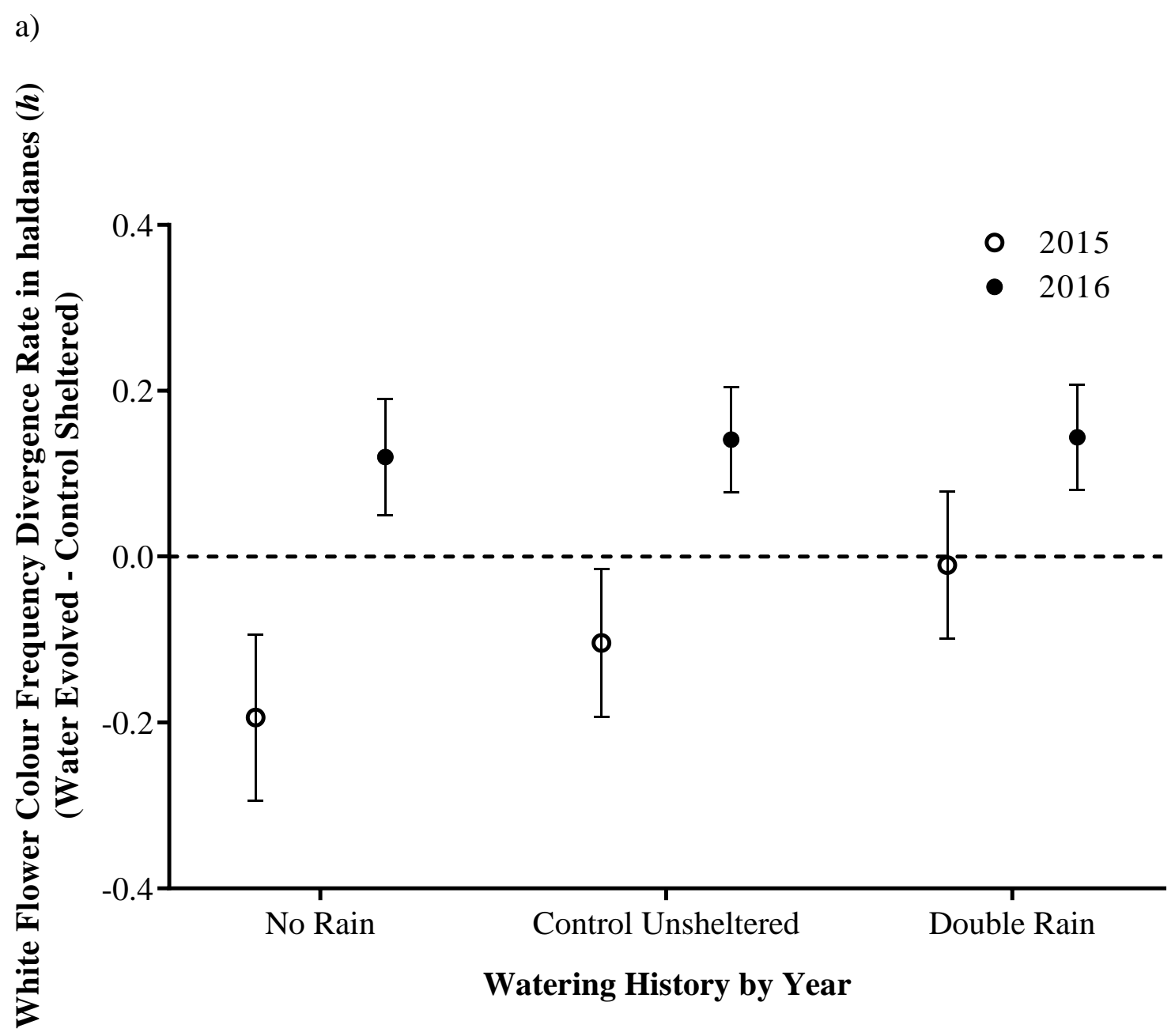




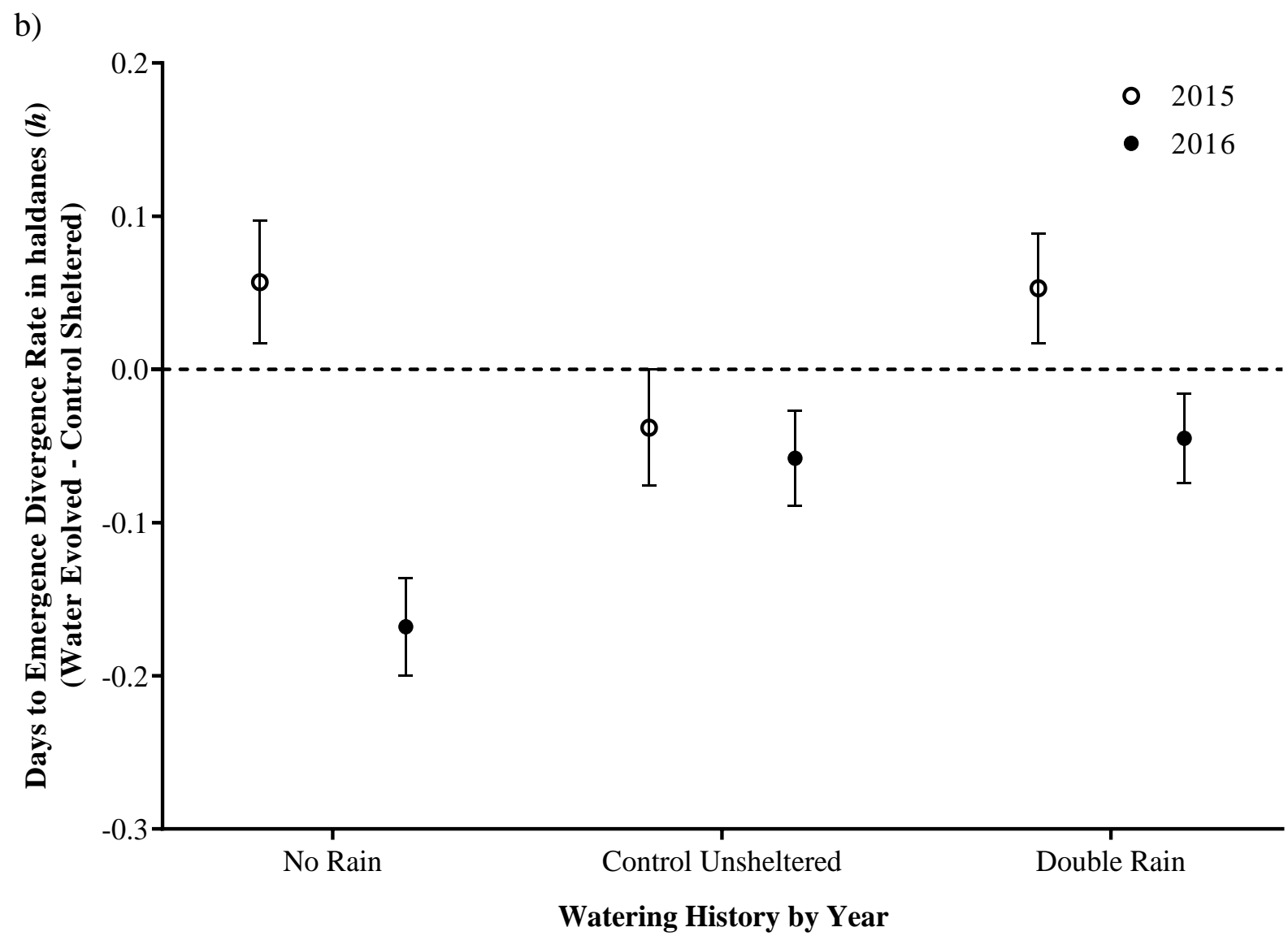




\section{Chapter 4: Variation in Fitness and Selection on Crop traits in Crop-Wild Hybrid Populations}

\subsection{Abstract}

Over the last two decades, population biologists have evaluated rates of gene flow among crops and their wild relatives to evaluate the risk that engineered genes could pose if incorporated into wild populations. Crop gene flow is generally expected to have negative fitness consequences for recipient weed populations since crop-derived traits were expected to be nonadaptive in wild and weedy populations. Yet, persistent introgression can occur and varies in intensity among locations, depending on the populations involved. Thus, I asked: are crop traits consistently selected against, regardless of environmental context? Using data collected over ten years and in three locations, I demonstrated that natural selection can intermittently favour the introgression of crop-derived phenology schedules (germination and flowering) in wild (Raphanus raphanistrum) and crop-wild hybrid populations of radish $(R$. raphanistrum $\times R$. sativus). This suggests that diverse regimes of selection or rates of gene flow among populations can create a landscape of adaptive phenologies that reflect both domesticated and nondomesticated genotypes in weedy radish populations. Thus, the idiosyncratic nature of both gene flow and selection may hinder the management and monitoring of crop gene flow but may create spatial variation in hot and lowspots of introgression. Given these results, spatial variation in selection on crop traits should be considered before making predictions about crop gene introgression.

Keywords: Crop-to-wild hybridization, germination, flowering phenology, hybridization hotspots, introgression, lowspots, natural selection, Raphanus raphanistrum, Raphanus sativus *Intended for submission to Proceedings of the National Academy of Sciences of the United States of America (PNAS - ISSN: 1091-6490) 


\subsection{Introduction}

The movement of novel (i.e., new) crop alleles into the populations of wild and/or weedy relatives begins with spontaneous hybridization with crops (Ellstrand and Hoffman 1990; Snow and Palma 1997; Ellstrand 2003). For long-term introgression to occur, semi-fertile crop-wild hybrid offspring must survive to reproduce (Anderson and Stebbins 1954; Linder et al. 1998; Jenczewski et al. 2003; Campbell et al. 2016a). However, the degree to which the relative fitness of crop-wild hybrid offspring varies spatially and temporally has been under-explored. Rather, common garden assessments of hybrid fertility often occur within a limited number of physical locations and in a limited range of seasons (Langevin et al. 1990; Snow et al. 1998; Campbell \& Snow 2007; although see Hooftman et al. 2005; Campbell et al. 2006; Snow et al. 2010). Variation in fitness is a consequence of selection acting on both reproduction and associated lifehistory traits. Therefore, variation in fitness may dramatically influence introgression rates across regions where crop and wild and/or weedy relatives co-occur (Slatkin 1987; Bartsch et al. 1999; Papa and Gepts 2003; Tesso et al. 2008; Mutegi et al. 2015). Although gene flow rates are well documented and important for predicting novel crop trait escape, the consistency of the hybrid fitness and thus selection on crop traits across ecological contexts remains under-studied.

Crop traits often have a range of fitness consequences for plants in ruderal populations (Ellstrand et al. 2010). Many crop traits are maladaptive, or correlated with a maladaptive trait, in weedy populations, thereby potentially mitigating the evolution of more aggressive weeds through ferality (via de-domestication) or gene-escape (via gene flow); these include traits like altered phenology, increased investment in vegetative (vs reproductive) characteristics, and reduced dispersal (e.g., Baker et al.1965; Baker 1974; Doebley 1992; Harlan 1992). For instance, crop breeding strongly selects for rapid and synchronous seed germination, whereas it is more difficult to eradicate weeds that exhibit variability in germination dates (Baker et al. 1965; Baker 1974; Doebley and Stec 1991; Doebley 1992). In wild populations, early seedling emergence allows weeds the time to accrue added biomass, and thus increase reproductive success, when biomass is correlated with seed production. In contrast to crop populations that are often selected to delay flowering and increase relative investment in large vegetative traits, weed populations often flower early (e.g., Baack et al. 2008; Campbell \& Snow 2009), because early flowering allows weeds to complete their life cycle before spring tilling or the end of the growing season 
(Baker et al. 1965; Baker 1974; Snow and Campbell 2005). More subtly, early seedling emergence acquired from crop plants may facilitate reproduction in crop-wild hybrid plants that have retained the delayed flowering crop trait. Moreover, indehiscent fruit (which remain attached to the plant) and easily shattering fruit may prove to be disadvantageous traits in weedy populations (Gressel and Al-Ahmad 2012; Heredia and Ellstrand 2014), by reducing dispersal and increasing herbivory opportunities, respectively. Yet, crops are generally more resistant to consumption than their wild relatives (Turcotte et al. 2014) and crop traits associated with GE traits, such as herbicide (e.g., glufosinate; Mikkelsen et al. 1996) or insecticide resistance (Bt gene) can be adaptive to weed populations growing in agricultural environments (Kareiva et al. 1994; Snow et al. 1999; Snow et al. 2003; Ashworth et al. 2016). Thus, crop traits in response to “obvious" selection pressures, such as herbicide resistance, may persist more frequently in weedy/wild populations if they are not costly to produce and maintain (Whitton et al. 1997; Snow et al. 1999; Snow et al. 2003; Ellstrand et al. 2013). However, it remains unknown whether selection favours crop derived traits and remains untested whether crop-derived life-history traits (e.g., emergence and flowering time) will persist across diverse environmental contexts.

The relative advantage of a particular life-history strategy may be context dependent (Ellstrand et al. 2010; Anderson et al. 2011; Campbell et al. 2016c) and thus crop traits may experience a range of both positive and negative selection gradients across a hybrid zone (Barton and Hewitt 1985; Barton 2001; Todesco et al. 2016). For example, Campbell et al. (2006) found that crop-wild hybridization in radish resulted increased fecundity of crop-wild hybrid radish plants compared to wild plants when grown in California but not Michigan. Similarly, populations of crop-wild hybrid Helianthus (i.e., sunflower) experienced stronger selection for larger leaf area, a crop-derived trait, in Indiana than Nebraska (Baack et al. 2008) and this is a consistent pattern in the literature where selection favours larger, crop-derived phenotypes in wild populations (Dechaine et al. 2009; Kost et al. 2015). Despite the potentially profound implications of crop alleles escaping into weed populations (Ellstrand et al. 2010), studies demonstrating the context dependency of phenotypic selection on crop traits in weed populations are relatively rare (but see Baack et al. 2008; Dechaine et al. 2009; Owart et al. 2014; Kost et al. 2015). 


\subsubsection{Objectives}

After more than a decade of replicated experiments of gene flow from crop radish cultivars to wild radish populations across North America (total $n=12,657$ plants measured), I have amassed a sizeable dataset describing the evolution of crop traits in weed populations (where crop-wild hybrid and feral crop populations are compared wild populations) and the relative fitness of crop-wild hybrid plants compared to their parental taxa. Here I evaluated whether selection could account for the evolution of crop-derived phenology expressed in cropwild hybrid radish (Raphanus raphanistrum $\times R$. sativus) and feral radish ( $R$. sativus) populations, relative to wild radish ( $R$. raphanistrum) populations that lack crop-derived traits, grown over a 12-year period across four North American locations (Michigan, Ontario, Ohio, Texas). First, I characterized seedling emergence and flowering phenology in each genotype (hybrid, wild, and crop), population, and environment. Then, I estimated the direction and strength of selection exerted on wild, crop-wild hybrid, and feral plant populations using a Lande-Arnold approach (Lande and Arnold 1983) in an attempt to determine:

(1) Are differences in selection gradients between genotypes?

(2) Are crop traits are consistently selected against in all tested locations?

If the wild phenotype is selectively advantageous, then crop-wild hybrid plants will experience selection for variable emergence times and earlier flowering time - phenologies representative of wild plants - across geographical landscapes. If crop traits are selectively advantageous, hybrid plant populations may experience directional selection towards early emergence and later flowering. If, however, selection for wild or crop phenologies may vary with ecological context and will result in introgression hot- and low-spots across the hybrid zone. 


\subsection{Methods}

\subsubsection{Study System}

Raphanus sativus (radish), is an open-pollinated, self-incompatible crop often selected for large, colourful roots, synchronous early germination, delayed flowering and high levels of seed production (Snow and Campbell 2005). The wild relative of crop radish, Raphanus raphanistrum, is a self-incompatible, hermaphroditic annual that has recently colonized North America, and is commonly found in nutrient-rich, frequently disturbed areas including agricultural fields and coastal dune ecosystems (Holm 1997). This species tends to exhibit asynchronous seed germination, early flowering, and high levels of seed production (Snow and Campbell 2005). Flower petal colour frequencies differ between species and has been used repeatedly as a crop-specific marker in these populations (e.g., Snow et al. 2001; Campbell et al. 2006; Chapter 2). White, pink, or purple flowers are most common in Raphanus sativus populations whereas yellow flowers or more rarely, white, pink or bronze flowers are characteristic of $R$. raphanistrum (Panetsos and Baker 1967; Kay 1976; Conner et al. 1996b). Yellow carotenoid pigment is recessive to white petal colour (Panetsos \& Baker 1967). The genetic basis of pink hues is more complex (Stanton 1987), so this trait was not used as a genetic marker in any of my studies.

\subsubsection{Seed Sources for Experimental Populations}

\subsubsection{Michigan and Texas Plants}

As in Campbell et al. (2006), Campbell and Snow (2007), Campbell and Snow (2009), Campbell et al. (2009a); Campbell et al. (2009b), Campbell et al. (2014), Hovick and Whitney (2014), and Campbell et al. (2016c), seeds from several-hundred plants were collected from a natural population of wild $R$. raphanistrum in an agricultural field in Pellston, MI, USA in fall 2001. In a glasshouse at Ohio State University (Columbus, USA), 100 wild plants were germinated, grown, and hand-pollinated with either wild pollen to create $F_{1}$ wild genotypes or crop pollen to create $\mathrm{F}_{1}$ hybrid genotypes. Crop pollen was obtained from 100 'Red silk' $R$. sativus plants (Harris-Moran Seed Co., Modesto, CA, USA), a common, contemporary variety. Below, I refer to radish genotypes as 'wild' or 'hybrid' based on hybridization in the $\mathrm{F}_{1}$ offspring of a crop-to-wild mating event. In fact, only one publication used plants from diverse crop 
cultivars and wild populations (Snow et al. 2010) and demonstrated no significant difference among crop-wild hybrid plants from these diverse crosses in terms of reproductive success. Plants generated in Snow et al. (2010) used wild plants from Pellston, MI, Deer Isle, ME, Leesburg, GA and Binghamton, NY and four crop cultivars (Cabernet, Cherriette, Red Silk and Scarlet Globe). The $\mathrm{F}_{1}$ generation of these plants were subsequently exposed to several generations of natural selection.

\subsubsection{Ontario Plants}

As in Campbell et al. (2015), (Teitel et al. 2016a), and Chapter 2, seeds were haphazardly collected by selecting fruit from 60 plants with yellow flower color (a homozygous recessive trait) across three field populations near Binghamton, NY, USA (Conner and Via 1993). From this collection, a population (>200 plants) was grown for several generations in a greenhouse in East Lansing, MI USA. The crop radish cultivar (Red Silk, Harris-Moran Seed Co., Modesto, CA, USA) was homozygous for white flower colour, and the $R$. raphanistrum population was homozygous for yellow flower color. This genetic marker allowed us to distinguish among $\mathrm{F}_{1}$ wild and $F_{1}$ crop-wild hybrid plants and to confirm cross type in this experiment. In agricultural fields on the Waterman Experimental Farm at the Ohio State University (Columbus, USA), 36 plots (2.4 m x $3.0 \mathrm{~m}$, each) arranged into nine blocks were planted with R. sativus $(\mathrm{n}=324)$ and R. raphanistrum $(n=324)$. Four watering treatments (control unsheltered, control sheltered, no rain, and double rain treatments described below) were randomly applied to each plot within each block.

(1) Control Unsheltered (CU): To establish a control precipitation treatment, ambient rainwater fell on un-manipulated plots.

(2) Control Sheltered (CS): To determine the effect of the rain-out shelter on plant growth, ambient rainwater, collected from the shelter, was applied to the plot.

(3) No rain (NR): To create relatively dry soil conditions, water collected from NR shelter barrels was withheld.

(4) Double Rain (DR): To create relatively wet soil conditions, water collected from DR and NR shelters was applied to DR plots; that is, double the ambient rainfall. 
These plots created the first generation $\left(\mathrm{F}_{1}\right)$ hybrid genotypes used in subsequent Ontario experiments. Similar to Michigan and Texas plants, the $F_{1}$ generation of these plants were exposed to several generations of natural selection in each of their respective watering treatments.

\subsubsection{Data Sets}

Data sets were limited to those for which I possessed individual-based data on wild, hybrid, crop, and/or feral Raphanus. Detailed descriptions of the common gardens in which these genotypes were grown are available in each publication listed in Appendix 6. Briefly below, I also compare and contrast the experimental conditions under which plants were grown. As the reader will note, plants were grown in a diversity of containers (e.g., plastic pots, aluminum pans, or in-ground field conditions), under a diversity of competitive conditions (e.g., intra- vs. interspecific competition at a range of densities), in a diversity of geographic locations (Michigan, Texas, Ontario, Ohio), including a diversity of genotypes, and across a range of 12 growing seasons. My requirements for the inclusion of studies included (i) availability of planting date, seedling emergence date, and flowering date and (ii) a fecundity estimate (i.e., number of seeds per plant), and (ii) an estimate of size (i.e., leaf length, stem diameter, or biomass). Although my overall question concerns the differences between genotypes across locations, it is imperative to note that within each study, individuals of a particular genotype within a given experimental treatment (described in Appendix 6-Table A6.1) were considered distinct populations. After applying my inclusion criteria, I had a sample of 7348 plants representing 44 wild populations, 39 hybrid populations, and eight crop populations. Considering the relatively low number of crop populations available, my life-history and phenotypic selection analyses primarily focused on wild and hybrid genotypes. Similarly, Texas and Ohio lacked replication across years and were also omitted from the analyses. However, I considered the selection experienced by these additional populations in the discussion.

\subsubsection{General Method of Measuring Date of Emergence, Flowering, and Seed Set}

Across each experimental year and location, once planted, seeds at each site were monitored to record the date of seedling emergence from the soil and the date of when the first flower opened. From these measurements, emergence time (i.e., the number of days between 
planting and seedling emergence) and the age at which a plant flowered (i.e., the number of days between emergence and the first flower) was calculated. Stem diameter and longest leaf length were additional life-history traits measured during first flowering across most studies and are typical parental markers that can be used as indices of plant size since they are highly correlated and heritable (Campbell and Snow 2007). Measurements were made until plant senescence, where flowering concluded and fruits ripened, at which point plants were harvested. Additionally, a random sample of 10 fruits were collected, counted, and averaged from each plant to get an estimate of average seeds per fruit; this value was multiplied by the number of fruits produced per plant to get an estimate of the total number of seeds produced per plant. Finally, the above-ground biomass, an additional index of plant size growth, was measured for each plant void of any fruits or leaves. I used leaf length as the main growth correlate when calculating selection on traits, however when leaf length was not available, I used stem diameter or biomass (see Appendix 6- Table A6.1 for a detailed account of growth correlates used for each study).

\subsubsection{Statistical Analysis}

\subsubsection{Life-History Trait Response}

To test for differences in average days to emergence, days to first flower, and number of seeds per plant between wild and hybrid populations grown across multiple locations and years, I ran nested, mixed-model MANCOVA. Genotype and year nested in location were run as the main factors and generation number post-hybridization as the covariate (as in Galen et al. 1987; Caruso 2000; Campbell et al. 2009a). To determine if the phenotypic crop-derived marker, frequency of white flowered plants, predicted days to emergence, days to first flower, and number of seeds per plant, I ran a mixed-model MANCOVA number of seeds per plant on hybrid populations, only, with year nested in location as a main factor and generation and white flowered plant frequency were covariates. To determine if white flower colour frequency was changing through time in hybrid populations, I ran a mixed-model ANCOVA, with year nested in location as a main factor and generation as covariate. Upon finding a significant year nested in location effects, subsequent analyses (described above) were run in Michigan and Ontario locations, separately, followed by post-hoc tests, if required. All mean trait variables were Ztransformed, and further Box-Cox transformed if needed, to meet assumptions of normality. 


\subsubsection{Phenotypic Selection Analysis}

To estimate linear (directional) or quadratic (stabilizing or disruptive) selection on life history traits [days to emergence, days to flower since emergence, and a single size correlate (stem diameter, leaf length, or plant biomass)] for each genotype by population combination within each study, I first standardized reproductive success and life history traits in each study. Reproductive success as an estimate of lifetime fitness (estimated as number of seeds per plant) was standardized for each study (i.e., across all populations in each study) by dividing the individual's reproductive success by the population's mean reproductive success (i.e., relative fitness). To standardize quantitative traits in each study I subtracted the study's mean trait value from the individual trait value and divided by the trait's standard deviation (Lande and Arnold 1983; Zar 1999). To calculate the strength of selection for each population in each study, I performed two multiple regression models (linear and quadratic) that included the standardized life-history traits as predictor variables regressed against relative fitness. From each regression model, I extracted direct selection gradients [linear $(\beta)$ and quadratic $(\gamma)$ ] of emergence and flowering time traits. Additionally, selection differentials for linear and quadratic models were calculated as the covariance of each trait to relative fitness; these measurements estimate the combined direct and indirect selection. All phenotypic selection analyses (PSA) were run in RStudio (using the car package; R Core Team).

Finally, I approached the data from a meta-analytical framework, as well, by extracting weighted linear and quadratic gradients. I weighted emergence and flower time selection gradients by the variance component calculated in the standard PSA, where populations with greater selection confidence (i.e., had less variance) were weighted more than populations with less selection confidence (i.e., greater variance). After weighting the selection values, I re-ran the analyses as described above in section. 4.3.5.2.

\subsubsection{Interpretation of Selection Metrics}

Selection metrics assess the degree to which a trait can affect fitness by regressing the relationship of relative fitness (e.g., number of seeds per plant) to the phenotypic variation of a trait in standard deviation units (Lande and Arnold 1983; Kingsolver et al. 2001). The calculated value is proportional to the strength at which the trait is being selected, such that higher values equate to stronger selection on the trait in the population (i.e., it has a fitness benefit) and vice 
versa for smaller values. Selection on a trait can be either direct or differential. Direct selection, or selection gradients, measure the direct relationship the trait has with number of seeds per plant. Selection differentials, on the other hand, measures the combined direct and indirect relationship a trait has with number of seeds per plant. Selection can affect the distribution of phenotypes in a population, such that: the population experiences directional selection, disruptive selection, or stabilizing selection (Fig. 4.1).

Selection gradients and differentials can be modelled as linear or quadratic, such that: linear selection $(\beta)$ gradients and differentials measure the directional selection on a trait and quadratic $(\gamma)$ selection gradients and differentials measure the curvature, as disruptive or stabilizing, of the selection function (Lande and Arnold 1983; Conner and Hartl 2004; Hartl and Clark 2007; Fig 4.1). Directional selection $(\beta)$ occurs when the environment favours a single mean trait value, causing the current mean trait value to continually shift in a single direction away from the original mean trait value (Fig 4.1). Stabilizing selection $(\gamma)$ occurs when the environment favours a single intermediate trait value and thus disfavours extreme phenotypes of a population (Fig 4.1). Disruptive Selection $(\gamma)$ occurs a change in the environment favours the extreme phenotypic values of the population rather than an intermediate response. Genetic variation for each extreme phenotypic peak is conserved (Fig. 4.1).

Negative linear selection gradients and differentials suggest radish populations are experiencing directional selection for earlier emergence and flowering times, whereas positive values suggest directional selection for later emergence and flower times. Negative values of quadratic selection gradients and differentials suggest populations are experiencing stabilizing selection (i.e., selection towards a single response value), whereas positive quadratic values suggest populations are experiencing divergent selection (i.e., two distinct response values; Kingsolver et al. 2001, Fig.4.1). However, it should be noted that a significant quadratic selection value only implies that selection is non-linear; stabilizing and/or disruptive selection can only be concluded if a significant quadratic selection gradient is coupled with a population graphically exhibiting either type of distribution (Kingsolver et al. 2001; Sherrard et al. 2009).

\subsubsection{Differences in Linear, Quadratic, and Selection Differentials Across Locations}

To test whether crop-traits, overall, were selected against, I ran a non-parametric onesample Wilcoxon signed rank test on the median of selection gradients, weighted gradients, and 
differentials of emergence and flowering time against a hypothesized mean value of zero (IBM SPSS Statistics Version 24, Chicago, IL, USA).

To test for differences in selection gradients, weighted selection gradients, and selection differentials of emergence and flowering times across populations, I ran a nested, mixed-model MANCOVA with genotype, location, their interaction, and year nested in location as the main factors, and generation post-hybridization was included as a covariate. After detecting a significant genotype by year nested in location effect, I ran separate MANCOVA analyses for each location (Michigan and Ontario), followed by one-way ANCOVAs for each response variable, if significant. Finally, I repeated the same model using only hybrid plants, with white flower colour frequency as an additional covariate. In my analyses, I excluded extreme outliers with selection gradients $>10$. When necessary, variables were transformed to conform to assumptions of normality, and analyses were run in R-Studio (Version 1.0.143). 


\subsection{Results}

\subsubsection{Trait Variation in Flower Colour, Emergence and Flowering Time, and Seed Set}

White flowered plants were absent in 45 R. raphanistrum populations and $100 \%$ present in 5 feral $R$. sativus populations, as expected (Section 4.3.1). The 39 hybrid populations varied in their white flowered plant frequency from $0.016 \%$ to $87.4 \%$ (Fig. 4.2d). Wild plants varied in the number of days between planting and seedling emergence from the soil from 2.1 to 59.9 days whereas crop plants emerged between 2.6 to 15.5 days whereas the hybrid populations varied in their emergence schedule from 2.4 to 40.7 days (Fig 4.2a). On average, wild plants flowered earlier (25.9 to 64.4 days after germination) than crop plants (56.6 to 61.4 days after germination). Hybrid populations were intermediate to their parental populations in their flowering schedule (29.1 to 60.25 days after germination; Fig 4.2b). Finally, wild plants produced higher seed sets ( 1 to 8357 seeds per plant) than crop plants (195 to 436 seeds per plant), with hybrid plants producing between 3 and 7427 seeds per plant (Fig 4.3c). Due to a significant year nested in location effect in MANCOVA models, mean emergence time, flowering time, number of seeds per plant and white flower colour frequency in hybrid populations were reported separately for Michigan and Ontario (Emergence: $F_{5,22}=11.28$, $\mathrm{P}<0.0001$; Flowering: $\mathrm{F}_{5,22}-9.20, \mathrm{P}<0.001$; Seed Set: $\mathrm{F}_{5,22}=12.77, \mathrm{P}<0.0001$; White flower colour: $\mathrm{F}_{5,29}=2.85, \mathrm{P}=0.03$ ). Similarly, across populations (wild and hybrid, inclusive) mean emergence time, flowering time, and number of seeds per plant were reported separately for Michigan and Ontario (Emergence: $\mathrm{F}_{6,59}=36.87, \mathrm{P}<0.0001$; Flowering: $\mathrm{F}_{6,59}=16.71, \mathrm{P}<0.001$; Seed Set: $\left.\mathrm{F}_{6,59}=27.37, \mathrm{P}<0.0001\right)$.

Considering only hybrid populations, white flowered plant frequencies in Michigan populations did not vary among years (Table 4.1a, Fig. 4.2d). Michigan hybrid plants emerged 4.5 days earlier in 2004 than 2005 (Table 4.2c, Fig. 4.2a) and flowered $\sim 13$ days earlier in 2005 than 2004 (Table 4.2c, Fig. 4.2b). Finally, hybrid plants produced marginally significantly more seeds in 2005 than 2004 (56\% more seeds; Table 4.2c, Fig. 4.2c). Overall, across Michigan populations, seedling emergence did not vary among genotypes (Table 4.2a) but hybrid plants took nine days longer to flower and were $20 \%$ less fecund than wild populations. Across years (only 2004 and 2005), plants emerged five days earlier and flowered ten days later in 2004 than 
2005 but plants in 2005 were 60\% more fecund than 2004 plants (Figs. 4.2 a, b, and c respectively).

In Ontario, white flower colour frequency was 16\% lower in 2013 than 2012 hybrid populations, with no other pairwise year differences. (Table 4.1d, Fig. 4.2d) Hybrid populations differed between years (Table 4.2d) with respect to emergence time (Fig. 4.2a), flowering time (Fig. 4.2b), and number of seeds per plant (Fig. 4.2c). Hybrid plants grown in 2012 emerged ten and six days later than hybrid plants grown in 2015 and 2016, respectively, and 2015 hybrid plants emerged seven and five days earlier than 2013 and 2016 hybrid plants. Flowering time differed marginally significantly among years, where 2013 hybrid populations flowered six days earlier than 2012 plants, and 2016 plants flowered seven days later than 2015 plants. Finally, number of seeds per plant differed significantly among years (Table 4.3) where hybrid plants grown in 2015 produced approximately three times more seeds than plants grown in 2016 and vastly more seeds produced in 2012 or 2013.

Overall, across all Ontario populations, wild plants emerged two days earlier and flowered five days earlier than hybrid plants (Table 4.2b, Figs. 4.2a and 4.2b). Seedling emergence time and flowering time significantly varied among years. In general, plants grown in 2011 emerged 7 to 19 days earlier than other years (2012 - 2016). In 2012, plants emerged five days and 12 days later than plants in 2013 and 2015, respectively. Finally, plants grown in 2015 emerged seven days later than plants grown 2013 and 2016. Flowering time, however, only differed from plants grown in 2013. Specifically, plants in 2013 flowered 6 days earlier than plants grown in 2012 and 2016. Lastly, the number of seeds per plant significantly differed among years. Plants grown in 2011 produced 9 times more seeds than plants grown in 2012 and 11 times more seeds that plants grown in 2013. Similarly, plants grown in 2016 produced 13 times more seeds than plants grown in 2012 and 17 times more seeds than plants grown in 2013. Finally, 2015 plants were the most fecund across years, producing 3-57 times more seeds than plants from all other years.

\subsubsection{Crop Trait Persistence}

Across all populations and genotypes, directional selection on days to flowering but not emergence deviated significantly from zero in standard models $(\mathrm{P}<0.05)$ and marginally significantly in weighted models $(\mathrm{P}<0.10)$ (Table 4.4a). Populations experienced directional 
selection for earlier flowering (median: $\beta_{\text {standard model }}=-0.08, \beta_{\text {weighted model }}=-4.40 \times 10^{-5}$ ). However, selection differentials across studies showed significant directional and non-linear selection on date of emergence and date of flowering (Table 4.4c). There was weak directional selection for earlier emergence and flowering (median: $\beta_{\text {emergence }}=-0.02, \beta_{\text {flowering }}=-0.05$ ) on plants across studies and non-linear selection on both traits (median: $\gamma_{\text {emergence }}=0.06, \gamma_{\text {flowering }}=0.09$ ). Quadratic selection gradient values did not significantly vary in standard or weighted models for both traits (Table 4.4b). To determine how selection varied across the whole population (wild and hybrid populations combined) and hybrid populations (alone), I ran mixed model MANCOVAs. However, both population-level analyses revealed significant year-nested-in-location effects and results will be reported separately for Michigan and Ontario (Whole population MANCOVA: $\mathrm{F}_{24,216}=1.79, \mathrm{P}<0.05$, weighted: $\mathrm{F}_{24,212}=1.66, \mathrm{P}<0.05$; total: $\mathrm{F}_{28,220}=1.44, \mathrm{P}=0.08$; Hybrid population MANCOVA: standard: $\left.\mathrm{F}_{20,84}=1.69, \mathrm{P}=0.05\right)$. Finally, my hybrid population MANCOVA, revealed that weighted selection gradients and selection differentials did not vary significantly across locations or individual trait ANCOVAs.

\subsubsection{Variation in the Strength of Selection After Hybridization}

In Michigan, linear and quadratic directional selection on emergence and flowering time did not differ between genotypes, among years, or their interaction (Table 4.5a, Figs. 4.3a and b). Total quadratic selection differentials on emergence time showed a marginally significant effect of generation (Table 4.5a), where all plants experienced a shift from stabilizing to disruptive selection as generations $(\mathrm{G})$ increased post-hybridization $\left(\mathrm{G}_{1} \gamma=-0.16, \mathrm{G}_{3} \gamma=0.08, \mathrm{G}_{5} \gamma=0.14\right.$, $\mathrm{G}_{10 \gamma}=0.04$ ). There was a significant genotype-by-generation effect of total quadratic selection differentials on emergence time (Table 4.5a, Fig. 4.4a) where, as generations increased the strength of non-linear selection on hybrid populations oscillated $\left(\mathrm{G}_{1} \gamma=0.20, \mathrm{G}_{3} \gamma=0.03, \mathrm{G}_{5} \gamma\right.$ $=0.09, \mathrm{G}_{10} \gamma=0.03$ ) and selection differentials shifted from strongly negative selection in early generation populations to positive non-linear distribution on later generations of hybrid plants $\left(\mathrm{G}_{1} \gamma=-0.51, \mathrm{G}_{3} \gamma=0.13, \mathrm{G}_{5} \gamma=0.18, \mathrm{G}_{10} \gamma=0.04\right)$. Finally, selection differentials on flowering time had a marginally significant genotype-by-year effect (Table 4.5a, Fig. 4.4b) where, as year progressed, hybrid populations experienced directional selection favouring earlier flowering initially to eventually weak, if any, selection on flowering phenology in later years $\left(\beta_{2004}=-0.17\right.$, $\left.\beta_{2005}=-0.01, \beta_{2010}=0.01\right)$. Due to the lack of clarity when incorporating error values for each 
selection gradient for each study is not presented in this chapter. However, selection differentials for Michigan populations that display error estimates on selection values from each study can be seen in Appendix 7.

In Michigan, selection on emergence varied in marginally significant ways among years on hybrid populations (Table 4.5c) such that 2004 plants experienced directional selection for later emergence and 2005 plants experienced directional selection for earlier emergence $\left(\mathrm{F}_{1,14}=\right.$ $\left.3.96, \mathrm{P}=0.067, \beta_{2004}=0.07, \beta_{2005}=-0.05\right)$. Quadratic selection gradients of days to seedling emergence and flowering differed significantly among years, where Michigan hybrid populations experienced positive, non-linear selection in 2004 but negative, non-linear selection in 2005 (emergence: $F_{1,14}=5.09, P=0.04 ; \gamma_{2004}=0.06$, $\gamma_{2005}=-0.09$; flowering: $F_{1,14}=9.69, P<0.01 ; \gamma_{2004}=$ $\left.0.12, \gamma_{2004}=-0.17\right)$. Other than differences among years, linear and quadratic selection on emergence and flowering times (directional and selection differentials; Table 4.5c) did not vary among generations, years, or their interaction, nor could white flower colour frequencies confidently predict the strength of selection.

In Ontario populations, selection on the phenology of seedling emergence did not vary between genotypes, among years, or their interaction (Table 4.5b, Fig. 4.3a). Selection on flowering time significantly differed between genotypes and among years (Table 4.5b, Figs. 4.3a). Hybrid populations experienced directional selection for early flowering $(\beta=-0.22)$ whereas wild populations experienced directional selection for late flowering $(\beta=0.30)$. In 2012 , 2013, and 2015 plant populations experienced directional selection for early flowering $\left(\beta_{2012}=-\right.$ $\left.0.14, \beta_{2013}=-0.18, \beta_{2015}=-0.16\right)$ whereas 2016 plant populations experienced directional selection for late flowering $\left(\beta_{2016}=0.98\right)$. Relative to 2016 plants, plants in 2011 experienced marginally significantly stronger selection for late flowering $\left(\beta_{2011}=0.08\right)$. Plant populations in $2016\left(\gamma_{2016}=-\right.$ 5.77) experienced marginally significantly stronger stabilizing selection for flowering times compared to 2013 and 2015 populations $\left(\gamma_{2013}=-0.27, \gamma_{2015}=-0.03\right)$. Additionally, 2016 plant populations were marginally significantly different from 2011 and 2012 populations which experienced positive non-linear selection $\left(\gamma_{2016}=-5.77\right.$ versus $\gamma_{2011}=0.13$ and $\left.\gamma_{2012}=0.03\right)$. Selection differentials on emergence time varied between genotypes (Table 4.5b, Fig 4.4a), where hybrid populations experienced five times stronger directional selection for earlier seedling emergence than wild plants $\left(\beta_{\text {hybrid }}=-0.24\right.$ vs. $\left.\beta_{\text {wild }}=-0.05\right)$. Selection differentials on flowering time in quadratic models had a marginally significant genotype effect, where hybrid 
populations experienced almost eight times stronger non-linear selection than wild populations $\left(\gamma_{\text {hybrid }}=0.62\right.$ vs. $\left.\gamma_{\text {wild }}=0.08\right)$. Selection on phenological traits in Ontario hybrid populations (Table $4.5 \mathrm{~d}$ ) did not differ significantly among years.

In Ontario populations, there was a significant genotype by year interaction for selection on emergence (quadratic selection; Table 4.5b) and flowering time (directional selection; Table 4.5b Fig. 4.3b). Emergence times in wild radish populations in 2012 underwent strong non-linear selection $\left(\gamma_{\mathrm{W} 2012}=-1.30\right)$ and differed from 2016 wild populations, which experienced strong disruptive selection $\left(\gamma_{\mathrm{W} 2016}=5.26\right)$. Wild 2016 populations experienced stronger directional selection towards later flowering $\left(\beta_{\mathrm{W} 2016}=2.36\right)$ than 2011 and 2015 wild populations $\left(\beta_{\mathrm{W} 2011}=\right.$ $\left.0.08, \beta_{\mathrm{W} 2015}=0.03\right)$ and differed from 2012 and 2013 wild populations which experienced directional selection for earlier flowering $\left(\beta_{\mathrm{W} 2012}=-0.08, \beta_{\mathrm{W} 2013}=-0.38\right)$. Additionally, wild 2016 populations experienced stronger selection for late flowering than 2013 hybrid populations $\left(\beta_{\mathrm{H} 2013}=0.02\right)$ and differed from 2012, 2015, and 2016 hybrid populations which experienced directional selection for earlier flowering $\left(\beta_{\mathrm{H} 2012}=-0.20, \beta_{\mathrm{H} 2015}=-0.34, \beta_{\mathrm{H} 2016}=-0.41\right)$. Quadratic models modeled similar patterns but with marginally significant differences (Table 4.5b). Specifically, wild 2016 populations underwent stronger negative non-linear selection $\left(\gamma_{\mathrm{w} 2016}=\right.$ 11.39) than $2012\left(\gamma_{\mathrm{W} 2012}=-0.05\right)$ and $2013\left(\gamma_{\mathrm{W} 2013}=-0.66\right)$ populations and differed from 2011 and 2015 wild populations which experienced positive non-linear selection $\left(\gamma_{\mathrm{W} 2011}=0.13, \gamma_{\mathrm{W} 2015}=\right.$ 0.30). Finally, wild 2016 populations experienced marginally significantly stronger negative non-linear selection than 2015 and 2016 hybrid populations $\left(\gamma_{\mathrm{H} 2015}=-0.35, \gamma_{\mathrm{H} 2016}=-0.14\right)$ and differed significantly from 2011 and 2012 populations that experienced positive selection $\left(\gamma_{\mathrm{H} 2011}=0.11, \gamma_{\mathrm{H} 2012}=0.12\right)$.

\subsubsection{Variation in the Strength of Selection using Weighted Linear and Quadratic Models in Crop-wild Hybrid and Wild Populations}

In general, the results of weighted models were similar to the results of non-weighted linear and quadratic directional selection models in both locations (Table $4.6 \mathrm{a}$, c, and d), with the exception of Ontario displaying minor differences in emergence and flowering time selection (Table 4.6b). Thus, I presented only the novel findings from Ontario to minimize repetition.

A significant genotype-by-generation and genotype-by-year effect in weighted models comparing the difference in the strength of selection imposed on Ontario populations suggested 
there are temporal differences in the strength and/or direction of selection acting on crop-derived traits in Ontario (Table 4.6b). Selection on emergence time had a significant generation effect (quadratic: $\mathrm{F}_{1,23}=14.58$, $\mathrm{p}<0.001$ ), where generations experienced non-linear selection on emergence times at varying strengths. Selection on flowering time had significant genotype and year effects in directional models . Hybrid populations experienced significant directional selection for earlier flowering whereas wild populations experienced directional selection for later flowering $\left(\beta_{\text {hybrid }}=-1.00 \times 10^{-4}, \beta_{\text {wild }}=2.00 \times 10^{-4}\right)$; both estimates of the strength of selection were substantially weaker than those generated by non-weighted models. Finally, the strength of selection on flowering time had novel genotype-by-year interactions in quadratic models (Table 4.6b), with few significant pair-wise differences. Wild 2016 populations experienced marginally stronger negative non-linear selection $\left(\gamma_{\mathrm{W} 2016}=-0.51\right)$ for flowering time than 2011 wild populations $\left(\gamma_{\mathrm{W} 2011}=-0.01\right)$; this result stands in direct contrast to the results from the non-weighted models. Unlike non-weighted models, 2016 populations (wild and hybrid, combined) experienced negative, non-linear selection for flowering time $\left(\gamma_{\mathrm{w} 2016}=-0.51\right)$, in contrast to 2013 wild populations which experienced positive, non-linear selection on flowering time $\left(\gamma_{\mathrm{W}_{2013}}=0.07\right)$. Furthermore, the strength of non-linear selection on flowering time of wild 2016 populations $\left(\gamma_{\mathrm{W} 2016}=-0.51\right)$ differed marginally significantly from that hybrid 2012 and 2013 populations, which demonstrated negative non-linear selection $\left(\gamma_{\mathrm{H} 2012}=-6.0 \times 10-4\right.$ and $\left.\gamma_{\mathrm{H} 2013}=-0.005\right)$. Finally, contrary to standard gradient analyses, flowering time of 2016 hybrid populations experienced strong positive non-linear selection $\left(\gamma_{\mathrm{H} 2016}=0.16\right)$, rather than the negative non-linear selection, relative to 2016 wild populations. 


\subsection{Discussion}

By harnessing more than a decade of data measuring crop gene flow into wild radish populations, I have demonstrated that crop-derived traits may be selectively neutral or adaptive and persist in wild radish populations under a diversity of conditions. For instance, hybrid radish populations experienced directional selection on emergence time (in Michigan populations), with no differences in Ontario hybrid populations. Ontario populations, overall (i.e., wild and hybrid), experienced stabilizing selection on earlier flowering time (in Ontario populations, overall). Radish populations, considering Michigan and Ontario together, experienced selection differentials that favoured both crop-derived traits, earlier seedling emergence, and weed-derived traits, early flowering times. This suggests that natural selection can, if sporadically, favour the introgression of crop-derived seedling emergence schedules and foster a diversity of adaptive flowering phenologies across selection environments; considering this, I fail to reject my null and accept my alternative hypothesis. This suggests hybrid populations are selecting for advantageous crop traits (i.e., early seedling emergence), but not flowering time, however this seems to be dependent on the geography of the evolving population. Next, I discuss the influence of selection on crop-trait persistence in weed populations, its effect on hybrid fitness, and the implications of my research on risk assessment of genetically engineered crops.

\subsubsection{Persistence of Crop Traits in Hybrid Populations}

Historically, crop-derived traits have been assumed to be maladaptive and thus are expected to be quickly purged from weedy populations (Gressel 1999; Stewart Jr et al. 2003). For example, traits such as indehiscent fruit (i.e., fruits that do not detach from the stalk), decreased fruit toughness, and decreased seed protection may render weeds susceptible to herbivory (Heredia and Ellstrand 2014). More recently, research has documented the fitness benefits of engineered traits in weed populations (Snow et al. 2003; Ashworth et al. 2016). Pushing the field forward, my research suggests that several non-engineered crop traits are adaptively neutral or beneficial in Raphanus populations, across temporal and spatial scales. For instance, crop-derived white flower colour persisted, sometimes at frequencies as high as $80 \%$, in California, Michigan, and Ontario (Ridley and Ellstrand 2008; Ridley and Ellstrand 2010; Snow et al. 2010; Hovick et al. 2012; Campbell et al. 2016b; Campbell et al. 2016c). Although flower colour appears to be selectively neutral in radish populations (Strauss et al. 2004; Irwin and 
Strauss 2005), white flower colour is genetically correlated with large size (also typically a cropderived trait in hybrid populations) which, in turn, is often correlated with higher seed production in hybrid radish (Campbell et al. 2009b; Weiner et al. 2009; Snow et al. 2010). Maintenance of high frequencies of white-flowered plants or large size in a population may be also mediated by other selective pressures, such as herbivory or pollinator preference (Irwin et al. 2003; Strauss et al. 2004; Irwin and Strauss 2005), and may indirectly favour increased hybrid seed set, as well.

Early seedling emergence is a common crop-derived trait that appears to adaptive in crop-wild hybrid populations (Campbell et al. 2014; Teitel et al. 2016a; Teitel et al. 2016c). Selection weakly favoured early emergence across all studies and strength of selection for early emergence varied among locations. As generations post-hybridization increased, Michigan populations experienced disruptive selection for two distinct seedling emergence phenologies and significant annual variation in directional selection on seedling emergence. Ontario hybrid populations, on the other hand, experienced strong selection differentials favouring early emergence with minimal temporal variation. The strongest selection values occurred in Ontario hybrid populations that evolved under no rain (i.e., drought) and double rain conditions - the two most extreme watering environments (Teitel et al. 2016c; Chapter 2). Similar to Ontario populations, Texas hybrid populations experienced strong selection favouring early emergence and early flowering (Figs. 4.3 and 4.4). However, selection in these populations may have been driven by the competitive environment to which the plants were exposed, such that high competition coupled with low herbicide application levels led to earlier emergence and flowering phenology (Hovick et al. 2012). The selection environment in Texas stands in direct contrast to that experienced by Ohio wild populations, where the strongest selection (which occurred in wild populations exposed to double rain and control rain conditions) favoured early and delayed emergence; a selection environment that favours the versatility of wild seedling emergence phenotypes (Snow and Campbell 2005; Teitel et al. 2016c). Finally, crop populations experienced relatively little selection across Ohio, Michigan, and Ontario, suggesting their phenology was adaptive in these environmental contexts (Snow and Campbell 2005; Nicotra et al. 2010). Crop-wild gene flow may facilitate the transfer of these traits from crop to weedy populations and hybrid populations did not generally experience strong purifying selection against crop-derived phenologies, especially in fluctuating mesic environments (Campbell et al. 2016b; Teitel et al. 2016c). 
Finally, across locations and years, hybrid populations experienced stronger selection for earlier flowering than wild populations, suggesting delayed flowering derived from crop populations is less adaptive than early flowering. Flowering phenology in radish, and other weedy species, is regarded as a key trait that can directly influence lifetime fitness (Conner and Via 1993; Bhatti et al. 2016; Han et al. 2016) because it can lead to a longer flowering season, increased floral abundance, and increased seed production. Earlier flowering in weedy radish populations has been observed across a range of ecological contexts like California, Texas, Australia, and Japan and has been favoured across a range of selection pressures, such as competition, pollinators, temperature, and moisture clines (Hegde et al. 2006; Hovick et al. 2012; Bhatti et al. 2016; Han et al. 2016), demonstrating its significance in weedy radish success.

\subsubsection{Seed Set and Variable Selection Strengths Across Hybrid Populations}

Late-generation hybrid populations produced more seeds per plant compared to early generation hybrid populations in Michigan and Ontario populations. Hybrid pollen infertility, due to chromosomal translocations, is considerably higher in early $\mathrm{F}_{1}$ generation populations (Snow et al. 2001) and declines with generations (Campbell et al. 2006; Snow et al. 2010) - a similar pattern may occur in ovules, but is, as of yet, unmeasured. Overall, hybrid plants were as fecund, or slightly but significantly less fecund, than wild plants in Michigan and Ontario. Early emergence often leads to increased survival in weedy radishes (Campbell et al. 2009b; Teitel et al. 2016a; Teitel et al. 2016c) and early flowering is correlated with increased flower production and larger size (Conner et al. 1996a; Campbell et al. 2009a). Moreover, early flowering time is genetically correlated with increased pollen fertility in hybrid radish (Campbell et al. 2009b). Multiple studies demonstrate that hybrid radish populations have rapidly regained fertility and maintain this fertility in later generations (Chapter 3, Snow et al. 2001, Campbell et al. 2006, Campbell et al. 2009b, Snow et al. 2010). Although, on average, hybrid plants did not outperform wild progenitors, hybrid plants were more successful than wild progenitors in California and Texas (Campbell et al. 2006; Hovick et al. 2012), particularly in California (Campbell et al. 2006; Hegde et al. 2006).

Finally, selection acting on hybrid populations appeared to be more variable than that acting on wild populations. The reasons behind this variability may be three-fold. First, hybrid populations were still segregating, therefore, more variation in the phenotype among hybrid 
populations than wild populations may exist while hybrid populations were evolving towards a steady phenotypic state (Arnold and Hodges 1995). Second, these crop-wild hybrid populations represented populations from a range of generations, $\left(F_{1}\right.$ to $\left.F_{10}\right)$, also likely within a single population given that radish has an extensive seed bank; earlier segregating generations may experience stronger selection than late generation hybrids (Kingsolver et al. 2001; Campbell and Snow 2007; Campbell et al. 2009a). Finally, strength of selection for crop trait phenologies may depend of the frequency of crop traits within the population (Lande and Arnold 1983) - a value, in my analysis, quantified only for flower colour across studies. Variation is a key characteristic of hybrid populations (Campbell et al. 2009a; Goulet et al. 2017) and although early generation hybrids may lead to less predictable results, the outcome, overall, remains consistent in that crop traits can persist in hybrid populations.

\subsubsection{Moving Forward with Risk Assessment of Crop Gene Flow}

Many studies have assessed the risk that genetically engineered traits may be transferred to weed populations (Stewart Jr et al. 2003; Warwick and Stewart 2005). Pollen-mediated transfer of glyphosate resistance from cultivated creeping bentgrass (Agrostis stolonifera L.) into wild populations has been documented (Reichman et al. 2006) and can travel as far as $21 \mathrm{~km}$ (Watrud et al. 2004) - traits like this may have concerning consequences on the subsequent control of weed populations. Similarly, cultivated varieties of Brassica rapa and Helianthus annuиs have transferred insect resistance (i.e., Bt traits) into weedy hybrid populations, resulting in crop-wild hybrid populations with increased fitness compared to wild progenitors in particular environments (Snow et al. 2003; Vacher et al. 2004; Whitney et al. 2006; Liu et al. 2015a). However, genetically engineered traits represent only a small fraction of the traits transferred to weed populations and many traits associated with domesticated plants could also influence weed population success. Here, I studied the consequences of the introgression of two phenological traits into weed populations but these are only a fraction of the traits that wild populations can gain from crop systems. Considering the long-term persistence (Snow et al. 2010) and environmental resiliency of crop-traits, mitigation or risk-assessment policy evaluating the risk of crop gene into wild or weedy relatives must be explored for multiple traits across a range of environments. 
Radish is an excellent model organism for studying the rate at which crop genes introgress into wild populations (Klinger et al. 1991) due to their insect-pollinated, outcross mating strategy which is shared with many crops (e.g., canola, cabbage, cucumber, carrots, and certain grains like rye and buckwheat). Crop-derived, neutral molecular markers have persisted for a decade in wild radish populations in one location at a relatively high rate ( 10 to 35\%, Snow et al. 2010). Here, I demonstrated that crop alleles will persist across a wide range of environments, as well. Consistent with previous research in the field (Bartsch et al. 1999; Snow et al. 2010; Campbell et al. 2016a) this confirms that crop gene introgression into wild populations may have persistent consequences for the genetic diversity of non-crop populations across a range of environments.

Finally, results of my work suggest that variation in gene flow and selection can favour a diversity of adaptive phenologies, including domesticated traits, in weedy radish populations across environments. Crop gene introgression into wild populations - whether selectively disadvantageous, neutral, or positive - may have implications on conservation and economic efforts. Selectively disadvantageous crop-to-wild gene flow can affect genetic diversity in wild populations through the loss of rare alleles and/or lineages (Wolf and Wade 2009; Campbell et al. 2016a). Successful introgression can be selectively neutral, producing no phenotypic change, but could increase the genetic diversity in introgressed populations, relative to wild populations (Kost et al. 2015), replace wild alleles with crop alleles (not changing allelic diversity but rather allelic composition) or reducing genetic diversity within unmanaged populations to make weed populations more genetically homogenous (Campbell et al. 2016a; Todesco et al. 2016). Initially, this may not impact wild populations; however, environmental perturbations to a genetically depauperate population may limit its ability to demographically recover or adaptively evolve (Ladizinsky 1985; Slatkin 1987; Ellstrand et al. 1999). Finally, adaptive, crop-derived traits can sweep through populations, reduce genetic diversity in recipient populations and foster the evolution of more successful hybrid weeds (Ridley and Alexander 2016; Todesco et al. 2016); as demonstrated by California wild radish ( $R$. raphanistrum $\times$ R. sativus; Campbell et. al 2009a) (Campbell et al. 2009). From an economic perspective, characterizing selective forces across landscapes that promote crop-wild hybridization and foster hybrid populations can allow researchers to predict and implement pre-emptive strategies to mitigate gene-flow and prevent weed outbreak; this can occur at large-geographical scales. For example, if early emergence (a 
crop trait) is identified as selectively advantageous in response to drought and/or excess rain fall (Chapter 2- section 2.4.3), then agricultural advice can be provided to farmers to promote early tillage to control hybrid weed populations. Our understanding of the variable nature of crop gene flow and selection, therefore, may aid in creating predictive strategies in the broad-scale management and monitoring of weed species. 


\subsection{Table List}

Table 4.1: ANOVA model testing the white flower colour frequency across years in a) Michigan and b) Ontario populations. a) In Michigan an ANCOVA model was run testing changes white flower colour frequency across two years $(2004,2005)$ and four generations (Generations: 1, 3, $5,10)$, with generation acting as a covariate. b) In Ontario an ANOVA model was run testing changes in white flower colour frequency across four years $(2013,2014,2015,2016)$. Generation was inherently correlated with year between 2013 and 2014 but 2015 and 2015 populations represented a single generation (generation 5). Due to this confounding variable, generation could not be run as a covariate in the Ontario model. F-statistics are presented; to indicate significant differences: $\mathrm{ns}, \mathrm{P}>0.10 ;+, \mathrm{P}<0.10 ; *, \mathrm{P}<0.05 ; * *, \mathrm{P}<0.01 ; * * *, \mathrm{P}<$ 0.001 .

\begin{tabular}{lcc}
\hline Location \& Parameters & df (num., den.) & F-statistic \\
\hline a) Michigan & & \\
\hline Year & 1,18 & 0.91 \\
Generation & 1,18 & $8.51^{* *}$ \\
\hline b) Ontario & & \\
\hline Year & 3,11 & $3.60^{*}$ \\
\hline
\end{tabular}


Table 4.2: ANCOVA model mean trait responses of days to emergence, days to flower, and number of seeds per plant (seed set) in a) Michigan wild and hybrid populations, b) Ontario wild and hybrid populations, c) Michigan hybrid populations, and d) Ontario hybrid populations.

Numerator and denominator degrees of freedom are represented in subscripts for each parameter. Analyses were run in R-Studio Version 1.0.143. F-statistics are presented to indicate significant differences: $\mathrm{ns}, \mathrm{P}>0.10 ;+, \mathrm{P}<0.10 ; *, \mathrm{P}<0.05 ; * *, \mathrm{P}<0.01 ; * * *, \mathrm{P}<0.001$.

\begin{tabular}{|c|c|c|c|}
\hline \multirow{2}{*}{ Location \& Parameters - num. df, den. df } & \multicolumn{3}{|c|}{ Response Variables (F-statistic) } \\
\hline & Days to Emergence & Days to Flower & Seed Set \\
\hline \multicolumn{4}{|l|}{ a) Michigan } \\
\hline Genotype $_{1,34}(\mathrm{G})$ & $0.33^{\mathrm{ns}}$ & $23.24^{* *}$ & $1.51^{\mathrm{ns}}$ \\
\hline $\operatorname{Year}_{1,34}(Y)$ & $14.65^{* *}$ & $31.87^{* *}$ & $5.80^{*}$ \\
\hline Generations $_{1,34}$ (Gen) & $0.45^{\mathrm{ns}}$ & $0.86^{\mathrm{ns}}$ & $0.06^{\mathrm{ns}}$ \\
\hline $\mathrm{G} \times \mathrm{Y}_{1,34}$ & $0.22^{\mathrm{ns}}$ & $1.15^{\mathrm{ns}}$ & $0.00^{\mathrm{ns}}$ \\
\hline $\mathrm{G} \times \mathrm{Gen}_{1,34}$ & $0.00^{\mathrm{ns}}$ & $2.70^{\mathrm{ns}}$ & $0.04^{\mathrm{ns}}$ \\
\hline \multicolumn{4}{|l|}{ b) Ontario } \\
\hline $\mathrm{G}_{1,25}$ & $7.28^{*}$ & $33.78^{* *}$ & $0.90^{\mathrm{ns}}$ \\
\hline $\mathrm{Y}_{4,25}$ & $43.42^{* *}$ & $5.67^{* *}$ & $59.28^{* *}$ \\
\hline $\mathrm{G} \times \mathrm{Y}_{3,25}$ & $1.01^{\mathrm{ns}}$ & $0.75^{\mathrm{ns}}$ & $1.01^{\mathrm{ns}}$ \\
\hline \multicolumn{4}{|l|}{ c) Michigan Hybrid Populations } \\
\hline$Y_{1,15}$ & $3.27^{+}$ & $24.47^{* *}$ & $3.58^{+}$ \\
\hline White (W) 1,15 & $0.00^{\mathrm{ns}}$ & $6.21^{*}$ & $0.20^{\mathrm{ns}}$ \\
\hline $\operatorname{Gen}_{1,15}$ & $0.51^{\mathrm{ns}}$ & $0.34^{\mathrm{ns}}$ & $0.06^{\mathrm{ns}}$ \\
\hline $\mathrm{Y} \times \mathrm{W}_{1,15}$ & $0.02^{\mathrm{ns}}$ & $2.33^{\mathrm{ns}}$ & $1.41^{\mathrm{ns}}$ \\
\hline $\mathrm{W} \times \mathrm{Gen}_{1,15}$ & $3.20^{+}$ & $5.61^{*}$ & $8.10^{*}$ \\
\hline \multicolumn{4}{|l|}{ d) Ontario Hybrid Populations } \\
\hline $\mathrm{Y}_{3,7}$ & $37.74^{* *}$ & $5.17^{*}$ & $38.08^{* *}$ \\
\hline $\mathrm{W}_{1,7}$ & $0.11^{\mathrm{ns}}$ & $0.00^{\mathrm{ns}}$ & $0.38^{\mathrm{ns}}$ \\
\hline $\mathrm{Y} \times \mathrm{W}_{3,7}$ & $3.52^{+}$ & $1.73^{\mathrm{ns}}$ & $0.54^{\mathrm{ns}}$ \\
\hline
\end{tabular}


Table 4.3: The mean number of seeds per plant in Ontario hybrid populations across years. Mean \pm SE (groups marked with different letters represent significantly different groups).

\begin{tabular}{cc}
\hline Year & Number of Seeds per Plant \\
\hline 2012 & $155.17 \pm 56.73^{\mathrm{a}}$ \\
2013 & $64.84 \pm 23.10^{\mathrm{a}}$ \\
2015 & $6141.13 \pm 509.27^{\mathrm{b}}$ \\
2016 & $1313.81 \pm 483.83^{\mathrm{c}}$ \\
\hline
\end{tabular}

Table 4.4: Wilcoxon signed-rank test comparing (a) directional selection gradients, (b) quadratic selection gradients, and (c) selection differentials between wild and crop-wild hybrid populations. Directional selection gradients (a) represent the direct linear relationship a trait has on the number of seeds per plant (i.e., fitness) whereas quadratic selection gradients (b) represent the non-linear relationship a trait has on fitness. Selection differentials (c) represent the total direct and indirect direction and quadratic selection, separately, occurring for the trait. In both (a) and (b) parameters, I used standard and weighted models for both traits. Standard models that evaluate the raw-value of the trait extracted from the phenotypic selection analysis (PSA). Weighted models used selection values weighted by variance component calculated in the standard PSA; populations with greater selection confidence (i.e., had less variance) are weighted more than populations with less selection confidence (i.e., greater variance). For a detailed description, see methods section 4.3.4.2. Analysis was run in IBM SPSS Statistics 24. Sample size $(\mathrm{N})$ and Wilcoxon signed-rank test statistics are presented; to indicate significant differences: $\mathrm{ns}, \mathrm{P}>0.10 ;+, \mathrm{P}<0.10 ; *, \mathrm{P}<0.05 ; * *, \mathrm{P}<0.01 ; * * *, \mathrm{P}<0.001$.

\begin{tabular}{llc}
\hline Gradient Parameters & N & Standardized Test Statistic \\
\hline a) Directional Selection Gradient & & \\
\hline Emergence Time & 71 & $1.13^{\mathrm{ns}}$ \\
Flower Time & 73 & $1.93^{*}$ \\
Weighted Emergence Time & 71 & $1.1^{\mathrm{ns}}$ \\
Weighted Flower Time & 73 & $1.9^{+}$ \\
\hline b) Quadratic Selection Gradient & & \\
\hline Emergence Time & 71 & $-0.65^{\mathrm{ns}}$ \\
Flower Time & 73 & $-0.37^{\mathrm{ns}}$ \\
Weighted Emergence Time & 71 & $-0.65^{\mathrm{ns}}$ \\
Weighted Flower Time & 73 & $0.18^{\mathrm{ns}}$ \\
\hline c) Selection Differential & & \\
\hline Emergence Time (Directional) & 71 & $1.99^{*}$ \\
Flower Time (Directional) & 73 & $3.57^{* *}$ \\
Emergence Time (Quadratic) & 71 & $-2.85^{* *}$ \\
Flower Time (Quadratic) & 73 & $-3.93^{* *}$ \\
\hline
\end{tabular}




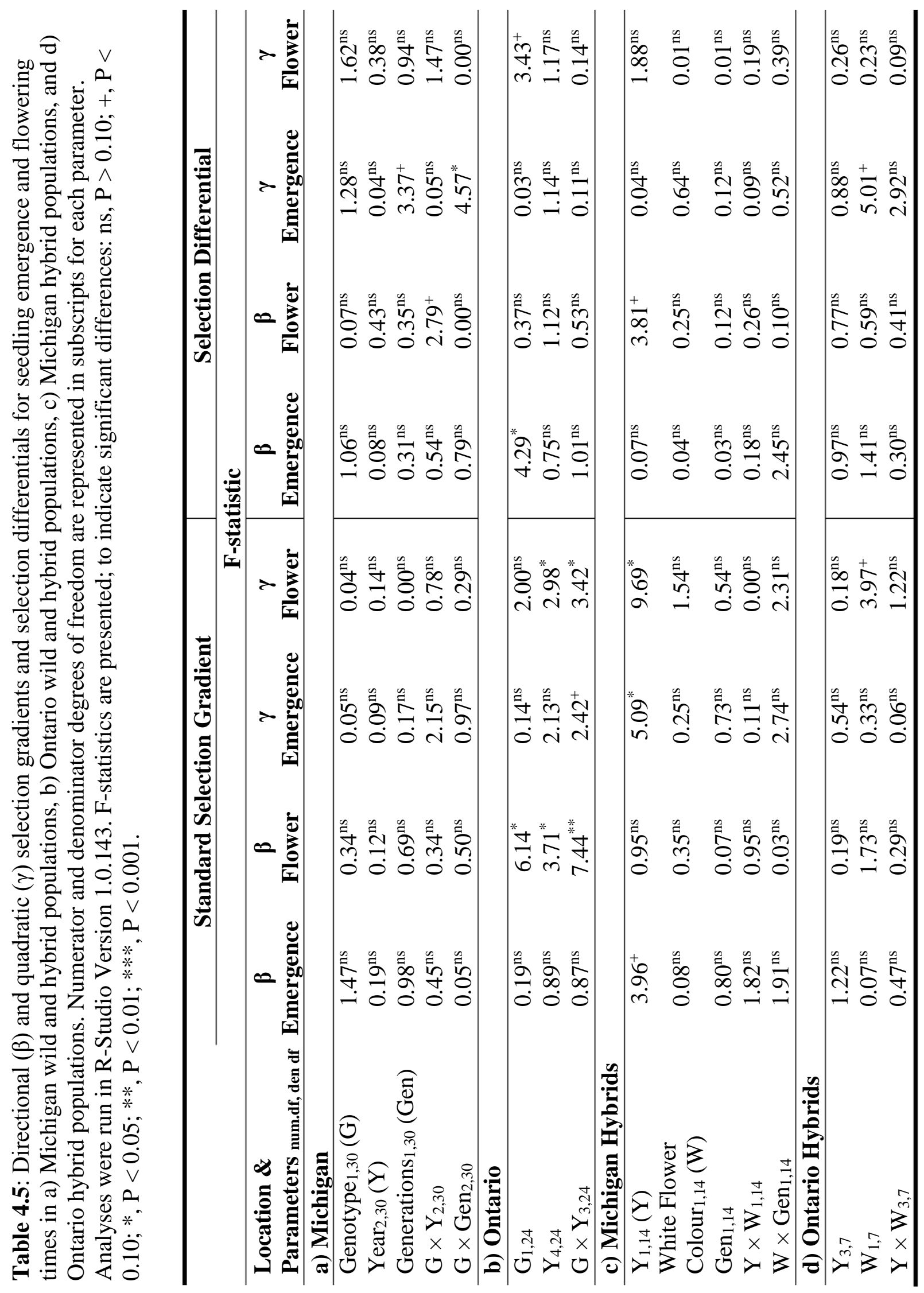


Table 4.6: Directional ( $\beta)$ and quadratic $(\gamma)$ weighted selection gradients in a) Michigan wild and hybrid populations, b) Ontario wild and hybrid populations, c) Michigan hybrid populations, and d) Ontario hybrid populations. Numerator and denominator degrees of freedom are represented in subscripts for each parameter. Analyses were run in R-Studio Version 1.0.143. F-statistics are presented; to indicate significant differences: $\mathrm{ns}, \mathrm{P}>0.10 ;+, \mathrm{P}<0.10$; ${ }^{*}, \mathrm{P}<0.05 ;{ }^{* *}, \mathrm{P}<0.01$; ***, $\mathrm{P}<0.001$.

\begin{tabular}{|c|c|c|c|c|}
\hline \multirow{3}{*}{ Location \& Parameters num.df, den df } & \multicolumn{4}{|c|}{ Weighted Selection Gradients } \\
\hline & \multicolumn{4}{|c|}{ F-statistic } \\
\hline & $\beta$ Emergence & $\beta$ Flower & $\gamma$ Emergence & $\gamma$ Flower \\
\hline \multicolumn{5}{|l|}{ a) Michigan } \\
\hline Genotype $_{1,30}(\mathrm{G})$ & $1.43^{\mathrm{ns}}$ & $0.21^{\mathrm{ns}}$ & $0.10^{\mathrm{ns}}$ & $1.29^{\mathrm{ns}}$ \\
\hline $\operatorname{Year}_{2,30}(\mathrm{Y})$ & $0.15^{\mathrm{ns}}$ & $0.12^{\mathrm{ns}}$ & $0.10^{\mathrm{ns}}$ & $0.14^{\mathrm{ns}}$ \\
\hline Generations $_{1,30}(\mathrm{Gen})$ & $1.09^{\mathrm{ns}}$ & $0.62^{\mathrm{ns}}$ & $0.19^{\mathrm{ns}}$ & $0.07^{\mathrm{ns}}$ \\
\hline $\mathrm{G} \times \mathrm{Y}_{2,30}$ & $0.75^{\mathrm{ns}}$ & $0.31^{\mathrm{ns}}$ & $1.71^{\mathrm{ns}}$ & $0.46^{\mathrm{ns}}$ \\
\hline $\mathrm{G} \times \mathrm{Gen}_{2,30}$ & $0.04^{\mathrm{ns}}$ & $0.43^{\mathrm{ns}}$ & $0.24^{\mathrm{ns}}$ & $0.00^{\mathrm{ns}}$ \\
\hline \multicolumn{5}{|l|}{ b) Ontario } \\
\hline $\mathrm{G}_{1,23}$ & $0.23^{\mathrm{ns}}$ & $5.67^{*}$ & $0.38^{\mathrm{ns}}$ & $2.80^{\mathrm{ns}}$ \\
\hline $\mathrm{Y}_{4,23}$ & $0.72^{\mathrm{ns}}$ & $3.86^{*}$ & $1.07^{\mathrm{ns}}$ & $0.70^{\mathrm{ns}}$ \\
\hline $\operatorname{Gen}_{1,23}$ & $0.41^{\mathrm{ns}}$ & $0.09^{\mathrm{ns}}$ & $14.58^{* *}$ & $0.07^{\mathrm{ns}}$ \\
\hline $\mathrm{G} \times \mathrm{Y}_{3,23}$ & $0.34^{\mathrm{ns}}$ & $7.28^{* *}$ & $1.91^{\mathrm{ns}}$ & $5.40^{* *}$ \\
\hline \multicolumn{5}{|l|}{ c) Michigan Hybrids } \\
\hline $\mathrm{Y}_{1,14}(\mathrm{Y})$ & $4.80^{*}$ & $0.76^{\mathrm{ns}}$ & $6.24^{*}$ & $0.83^{\mathrm{ns}}$ \\
\hline White Flower Colour $1,14(\mathrm{~W})$ & $0.10^{\mathrm{ns}}$ & $0.43^{\mathrm{ns}}$ & $0.81^{\mathrm{ns}}$ & $2.38^{\mathrm{ns}}$ \\
\hline $\mathrm{Gen}_{1,14}$ & $0.85^{\mathrm{ns}}$ & $0.10^{\mathrm{ns}}$ & $0.08^{\mathrm{ns}}$ & $0.25^{\mathrm{ns}}$ \\
\hline $\mathrm{Y} \times \mathrm{W}_{1,14}$ & $2.29^{\text {ns }}$ & $0.89^{\text {ns }}$ & $0.41^{\mathrm{ns}}$ & $0.22^{\mathrm{ns}}$ \\
\hline $\mathrm{W} \times \mathrm{Gen}_{1,14}$ & $2.46^{\mathrm{ns}}$ & $0.04^{\mathrm{ns}}$ & $4.25^{+}$ & $7.40^{*}$ \\
\hline \multicolumn{5}{|l|}{ d) Ontario Hybrids } \\
\hline $\mathrm{Y}_{3,7}$ & $0.70^{\mathrm{ns}}$ & $0.18^{\mathrm{ns}}$ & $1.05^{\mathrm{ns}}$ & $0.96^{\mathrm{ns}}$ \\
\hline $\mathrm{W}_{1,7}$ & $0.32^{\mathrm{ns}}$ & $1.79^{\mathrm{ns}}$ & $0.05^{\mathrm{ns}}$ & $2.41^{\mathrm{ns}}$ \\
\hline $\mathrm{Y} \times \mathrm{W}_{3,7}$ & $0.74^{\mathrm{ns}}$ & $0.27^{\mathrm{ns}}$ & $0.05^{\mathrm{ns}}$ & $0.19^{\mathrm{ns}}$ \\
\hline
\end{tabular}




\subsection{Figure List}

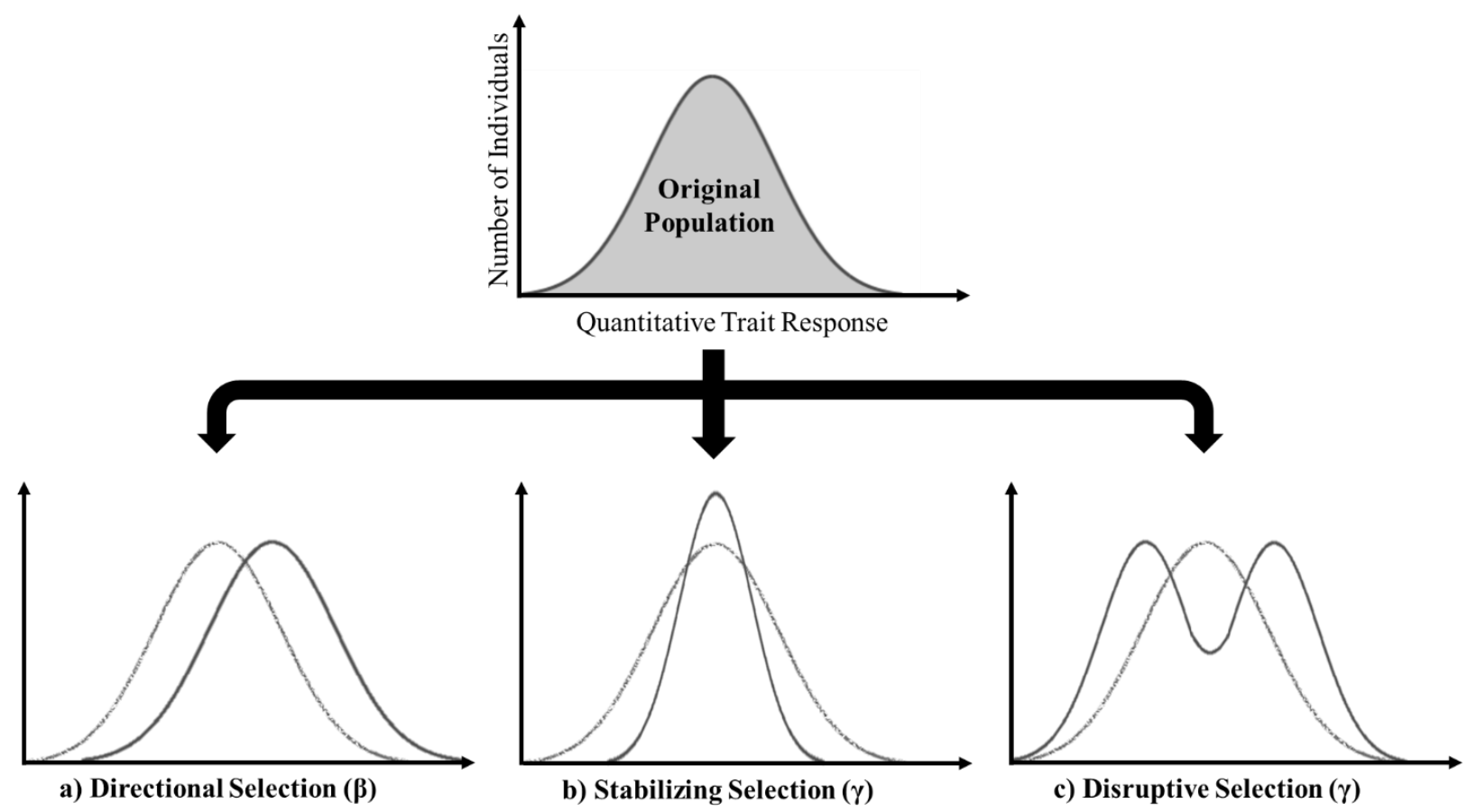

Figure 4.1: Selection on a trait can be expressed as either directional or non-linear selection. Selection gradients measure the direct effect the trait has on fitness. Selection can affect the distribution of phenotypes in one of three ways: a) directional, b) non-linear, stabilizing, or c) non-linear, disruptive selection. Directional selection $(\beta)$ is the change in the environment favours a single mean trait value, but maintain genetic variation, causing the allele frequency to continually shift in a single direction away from the original mean trait value. Stabilizing selection $(\gamma)$ : a change in the environment decreases the genetic diversity of a population and stabilizes selection on a single particular trait value. Disruptive Selection $(\gamma)$ : a change in the environment favours the extreme phenotypic values of the population rather than an intermediate response. Genetic variation for each extreme phenotypic peak is conserved. 

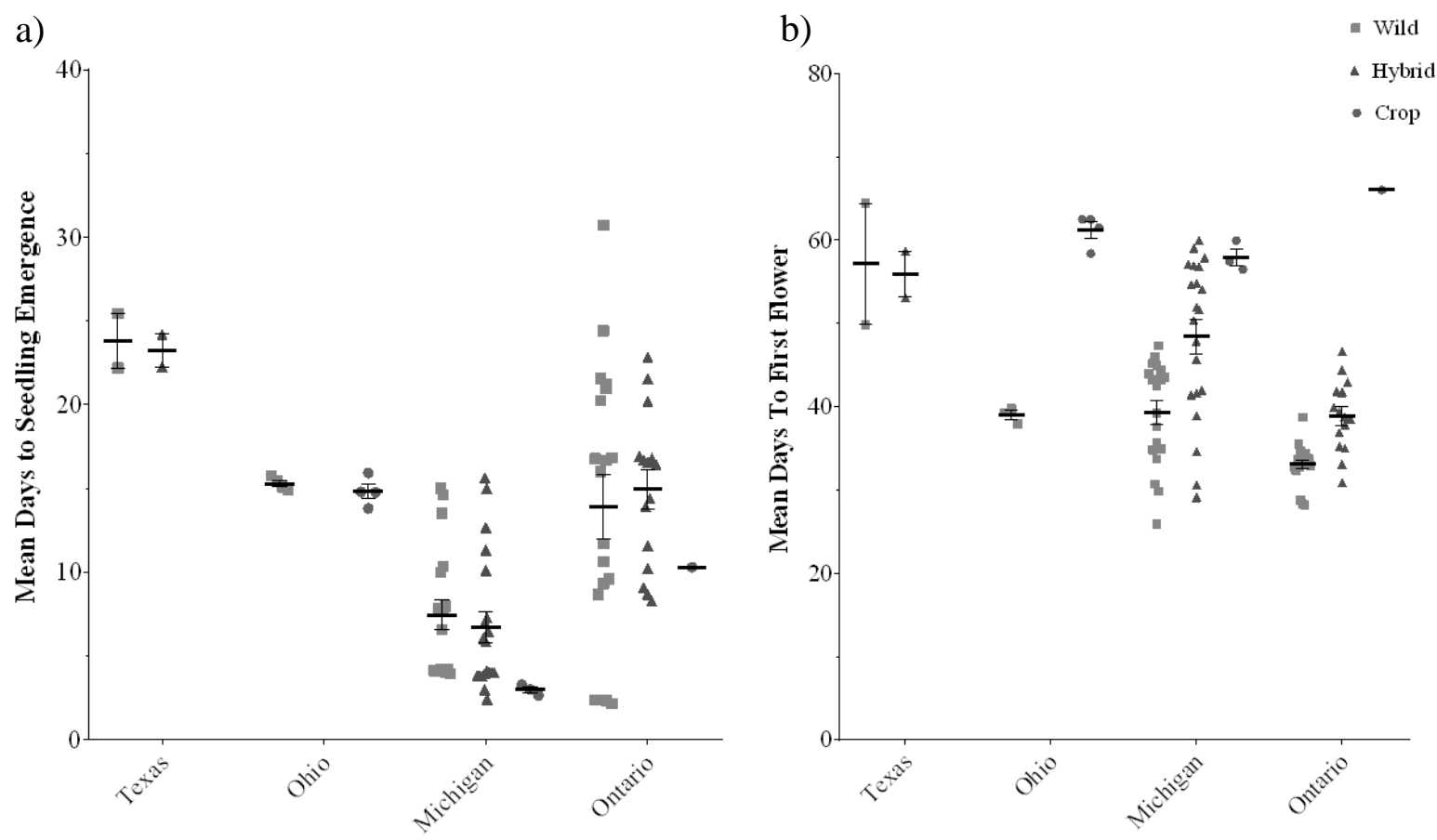

c)

d)
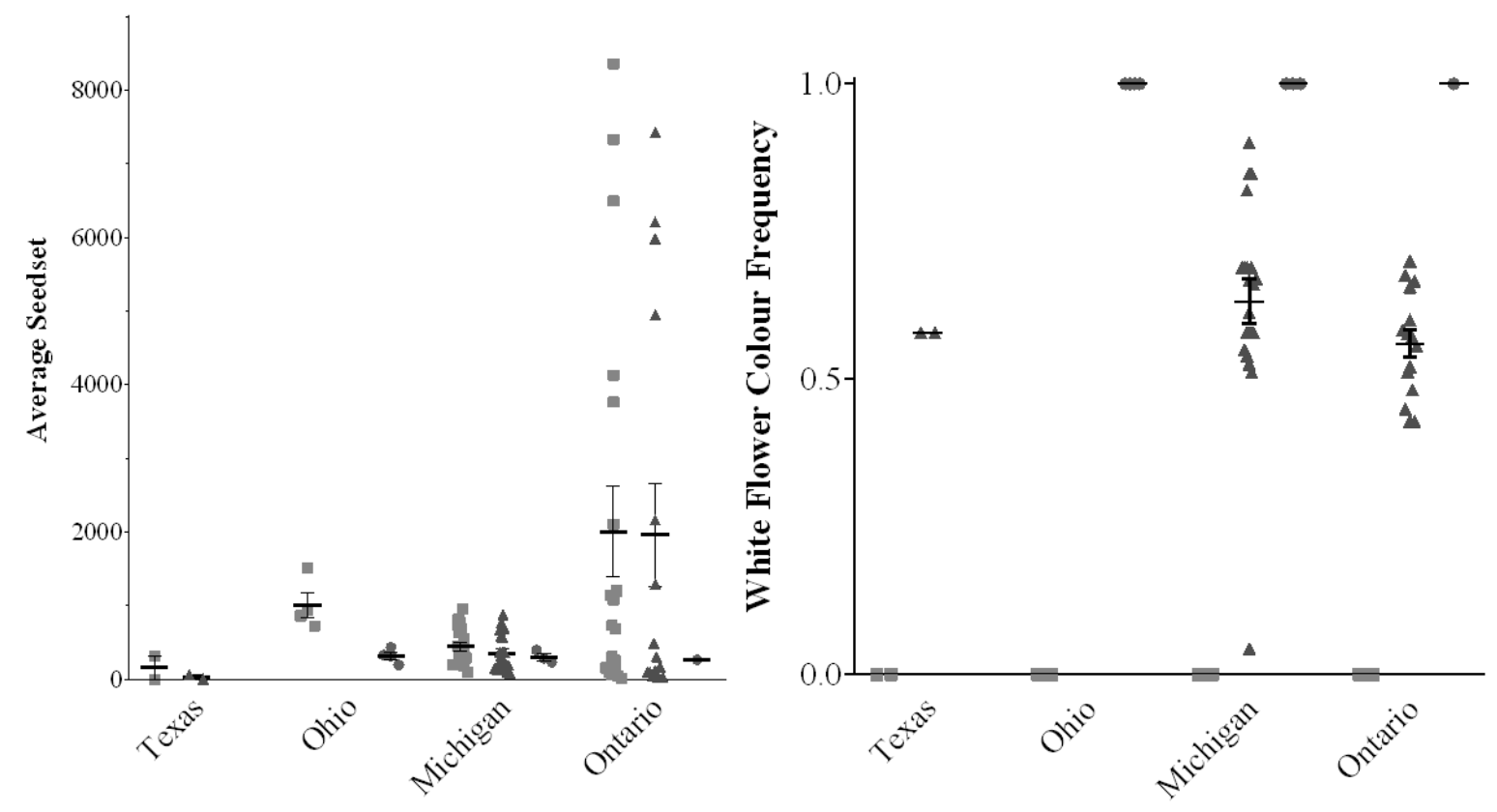

\section{Location}

Figure 4.2: Data points represent the mean days to (a) seedling, (b) first flower, and (c) number of seeds per plant, and (d) white flower colour frequency of a population $\mathrm{x}$ genotype combination of wild radish (Raphanus raphanistrum, orange square), hybrid radish (R. raphanistrum $\times R$. sativus, teal triangle), or crop radish (Raphanus sativus, grey circle) grown in one of four locations: Houston, TX, Columbus, OH, Pellston, MI; King City, ON). The black bars represent the mean of all the populations $( \pm \mathrm{SE})$ in each location; because of the volume of data, some error estimates are very small. 


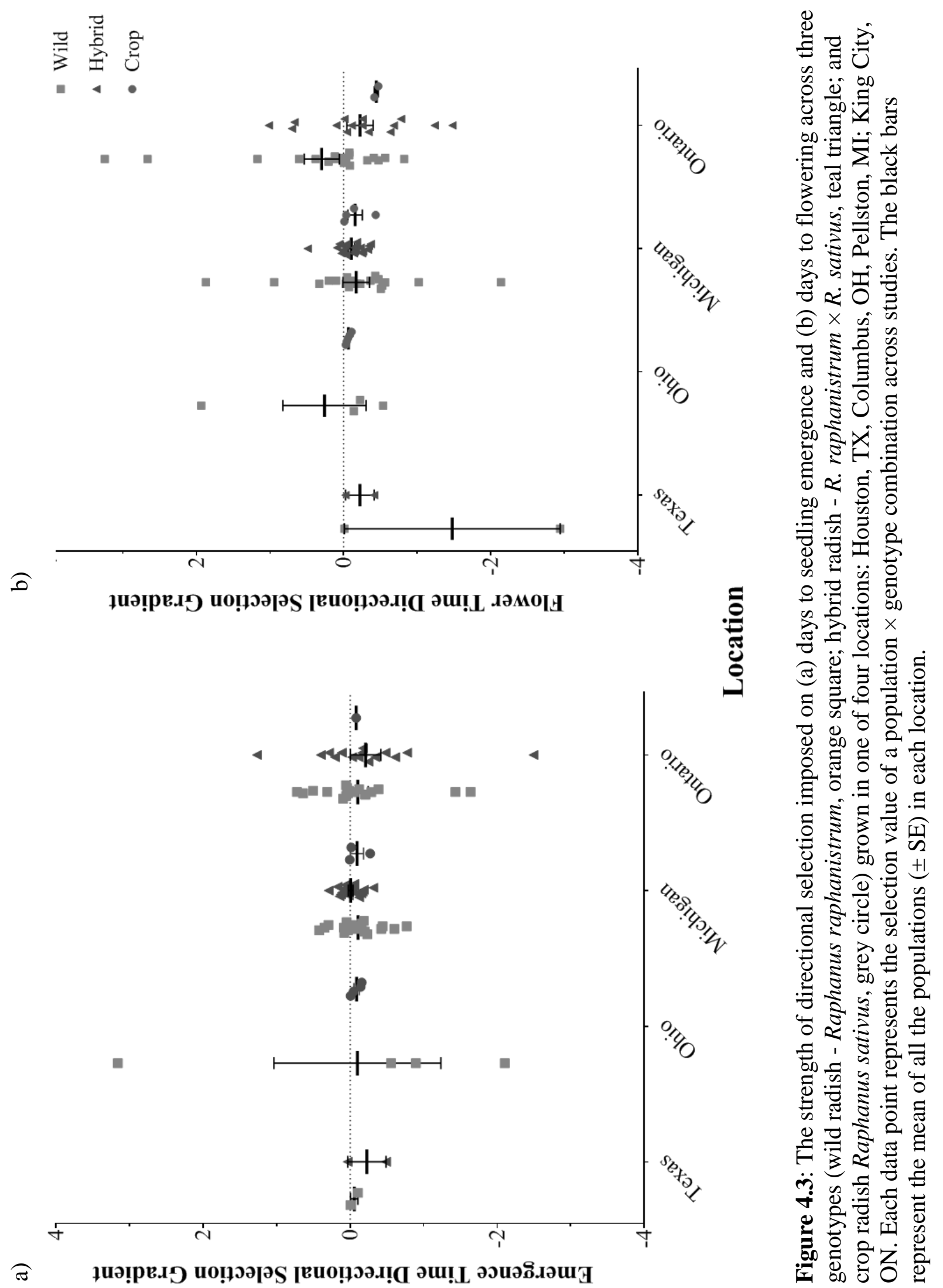



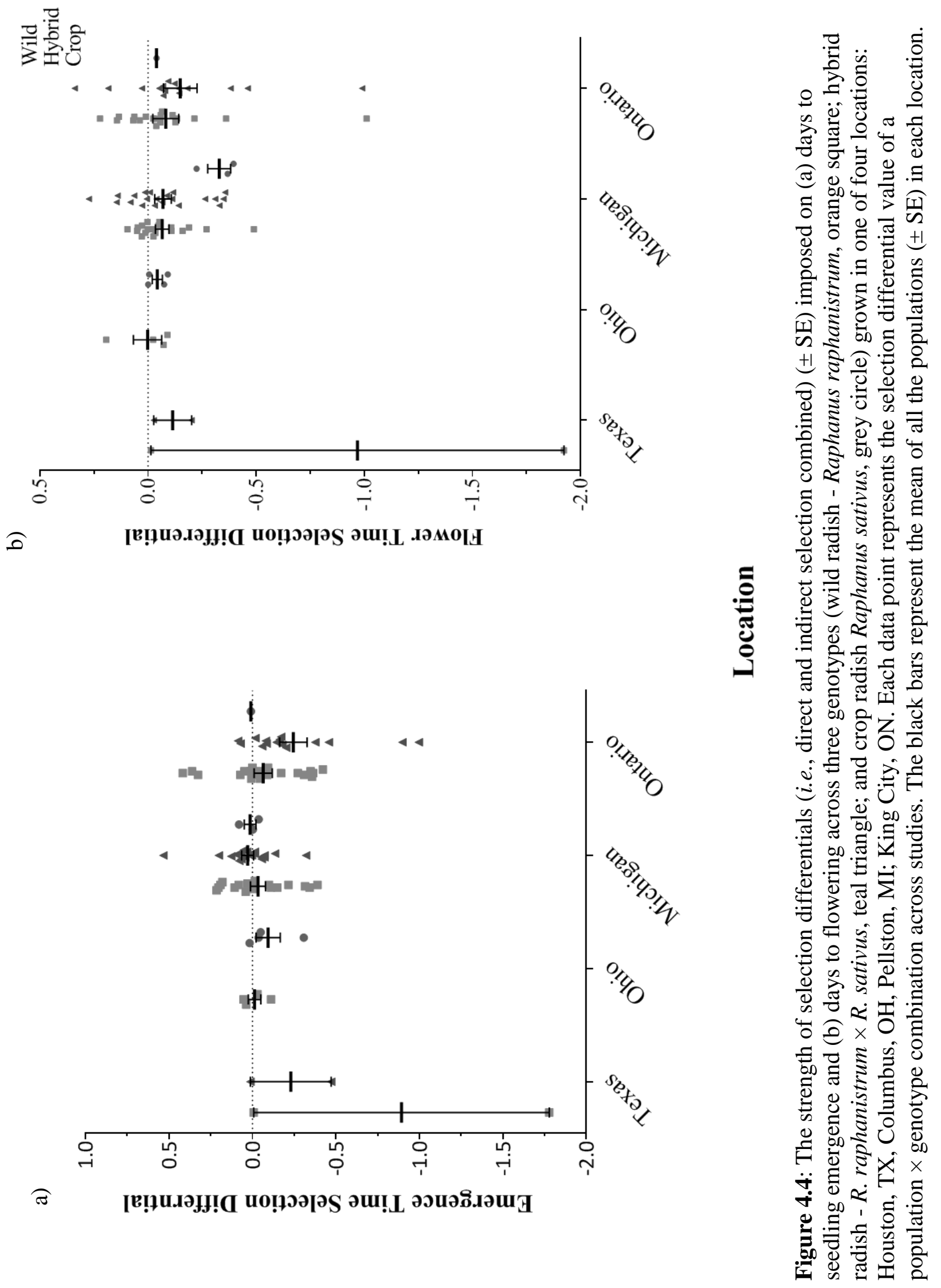


\section{Chapter 5: Synthesis and the Future Study of Crop-Wild Hybridization}

\subsection{Synthesis}

Previous research has demonstrated that crop-wild hybridization can lead to dramatic improvements in relative fitness in radish (Campbell et al. 2006; Campbell and Snow 2007; Ridley and Ellstrand 2008; Hovick et al. 2012). Further, crop alleles that contribute adaptive traits to weed populations may persist for long periods of time in weedy populations (Snow et al. 2010). Furthermore, evolution in crop-wild hybrid complexes has been known to proceed much more quickly than in non-hybrid populations (Wu and Campbell 2006; Campbell and Snow 2009; Campbell et al. 2009b; Ellstrand et al. 2010; Campbell et al. 2016c). Broadly, rates of introgression may be partly determined by ecological context (i.e., shared pollinators, shared habitats, etc.; Stanton 1987, Brunet and Sweet 2006, Arnold and Martin 2010, Van Etten et al. 2013; Fig 1.1 in Chapter 1). My research has contributed to this field of knowledge by testing how environmental context would influence introgression. With that in mind, I measured the consequences of environmental variation, especially moisture availability, for a) on life-history and fitness traits and strength of natural selection on domesticated traits in weedy, advancedgeneration, crop-wild, Raphanus populations $\left(\mathrm{G}_{5}\right)$ relative to their wild progenitors - and $\mathrm{b}$ ) our ability to measure rates of contemporary evolution.

Since 2010, four moisture treatments that range from relatively dry to excessively wet have been imposed on 20 wild and 20 crop-wild hybrid experimental populations. In Chapter 2, I discovered that plant biomass, in general, could effectively predict the number of flowers, fruits, and seeds produced by plants; however, above-ground biomass as a correlate of success did not vary between genotypes (wild, hybrid), selection history (NR - no rain, CU - control unsheltered, CS - control sheltered, DR - double rain), or their interaction. My results reinforce that biomass can be an accurate predictor of plant reproductive success (i.e., number of flowers, number of fruits, and total seeds per plants), with the strength of this relationship increasing among watering environments with weaker correlations between genotypes. After five generations of selection, I found that hybridization affected life-history traits seedling emergence time and enhanced PS II functioning (but only in DR environments) relative to wild radish plants and the lifetime fecundity trait of biomass. Interestingly, watering history (i.e., evolving in no rain, control unsheltered, or double-rain environments) did not alter life-history traits. However, when 
grown in current double rain environments, evolving in double rain conditions pre-disposed plants to greater success (i.e., greater biomass) relative to invading control-sheltered plants, irrespective of their genotype (wild or hybrid).

The environmental context under which plants are measured can profoundly alter conclusions that adaptive evolution has occurred (Clausen et al. 1948). Current rate of evolution metrics do not account for heritable or non-heritable phenotypic plasticity but rather treat the change in mean phenotype as synonymous with environmentally insensitive genetic variation expressed in the phenotype. However, phenotypic plasticity as an adaptive response is a welldocumented, common, and adaptive strategy for many plants (e.g., Sultan 1995; Williams et al. 1995; Richards et al. 2006; Ghalambor et al. 2007; Moczek et al. 2011). Overlooking the influence of environmental variation on phenotype can lead to erroneous estimates of rates of evolution. Although others have recognized this caveat in estimating rates of evolution, my work is the first to explicitly demonstrate the erroneous conclusions that can be made. Based on data collected from two common gardens of $\mathrm{G}_{5}$ plants, estimates of the magnitude and direction of contemporary evolution due to annual variation in environmental context differed significantly, particularly for wild populations (Chapter 3). In fact, evolutionary rate estimates were more inconsistent in magnitude, and at times contradictory, between years for phenological traits. In contrast, hybrid populations exhibited more consistent phenotypes between years. In response to these erroneous estimates, designing common garden field experiments that compare differences in genetic variance between divergent populations (i.e., calculating $\mathrm{V}_{\mathrm{E}}$ and removing its confounding effect) can improve the accuracy of evolutionary rate estimates. When careful experimental design is not an option, including the use of environmentally canalized traits, genetic variance heritability estimates, or molecular markers, when available, may decrease the inaccuracy of evolutionary rate estimates.

After more than a decade of studies measuring gene flow from crop populations into wild radish populations, I have presented overwhelming evidence that crop phenology was selectively advantageous in weedy populations in some ecological contexts, even several generations posthybridization, and thus crop alleles will likely persist in wild radish populations in, at least, four places in North America (Chapter 4). Radish populations experienced strong divergent selection on flowering time and, overall, experienced total directional selection towards earlier emergence and flowering times. Although the strength of selection on these phenological traits spatially 
varied, hybrid radish remained as fecund as wild plants across experiments and over generations. Moreover, this work has demonstrated that introgression can occur successfully across a range of environments and generate competitive weeds. Chapters 2-4, collectively, have established the need to further study the environmental context and varying selection pressures that promote the success of crop-wild hybrids to understand how it may influence the success of crop traits in weed populations.

\subsection{Experimental Limitations}

In retrospect, I would like to suggest a few experimental design improvements that could strengthen my conclusions. In Chapter two, space was limited in my experimental design and thus limited my sample size. Along with more replicates of hybrid and wild plants, acquiring crop plants in 2015 may have allowed for better comparison of hybrid relative to both progenitors (crop and wild radish). Greater shelter area would have allowed better spacing between plants and reduced above-ground competition between radish plants. Increasing spacing between shelters, as well, may have allowed for better rain capture due to fewer impeding structures; but I believe this is of less concern considering water capture methods were fairly efficient based on previous experimental work (Campbell et al. 2016b; Teitel et al. 2016c). Similar to radish studies in California or Texas (see Table 1.1), measuring over a longer growth season - from May to August, for example - may capture phenological, morphological, and/or physiological traits representative of progenitors (i.e., earlier emergence from crops) that, then, may drive differences between hybrid and wild radish. Additionally, I found that biomass could be an effective indictor of plants success (total seed production). However, the sample size of my allometric experiment used to determine this relationship may have been too small and not provide enough statistical power to detect differences between genotypes and/or watering histories. Therefore, future work would benefit from collecting data on flower and fruits numbers, and total seed production from a larger sample size to develop stronger allometric relationships. Finally, collecting data associated with other selection pressures, such as herbivory or competition, in addition to soil moisture may have driven and elucidated differences in fecundity between wild and hybrid plants.

My conclusions in Chapter 3 were limited due to low replication among families. Planting greater family replicates would have allowed me to separate genetic $\left(V_{G}\right)$ and 
environmental variance (VE) phenotypic values (see Dlugosch and Parker 2008). From this, I would have been able to calculate the independent effect genetic variance and environmental variance have on evolutionary rate estimates. Having these values would allow for an appropriate comparison to determine the degree to which $\mathrm{V}_{\mathrm{E}}$ confounds evolutionary rate estimates. Additionally, due to time constraints, I could not calculate evolutionary rate estimates for fecundity, or a correlate such as biomass, which may have been better trait candidates - due to their high heritability (Weiner et al. 2009; Younginger et al. 2017) - and provided better evolutionary rate estimates.

Finally, in approaching Chapter 4, the major limitation occurred within the replicate number of populations grown in Ohio and Texas. This data would have provided an interesting comparison with respect to the contrasting climates, particularly Texas, of Ontario and Michigan. Having California fecundity data would have also provided interesting conclusions on the strength of trait selection in hybrid populations, considering the most successful invasion of hybrid radish has occurred in this area. Finally, if I was to physically collect more data from previous studies, gather information on additional selection pressures - for example, temperature, soil moisture, pollinator presence, and/or herbivory - would allow one to eliminate multiple confounding environmental factors and determine which pressure(s) promote relative hybrid success.

\subsection{Recommended Future Work}

A natural progression of this work would be to evaluate multiple selection pressures abiotic and biotic - in addition to the moisture profiles to determine what affects the strength of trait selection. Temperature can affect several underlying population characteristics such as floral display time, size, colour, sex ratio of populations, and the ratio of open to closed flowers (Barrett and Harder 1996; Barrett 2014). In populations of fireweed (Chamerion angustifolium) and a New Zealand tree (Pseudowintera colorata) the duration of floral display shortened through the growing season (Wells and Lloyd 1991; Sargent and Roitberg 2000). Changes in the duration, or time of floral display can create periods of phenological mismatch, where flowering does not overlap with conspecifics or occur when pollinators are present; this can decrease the probability of successful ovule fertilization (Forrest et al. 2010). Following this, floral display may affect successful fertilization opportunities and can affect the occurrence and persistence of 
crop-to-wild gene flow, as well (Irwin and Strauss 2005). Crop-wild hybridization occurs globally, so determining the ecological contexts (e.g., soil moisture, soil quality, herbivores, pollinators, and/or inter-specific competition) that influences crop-wild hybrid success in a diversity of ecological contexts may identify selection pressures that promote the evolution of crop-wild hybrid weeds. For example, if we observe hybrid populations from two continents having substantially different environments but experience selection for similar traits and reproductive output - it would be interesting to compare and contrast the underlying genetic structure and environments in both populations that promote, and maintain, hybrid weed populations (Kinnison and Hairston 2007; Ellstrand and Rieseberg 2016) . Identifying shared selection pressures that promote and maintain hybridization between geographies can allow researchers to predict and implement pre-emptive strategies to mitigate gene-flow and prevent weed outbreak; this can occur at large-geographical scales. Alternatively, if geographic pressures do not overlap, research on crop trait escape must be implemented locally and policy created at regional, rather than global, scales.

Biotic factors and their interaction with abiotic factors can be important in facilitating crop-trait transfer into wild populations. In $R$. raphanistrum radish populations, for example, Pirimova et al. (2015) found that floral traits - corolla diameter and anther lengths - increased in size in moist conditions. Morphological changes in floral traits, induced by interacting abiotic shifts in the environment, can directly facilitate pollinator movement and fertilization success and may vary when comparing weed success. Additionally, crop-wild hybrid radish have the ability to respond more resiliently in response to conspecific competition in Michigan and Texas (Campbell and Snow 2007; Hovick et al. 2012). Other variants of crop-wild hybrids, Helianthus species for example, have shown increased success in response to herbivore pressures (Whitney et al. 2006). Belowground biotic interactions may exist, as well. Fungal endophytes, for example, are microbial fungal species that live symbiotically within some plant species. The presence of fungal endophytes have been known to alter the microbial community within the rhizosphere (i.e., soil community directly influenced by the root system of plants). Changes in these microbial communities have influenced aboveground plant productivity (Clay 2001; Clay and Schardl 2002; Lemons et al. 2005). Similarly, new cultivars of crop tomato inoculated with an endophyte strain in their roots demonstrated an increase in the production of plant growth hormones (PHP) and increased lateral root hair growth (Abbamondi et al. 2016). Although crop- 
wild hybrid radish are not typically endophyte infected (Ocampo 1980), the rhizosphere of hybrid plants may be altered that promote their success relative to wild progenitors (e.g., Saxena et al. 2002; Picard and Bosco 2005). Future work, therefore, may consider measuring the success of hybrid radish through an integrative multi-trophic approach in natural environments, where along with a single environmental pressure (e.g., soil moisture) researchers incorporate a biotic component (e.g., pollinators, herbivores, or microbial community composition). A multi-trophic approach may clarify the underlying mechanisms that promote, or not, crop-trait introgression into wild populations, and the selection pressures that enhance trait persistence.

\subsection{Implications}

In Chapter 2, I ran a common garden to evaluate the evolutionary and ecological consequences of the single largest experimental evolution experiment (Maron et al. 2004; Thorpe et al. 2005; Whitney et al. 2006; Sharpe et al. 2008; Vitasse et al. 2009; Keller and Taylor 2010; Eizaguirre et al. 2012; Bunbury-Blanchette et al. 2015) using organisms that aren't single celled or digital (Lenski et al. 1991; Elena and Lenski 2003; Barrick et al. 2009). With this work, I have evaluated and challenged the current approach by agencies evaluating the risks of transgenes whereby crop-wild hybridization consequences are assumed to be consistent across major ecological gradients, like water clines - a rather significant assumption. To my knowledge, I am running the first assessment of advanced-generation, crop-wild hybrids that considers abiotic environmental variability in the assessment of relative fitness. My results reinforce that biomass can be an accurate predictor of plant reproductive success (i.e., number of flowers, number of fruits, and total seeds per plants), with the strength of this relationship increasing among watering environments with weaker correlations between genotypes. Hybrid plants, in general, were larger across environments irrespective of their role as an invader or resident species. Watering history, on the other hand, did not provide a distinct advantage to plants relative to invading plants, unless they were plants from double rain environments. This suggests that hybridization may be a stronger determinant than watering history and better determinate of invasive success. Risk assessments evaluating the invasive success of weed radish populations, therefore, need to focus on hybridization and other, or a combination of, environmental clines as selective pressures. 
Chapter 3 was the first study of any organism to explicitly measure the magnitude of variation in evolutionary rates across environments and provide insights into the influence of environmental variation on the measurement of broad-scale evolutionary patterns. Although others have acknowledged the role of environmental variation influencing phenotypic variation and making it difficult to estimate evolutionary rates accurately (Hendry and Kinnison 1999; Bone and Farres 2001; Gorné and Díaz 2017), researchers have never explicitly established the extent to which environmental variation interferes with an accurate estimate of contemporary rate of evolution metrics and have continued to publish evolutionary rates in the absence of this information. My research demonstrated that failing to account for environmental variation on the phenotype in estimates of evolutionary rates can lead to radically divergent estimates, and thus erroneous interpretations of evolutionary patterns in biological populations. Furthermore, my research demonstrated evolutionary trajectories differed, sometimes radically, despite experiencing similar natural selection environments, especially when considering wild populations. My research has established a new standard in the design and execution of rate of evolution studies that requires either a change to the calculation of metrics or experimental design.

Finally, my work from Chapter 4 assessed the potential for variation in rates of crop trait introgression after crop-wild hybridization. Results of my work suggest that variation in gene flow and selection can favour a diversity of adaptive phenologies including domesticated traits in weedy radish populations across environments. Additionally, crop gene introgression into wild populations - whether selectively disadvantageous, neutral, or positive - may have implications on the genetic diversity, composition, and persistence of weedy populations across environments. Crop-to-wild gene flow can affect genetic diversity in wild populations through outbreeding depression via demographic swamping (loss of rare alleles or lineages) or genetic swamping (loss of a progenitor species but not necessarily the alleles) (Wolf and Wade 2009; Campbell et al. 2016a). Crop traits can be selectively neutral, producing no phenotypic change, and have several outcomes in wild populations: successful introgression which could increase genetic diversity in wild populations that do not possess the crop-derived trait (Kost et al. 2015) or changes in the frequency of an allele that is present in both crop and wild populations such that wild populations are more genetically homogenous (i.e., decreased genetic diversity) (Campbell et al. 2016a; Todesco et al. 2016). This, initially, may not impact wild populations; however, a 
detrimental perturbation (e.g., caused by environmental changes) may eradicate a population and, due to limited or negligible genetic diversity, prevent population recovery/evolution (i.e., genetic drift) (Ladizinsky 1985; Slatkin 1987; Ellstrand et al. 1999). Finally, selectively advantageous crop-derived traits can sweep through populations, reducing genetic diversity in recipient populations and potentially creating more successful hybrid plants that are capable of outcompeting wild progenitors (Ridley and Alexander 2016; Todesco et al. 2016); as demonstrated by California wild radish ( . raphanistrum $\times R$. sativus). The variable nature of gene flow and selection, therefore, may create localized hot- and low-spots of introgression and impede broad-scale management and monitoring strategies of crop gene flow.

\subsection{Contribution in Creating Effective Policy}

Weed management policy implement practices that both mitigate and minimize damage from current weeds and manage the escape of crop traits that may enhance the success of weeds. Weed management policy is created using an integrative approach by including farmers or seed manufacturers, stakeholders (e.g., herbicide/pesticide companies), provincial experts, and government and academic researchers to develop new policy (AAFC 2016) that identifies gaps in management strategies, with three goals: 1) establish knowledge through problem-specific data collection; 2) develop weed control solutions based on the knowledge generated in the first goal; and 3) communicate strategic solutions to stakeholders (AAFC 2016). The work from my dissertation contribute to steps one and two of the policy-creating process.

The work from this dissertation can aid as a stepping stone in creating effective policy and mitigation strategies in assessing the invasion risk associated with weeds generated by cropto-wild gene flow. At face value, results from Chapter 2 (soil moisture and hybrid success) suggest watering environment, exclusively, does not seem to increase the risk of creating invasive weed species. However, results from Chapter 4 (geography and selection on crop traits) demonstrate that depending on the environment, crop traits can persist in weedy populations and create weeds that are as aggressive as progenitor populations. This suggests that abiotic variation, due to a wide range of environmental factors, can affect weediness. Although preliminary work measuring a single abiotic factor (soil moisture) did not enhance hybrid weed success, several abiotic factors - individually and/or interactively - may enhance success. 
Therefore, multiple abiotic (and potentially biotic) factors should be considered before making predictions about crop traits' persistence in weedy populations.

This work is far from complete before concrete policy can be implemented, however, it does enact the precautionary principle in environmental decision making where precautionary measures should be taken, even if results are not fully established or significant (Kriebel et al. 2001, AAFC 2016). Applicable precautionary measures within my work include: 1) preemptively taking actions to prevent crop-to-wild gene flow creating invasive weeds and 2) exploring a range of factors that may promote gene flow between crop and wild plants (Kriebel et al. 2001). Prior to introducing plants to new areas, research addressing the influence of the environment on the spread of crop-wild hybrid weeds is an important step in taking preventative measures. My work is some of the first to evaluate the effect of soil moisture on crop-wild hybridization and, although results suggest no difference in success, it raises new questions on what facilitates the increased success of crop-wild hybrid weeds. Although, research evaluating environmental variation on invasive potential can take time to properly test and evaluate, thoroughly-researched work (through all aspects) can be critical in creating and implementing effective policy. 


\section{Appendix 1 - Frequency and Volume of Water Applied to Experimental Plots}

During my 2015 and 2016 common garden experimental years, natural rainfall varied over the growing season between common garden years, with a cumulative rainfall of approximately $307.8 \mathrm{~mm}$ in 2015 and $206.7 \mathrm{~mm}$ in 2016 (nearest weather station: Buttonville, Ontario 4351'39" N, 79²2'07" W; Government of Canada 2018). Prior to a rainfall event in both years, I added approximately $100 \mathrm{~mL}$ of water to initiate seedling emergence. In 2015 common garden plots, there were 12 applications of water (i.e., rainfall events) from June to August: nine in June, two in July, once in August. Similarly, in 2016 common garden plots, there were nine applications of water from June to August: four in June and July, and once in August. *Quantified estimates of rainfall are based on data from Buttonville, Ontario as data associated with King City, Ontario was not available.

General Values and Calculations:

2015 cumulative rainfall (June - August): $307.8 \mathrm{~mm} \rightarrow 0.3078 \mathrm{~m}$

2015 cumulative rainfall (June - August): $206.7 \mathrm{~mm} \rightarrow 0.2067 \mathrm{~m}$

Volume added in June to initiate seedling emergence prior to a rain event: $0.100 \mathrm{~L}$

Shelter Area: $2.44 \mathrm{~m}$ by $3.05 \mathrm{~m}=7.44 \mathrm{~m}^{2}$

Conversion to Volume in Litres: $1 \mathrm{~m}^{3}=1000 \mathrm{~L}$

\section{$\underline{2015 \text { Experiment }}$}

$7.44 \mathrm{~m}^{2} \times 0.3078 \mathrm{~m}=2.29065 \mathrm{~m}^{3} \times 1000 \mathrm{~L}=2290.65 \mathrm{~L}$ of cumulative volume of water applied per plot over three-month experiment

$2290 \mathrm{~L} \div 12$ rain events $=190.89 \mathrm{~L}$ per barrel $+0.100 \mathrm{~L}$ (initial amount added prior to emergence)

No rain $(N R)$ plots received:

$(190.89 \mathrm{~L} \times 0)+0.100 \mathrm{~L}$ (frequency of rain events in June $)=0.100 \mathrm{~L}$

$(190.89 \mathrm{~L} \mathrm{x} 0)$ (frequency of rain events in July) $=0 \mathrm{~L}$

$(190.89 \mathrm{~L} \times 0)$ (frequency of rain events in August $)=0 \mathrm{~L}$

Control shelter (CS) plots received:

$(190.89 \mathrm{~L} \mathrm{x} \mathrm{9)}+0.100 \mathrm{~L}$ (frequency of rain events in June $)=1718.11 \mathrm{~L}$ in June

$(190.89 \mathrm{~L} \times 2)($ frequency of rain events in July) $=381.78 \mathrm{~L}$ in July

$(190.89 \mathrm{~L} \mathrm{x} \mathrm{1})($ frequency of rain events in August $)=190.09 \mathrm{~L}$ August

Double rain (DR) plots received two applications of water (one collected form DR and one from $N R$ ). Therefore, the base value DR plots received was 381.78 L (190.89 $x 2$ applications).

$(381.78 \mathrm{~L} \mathrm{x} 9)+0.100 \mathrm{~L}$ (frequency of rain events in June) $=3436.12 \mathrm{~L}$ in June

$(381.78 \mathrm{~L} x$ 2) (frequency of rain events in July) $=763.56 \mathrm{~L}$ in July

$(381.78 \mathrm{~L} \mathrm{x} 1)($ frequency of rain events in August $)=381.78 \mathrm{~L}$ August 


\section{$\underline{2016 \text { Experiment }}$}

$7.44 \mathrm{~m}^{2} \times 0.2067 \mathrm{~m}=0.2067 \mathrm{~m}^{3} \times 1000 \mathrm{~L}=1537.84 \mathrm{~L}$ cumulative volume of water applied per plot across the three months

$1537.84 \mathrm{~L} \div 9$ (the frequency of water events) $=170.87 \mathrm{~L}$ per barrel $+0.100 \mathrm{~L}$ (initial amount added prior to emergence)

No rain $(N R)$ plots received:

$(170.87 \mathrm{~L} \times 0)+0.100 \mathrm{~L}$ (frequency of rain events in June $)=0.100 \mathrm{~L}$

$(170.87 \mathrm{~L} x$ 0) (frequency of rain events in July) $=0 \mathrm{~L}$

$(170.87 \mathrm{~L} x$ 0) (frequency of rain events in August) $=0 \mathrm{~L}$

Control shelter $(C S)$ plots received:

$(170.87 \mathrm{~L} \mathrm{x} \mathrm{4)}+0.100 \mathrm{~L}$ (frequency of rain events in June $)=683.58 \mathrm{~L}$ in June

$(170.87 \mathrm{~L} \mathrm{x} 4)$ (frequency of rain events in July) $=683.48 \mathrm{~L}$ in July

$(170.87 \mathrm{~L} x$ 1) (frequency of rain events in August) $=170.87$ L August

Double rain $(D R)$ plots received two applications of water (one collected form DR and one from NR). Therefore, the base value DR plots received was 341.74 L (170.87 22 applications).

$(341.74 \mathrm{~L} \mathrm{x} 4)+0.100 \mathrm{~L}$ (frequency of rain events in June $)=1367.06 \mathrm{~L}$ in June

(341.74 L x 4) (frequency of rain events in July) $=1366.96 \mathrm{~L}$ in July

(341.74 L x 1) (frequency of rain events in August) $=341.74$ L August 


$$
\begin{gathered}
\text { Appendix } 2 \text { - Traits Calculated in darwins }(\mathbf{d}) \\
\operatorname{darwin}(d)=\frac{\left[\left(\ln \bar{x}_{2}\right)-\left(\ln \bar{x}_{1}\right)\right]}{\Delta t} \quad(\text { Equation } A 2-1),
\end{gathered}
$$

where, the mean trait values of control shelter wild and hybrid (CS) populations were represented by $\bar{x}_{1}$ and trait values of wet (DR), dry (NR), or control unsheltered (CU) evolved wild and hybrid populations were represented by $\bar{x}_{2}$ in Equation A2 - 1 (Table A1.1). The $\Delta t$ is the change through time in millions of years $\left(\Delta t=\frac{5}{1000000}=5.0 \times 10^{-6}\right)$ (Haldane 1949; Gingerich 1993, 2001). Finally, I calculated the rate of evolution on molecularly evaluated trait in darwins on data collected from Snow et al. (2010) (Table A1.2). 


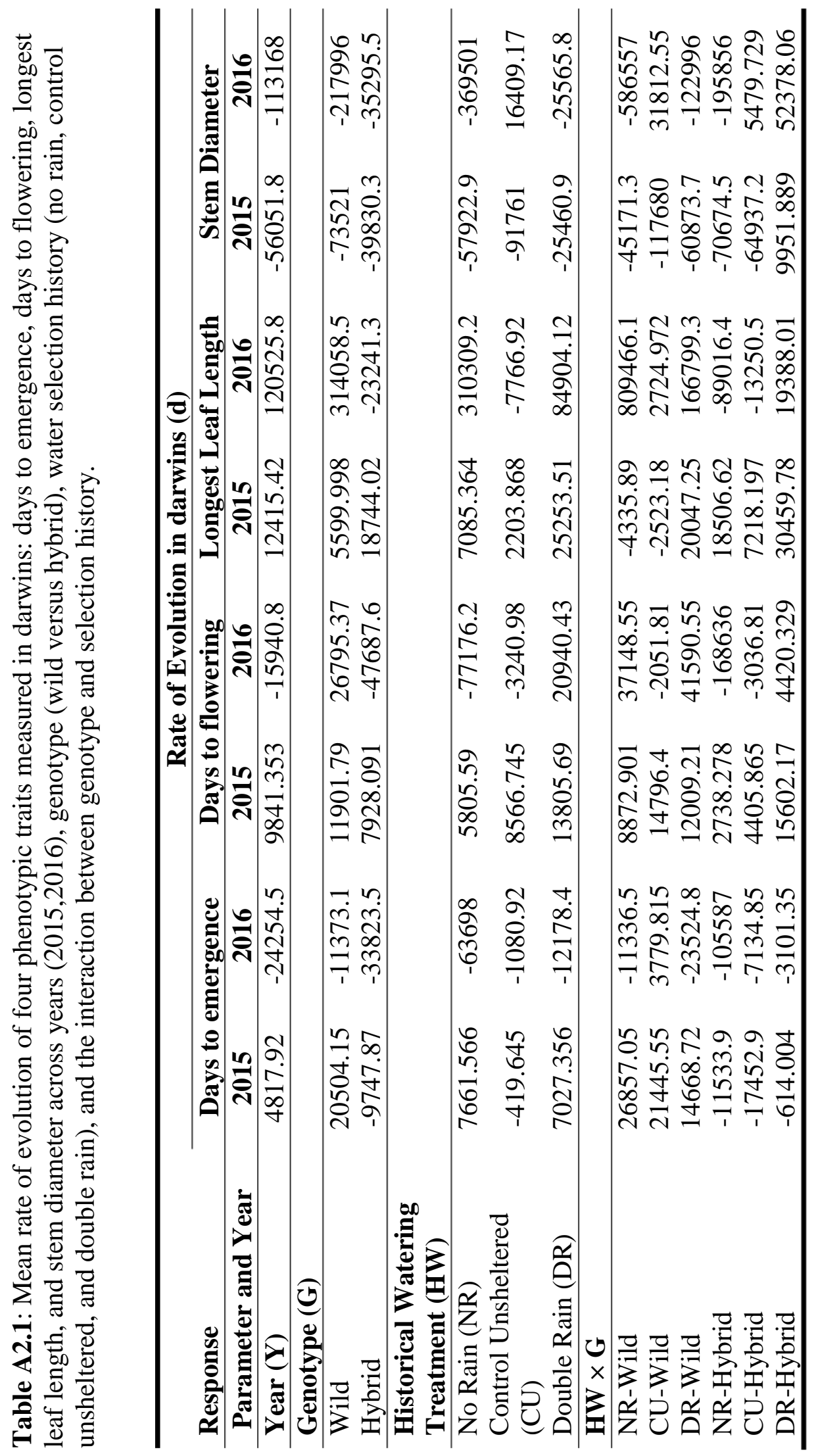

(continued) 
Table A2.2: The calculated the rate of evolution of three crop-specific molecular markers [glucose-6-phosphate isomerase enzyme (GPI), phosphoglucomutase enzyme (PGM), and white flower colour frequency] across four populations of Raphanus raphanistrum monitored over a ten year period $\left(\Delta t=\frac{10}{1000000}=1.0 \times 10^{-5}\right)$.

\begin{tabular}{|c|c|c|c|c|}
\hline & $\begin{array}{c}\text { Generation 1 } \\
\text { Mean Frequency }\end{array}$ & $\begin{array}{c}\text { Generation } 10 \\
\text { Mean Frequency }\end{array}$ & darwins & darwins $\left(\times 10^{-3}\right)$ \\
\hline \multicolumn{5}{|c|}{ Molecular Marker: GPI } \\
\hline Population 1 & 0.25 & 0.07 & -127296.57 & -127.30 \\
\hline Population 2 & 0.25 & 0.06 & -142711.64 & -142.71 \\
\hline Population 3 & 0.25 & 0.12 & -73396.92 & -73.40 \\
\hline Population 4 & 0.25 & 0.055 & -151412.77 & -151.41 \\
\hline \multicolumn{5}{|c|}{ Molecular Marker: PGM } \\
\hline Population 1 & 0.25 & 0.26 & 3922.07 & 3.92 \\
\hline Population 2 & 0.25 & 0.16 & -44628.71 & -44.63 \\
\hline Population 3 & 0.25 & 0.25 & 0.00 & 0.00 \\
\hline Population 4 & 0.25 & 0.171 & -37979.74 & -37.98 \\
\hline \multicolumn{5}{|c|}{ Molecular Marker: White Flower Colour } \\
\hline Population 1 & 0.5 & 0.14 & -127296.57 & -127.30 \\
\hline Population 2 & 0.5 & 0.09 & -171479.84 & -171.48 \\
\hline Population 3 & 0.5 & 0.03 & -281341.07 & -281.34 \\
\hline Population 4 & 0.5 & 0.03 & -281341.07 & -281.34 \\
\hline
\end{tabular}




\section{Appendix 3 - Additional Life History Traits Measured}

\section{Methods}

\section{Data Collection}

Additional life history traits were measured including longest leaf length, stem diameter, and herbivory damage. Longest leaf length was measured using a ruler (Staples ${ }^{\circledR}$ brand, Newmarket, Ontario, Canada) and stem diameter using digital calipers (Treasna Instruments) at the point of cotyledon attachment. In 2015, when I noticed changes in the level of herbivory many plants were experiencing, I recorded herbivory once a month (June, July) using a visual damage scale ranging from zero to five, where: zero is perfectly intact and five is completely gone or eaten (Figure A3.1). In 2016, herbivory surveys were conducted once a month (June, July, and August).

\section{Statistical Analysis}

To test for differences between herbivory damage between years, I ran a non-parametric Mann-Whitney $U$ test due to the non-parametric distribution of the data between years. To test for an environment (year) by selection history (HW) by genotype (wild versus hybrid) effect on phenotype, I ran a full-factorial, linear mixed model MANOVA on the traits combined (i.e., longest leaf length, and stem diameter) and applied transformations, as necessary. To determine significant pair-wise differences, I first ran a full-factorial, mixed-model ANOVA for each phenotypic trait (using the aov function in R-Studio), followed by a post-hoc Tukey (package: car) that corrected for multiple comparisons of means (R-Studio ver. 1.0.1430).

To test for an environment (year) by selection history (HW) by genotype (wild vs hybrid effect on rates of divergence, I ran a mixed-model, repeated-measures MANOVA, with HW, genotype, and their interaction as the between-subjects effect and year and its interactions with HW and genotype as the within-subject effects (IBM SPSS Statistics 24, Chicago, USA). After determining a significant year interaction for all traits (i.e., leaf length and stem diameter), I ran separate full-factorial, repeated mixed-model ANOVA for each phenotypic trait (GLM function IBM SPSS Statistics 24, Chicago, USA). To determine significant pair-wise differences, I ran a post-hoc Tukey multiple comparisons of means test.

(continued) 


\section{Results}

Variation in Trait Evolution Between Years

Leaf and stem diameters were significantly larger in 2015 than 2016 across all watering by genotype interactions, except wild CS-evolved plants which did not vary (Table A3.1). In 2015, hybrid radish were not less resistant to herbivory (Mann-Whitney $U$-test; $\mathrm{z}=-1.63$, $\mathrm{p}=0.052$, one sided) than wild radish, with no differences in herbivory levels between genotypes in 2016. There were no significant differences between herbivory levels with respect to historical watering treatments and its interaction with genotype in 2015 and 2016.

\section{Consistency of Divergence Rates of $G_{5}$ Plants Between Years}

Leaf length and stem diameter divergence rates evolved in similar directions but at varying rates. Wild and hybrid water-evolved plants evolved larger leaf lengths and thinner stem diameters faster in 2015 than 2016 relative to CS-wild and hybrid plants (Table A3.2). In 2015, leaf lengths of NR, CU, and DR plants did not evolve away from CS plant phenotypes. In 2016, however, leaf lengths evolved to be longer in NR and DR plants relative to CS plants. Stem diameter evolutionary rates varied between years based on the historical watering treatment. In 2015, NR plants evolved thinner stem diameters slower than 2016 NR plants relative to CS plants. Also in 2015, CU and DR plants rapidly evolved thinner stem diameters relative to CS plants. Whereas, in 2016, CU and DR plants rapidly evolved wider stem diameters relative to CS plants.

Lastly, to determine if biotype by watering history interactions affect the evolutionary rates in $\mathrm{G}_{5}$ plants between common garden years after five years of evolution, I compared the magnitude and direction of phenotypic divergence in wild and hybrid plants grown under three watering treatments relative to wild plants grown under control shelter environments (Table A3.2). Leaf length and stem diameter divergence rates were similar but to varying magnitudes in 2015 and 2016. In 2015, NR-, DR- wild plants, and DR-hybrid plants evolved longer leaves faster in 2016 than 2015 relative to CS-wild and hybrid plants, respectively. Similarly, NR-wild plants evolved smaller diameters relative to CS-wild plants faster in 2015 than 2016. Stem diameter divergence rates, however, were contradictory between 2015 and 2016 common garden years depending on the biotype by watering history interaction. In 2015, wild-CU and wild-DR 
plants quickly evolved smaller diameters compared to CS-wild phenotypes. While, in 2016, wild CU and DR plants quickly evolved larger stem diameters relative to CS-wild phenotypes.

\section{Divergence Rate Correlations Between Years}

Overall, from my simple-linear regression analyses, I found 2015 leaf length divergence rates can only marginally predict 2016 leaf length rates but stem diameter (Table A3.3). Within genotypes 2015 hybrid stem diameter divergence rates can predict 2016 hybrid stem diameter rates but not leaf length divergence rates (Table A3.3). Plants from double rain (DR) selection histories in 2015 can confidently predict leaf length and stem diameter divergence rates in 2016. Finally, genotype $\times$ watering history interactions have varying predictability strengths (Table A2.3). Leaf length divergence rates in NR-hybrid, and NR-wild plants can confidently be predicted between years, with a marginal correlation among DR-hybrid plants. Similarly, 2015 stem diameter divergence rates of NR-hybrid, CU-hybrid, and CU-wild plants can confidently predict 2016 NR-hybrid stem diameter divergence rates.

(continued) 
Table A3.1: Mixed model ANOVA of mean trait plasticity [ a) longest leaf length (Box-Cox transformation $\lambda=0.4595443, \mathrm{n}=650), \mathrm{b}$ ) stem diameter (Box-Cox transformation $\lambda=$

$0.05673421, \mathrm{n}=650$ ] between years in response to biotype (wild versus hybrid), historic watering treatment (WH: no rain, control shelter, control unsheltered, and double rain), and their interaction. A $\chi^{2}$ significance test was run comparing the model with and without the block factor (i.e, measuring the significance of block in the model). (a) The model measuring longest leaf length had a significant block effect $\left[\chi_{(\mathrm{df}=1)}^{2}=88.50, \mathrm{p}<0.001\right]$. (b) The model measuring stem diameter had a significant block effect $\left[\chi_{(\mathrm{df}=1)}^{2}=27.96, \mathrm{p}<0.001\right]$. The fixed effects models compute the F-statistic using the mean square error of each model (presented as Error in the table). F-statistics are presented to indicate significant differences: $\mathrm{ns}, \mathrm{P}>0.10 ;+, \mathrm{P}<0.10 ; *, \mathrm{P}$ $<0.05 ; * *, \mathrm{P}<0.01 ; * * *, \mathrm{P}<0.001$.

\begin{tabular}{|c|c|c|c|}
\hline $\begin{array}{c}\text { Response \& } \\
\text { Parameter }\end{array}$ & df & Mean Square & F-Statistic \\
\hline \multicolumn{4}{|c|}{ a) Leaf Length (Box-cox transformation $\lambda=0.4595443, n=650$ ) } \\
\hline Genotype $(\mathrm{G})$ & 1 & 450.11 & $57.97^{* * *}$ \\
\hline $\begin{array}{l}\text { Watering History } \\
\text { (WH) }\end{array}$ & 3 & 18.26 & $2.35^{+}$ \\
\hline Year $(Y)$ & 1 & 589.01 & $75.85^{* * *}$ \\
\hline $\mathrm{WH} \times \mathrm{G}$ & 3 & 120.61 & $15.53^{* * *}$ \\
\hline $\mathrm{WH} \times \mathrm{Y}$ & 3 & 2.38 & $0.31^{\mathrm{ns}}$ \\
\hline $\mathrm{G} \times \mathrm{Y}$ & 1 & 392.99 & $50.61^{* * *}$ \\
\hline $\mathrm{WH} \times \mathrm{G} \times \mathrm{Y}$ & 3 & 97.37 & $12.54^{* * *}$ \\
\hline Error & 616 & 7.77 & - \\
\hline \multicolumn{4}{|c|}{ b) Stem Diameter (Box-Cox transformation $\lambda=-0.05673421, \mathrm{n}=650$ ) } \\
\hline G & 1 & 14.04 & $121.72^{* * *}$ \\
\hline WH & 3 & 0.26 & $2.32^{+}$ \\
\hline Y & 1 & 11.72 & $101.64^{* * *}$ \\
\hline $\mathrm{WH} \times \mathrm{G}$ & 3 & 3.26 & $28.30^{* * *}$ \\
\hline $\mathrm{WH} \times \mathrm{Y}$ & 3 & 0.06 & $0.48^{\mathrm{ns}}$ \\
\hline $\mathrm{G} \times \mathrm{Y}$ & 1 & 3.41 & $29.56^{* * *}$ \\
\hline $\mathrm{WH} \times \mathrm{G} \times \mathrm{Y}$ & 3 & 2.53 & $21.93^{* * *}$ \\
\hline Error & 617 & 0.12 & - \\
\hline
\end{tabular}


Table A3.2: Repeated measures fixed-factor ANOVA of divergence rates of a) longest leaf length and b) stem diameter and their response to genotype (wild versus hybrid), watering history (WH: no rain, control unsheltered, and double rain), and their interaction, with Year as the repeated measure. In both models, 27 populations were considered when testing the between subject's effects. The error term used for within subject's and between subject's effects are listed in the ANOVA tables under each respective category along with each model's sample size (n). F-statistics are presented to indicate significant differences: $\mathrm{ns}, \mathrm{P}>0.10 ;+\mathrm{P}<0.10 ; *, \mathrm{P}<0.05$; $* *, \mathrm{P}<0.01 ; * * *, \mathrm{P}<0.001$.

\begin{tabular}{|c|c|c|c|c|c|c|c|}
\hline \multirow{2}{*}{$\begin{array}{c}\text { Response } \\
\& \\
\text { Parameter }\end{array}$} & \multicolumn{3}{|c|}{ Within Subject's Effects } & \multirow{2}{*}{$\begin{array}{c}\text { Response } \\
\& \\
\text { Parameter }\end{array}$} & \multicolumn{3}{|c|}{ Between Subject's Effects } \\
\hline & df & $\begin{array}{c}\text { Mean } \\
\text { Square }\end{array}$ & $\begin{array}{c}\mathbf{F} \\
\text { Statistic }\end{array}$ & & df & $\begin{array}{c}\text { Mean } \\
\text { Square }\end{array}$ & $\begin{array}{c}\mathbf{F} \\
\text { Statistic }\end{array}$ \\
\hline \multicolumn{8}{|c|}{ a) Longest Leaf Length Divergence Rate $(\mathrm{n}=\mathbf{2 5 7})$} \\
\hline Year $(\mathrm{Y})$ & 1 & 0.46 & $6.68^{*}$ & $\begin{array}{l}\text { Watering } \\
\text { History } \\
(\mathrm{WH})\end{array}$ & 2 & 0.43 & $1.05^{\mathrm{ns}}$ \\
\hline $\mathrm{Y} \times \mathrm{WH}$ & 2 & 0.06 & $0.91^{\mathrm{ns}}$ & $\begin{array}{l}\text { Genotype } \\
\text { (G) }\end{array}$ & 1 & 2.72 & $6.68^{*}$ \\
\hline $\mathrm{Y} \times \mathrm{G}$ & 1 & 3.25 & $47.68^{* * *}$ & $\mathrm{WH} \times \mathrm{G}$ & 2 & 0.63 & $1.53^{\mathrm{ns}}$ \\
\hline $\begin{array}{l}\mathrm{Y} \times \mathrm{WH} \times \\
\mathrm{G}\end{array}$ & 2 & 0.03 & $0.49^{\text {ns }}$ & Error & 21 & 0.41 & \\
\hline $\begin{array}{l}\text { Error } \\
\text { (Year) }\end{array}$ & 224 & 0.07 & & & & & \\
\hline \multicolumn{8}{|c|}{ b) Stem Diameter Divergence Rate $(n=257)$} \\
\hline Y & 1 & 0.08 & $1.49^{\mathrm{ns}}$ & WH & 2 & 0.36 & $0.73^{\text {ns }}$ \\
\hline $\mathrm{Y} \times \mathrm{WH}$ & 2 & 0.03 & $0.59^{\mathrm{ns}}$ & G & 1 & 0.17 & $0.34^{\mathrm{ns}}$ \\
\hline $\mathrm{Y} \times \mathrm{G}$ & 1 & 2.14 & $40.61^{* * * *}$ & $\mathrm{WH} \times \mathrm{G}$ & 2 & 0.40 & $1.41^{\mathrm{ns}}$ \\
\hline $\begin{array}{l}\mathrm{Y} \times \mathrm{WH} \times \\
\mathrm{G}\end{array}$ & 2 & 0.07 & $1.25^{\mathrm{ns}}$ & Error & 21 & 0.50 & \\
\hline $\begin{array}{l}\text { Error } \\
\text { (Year) }\end{array}$ & 224 & 0.05 & & & & & \\
\hline
\end{tabular}


Table A3.3: Rate of divergence regression analyses for several traits (longest leaf length and stem diameter) between 2015 and 2016 common garden data response to year $(2015,2016)$, genotype (wild versus hybrid), historical watering environment (HW: no rain, control shelter, control unsheltered, and double rain), and their interaction. Correlation coefficients are presented with beta-values in parentheses. To indicate significant differences: NA; not applicable; ns, P > $0.10 ;+, \mathrm{P}<0.10 ; *, \mathrm{P}<0.05 ; * *, \mathrm{P}<0.01 ; * * *, \mathrm{P}<0.001$.

\begin{tabular}{|c|c|c|}
\hline \multirow{3}{*}{ Parameters } & \multicolumn{2}{|c|}{$\begin{array}{c}\text { Correlation Coefficient } r^{2} \text { and } \beta \text {-values of Rate of } \\
\text { Divergence }(h)\end{array}$} \\
\hline & \multicolumn{2}{|c|}{ Responses $-\mathbf{r}^{2}(\beta)$} \\
\hline & Longest Leaf Length (cm) & Stem Diameter (mm) \\
\hline \multicolumn{3}{|l|}{ Year } \\
\hline 2015 vs. 2016 & $0.027(0.16)^{+}$ & $0.00(0.02)^{\mathrm{ns}}$ \\
\hline \multicolumn{3}{|l|}{ Genotype (G) } \\
\hline Wild & $0.038(0.19)^{\mathrm{ns}}$ & $0(0.02)^{\mathrm{ns}}$ \\
\hline Hybrid & $0.02(0.16)^{\mathrm{ns}}$ & $0.23(0.48)^{* * * *}$ \\
\hline \multicolumn{3}{|l|}{ Historical Watering } \\
\hline \multicolumn{3}{|l|}{ Treatment (WH) } \\
\hline No Rain (NR) & $0.24(0.06)^{\mathrm{ns}}$ & $0.13(0.02)^{\mathrm{ns}}$ \\
\hline Control Unsheltered (CU) & $0.04(0.19)^{\mathrm{ns}}$ & $0.08(0.28)^{+}$ \\
\hline Double Rain (DR) & $0.12(0.35)^{*}$ & $0.12(0.35)^{*}$ \\
\hline \multicolumn{3}{|l|}{$\mathbf{W H} \times \mathbf{G}$} \\
\hline NR-Wild & $0.58(0.76)^{* * *}$ & $0.14(0.37)^{\mathrm{ns}}$ \\
\hline CU-Wild & $0.02(0.12)^{\mathrm{ns}}$ & $0.76(0.87)^{* * *}$ \\
\hline DR-Wild & $0.16(0.40)^{+}$ & $0.05(0.21)^{\mathrm{ns}}$ \\
\hline NR-Hybrid & $0.23(0.48)^{*}$ & $0.34(0.59)^{* *}$ \\
\hline CU-Hybrid & $0.09(0.31)^{\mathrm{ns}}$ & $0.25(0.50)^{*}$ \\
\hline DR-Hybrid & $0.14(0.37)^{+}$ & $0.00(0.06)^{\mathrm{ns}}$ \\
\hline
\end{tabular}


(A)

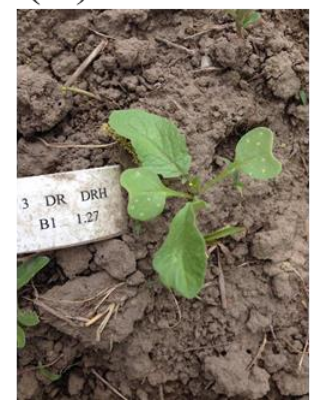

(B)

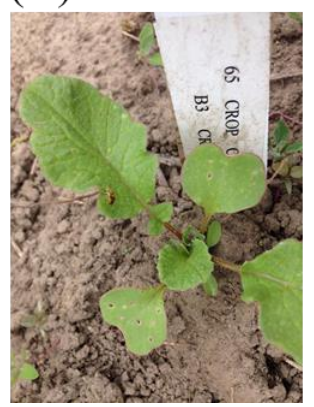

(C)

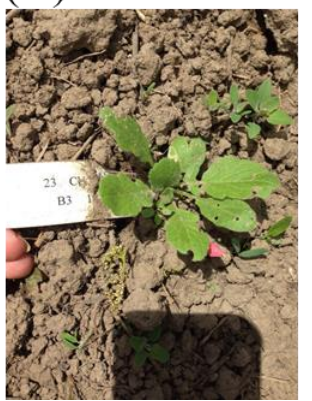

(D)

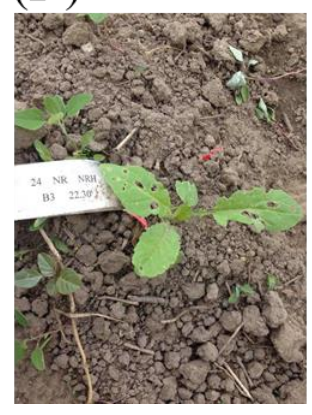

(E)

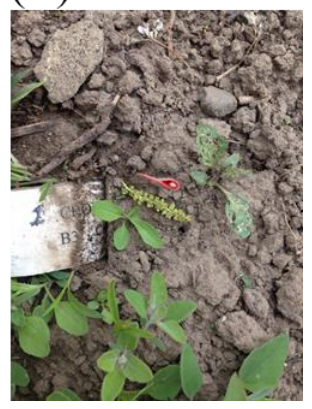

Figure A3.1: A visual scale of herbivory damage that ranges from zero to five; zero represents intact conditions (i.e., no herbivory) and five represents complete destruction (i.e., completely eaten). Plants represent damage level: (A) 0.5, (B) 1, (C) 2, (D) 2.5, and (E) 4. 


\section{Appendix 4 - Reaction Norm Repeated-Measures Analysis}

Table A4.1: Repeated-measures analysis results of reaction norms on four phenotypic traits [ a) days to emergence (Box-Cox transformation $\lambda=-1.116995, \mathrm{n}=328$ ), $\mathrm{b}$ ) days to flowering (BoxCox transformation $\lambda=0.1505881, \mathrm{n}=327)$, c) longest leaf length (Box-Cox transformation $\lambda=$ 0.310807, $\mathrm{n}=328$ ), and d) stem diameter (Box-Cox transformation $\lambda=-0.02991597, \mathrm{n}=328$ )] Phenotypic traits were tested in response to Year (Y: 2015, 2016), a combine variable of historic watering treatment and genotype (HWG: no rain-wild, control shelter-wild, control unshelteredwild, double rain-wild, no rain-hybrid, control shelter-hybrid, control unsheltered-hybrid, and double rain-hybrid), and their interaction. Population is used as the repeated-measures between years. F-statistics are presented to indicate significant differences: $\mathrm{ns}, \mathrm{P}>0.10 ;+, \mathrm{P}<0.10 ;{ }^{*}, \mathrm{P}$ $<0.05 ; * *, \mathrm{P}<0.01 ; * * *, \mathrm{P}<0.001$.

\begin{tabular}{ll|lc}
\hline \multirow{2}{*}{$\begin{array}{c}\text { Responses \& } \\
\text { Parameters }\end{array}$} & Within Subject's Effects & $\begin{array}{c}\text { Responses \& } \\
\text { Parameters }\end{array}$ & $\begin{array}{c}\text { Between Subject's Effects - } \\
\end{array}$ \\
\cline { 2 - 3 } & df (n,d) & & Error: Population \\
\hline
\end{tabular}

a) Days to emergence

\begin{tabular}{lll|lll}
\hline & & $\begin{array}{l}\text { Historic } \\
\text { Watering }\end{array}$ & & \\
Year (Y) & 1,291 & $0.926^{\mathrm{ns}}$ & $\begin{array}{l}\text { Treatment- } \\
\text { Genotype }\end{array}$ & 7,21 & $1.69^{\mathrm{ns}}$ \\
& & (HWG) & \\
$\mathrm{Y} \times \mathrm{HWG}$ & 7,291 & $0.975^{\mathrm{ns}}$ & & \\
\hline
\end{tabular}

b) Days to flowering

\begin{tabular}{lcc|lll}
\hline $\mathrm{Y}$ & 1,290 & $15.50^{* * *}$ & $\mathrm{Y}$ & 1,20 & $7.52^{*}$ \\
$\mathrm{Y} \times \mathrm{HWG}$ & 7,290 & $1.06^{\mathrm{ns}}$ & $\mathrm{HWG}$ & 7,20 & $5.60^{* *}$ \\
\hline
\end{tabular}

c) Longest Leaf Length

\begin{tabular}{lcc|lcc}
\hline $\mathrm{Y}$ & 1,291 & $22.66^{* * *}$ & HWG & 7,21 & $10.59^{* * *}$ \\
$\mathrm{Y} \times \mathrm{HWG}$ & 7,291 & $0.42^{\text {ns }}$ & & & \\
\hline
\end{tabular}

\begin{tabular}{lcc|lcc}
\hline d) Stem Diameter & \multicolumn{5}{l}{} \\
\hline $\mathrm{Y}$ & 1,291 & $17.02^{* * *}$ & HWG & 7,21 & $14.35^{* * *}$ \\
$\mathrm{Y} \times \mathrm{HWG}$ & 7,291 & $0.299^{\text {ns }}$ & & & \\
\hline
\end{tabular}




\section{Appendix 5 - Divergence Rate Correlations Between Years for Genotype, Historical Watering Environment}

\section{Results}

Within watering-selection histories, NR- and DR-evolved 2015 white colour frequency rates can confidently predict 2016 rates but not among CU-evolved plants. Within genotypes, hybrid or wild population divergence rates of days to flowering in 2015 could confidently predict 2016 rates, as well. Similarly, values from 2015 can confidently predict days to flowering divergence rates (but not days to emergence) for populations from all watering histories (NR, CU, DR) in 2016 (Table A5.1). Divergence rates of emergence time cannot be predicted between years across any genotype $\times$ watering condition. Further, divergence rates in 2015 only sometimes predicted 2016 rates, depending on the experimental context of genotype and watering history interactions (Table A5.1). In 2015, flowering divergence rates of NR-hybrid plants can confidently predict 2016 NR-hybrid flowering divergence rates. Similarly, I found that 2015 NR-wild plant flowering divergence rates can confidently predict 2016 NR-wild plant flowering divergence rates. Further, 2015 CU-hybrid plant flowering divergence rates were correlated with those in 2016. However, DR-hybrid flowering divergence rates were not correlated among years. Finally, the divergence rates of flowering time of wild DR and CU populations were correlated between years, but was not for wild CU populations.

(continued) 
Table A5.1: Rate of divergence regression analyses for several traits (days to emergence, days to flowering, and white colour frequency) between 2015 and 2016 common garden data response to genotype (wild versus hybrid), historical watering environment (HW: no rain, control shelter, control unsheltered, and double rain), and their interaction. Correlation coefficients are presented with beta-values in parentheses. To indicate significant differences: NA; not applicable; ns, P > $0.10 ;+, \mathrm{P}<0.10 ; *, \mathrm{P}<0.05 ; * *, \mathrm{P}<0.01 ; * * *, \mathrm{P}<0.001$.

Correlation Coefficient $r^{2}$ and $\beta$-values of Rate of Divergence $(h)$

\begin{tabular}{|c|c|c|c|}
\hline \multirow[b]{2}{*}{ Parameters } & \multicolumn{3}{|c|}{ Responses $-\mathbf{r}^{2}(\beta)$} \\
\hline & Days to emergence & Days to flowering & $\begin{array}{c}\text { White flower colour } \\
\text { frequency }\end{array}$ \\
\hline \multicolumn{4}{|l|}{ Genotype (G) } \\
\hline$\overline{\text { Wild }}$ & $0.00(0.02)^{\mathrm{ns}}$ & $0.39(0.62)^{* * *}$ & $\mathrm{NA}$ \\
\hline Hybrid & $0.01(0.11)^{\mathrm{ns}}$ & $0.28(0.53)^{* * *}$ & \\
\hline \multicolumn{4}{|l|}{ Historical Watering } \\
\hline \multicolumn{4}{|l|}{ Treatment (HW) } \\
\hline No Rain (NR) & $0^{\mathrm{ns}}$ & $0.90^{* * * *}$ & $0.35(0.59)^{* *}$ \\
\hline $\begin{array}{l}\text { Control Unsheltered } \\
\text { (CU) }\end{array}$ & $0.00(0.02)^{\mathrm{ns}}$ & $0.25(0.50)^{* * *}$ & $0.00(0.04)^{\mathrm{ns}}$ \\
\hline Double Rain (DR) & $0.02(0.14)^{\mathrm{ns}}$ & $0.16(0.40)^{* *}$ & $0.29(0.53)^{* *}$ \\
\hline \multicolumn{4}{|l|}{$\mathbf{H W} \times \mathbf{G}$} \\
\hline NR-Wild & $0.03(0.16)^{\mathrm{ns}}$ & $0.77(0.88)^{* * *}$ & \\
\hline CU-Wild & $0.00(0.03)^{\mathrm{ns}}$ & $0.18(0.42)^{\mathrm{ns}}$ & \\
\hline DR-Wild & $0.03(0.17)^{\mathrm{ns}}$ & $0.30(0.55)^{*}$ & \\
\hline NR-Hybrid & $0.01(0.09)^{\mathrm{ns}}$ & $0.30(0.51)^{*}$ & \\
\hline CU-Hybrid & $0.00(0.01)^{\mathrm{ns}}$ & $0.38(0.62)^{* * *}$ & \\
\hline DR-Hybrid & $0.04(0.19)^{\mathrm{ns}}$ & $0.06(0.24)^{\mathrm{ns}}$ & \\
\hline
\end{tabular}




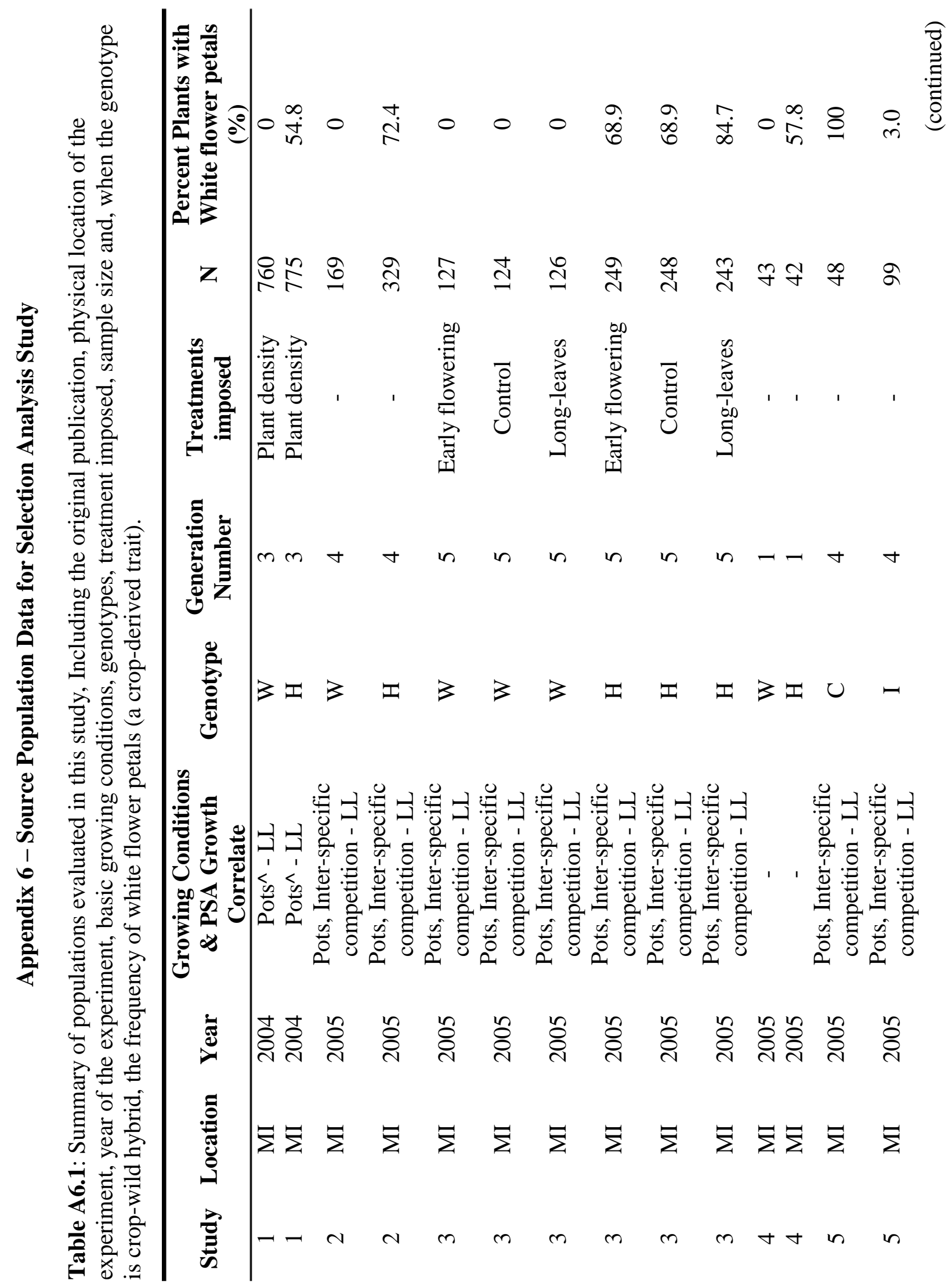




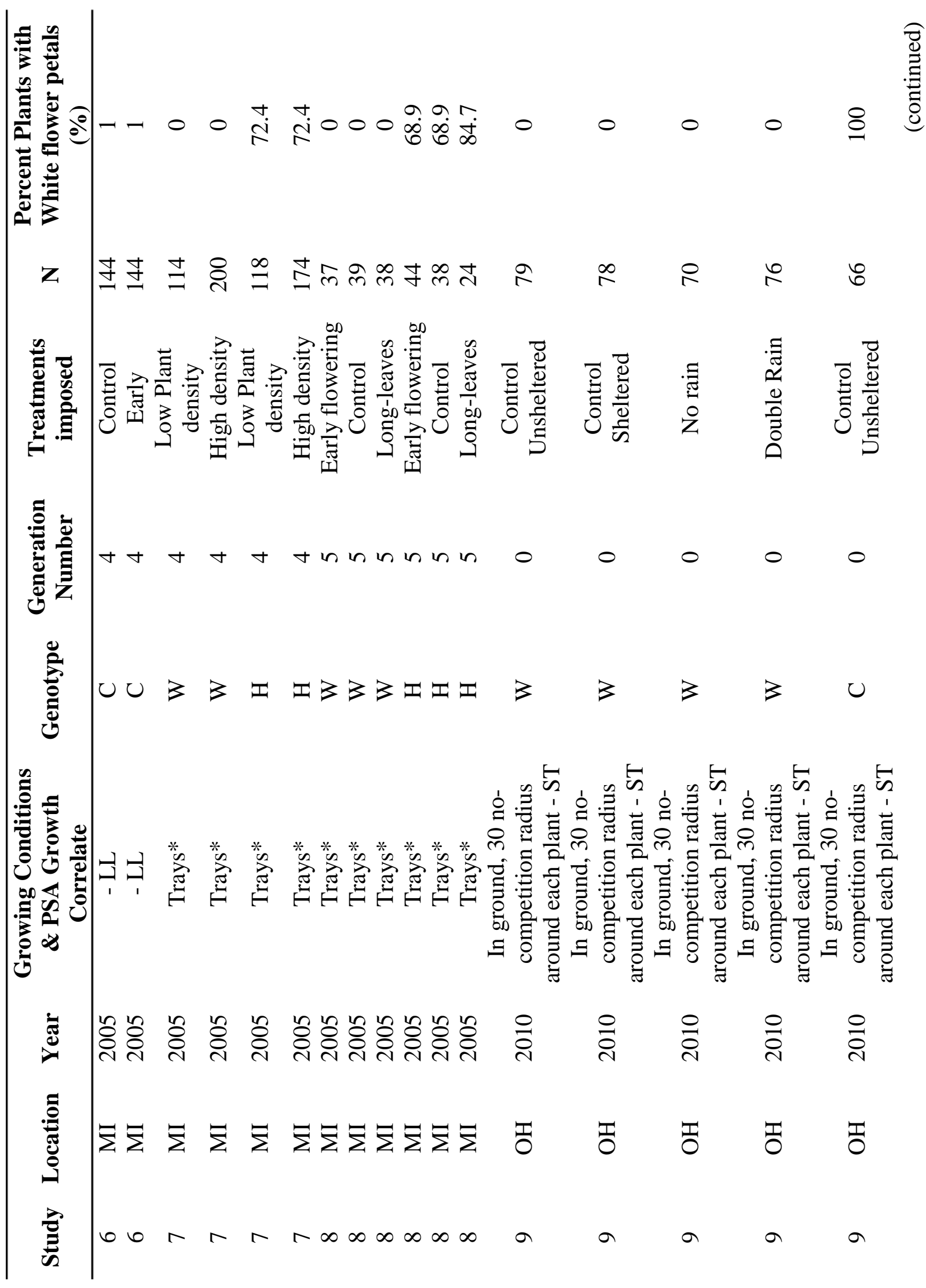




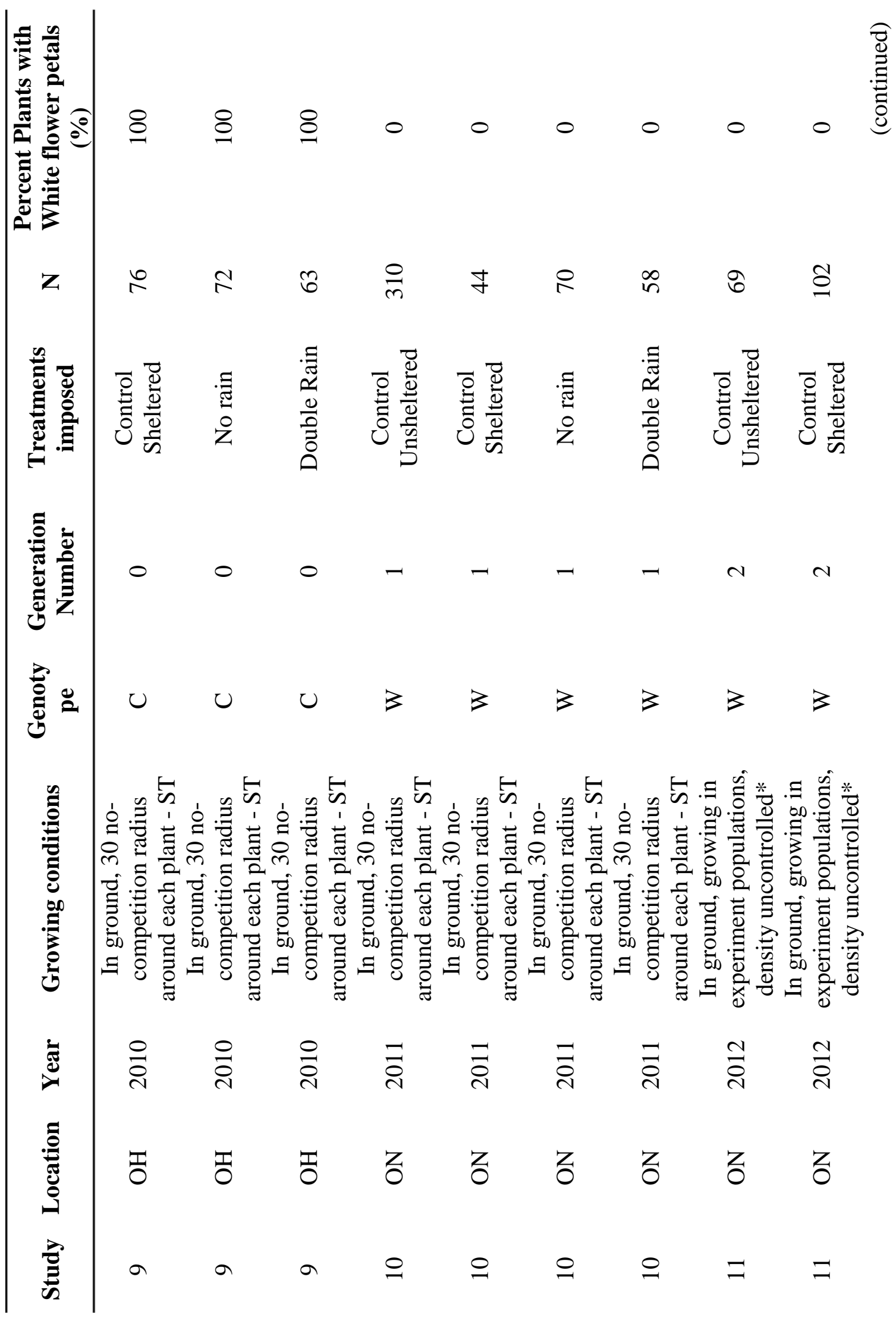




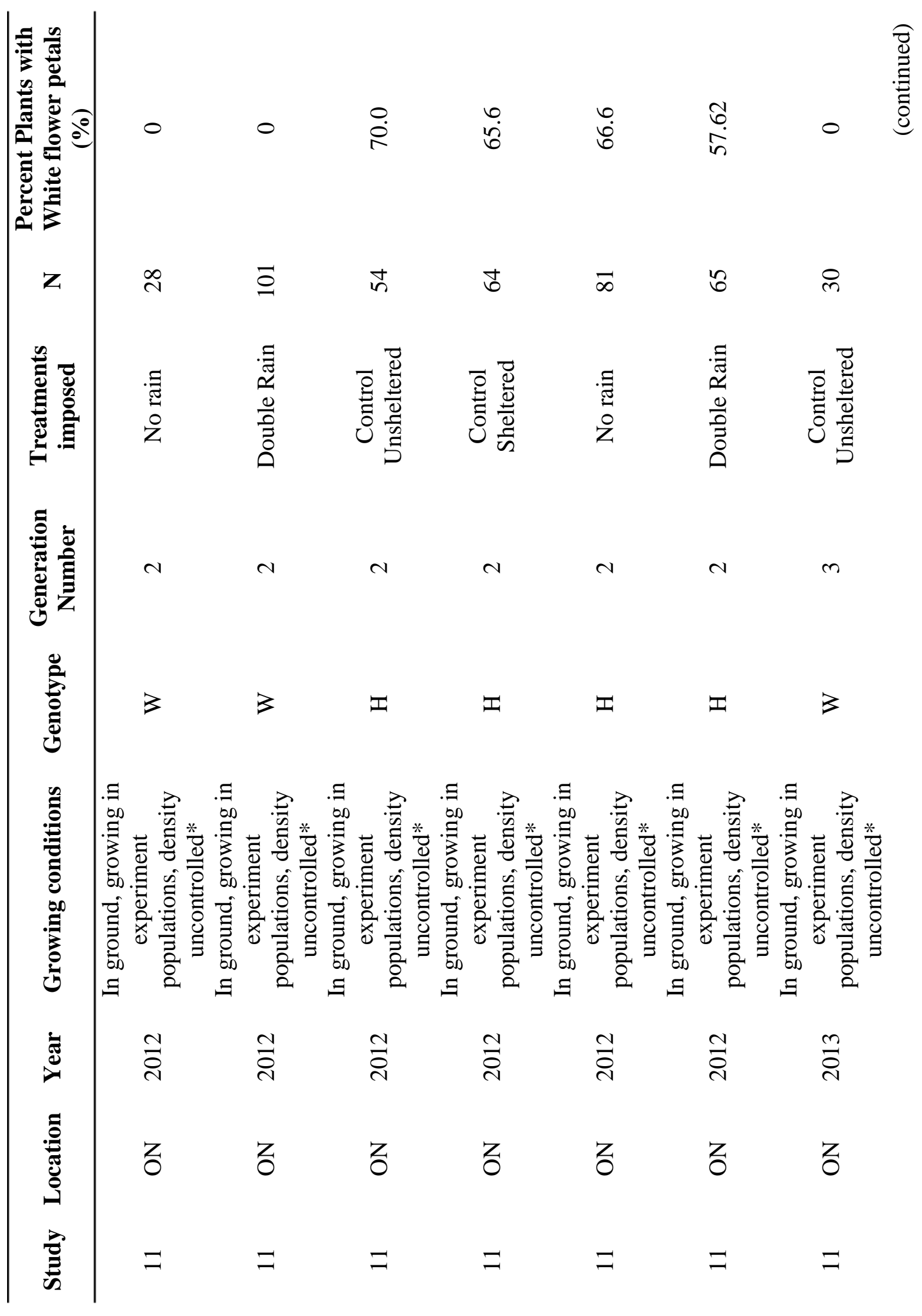




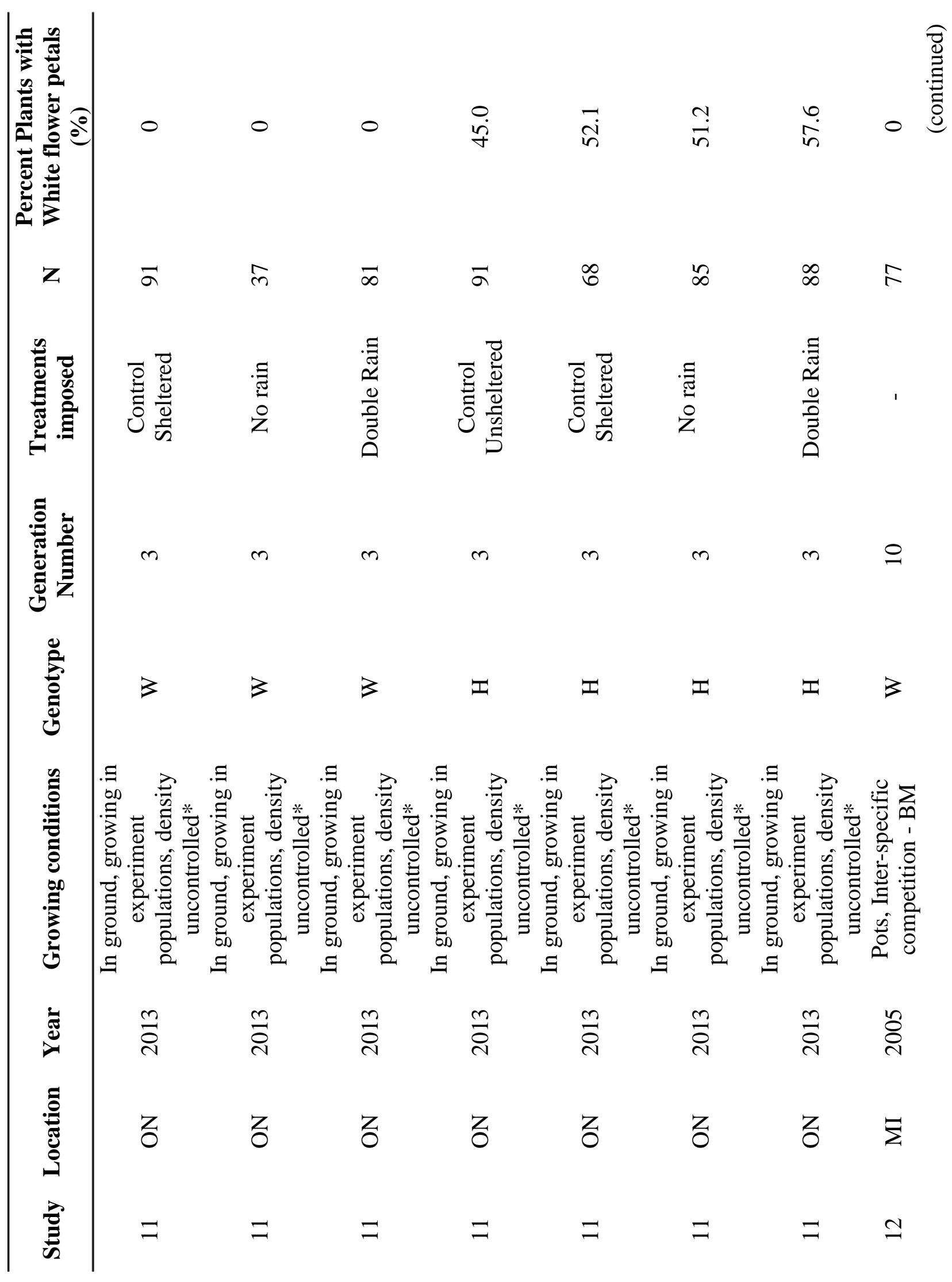




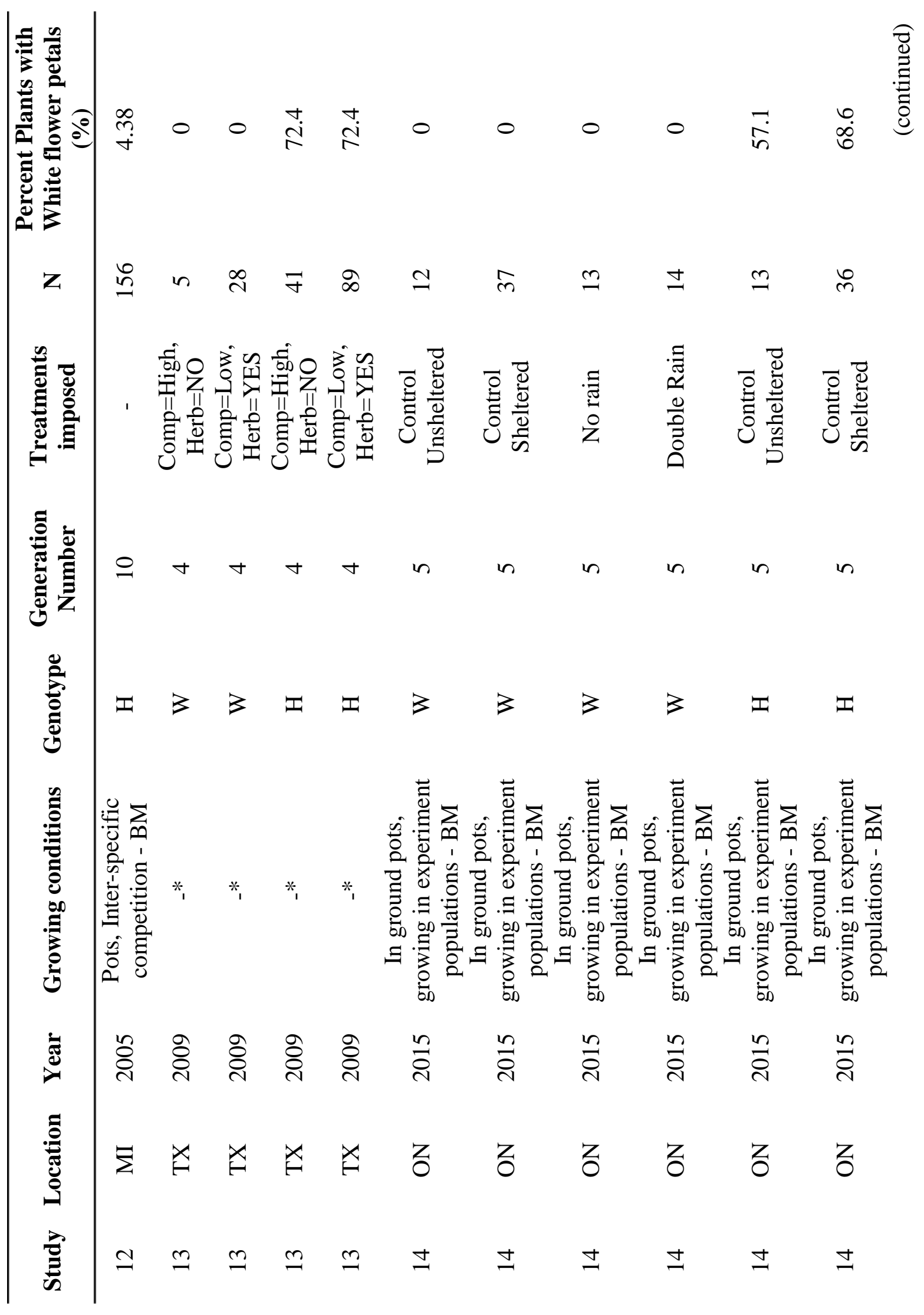




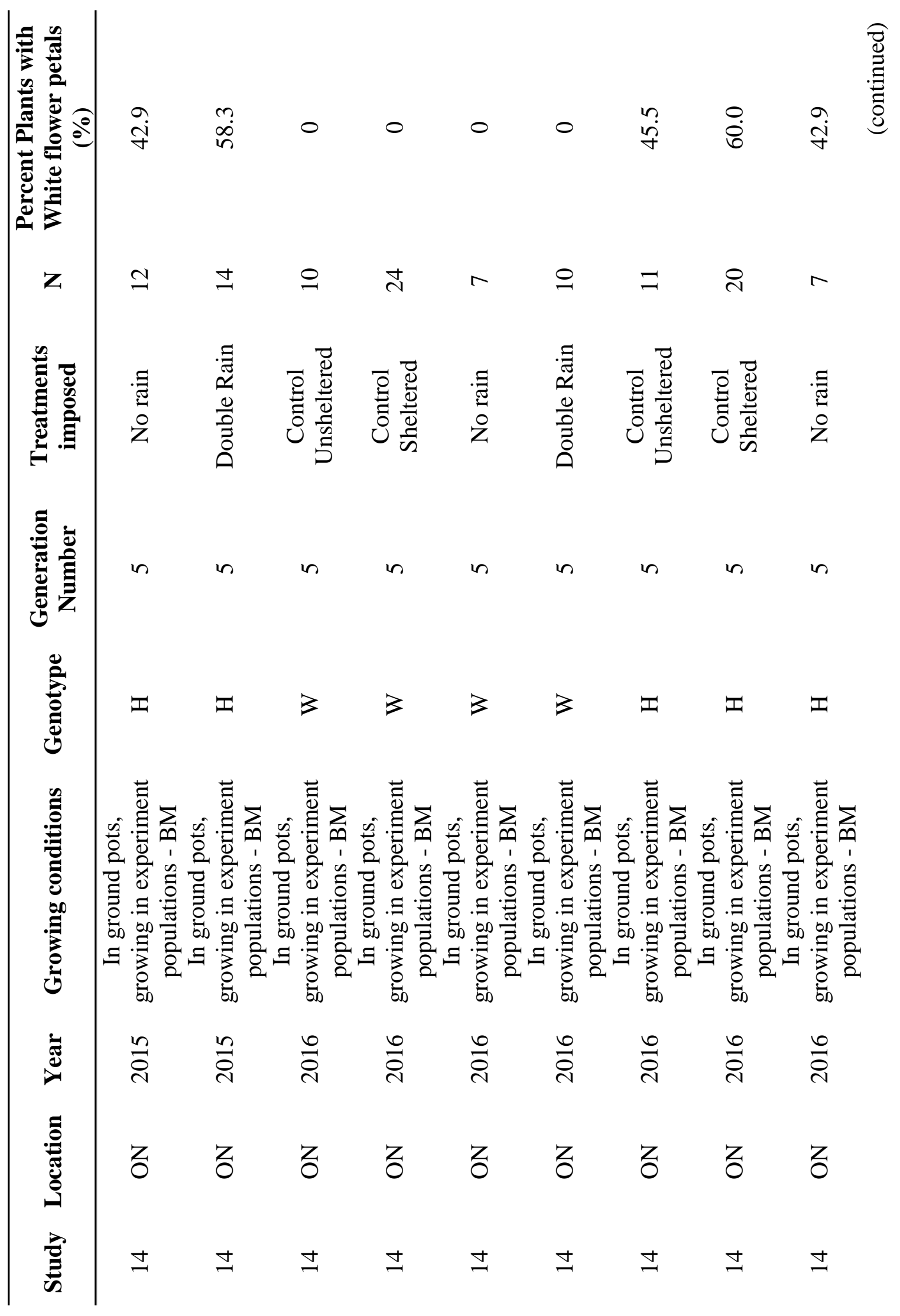




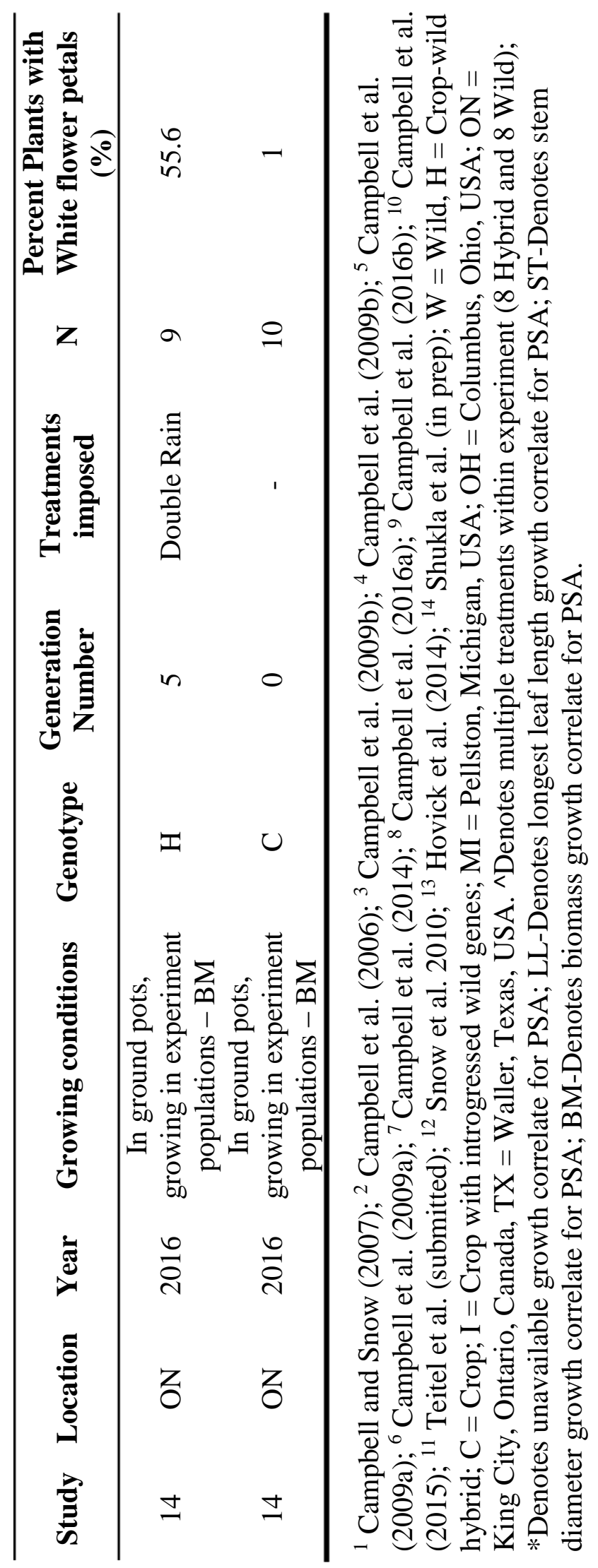




\section{Appendix 7 - Error Estimates on Selection Gradient Values in Michigan Populations}
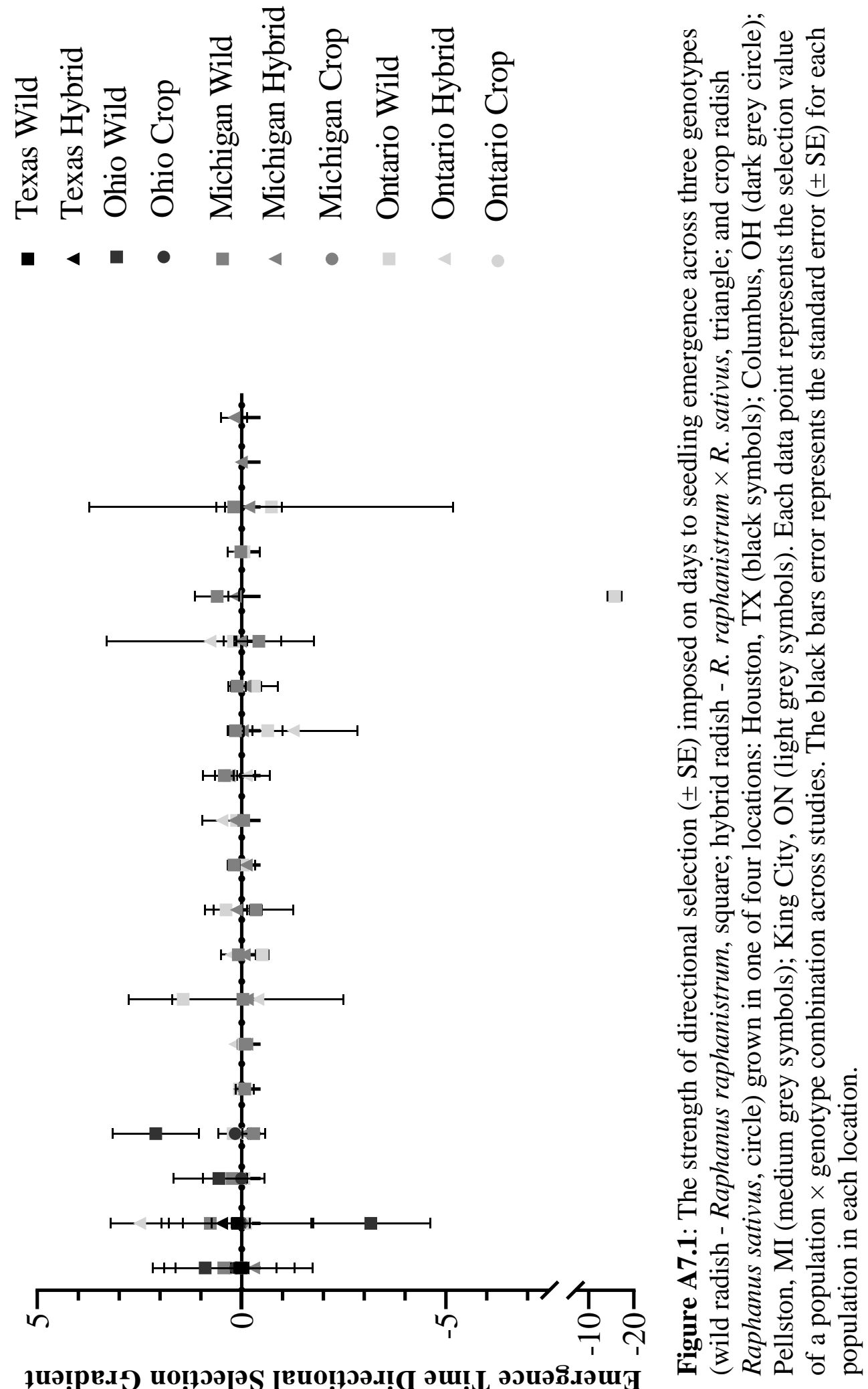


\section{References}

Abbamondi, G. R., G. Tommonaro, N. Weyens, S. Thijs, W. Sillen, P. Gkorezis, C. Iodice, W. de Melo Rangel, B. Nicolaus, and J. Vangronsveld. 2016. Plant growth-promoting effects of rhizospheric and endophytic bacteria associated with different tomato cultivars and new tomato hybrids. Chemical and Biological Technologies in Agriculture 3:1.

Abbott, R. J. 1992. Plant invasions, interspecific hybridization and the evolution of new plant taxa. Trends in Ecology \& Evolution 7:401-405.

Abbott, R. J., J. K. James, R. I. Milne, and A. C. Gillies. 2003. Plant introductions, hybridization and gene flow. Philosophical Transactions of the Royal Society of London B: Biological Sciences 358:1123-1132.

Abdel-Ghani, A. H., H. K. Parzies, A. Omary, and H. H. Geiger. 2004. Estimating the outcrossing rate of barley landraces and wild barley populations collected from ecologically different regions of Jordan. Theoret. Appl. Genetics 109:588-595.

Adamowski, J., K. Adamowski, and A. Prokoph. 2013. Quantifying the spatial temporal variability of annual streamflow and meteorological changes in eastern Ontario and southwestern Quebec using wavelet analysis and GIS. Journal of Hydrology 499:27-40.

Agriculture and Agro-Food Canada (AAFC). 2016. Reduced-Risk Strategy for Integrated Weed Management in Field Vegetables.

Albert, M. E., C. M. D'Antonio, and K. A. Schierenbeck. 1997. Hybridization and introgression in Carpobrotus spp. (Aizoaceae) in California. I. Morphological evidence. American Journal of Botany 84:896-904.

Alberti, M., C. Correa, J. M. Marzluff, A. P. Hendry, E. P. Palkovacs, K. M. Gotanda, V. M. Hunt, T. M. Apgar, and Y. Zhou. 2017. Global urban signatures of phenotypic change in animal and plant populations. Proceedings of the National Academy of Sciences 114:8951-8956.

Alseekh, S., H. Tong, F. Scossa, Y. Brotman, F. Vigroux, T. Tohge, I. Ofner, D. Zamir, Z. Nikoloski, and A. R. Fernie. 2017. Canalization of Tomato Fruit Metabolism. The Plant Cell 29:2753-2765.

Amasino, R. M. and S. D. Michaels. 2010. The timing of flowering. Plant Physiology 154:516520.

Anderson, E. and G. L. Stebbins. 1954. Hybridization as an evolutionary stimulus. Evolution 8:378-388.

Anderson, E. and G. L. Stebbins Jr. 1954. Hybridization as an evolutionary stimulus. Evolution 8:378-388.

Anderson, J. T., J. H. Willis, and T. Mitchell-Olds. 2011. Evolutionary genetics of plant adaptation. Trends in Genetics 27:258-266.

Arnold, M. L. 1994. Natural hybridization and Louisiana irises. BioScience 44:141-147.

Arnold, M. L. and S. A. Hodges. 1995. Are natural hybrids fit or unfit relative to their parents? Trends in ecology \& evolution 10:67-71.

Arnold, M. L. and N. H. Martin. 2010. Hybrid fitness across time and habitats. Trends in Ecology \& Evolution 25:530-536.

Ashworth, M. B., M. J. Walsh, K. C. Flower, and S. B. Powles. 2016. Recurrent selection with reduced 2, 4-D amine doses results in the rapid evolution of 2, 4-D herbicide resistance in wild radish (Raphanus raphanistrum L.). Pest management science 72:2091-2098. 
Baack, E. J., Y. Sapir, M. A. Chapman, J. M. Burke, and L. H. Rieseberg. 2008. Selection on domestication traits and quantitative trait loci in crop-wild sunflower hybrids. Molecular Ecology 17:666-677.

Baker, H. G. 1974. The Evolution of Weeds. Annual Review of Ecology and Systematics 5:1-24.

Baker, H. G., H. Baker, and G. Stebbins. 1965. Characteristics and modes of origin of weeds. The genetics of colonizing species.:147-168.

Baker, N. R. and E. Rosenqvist. 2004. Applications of Chlorophyll Fluorescence Can Improve Crop Production Strategies: An Examination of Future Possibilities. Journal of Experimental Botany 55:1607-1621.

Baldini, M. and G. P. Vannozzi. 1999. Yield relationships under drought in sunflower genotypes obtained from a wild population and cultivated sunflowers in rain-out shelter in large pots and field experiments. Helia (Yugoslavia).

Balota, M. and H. K. Lichtenthaler. 1999. Red chlorophyll fluorescence as an ecophysiological method to assess the behaviour of wheat genotypes under drought and heat. Cereal Research Communications:179-187.

Barrett, S. C. 2014. Evolution of mating systems: outcrossing versus selfing. Pp. 356-362. Princeton University Press Princeton.

Barrett, S. C. H. and L. D. Harder. 1996. Ecology and evolution of plant mating. Trends in Ecology \& Evolution 11:73-79.

Barrick, J. E., D. S. Yu, S. H. Yoon, H. Jeong, T. K. Oh, D. Schneider, R. E. Lenski, and J. F. Kim. 2009. Genome evolution and adaptation in a long-term experiment with Escherichia coli. Nature 461:1243.

Barton, N. H. 2001. The role of hybridization in evolution. Molecular Ecology 10:551-568.

Barton, N. H. and G. M. Hewitt. 1985. Analysis of hybrid zones. Annual Review of Ecology and Systematics 16:113-148.

Bartsch, D., M. Lehnen, J. Clegg, M. Pohl-Orf, I. Schuphan, and N. C. Ellstrand. 1999. Impact of gene flow from cultivated beet on genetic diversity of wild sea beet populations. Molecular Ecology 8:1733-1741.

Beckie, H. J., R. E. Blackshaw, R. Low, L. M. Hall, C. A. Sauder, S. Martin, R. N. Brandt, and S. W. Shirriff. 2013. Glyphosate-and acetolactate synthase inhibitor-resistant kochia (Kochia scoparia) in western Canada. Weed Science 61:310-318.

Becklin, K. M., J. T. Anderson, L. M. Gerhart, S. M. Wadgymar, C. A. Wessinger, and J. K. Ward. 2016. Examining plant physiological responses to climate change through an evolutionary lens. Plant physiology 172:635-649.

Berecha, G., R. Aerts, K. Vandepitte, S. Van Glabeke, B. Muys, I. Roldán-Ruiz, and O. Honnay. 2014. Effects of forest management on mating patterns, pollen flow and intergenerational transfer of genetic diversity in wild Arabica coffee (Coffea arabica L.) from Afromontane rainforests. Biological Journal of the Linnean Society 112:76-88.

Bhatti, M., P. Cocks, S. Bennett, and A. Malik. 2016. Adaptive significance of within-site variation in morphological and reproductive traits of naturalized wild radish (Raphanus raphanistrum) populations in South-Western Australia. International Journal of Agriculture and Biology 18:975-982.

Blossey, B. and R. Notzold. 1995. Evolution of increased competitive ability in invasive nonindigenous plants: a hypothesis. Journal of Ecology 83:887-889.

Bolmgren, K. and K. Lönnberg. 2005. Herbarium data reveal an association between fleshy fruit type and earlier flowering time. International Journal of Plant Sciences 166:663-670. 
Bone, E. and A. Farres. 2001. Trends and rates of microevolution in plants. Pp. 165-182 in A. P. Hendry, and M. T. Kinnison, eds. Microevolution Rate, Pattern, Process. Springer Netherlands, Dordrecht.

Bradshaw, A. D. 1965. Evolutionary significance of phenotypic plasticity in plants. Pp. 115-155. Advances in Genetics. Elsevier.

Bresson, J., F. Vasseur, M. Dauzat, G. Koch, C. Granier, and D. Vile. 2015. Quantifying spatial heterogeneity of chlorophyll fluorescence during plant growth and in response to water stress. Plant Methods 11:23.

Brock, M. T. and C. Galen. 2005. Drought tolerance in the alpine dandelion, Taraxacum ceratophorum (Asteraceae), its exotic congener T. officinale, and interspecific hybrids under natural and experimental conditions. American Journal of Botany 92:1311-1321.

Browne, R., V. Moller, V. E. Forbes, and M. Depledge. 2002. Estimating genetic and environmental components of variance using sexual and clonal Artemia. Journal of Experimental Marine Biology and Ecology 267:107-119.

Brunet, J. and H. R. Sweet. 2006. Impact of insect pollinator group and floral display size on outcrossing rate. Evolution 60:234-246.

Bunbury-Blanchette, A. L., J. R. Freeland, and M. E. Dorken. 2015. Hybrid typha $\times$ glauca outperforms native T. latifolia under contrasting water depths in a common garden. Basic and applied ecology 16:394-402.

Byers, D. 2008. Components of phenotypic variance. Nature Education 1:161.

Cahaner, A. and J. Hillel. 1980. Estimating heritability and genetic correlation between traits from generations $\mathrm{F} 2$ and $\mathrm{F} 3$ of self-fertilizing species: a comparison of three methods. TAG Theoretical and Applied Genetics 58:33-38.

Campbell, D. R. and N. M. Waser. 2007. Evolutionary dynamics of an Ipomopsis hybrid zone: confronting models with lifetime fitness data. The American Naturalist 169:298-310.

Campbell, D. R. and C. Wendlandt. 2013. Altered precipitation affects plant hybrids differently than their parental species. American journal of botany 100:1322-1331.

Campbell, D. R., C. A. Wu, and S. E. Travers. 2010. Photosynthetic and growth responses of reciprocal hybrids to variation in water and nitrogen availability. American Journal of Botany 97:925-933.

Campbell, L. G., D. Lee, K. Shukla, T. A. Waite, and D. Bartsch. 2016a. An ecological approach to measuring the evolutionary consequences of gene flow from crops to wild or weedy relatives. Applications in Plant Sciences 4:1500114.

Campbell, L. G., R. J. Parker, G. Blakelock, N. Pirimova, and K. L. Mercer. 2015. Maternal environment influences propagule pressure of an invasive plant, Raphanus raphanistrum (Brassicaceae). International Journal of Plant Sciences 176:393-403.

Campbell, L. G., K. Shukla, M. E. Sneck, C. Chaplin, and K. L. Mercer. 2016b. The Effect of Altered Soil Moisture on Hybridization Rate in a Crop-Wild System (Raphanus spp.). PloS One 11:e0166802.

Campbell, L. G. and A. A. Snow. 2007. Competition alters life history and increases the relative fecundity of crop-wild radish hybrids (Raphanus spp.). New Phytologist 173:648-660.

Campbell, L. G. and A. A. Snow. 2009. Can feral weeds evolve from cultivated radish (Raphanus sativus, Brassicaceae)? American Journal of Botany 96:498-506.

Campbell, L. G., A. A. Snow, and C. E. Ridley. 2006. Weed evolution after crop gene introgression: greater survival and fecundity of hybrids in a new environment. Ecology Letters 9:1198-1209. 
Campbell, L. G., A. A. Snow, and P. M. Sweeney. 2009a. When divergent life histories hybridize: insights into adaptive life-history traits in an annual weed. New Phytologist 184:806-818.

Campbell, L. G., A. A. Snow, P. M. Sweeney, and J. M. Ketner. 2009b. Rapid evolution in cropweed hybrids under artificial selection for divergent life histories. Evolutionary Applications 2:172-186.

Campbell, L. G., Z. Teitel, and M. N. Miriti. 2016c. Contemporary evolution and the dynamics of invasion in crop-wild hybrids with heritable variation for two weedy life-histories. Evolutionary Applications.

Campbell, L. G., Z. Teitel, M. N. Miriti, and A. A. Snow. 2014. Context-specific enhanced invasiveness of Raphanus crop-wild hybrids: A test for associations between greater fecundity and population growth. Canadian Journal of Plant Science 94:1315-1324.

Carroll, S. P., H. Dingle, T. R. Famula, and C. W. Fox. 2001. Genetic architecture of adaptive differentiation in evolving host races of the soapberry bug, Jadera haematoloma. Pp. 257-272. Microevolution Rate, Pattern, Process. Springer.

Chapin III, F. S., K. Autumn, and F. Pugnaire. 1993. Evolution of suites of traits in response to environmental stress. The American Naturalist 142:S78-S92.

Chapman, M. A. and J. M. Burke. 2006. Letting the gene out of the bottle: the population genetics of genetically modified crops. New Phytologist 170:429-443.

Charlesworth, D. 2006. Evolution of plant breeding systems. Current Biology 16:R726-R735.

Chaves, M. M., J. P. Maroco, and J. S. Pereira. 2003. Understanding plant responses to drought - from genes to the whole plant. Functional Plant Biology 30:239-264.

Clausen, J., D. Keck, and W. Hiesey. 1948. Experimental studies on the nature of species. III. Environmental responses of climatic races of Achillea. Carnegie Instit. Washington Publ. 581.

Clay, K. 2001. Symbiosis and the regulation of communities. American Zoologist 41:810-824.

Clay, K. and C. Schardl. 2002. Evolutionary origins and ecological consequences of endophyte symbiosis with grasses. The American Naturalist 160:S99-S127.

Clyde, W. C. and P. D. Gingerich. 1994. Rates of evolution in the dentition of early Eocene Cantius: comparison of size and shape. Paleobiology 20:506-522.

Colautti, R. I. and H. J. MacIsaac. 2004. A neutral terminology to define 'invasive'species. Diversity and Distributions 10:135-141.

Conner, J. and S. Via. 1993. Patterns of phenotypic and genetic correlations among morphological and life-history traits in wild radish, Raphanus raphanistrum. Evolution 47:704-711.

Conner, J. K., R. Franks, and C. Stewart. 2003. Expression of additive genetic variances and covariances for wild radish floral traits: comparison between field and greenhouse environments. Evolution 57:487-495.

Conner, J. K. and D. L. Hartl. 2004. A primer of ecological genetics. Sinauer Associates Incorporated.

Conner, J. K., S. Rush, and P. Jennetten. 1996a. Measurements of natural selection on floral traits in wild radish (Raphanus raphanistrum). I. Selection through lifetime female fitness. Evolution 50:1127-1136.

Conner, J. K., S. Rush, S. Kercher, and P. Jennetten. 1996b. Measurements of natural selection on floral traits in wild radish (Raphanus raphanistrum). II. Selection through lifetime male and total fitness. Evolution 50:1137-1146. 
Conover, D. O. and E. T. Schultz. 1995. Phenotypic similarity and the evolutionary significance of countergradient variation. Trends in Ecology \& Evolution 10:248-252.

Crawford, K. and K. Whitney. 2010. Population genetic diversity influences colonization success. Molecular Ecology 19:1253-1263.

Cruzan, M. B. and M. L. Arnold. 1993. Ecological and genetic associations in an Iris hybrid zone. Evolution 47:1432-1445.

Davey, P., A. Parsons, L. Atkinson, K. Wadge, and S. Long. 1999. Does photosynthetic acclimation to elevated $\mathrm{CO} 2$ increase photosynthetic nitrogen-use efficiency? A study of three native UK grassland species in open-top chambers. Functional Ecology 13:21-28.

Davidson, A. M., M. Jennions, and A. B. Nicotra. 2011. Do invasive species show higher phenotypic plasticity than native species and, if so, is it adaptive? A meta-analysis. Ecology Letters 14:419-431.

Debat, V. and P. David. 2001. Mapping phenotypes: canalization, plasticity and developmental stability. Trends in Ecology \& Evolution 16:555-561.

Dechaine, J. M., J. C. Burger, M. A. Chapman, G. J. Seiler, R. Brunick, S. J. Knapp, and J. M. Burke. 2009. Fitness effects and genetic architecture of plant-herbivore interactions in sunflower crop-wild hybrids. New Phytologist 184:828-841.

Dlugosch, K. M. and I. M. Parker. 2008. Invading populations of an ornamental shrub show rapid life history evolution despite genetic bottlenecks. Ecology Letters 11:701-709.

Doebley, J. 1992. Molecular systematics and crop evolution. Pp. 202-222. Molecular systematics of plants. Springer.

Doebley, J. and A. Stec. 1991. Genetic analysis of the morphological differences between maize and teosinte. Genetics 129:285-295.

Donovan, L. A., S. A. Dudley, D. M. Rosenthal, and F. Ludwig. 2007. Phenotypic selection on leaf water use efficiency and related ecophysiological traits for natural populations of desert sunflowers. Oecologia 152:13-25.

Donovan, L. A., H. Maherali, C. M. Caruso, H. Huber, and H. de Kroon. 2011. The evolution of the worldwide leaf economics spectrum. Trends in Ecology \& Evolution 26:88-95.

Dudley, J. 1977. 76 generations of selection for oil and protein percentage in maize. International Conference on Quantitative Genetics. Ames, Iowa (USA). 16-21 Aug 1976.

Dudley, J. and R. Lambert. 1992. Ninety generations of selection for oil and protein in maize. Maydica.

Dudley, S. A. 1996. Differing selection on plant physiological traits in response to environmental water availability: a test of adaptive hypotheses. Evolution 50:92-102.

Eckert, C. G., B. Ozimec, C. R. Herlihy, C. A. Griffin, and M. B. Routley. 2009. Floral morphology mediates temporal variation in the mating system of a self-compatible plant. Ecology 90:1540-1548.

Eizaguirre, C., T. L. Lenz, M. Kalbe, and M. Milinski. 2012. Divergent selection on locally adapted major histocompatibility complex immune genes experimentally proven in the field. Ecology Letters 15:723-731.

Elena, S. F. and R. E. Lenski. 2003. Microbial genetics: evolution experiments with microorganisms: the dynamics and genetic bases of adaptation. Nature Reviews Genetics $4: 457$.

Ellstrand, N. C. 2003. Dangerous liaisons? When cultivated plants mate with their wild relatives. JHU Press. 
Ellstrand, N. C., B. Devlin, and D. L. Marshall. 1989. Gene flow by pollen into small populations: Data from experimental and natural stands of wild radish. Proceedings of the National Academy of Sciences of the United States of America 86:9044-9047.

Ellstrand, N. C., S. M. Heredia, J. A. Leak-Garcia, J. M. Heraty, J. C. Burger, L. Yao, S. Nohzadeh-Malakshah, and C. E. Ridley. 2010. Crops gone wild: evolution of weeds and invasives from domesticated ancestors. Evolutionary Applications 3:494-504.

Ellstrand, N. C. and C. A. Hoffman. 1990. Hybridization as an avenue of escape for engineered genes. BioScience 40:438-442.

Ellstrand, N. C., P. Meirmans, J. Rong, D. Bartsch, A. Ghosh, T. J. de Jong, P. Haccou, B.-R. Lu, A. A. Snow, and C. Neal Stewart Jr. 2013. Introgression of crop alleles into wild or weedy populations. Annual Review of Ecology, Evolution, and Systematics 44:325-345.

Ellstrand, N. C., H. C. Prentice, and J. F. Hancock. 1999. Gene flow and introgression from domesticated plants into their wild relatives. Annual review of Ecology and Systematics 30:539-563.

Ellstrand, N. C. and L. H. Rieseberg. 2016. When gene flow really matters: gene flow in applied evolutionary biology. Evolutionary applications 9:833-836.

Falconer, D. and T. Mackay. 1996. Heritability. Introduction to Quantitative Genetics:160-183.

Falconer, D. S. 1952. The problem of environment and selection. The American Naturalist 86:293-298.

Favaretto, V. F., C. A. Martinez, H. H. Soriani, and R. P. Furriel. 2011. Differential responses of antioxidant enzymes in pioneer and late-successional tropical tree species grown under sun and shade conditions. Environmental and experimental botany 70:20-28.

Fay, P. A., J. D. Carlisle, A. K. Knapp, J. M. Blair, and S. L. Collins. 2000. Altering rainfall timing and quantity in a mesic grassland ecosystem: design and performance of rainfall manipulation shelters. Ecosystems 3:308-319.

Fischer, R. and N. C. Turner. 1978. Plant productivity in the arid and semiarid zones. Annual Review of Plant Physiology 29:277-317.

Flexas, J., J. Bota, F. Loreto, G. Cornic, and T. Sharkey. 2004. Diffusive and metabolic limitations to photosynthesis under drought and salinity in $\mathrm{C} 3$ plants. Plant Biology 6:269-279.

Flint-Garcia, S. A. 2013. Genetics and consequences of crop domestication. Journal of Agricultural and Food Chemistry 61:8267-8276.

Forrest, J., D. W. Inouye, and J. D. Thomson. 2010. Flowering phenology in subalpine meadows: Does climate variation influence community co-flowering patterns? Ecology 91:431-440.

Fox, J. W., S. 2011. An \{R\} Companion to Applied Regression. Sage Publications, Thousand Oaks, California.

Frankham, R. 2005. Genetics and extinction. Biological Conservation 126:131-140.

Franks, S. J. 2011. Plasticity and evolution in drought avoidance and escape in the annual plant Brassica rapa. New Phytologist 190:249-257.

Franks, S. J., S. Sim, and A. E. Weis. 2007. Rapid evolution of flowering time by an annual plant in response to a climate fluctuation. Proceedings of the National Academy of Sciences 104:1278-1282.

Frey, K. and J. Holland. 1999. Nine cycles of recurrent selection for increased groat-oil content in oat. Crop Science 39:1636-1641.

Funk, J. L. 2013. The physiology of invasive plants in low-resource environments. Conservation Physiology 1. 
Gepts, P. 2004. Crop domestication as a long-term selection experiment. Plant breeding reviews 24:1-44.

Germain, R. M., C. M. Caruso, and H. Maherali. 2013. Mechanisms and consequences of water stress-induced parental effects in an invasive annual grass. International Journal of Plant Sciences 174:886-895.

Ghalambor, C. K., J. K. McKay, S. P. Carroll, and D. N. Reznick. 2007. Adaptive versus nonadaptive phenotypic plasticity and the potential for contemporary adaptation in new environments. Functional Ecology 21:394-407.

Gingerich, P. D. 1983. Rates of evolution: effects of time and temporal scaling. Science 222:159161.

Gingerich, P. D. 1993. Quantification and comparison of evolutionary rates. American Journal of Science 293:453-478.

Gingerich, P. D. 2001. Rates of evolution on the time scale of the evolutionary process. Pp. 127144. Microevolution Rate, Pattern, Process. Springer.

Goltsev, V., H. Kalaji, M. Paunov, W. Bąba, T. Horaczek, J. Mojski, H. Kociel, and S. Allakhverdiev. 2016. Variable chlorophyll fluorescence and its use for assessing physiological condition of plant photosynthetic apparatus. Russian journal of plant physiology 63:869-893.

Goodwillie, C., S. Kalisz, and C. G. Eckert. 2005. The Evolutionary Enigma of Mixed Mating Systems in Plants: Occurrence, Theoretical Explanations, and Empirical Evidence. Annual Review of Ecology, Evolution, and Systematics 36:47-79.

Gorné, L. D. and S. Díaz. 2017. A novel meta-analytical approach to improve systematic review of rates and patterns of microevolution. Ecology and Evolution 7:5821-5832.

Gould, J. L. G., C. G. J. L. Gould, and C. G. Gould. 1989. Sexual selection.

Goulet, B. E., F. Roda, and R. Hopkins. 2017. Hybridization in plants: old ideas, new techniques. Plant physiology 173:65-78.

Government of Canada. 2018. Historical Climate Data - Buttonville A in E. a. N. Resources, ed. Government of Canada, Canada.

Gressel, J. 1999. Tandem constructs: preventing the rise of superweeds. Trends in Biotechnology 17:361-366.

Gressel, J. 2005. Crop ferality and volunteerism. CRC Press.

Gressel, J. and H. Al-Ahmad. 2012. Transgenic mitigation of transgene dispersal by pollen and seed. Plant Gene Containment. Ames, IA: Wiley-Blackwell:125-146.

Haldane, J. B. S. 1949. Suggestions as to quantitative measurement of rates of evolution. Evolution:51-56.

Halpin, P. N. 1997. Global climate change and natural area protection: Management responses and research directions Ecological Applications 7:828-843.

Han, Q., H. Higashi, Y. Mitsui, and H. Setoguchi. 2016. Lineage isolation in the face of active gene flow in the coastal plant wild radish is reinforced by differentiated vernalisation responses. BMC Evolutionary Biology 16:84.

Hancock, J. F. 2012. Plant evolution and the origin of crop species. CABI.

Hard, J. J., W. E. Bradshaw, and C. M. Holzapfel. 1993. Genetic coordination of demography and phenology in the pitcher-plant mosquito, Wyeomyia smithii. Journal of Evolutionary Biology 6:707-723.

Harlan, J. R. 1992. Crops and man. American Society of Agronomy.

Hartl, D. L. and A. Clark. 2007. Principles of Population Genetics (Sinauer, Sunderland, MA). 
Haugen, T. O. and L. A. Vøllestad. 2001. A century of life-history evolution in grayling. Genetica 112:475-491.

Heap, I. 2018. The International survey of herbicide resistant weeds. Online. Internet.

Hegde, S. G., J. D. Nason, J. M. Clegg, and N. C. Ellstrand. 2006. The evolution of California's wild radish has resulted in the extinction of its progenitors. Evolution 60:1187-1197.

Hendry, A. P., T. J. Farrugia, and M. T. Kinnison. 2008. Human influences on rates of phenotypic change in wild animal populations. Molecular Ecology 17:20-29.

Hendry, A. P. and M. T. Kinnison. 1999. Perspective: the pace of modern life: measuring rates of contemporary microevolution. Evolution 53:1637-1653.

Heredia, S. M. and N. C. Ellstrand. 2014. Novel seed protection in the recently evolved invasive, California wild radish, a hybrid Raphanus sp.(Brassicaceae). American Journal of Botany 101:2043-2051.

Hoffmann, A. A. and C. M. Sgrò. 2011. Climate change and evolutionary adaptation. Nature 470:479.

Holm, L. 1997. World weeds: natural histories and distribution. John Wiley \& Sons.

Hooftman, D. A., J. G. B. Oostermeijer, M. M. Jacobs, and H. C. Den Nijs. 2005. Demographic vital rates determine the performance advantage of crop-wild hybrids in lettuce. Journal of Applied Ecology 42:1086-1095.

Hovick, S. M., L. G. Campbell, A. A. Snow, and K. D. Whitney. 2012. Hybridization alters early life-history traits and increases plant colonization success in a novel region. The American Naturalist 179:192-203.

Hovick, S. M. and K. D. Whitney. 2014. Hybridisation is associated with increased fecundity and size in invasive taxa: meta-analytic support for the hybridisation-invasion hypothesis. Ecology letters 17:1464-1477.

Irwin, Rebecca E. and Sharon Y. Strauss. 2005. Flower color microevolution in wild radish: Evolutionary response to pollinator-mediated election. The American Naturalist 165:225237.

Irwin, R. E., S. Y. Strauss, S. Storz, A. Emerson, and G. Guibert. 2003. The role of herbivores in the maintenance of a flower colour polymorphism in wild radish. Ecology 84:1733-1743.

Jenczewski, E., J. Ronfort, and A.-M. Chèvre. 2003. Crop-to-wild gene flow, introgression and possible fitness effects of transgenes. Environmental Biosafety Research 2:9-24.

Johnson, W., L. Jackson, O. Ochoa, R. Van Wijk, J. Peleman, D. S. Clair, and R. Michelmore. 2000. Lettuce, a shallow-rooted crop, and Lactuca serriola, its wild progenitor, differ at QTL determining root architecture and deep soil water exploitation. Theoret. Appl. Genetics 101:1066-1073.

Jones, N., B. Husband, and A. MacDougall. 2013. Reproductive system of a mixed-mating plant responds to climate perturbation by increased selfing. Proceedings of the Royal Society of London B: Biological Sciences 280:20131336.

Jørgensen, R., B. Andersen, T. P. Hauser, L. Landbo, T. R. Mikkelsen, and H. Østergård. 1997. Introgression of crop genes from oilseed rape (Brassica napus) to related wild species-an avenue for the escape of engineered genes. Pp. 211-218. International Symposium Brassica 97, Xth Crucifer Genetics Workshop 459.

Kannadan, S. and J. Rudgers. 2008. Endophyte symbiosis benefits a rare grass under low water availability. Functional Ecology 22:706-713.

Kareiva, P., W. Morris, and C. M. Jacobi. 1994. Studying and managing the risk of crossfertilization between transgenic crops and wild relatives. Molecular Ecology 3:15-21. 
Kay, Q. 1976. Preferential pollination of yellow-flowered morphs of Raphanus raphanistrum by Pieris and Eristalis spp. Nature 261:230.

Keller, S. and D. Taylor. 2010. Genomic admixture increases fitness during a biological invasion. Journal of evolutionary biology 23:1720-1731.

Kingsolver, J. G., H. E. Hoekstra, J. M. Hoekstra, D. Berrigan, S. N. Vignieri, C. Hill, A. Hoang, P. Gibert, and P. Beerli. 2001. The strength of phenotypic selection in natural populations. The American Naturalist 157:245-261.

Kinnison, M. T. and N. G. Hairston. 2007. Eco-evolutionary conservation biology: contemporary evolution and the dynamics of persistence. Functional Ecology 21:444-454.

Klier, K., M. Leoschke, and J. Wendel. 1991. Hybridization and introgression in white and yellow ladyslipper orchids (Cypripedium candidum and $C$. pubescens). Journal of Heredity 82:305-318.

Klinger, T., D. R. Elam, and N. C. Ellstrand. 1991. Radish as a model system for the study of engineered gene escape rates via crop-weed mating. Conservation Biology 5:531-535.

Kooyers, N. J. 2015. The evolution of drought escape and avoidance in natural herbaceous populations. Plant Science 234:155-162.

Kost, M. A., H. M. Alexander, D. Jason Emry, and K. L. Mercer. 2015. Life history traits and phenotypic selection among sunflower crop-wild hybrids and their wild counterpart: implications for crop allele introgression. Evolutionary applications 8:510-524.

Kriebel, D., J. Tickner, P. Epstein, J. Lemons, R. Levins, E. L. Loechler, M. Quinn, R. Rudel, T. Schettler, and M. Stoto. 2001. The precautionary principle in environmental science. Environmental Health Perspectives 109:871-876.

Ladizinsky, G. 1985. Founder effect in crop-plant evolution. Economic Botany 39:191-199.

Lambert, R., D. Alexander, E. Mollring, and B. Wiggens. 1997. Selection for increased oil concentration in maize kernels and associated changes in several kernel traits. Maydica 42:39-43.

Lande, R. and S. J. Arnold. 1983. The measurement of selection on correlated characters Evolution 37:1210-1226.

Lande, R. and S. Shannon. 1996. The role of genetic variation in adaptation and population persistence in a changing environment. Evolution 50:434-437.

Lanfear, R., H. Kokko, and A. Eyre-Walker. 2014. Population size and the rate of evolution. Trends in Ecology \& Evolution 29:33-41.

Langevin, S. A., K. Clay, and J. B. Grace. 1990. The incidence and effects of hybridization between cultivated rice and its related weed red rice (Oryza sativa L.). Evolution 44:1000-1008.

Larios, E. and D. L. Venable. 2015. Maternal adjustment of offspring provisioning and the consequences for dispersal. Ecology 96:2771-2780.

Lee, C. E. 2002. Evolutionary genetics of invasive species. Trends in ecology \& evolution 17:386-391.

Leger, E. A. and E. K. Espeland. 2010. Coevolution between native and invasive plant competitors: implications for invasive species management. Evolutionary applications 3:169-178.

Lemons, A., K. Clay, and J. A. Rudgers. 2005. Connecting plant-microbial interactions above and belowground: a fungal endophyte affects decomposition. Oecologia 145:595-604. 
Lenski, R. E., M. R. Rose, S. C. Simpson, and S. C. Tadler. 1991. Long-term experimental evolution in Escherichia coli. I. Adaptation and divergence during 2,000 generations. The American Naturalist 138:1315-1341.

Levin, D. A. 2010. Environment-enhanced self-fertilization: implications for niche shifts in adjacent populations. Journal of Ecology 98:1276-1283.

Levitt, J. 1980. Responses of Plants to Environmental Stress, Volume 1: Chilling, Freezing, and High Temperature Stresses. Academic Press.

Lewis-Jones, L., J. Thorpe, and G. Wallis. 1982. Genetic divergence in four species of the genus Raphanus: implications for the ancestry of the domestic radish $R$. sativus. Biological Journal of the Linnean Society 18:35-48.

Linder, C., I. Taha, L. Rieseberg, G. Seiler, and A. Snow. 1998. Long-term introgression of crop genes into wild sunflower populations. Theoret. Appl. Genetics 96:339-347.

Liu, F., H. Zhao, L. Yao, J. Zhang, and M. Cao. 2003. Gene flow from transgenic chinese cabbage (Brassica campestris ssp chinensis var. utilis) to related Cruciferae species through outcrossing. Acta Botanica Sinica 45:681-687.

Liu, Y.-B., H. Darmency, C. N. Stewart, W. Wei, Z.-X. Tang, and K.-P. Ma. 2015a. The effect of Bt-transgene introgression on plant growth and reproduction in wild Brassica juncea. Transgenic Research 24:537-547.

Liu, Y.-X., W.-P. Zhang, J.-H. Sun, X.-F. Li, P. Christie, and L. Li. 2015b. High morphological and physiological plasticity of wheat roots is conducive to higher competitive ability of wheat than maize in intercropping systems. Plant and Soil 397:387-399.

Lodge, D. M., P. W. Simonin, S. W. Burgiel, R. P. Keller, J. M. Bossenbroek, C. L. Jerde, A. M. Kramer, E. S. Rutherford, M. A. Barnes, and M. E. Wittmann. 2016. Risk analysis and bioeconomics of invasive species to inform policy and management. Annual Review of Environment and Resources 41.

Losos, J. B., K. I. Warheitt, and T. W. Schoener. 1997. Adaptive differentiation following experimental island colonization in Anolis lizards. Nature 387:70.

Ludwig, F., D. M. Rosenthal, J. A. Johnston, N. Kane, B. L. Gross, C. Lexer, S. A. Dudley, L. H. Rieseberg, and L. A. Donovan. 2004. Selection on leaf ecophysiological traits in a desert hybrid Helianthus species and early-generation hybrids. Evolution 58:2682-2692.

Luke, S. G. 2017. Evaluating significance in linear mixed-effects models in R. Behavior Research Methods 49:1494-1502.

Ma, F., C. Zhao, R. Milne, M. Ji, L. Chen, and J. Liu. 2010. Enhanced drought-tolerance in the homoploid hybrid species Pinus densata: implication for its habitat divergence from two progenitors. New Phytologist 185:204-216.

Mallet, J. 2007. Hybrid speciation. Nature 446:279-283.

Maron, J. L., M. Vilà, R. Bommarco, S. Elmendorf, and P. Beardsley. 2004. Rapid evolution of an invasive plant. Ecological Monographs 74:261-280.

Marshall, L. G. and R. S. Corruccini. 1978. Variability, evolutionary rates, and allometry in dwarfing lineages. Paleobiology 4:101-119.

Martin, N. H., A. C. Bouck, and M. L. Arnold. 2006. Detecting Adaptive Trait Introgression between Iris fulva and Iris brevicaulis in highly-selective field conditions. Genetics.

Mathys, B. A. and J. L. Lockwood. 2009. Rapid evolution of great kiskadees on Bermuda: an assessment of the ability of the island rule to predict the direction of contemporary evolution in exotic vertebrates. Journal of Biogeography 36:2204-2211. 
Maxwell, K. and G. N. Johnson. 2000. Chlorophyll fluorescence - a practical guide. Journal of Experimental Botany 51:659-668.

Mercer, K. L., D. A. Andow, D. L. Wyse, and R. G. Shaw. 2007. Stress and domestication traits increase the relative fitness of crop-wild hybrids in sunflower. Ecology letters 10:383393.

Mercer, K. L., D. J. Emry, A. A. Snow, M. A. Kost, B. A. Pace, and H. M. Alexander. 2014. Fitness of crop-wild hybrid sunflower under competitive conditions: implications for crop-to-wild introgression. PloS one 9:e109001.

Merilä, J., B. Sheldon, and L. Kruuk. 2001. Explaining stasis: microevolutionary studies in natural populations. Genetica 112:199-222.

Metz, J., J. von Oppen, and K. Tielbörger. 2015. Parental environmental effects due to contrasting watering adapt competitive ability, but not drought tolerance, in offspring of a semi-arid annual Brassicaceae. Journal of Ecology 103:990-997.

Michaletz, S. T., D. Cheng, A. J. Kerkhoff, and B. J. Enquist. 2014. Convergence of terrestrial plant production across global climate gradients. Nature 512:39.

Mitchell-Olds, T. and J. Rutledge. 1986. Quantitative genetics in natural plant populations: a review of the theory. The American Naturalist 127:379-402.

Moczek, A. P., S. Sultan, S. Foster, C. Ledón-Rettig, I. Dworkin, H. F. Nijhout, E. Abouheif, and D. W. Pfennig. 2011. The role of developmental plasticity in evolutionary innovation. Proceedings of the Royal Society of London B: Biological Sciences 278:2705-2713.

Mooney, H. A. and S. L. Gulmon. 1979. Environmental and Evolutionary Constraints on the Photosynthetic Characteristics of Higher Plants. Pp. 316-337 in O. T. Solbrig, S. Jain, G. B. Johnson, and P. H. Raven, eds. Topics in Plant Population Biology. Macmillan Education UK, London.

Moran, E. V. and J. M. Alexander. 2014. Evolutionary responses to global change: lessons from invasive species. Ecology Letters 17:637-649.

Mutegi, E., A. A. Snow, M. Rajkumar, R. Pasquet, H. Ponniah, M.-C. Daunay, and P. Davidar. 2015. Genetic diversity and population structure of wild/weedy eggplant (Solanum insanum, Solanaceae) in southern India: Implications for conservation. American Journal of Botany 102:140-148.

Nicotra, A. B., O. K. Atkin, S. P. Bonser, A. M. Davidson, E. Finnegan, U. Mathesius, P. Poot, M. D. Purugganan, C. Richards, and F. Valladares. 2010. Plant phenotypic plasticity in a changing climate. Trends in Plant Science 15:684-692.

Nosil, P., D. J. Funk, and D. Ortiz-Barrientos. 2009. Divergent selection and heterogeneous genomic divergence. Molecular Ecology 18:375-402.

Ocampo, J. A. 1980. Effect of crop rotations involving host and non-host plants on vesiculararbuscular mycorrhizal infection of host plants. Plant and Soil 56:283-291.

Ohashi, Y., N. Nakayama, H. Saneoka, and K. Fujita. 2006. Effects of Drought Stress on Photosynthetic Gas Exchange, Chlorophyll Fluorescence and Stem Diameter of Soybean Plants. Biologia Plantarum 50:138-141.

Pandolfo, C. E., A. Presotto, F. Moreno, I. Dossou, J. P. Migasso, E. Sakima, and M. Cantamutto. 2016. Broad resistance to acetohydroxyacid-synthase-inhibiting herbicides in feral radish (Raphanus sativus L.) populations from Argentina. Pest Management Science 72:354-361.

Panetsos, C. and H. Baker. 1967. The origin of variation in "wild" Raphanus sativus (Cruciferae) in California. Genetica 38:243-274. 
Papa, R. and P. Gepts. 2003. Asymmetry of gene flow and differential geographical structure of molecular diversity in wild and domesticated common bean (Phaseolus vulgaris L.) from Mesoamerica. Theoret. Appl. Genetics 106:239-250.

Parker, P. G., A. A. Snow, M. D. Schug, G. C. Booton, and P. A. Fuerst. 1998. What molecules can tell us about populations: choosing andusing a molecular marker. Ecology 79:361382.

Parmesan, C. and G. Yohe. 2003. A globally coherent fingerprint of climate change impacts across natural systems. Nature 421:37-42.

Picard, C. and M. Bosco. 2005. Maize heterosis affects the structure and dynamics of indigenous rhizospheric auxins-producing Pseudomonas populations. FEMS Microbiology Ecology 53:349-357.

Pirimova, N., A. J. Parker, and L. G. Campbell. 2015. Does altering local water availability for an invasive plant (Raphanus raphanistrum) affect floral morphology and reproductive potential. Am J Undergrad Res 12:63-72.

Pleijel, H. and P. Högy. 2015. CO2 dose-response functions for wheat grain, protein and mineral yield based on FACE and open-top chamber experiments. Environmental pollution 198:70-77.

Presotto, A., C. Pandolfo, M. Poverene, and M. Cantamutto. 2016. Can achene selection in sunflower crop-wild hybrids by pre-dispersal seed predators hasten the return to phenotypically wild sunflowers? Euphytica 208:453-462.

Purugganan, M. D. and D. Q. Fuller. 2011. Archaeological data reveal slow rates of evolution during plant domestication. Evolution 65:171-183.

Rabus, M., T. Söllradl, H. Clausen-Schaumann, and C. Laforsch. 2013. Uncovering ultrastructural defences in Daphnia magna-an interdisciplinary approach to assess the predator-induced fortification of the carapace. PloS One 8:e67856.

Rauf, S. 2008. Breeding sunflower (Helianthus annuus L.) for drought tolerance. Communications in Biometry and Crop Science 3:29-44.

Reeves, T., G. Code, and C. Piggin. 1981. Seed production and longevity, seasonal emergence and phenology of wild radish (Raphanus raphanistrum L.). Australian Journal of Experimental Agriculture 21:524-530.

Reichman, J. R., L. S. Watrud, E. H. Lee, C. A. Burdick, M. A. Bollman, M. J. Storm, G. A. King, and C. Mallory-Smith. 2006. Establishment of transgenic herbicide-resistant creeping bentgrass (Agrostis stolonifera L.) in nonagronomic habitats. Molecular Ecology 15:4243-4255.

Reznick, D. N., F. H. Shaw, F. H. Rodd, and R. G. Shaw. 1997. Evaluation of the rate of evolution in natural populations of guppies (Poecilia reticulata). Science 275:1934-1937.

Rhymer, J. M. and D. Simberloff. 1996. Extinction by hybridization and introgression Annual Review of Ecology and Systematics 27:83-109.

Richards, C. L., O. Bossdorf, N. Z. Muth, J. Gurevitch, and M. Pigliucci. 2006. Jack of all trades, master of some? On the role of phenotypic plasticity in plant invasions. Ecology Letters 9:981-993.

Richardson, D. M., P. Pyšek, M. Rejmánek, M. G. Barbour, F. D. Panetta, and C. J. West. 2000. Naturalization and invasion of alien plants: concepts and definitions. Diversity and Distributions 6:93-107.

Ridley, C. E. and L. C. Alexander. 2016. Applying gene flow science to environmental policy needs: a boundary work perspective. Evolutionary applications 9:924-936. 
Ridley, C. E. and N. C. Ellstrand. 2008. Evolution of enhanced reproduction in the hybridderived invasive, California wild radish (Raphanus sativus). Biological Invasions $11: 2251$.

Ridley, C. E. and N. C. Ellstrand. 2010. Rapid evolution of morphology and adaptive life history in the invasive California wild radish (Raphanus sativus) and the implications for management. Evolutionary Applications 3:64-76.

Rieseberg, L. H., M. A. Archer, and R. K. Wayne. 1999. Transgressive segregation, adaptation and speciation. Heredity 83:363.

Rieseberg, L. H. and S. E. Carney. 1998. Plant hybridization. The New Phytologist 140:599-624.

Rieseberg, L. H., O. Raymond, D. M. Rosenthal, Z. Lai, K. Livingstone, T. Nakazato, J. L. Durphy, A. E. Schwarzbach, L. A. Donovan, and C. Lexer. 2003. Major ecological transitions in wild sunflowers facilitated by hybridization. Science 301:1211-1216.

Rose, C. W., R. J. Millwood, H. S. Moon, M. R. Rao, M. D. Halfhill, P. L. Raymer, S. I. Warwick, H. Al-Ahmad, J. Gressel, and C. N. Stewart. 2009. Genetic load and transgenic mitigating genes in transgenic Brassica rapa (field mustard) $\times$ Brassica napus (oilseed rape) hybrid populations. BMC Biotechnology 9:93.

Rubio-Meléndez, M. E., D. A. Sepúlveda, and C. C. Ramírez. 2018. Temporal and spatial distribution of insecticide-resistance mutations in the green peach aphid Myzus persicae (Hemiptera: Aphididae) on primary and secondary host plants in central Chile. Pest Management Science 74:340-347.

Saccardy, K., B. Pineau, O. Roche, and G. Cornic. 1998. Photochemical efficiency of photosystem II and xanthophyll cycle components in Zea mays leaves exposed to water stress and high light. Photosynthesis Research 56:57-66.

Sadras, V., M. Reynolds, A. De la Vega, P. Petrie, and R. Robinson. 2009. Phenotypic plasticity of yield and phenology in wheat, sunflower and grapevine. Field Crops Research 110:242-250.

Sahli, H., J. K. Conner, F. H. Shaw, S. Howe, and A. Lale. 2008. Adaptive differentiation of quantitative traits in the globally distributed weed, wild radish (Raphanus raphanistrum). Genetics.

Sakai, A. K., F. W. Allendorf, J. S. Holt, D. M. Lodge, J. Molofsky, K. A. With, S. Baughman, R. J. Cabin, J. E. Cohen, and N. C. Ellstrand. 2001. The population biology of invasive species. Annual Review of Ecology and Systematics:305-332.

Santos, E. S. A., I. G. Jamieson, L. L. S. Santos, and S. Nakagawa. 2013. Low genetic and morphological differentiation between an introduced population of dunnocks in New Zealand and an ancestral population in England. Biological Invasions 15:185-197.

Sapir, Y., K. Karoly, V. A. Koelling, H. F. Sahli, F. N. Knapczyk, and J. K. Conner. 2017. Effect of expanded variation in anther position on pollinator visitation to wild radish, Raphanus raphanistrum. Annals of botany 120:665-672.

Sargent, R. and B. Roitberg. 2000. Seasonal decline in male-phase duration in a protandrous plant: a response to increased mating opportunities? Functional Ecology 14:484-489.

Saxena, D., S. Flores, and G. Stotzky. 2002. Bt toxin is released in root exudates from 12 transgenic corn hybrids representing three transformation events. Soil Biology and Biochemistry 34:133-137.

Sayed, O. 2003. Chlorophyll Fluorescence as a Tool in Cereal Crop Research. Photosynthetica 41:321-330. 
Scheffler, J. A. and P. J. Dale. 1994. Opportunities for gene transfer from transgenic oilseed rape (Brassica napus) to related species. Transgenic research 3:263-278.

Schierenbeck, K. A. and N. C. Ellstrand. 2009. Hybridization and the evolution of invasiveness in plants and other organisms. Biological invasions 11:1093-1105.

Sharpe, D. M., K. Räsänen, D. Berner, and A. P. Hendry. 2008. Genetic and environmental contributions to the morphology of lake and stream stickleback: implications for gene flow and reproductive isolation. Evolutionary Ecology Research 10:849-866.

Sherrard, M. E. and H. Maherali. 2006. The adaptive significance of drought escape in Avena barbata, an annual grass. Evolution 60:2478-2489.

Sherrard, M. E., H. Maherali, and R. G. Latta. 2009. Water stress alters the genetic architecture of functional traits associated with drought adaptation in Avena barbata. Evolution: International Journal of Organic Evolution 63:702-715.

Shisanya, C. A. 2002. Improvement of drought adapted tepary bean (Phaseolusacutifolius A. Gray var. latifolius) yield through biological nitrogen fixation in semi-arid SE-Kenya. European Journal of Agronomy 16:13-24.

Slatkin, M. 1987. Gene flow and the geographic structure of natural populations. Science 236:787-792.

Sneck, M. 2012. Evolutionary responses to global change: an experimental test of the effect of altered precipitation on hybridization rates in sunflower (Helianthus). Rice University.

Snow, A., T. Culley, L. Campbell, P. Sweeney, S. Hegde, and N. Ellstrand. 2010. Long-term persistence of crop alleles in weedy populations of wild radish (Raphanus raphanistrum). New Phytologist 186:537-548.

Snow, A., P. Moran-Palma, L. Rieseberg, A. Wszelaki, and G. Seiler. 1998. Fecundity, phenology, and seed dormancy of F1 wild-crop hybrids in Sunflower (Helianthus annuus, Asteraceae). American Journal of Botany 85:794-794.

Snow, A. A. 2002. Transgenic crops - why gene flow matters. Nature biotechnology 20:542542.

Snow, A. A., B. Andersen, and R. B. Jørgensen. 1999. Costs of transgenic herbicide resistance introgressed from Brassica napus into weedy B. rapa. Molecular Ecology 8:605-615.

Snow, A. A. and L. G. Campbell. 2005. Can feral radishes become weeds. Crop Ferality and Volunteerism:193-208.

Snow, A. A. and P. M. Palma. 1997. Commercialization of transgenic plants: potential ecological risks. BioScience 47:86-96.

Snow, A. A., D. Pilson, L. H. Rieseberg, M. J. Paulsen, N. Pleskac, M. R. Reagon, D. E. Wolf, and S. M. Selbo. 2003. A Bt transgene reduced herbivory and enhanced fecundity in wild sunflowers. Ecological Applications 13:279-286.

Snow, A. A., K. L. Uthus, and T. M. Culley. 2001. Fitness of hybrids between weedy and cultivated radish: implications for weed evolution. Ecological Applications 11:934-943.

Sørensen, B. S., L. P. Kiær, R. B. Jørgensen, and T. P. Hauser. 2007. The temporal development in a hybridizing population of wild and cultivated chicory (Cichorium intybus L.). Molecular Ecology 16:3292-3298.

Stanton, M. L. 1987. Reproductive biology of petal color variants in wild populations of Raphanus sativus: I. Pollinator response to color morphs. American Journal of Botany 74:178-187. 
Stanton, M. L., A. A. Snow, S. N. Handel, and J. Bereczky. 1989. The impact of a flower-color polymorphism on mating patterns in experimental populations of wild radish (Raphanus raphanistrum L.). Evolution 43:335-346.

Stewart, C., J. All, P. Raymer, and S. Ramachandran. 1997. Increased fitness of transgenic insecticidal rapeseed under insect selection pressure. Molecular Ecology 6:773-779.

Stewart Jr, C. N., M. D. Halfhill, and S. I. Warwick. 2003. Genetic modification: transgene introgression from genetically modified crops to their wild relatives. Nature Reviews Genetics 4:806.

Strand, J. A. and S. E. B. Weisner. 2004. Phenotypic plasticity - contrasting species-specific traits induced by identical environmental constraints. New Phytologist 163:449-451.

Strauss, S. Y., R. E. Irwin, and V. M. Lambrix. 2004. Optimal defence theory and flower petal colour predict variation in the secondary chemistry of wild radish. Journal of Ecology 92:132-141.

Sultan, S. 1995. Phenotypic plasticity and plant adaptation. Acta Botanica Neerlandica 44:363383.

Sultan, S. E. 2000. Phenotypic plasticity for plant development, function and life history. Trends in Plant Science 5:537-542.

Tang, S., R. Okashah, S. Knapp, M. Arnold, and N. Martin. 2010. Reproductive isolation in Louisiana irises: transmission ratio distortion. BMC Plant Biol 10:48.

Taxak, A. K., A. Murumkar, and D. Arya. 2014. Long term spatial and temporal rainfall trends and homogeneity analysis in Wainganga basin, Central India. Weather and Climate Extremes 4:50-61.

Taylor, S. J., M. Arnold, and N. H. Martin. 2009. The genetic architecture of reproductive isolation in Louisana Irises: Hybrid fitness in nature. Evolution 63:2581-2594.

Team, R. C. 2017. R: A language and environment for statistical computing. R Foundation for Statistical Computing, Vienna, Austria.

Teitel, Z., A. Klimowski, and L. G. Campbell. 2016a. Assessing the effects of hybridization and precipitation on invasive weed demography using strength of selection on vital rates. BMC Evolutionary Biology 16:266.

Teitel, Z., A. Laursen, and L. Campbell. 2016b. Germination rates of weedy radish populations (Raphanus spp.) altered by crop-wild hybridisation, not human-mediated changes to soil moisture. Weed Research.

Teitel, Z., A. E. Laursen, and L. G. Campbell. 2016c. Germination rates of weedy radish populations (Raphanus spp.) altered by crop-wild hybridisation, not human-mediated changes to soil moisture. Weed Research 56:149-158.

Tesso, T., I. Kapran, C. Grenier, A. Snow, P. Sweeney, J. Pedersen, D. Marx, G. Bothma, and G. Ejeta. 2008. The Potential for Crop-to-Wild Gene Flow in Sorghum in Ethiopia and Niger: A Geographic Survey Crop Science 48:1425-1431.

Thorpe, R. S., J. T. Reardon, and A. Malhotra. 2005. Common garden and natural selection experiments support ecotypic differentiation in the Dominican anole (Anolis oculatus). The American Naturalist 165:495-504.

Todesco, M., M. A. Pascual, G. L. Owens, K. L. Ostevik, B. T. Moyers, S. Hübner, S. M. Heredia, M. A. Hahn, C. Caseys, and D. G. Bock. 2016. Hybridization and extinction. Evolutionary Applications 9:892-908. 
Turcotte, M. M., N. E. Turley, and M. T. J. Johnson. 2014. The impact of domestication on resistance to two generalist herbivores across 29 independent domestication events. New Phytologist 204:671-681.

Underground, W. Pp. Weather Data. TWC Product and Technology.

Vacher, C., A. E. Weis, D. Hermann, T. Kossler, C. Young, and M. E. Hochberg. 2004. Impact of ecological factors on the initial invasion of $\mathrm{Bt}$ transgenes into wild populations of birdseed rape (Brassica rapa). Theoret. Appl. Genetics 109:806-814.

Van Etten, M. L. and J. Brunet. 2013. The impact of global warming on floral traits that affect the selfing rate in a high-altitude plant. International Journal of Plant Sciences 174:10991108.

Via, S., R. Gomulkiewicz, G. De Jong, S. M. Scheiner, C. D. Schlichting, and P. H. Van Tienderen. 1995. Adaptive phenotypic plasticity: consensus and controversy. Trends in Ecology \& Evolution 10:212-217.

Via, S. and R. Lande. 1985. Genotype-environment interaction and the evolution of phenotypic plasticity. Evolution 39:505-522.

Vilà, M., E. Weber, and C. M. D. Antonio. 2000. Conservation implications of invasion by plant hybridization. Biological Invasions 2:207-217.

Vilà, M., E. Weber, and C. M. D'Antonio. 1998. Flowering and mating system in hybridizing Carpobrotus (Aizoaceae) in coastal California. canadian Journal of Botany 76:11651169.

Vitasse, Y., S. Delzon, C. C. Bresson, R. Michalet, and A. Kremer. 2009. Altitudinal differentiation in growth and phenology among populations of temperate-zone tree species growing in a common garden. Canadian Journal of Forest Research 39:12591269.

Wadgymar, S. M., M. N. Cumming, and A. E. Weis. 2015. The success of assisted colonization and assisted gene flow depends on phenology. Global change biology 21:3786-3799.

Walter, J., D. E. Harter, C. Beierkuhnlein, and A. Jentsch. 2016. Transgenerational effects of extreme weather: perennial plant offspring show modified germination, growth and stoichiometry. Journal of Ecology 104:1032-1040.

Warschefsky, E., R. V. Penmetsa, D. R. Cook, and E. J. von Wettberg. 2014. Back to the wilds: tapping evolutionary adaptations for resilient crops through systematic hybridization with crop wild relatives. American Journal of Botany 101:1791-1800.

Warwick, S. I., H. J. Beckie, and L. M. Hall. 2009. Gene flow, invasiveness, and ecological impact of genetically modified crops. Annals of the New York Academy of Sciences 1168:72-99.

Warwick, S. I. and C. Stewart. 2005. Crops come from wild plants: how domestication, transgenes, and linkage together shape ferality. Crop ferality and volunteerism 36:9-30.

Watrud, L. S., E. H. Lee, A. Fairbrother, C. Burdick, J. R. Reichman, M. Bollman, M. Storm, G. King, and P. K. Van de Water. 2004. Evidence for landscape-level, pollen-mediated gene flow from genetically modified creeping bentgrass with CP4 EPSPS as a marker. Proceedings of the National Academy of Sciences of the United States of America 101:14533-14538.

Weiner, J., L. G. Campbell, J. Pino, and L. Echarte. 2009. The allometry of reproduction within plant populations. Journal of Ecology 97:1220-1233.

Wells, M. S. and D. G. Lloyd. 1991. Dichogamy, gender variation and bet-hedging in Pseudowintera colorata. Evolutionary Ecology 5:310-326. 
Whitney, K. D., J. R. Ahern, and L. G. Campbell. 2009. Hybridization-prone plant families do not generate more invasive species. Biological Invasions 11:1205-1215.

Whitney, K. D., J. R. Ahern, L. G. Campbell, L. P. Albert, and M. S. King. 2010. Patterns of hybridization in plants. Perspectives in Plant Ecology, Evolution and Systematics 12:175182.

Whitney, K. D., K. W. Broman, N. C. Kane, S. M. Hovick, R. A. Randell, and L. H. Rieseberg. 2015. Quantitative trait locus mapping identifies candidate alleles involved in adaptive introgression and range expansion in a wild sunflower. Molecular ecology 24:2194-2211.

Whitney, K. D. and E. Gering. 2015. Five decades of invasion genetics. The New phytologist 205:472.

Whitney, Kenneth D., Rebecca A. Randell, and Loren H. Rieseberg. 2006. Adaptive Introgression of Herbivore Resistance Traits in the Weedy Sunflower Helianthus annuus. The American Naturalist 167:794-807.

Whitton, J., D. Wolf, D. Arias, A. Snow, and L. Rieseberg. 1997. The persistence of cultivar alleles in wild populations of sunflowers five generations after hybridization. Theoret. Appl. Genetics 95:33-40.

Wiersma, A. T., T. A. Gaines, C. Preston, J. P. Hamilton, D. Giacomini, C. R. Buell, J. E. Leach, and P. Westra. 2015. Gene amplification of 5-enol-pyruvylshikimate-3-phosphate synthase in glyphosate-resistant Kochia scoparia. Planta 241:463-474.

Williams, D. G., R. N. Mack, and R. A. Black. 1995. Ecophysiology of introduced Pennisetum setaceum on Hawaii: the role of phenotypic plasticity. Ecology 76:1569-1580.

Wolf, J. B. and M. J. Wade. 2009. What are maternal effects (and what are they not)? Philosophical Transactions of the Royal Society B: Biological Sciences 364:1107-1115.

Wright, S. 1968. Evolution and the genetics of populations. Vol. 1. Genetic and biométrie foundations. London and Chicago: University of Chicago Press.

Wu, C. A. and D. R. Campbell. 2006. Environmental stressors differentially affect leaf ecophysiological responses in two Ipomopsis species and their hybrids. Oecologia 148:202.

Xie, J.-B., G.-Q. Xu, G. D. Jenerette, Y.-f. Bai, Z.-Y. Wang, and Y. Li. 2015. Apparent plasticity in functional traits determining competitive ability and spatial distribution: a case from desert. Scientific Reports 5:12174.

Yahdjian, L. and O. E. Sala. 2002. A rainout shelter design for intercepting different amounts of rainfall. Oecologia 133:95-101.

Yamagishi, H. and T. Terachi. 2003. Multiple origins of cultivated radishes as evidenced by a comparison of the structural variations in mitochondrial DNA of Raphanus. Genome 46:89-94.

Yamane, K., N. Lü, and O. Ohnishi. 2005. Chloroplast DNA variations of cultivated radish and its wild relatives. Plant Science 168:627-634.

Younginger, B. S., D. Sirová, M. B. Cruzan, and D. J. Ballhorn. 2017. Is biomass a reliable estimate of plant fitness? Applications in Plant Sciences 5:1600094.

Zar, J. 1999. Biostatistical analysis. Prentice Hall, Upper Saddle River, New Jersey. 